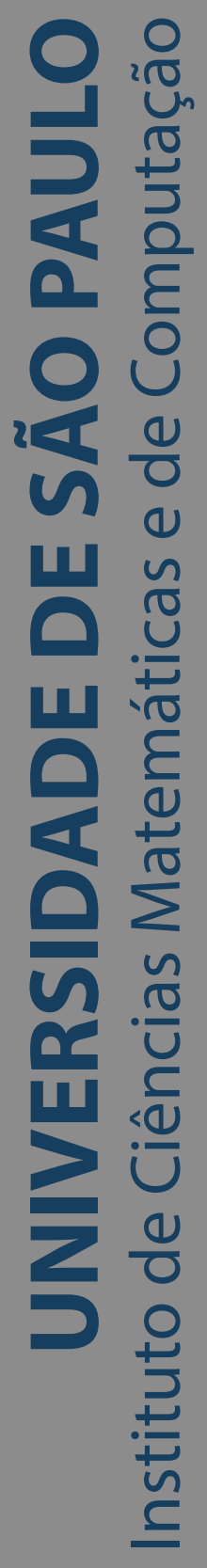

\title{
Topological games and selection principles
}

\section{Matheus Duzi Ferreira Costa}

Dissertação de Mestrado do Programa de Pós-Graduação em Matemática (PPG-Mat) 

Data de Depósito:

Assinatura:

\title{
Matheus Duzi Ferreira Costa
}

\section{Topological games and selection principles}

\begin{abstract}
Dissertation submitted to the Institute of Mathematics and Computer Sciences - ICMC-USP - in accordance with the requirements of the Mathematics Graduate Program, for the degree of Master in Science. EXAMINATION BOARD PRESENTATION COPY
\end{abstract}

Concentration Area: Mathematics

Advisor: Prof. Dr. Leandro Fiorini Aurichi 
Ficha catalográfica elaborada pela Biblioteca Prof. Achille Bassi e Seção Técnica de Informática, ICMC/USP, com os dados inseridos pelo(a) autor(a)

Duzi, Matheus

Topological games and selection principles /

Matheus Duzi; orientador Leandro Fiorini Aurichi.

- São Carlos, 2019.

$166 \mathrm{p}$.

Dissertação (Mestrado - Programa de Pós-Graduação em Matemática) -- Instituto de Ciências Matemáticas e de Computação, Universidade de São Paulo, 2019.

1. Topological games. 2. Selection principles. 3. Covering properties. 4. Tightness properties. 5. Baire spaces. I. Fiorini Aurichi, Leandro, orient. II. Título. 


\title{
Matheus Duzi Ferreira Costa
}

\section{Jogos topológicos e princípios seletivos}

\author{
Dissertação apresentada ao Instituto de Ciências \\ Matemáticas e de Computação - ICMC-USP, \\ como parte dos requisitos para obtenção do título \\ de Mestre em Ciências - Matemática. EXEMPLAR \\ DE DEFESA \\ Área de Concentração: Matemática \\ Orientador: Prof. Dr. Leandro Fiorini Aurichi
}

USP - São Carlos

Setembro de 2019 

This work is dedicated to all those who do not restrain themselves by the finitude of our earthly lives and, occasionally, decide to venture into the infinite abstract. 

I express my gratitude to FAPESP for the financial support to this project (2017/09797-0).

I thank my advisor Leandro F. Aurichi and everyone from the topology group of ICMC (officially awarded as "The best group of 2018") for all the help throughout these years with the topology and set theory seminars. Here, I particularly thank Henrique A. Lecco, who made the question that motivated the beginning of paper [Aurichi and Duzi 2019].

My thanks also goes to Piotr Szewczak and Boaz Tsaban for giving us access to the preliminary notes of [Szewczak and Tsaban 2019].

Finally, I am specially thankful for the closest friends and family who have stood beside me through the ups and downs of this incredible journey. I would not dare to name all of you, but make no mistake: your support will never be forgotten. 



\section{ABSTRACT}

DUZI, M. Topological games and selection principles. 2019. 166 p. Dissertação (Mestrado em Ciências - Matemática) - Instituto de Ciências Matemáticas e de Computação, Universidade de São Paulo, São Carlos - SP, 2019.

This paper is dedicated to the beginning of the development of a book introducing topological games and selection principles. Here, the classical games (such as the Banach-Mazur) and selection principles (such as the Rothberger or Menger properties) are presented. The most notable applications are also displayed - both the classical (such as the characterization of Baire spaces with the Banach-Mazur game) and the recent (such as the relation between the Menger property and $D$-spaces). In addition to the content for the book, a problem in finite combinatorics that was found in the study of positional strategies is presented (as well as a partial solution) together with some results regarding new variations of classical selection principles and games, which give rise to the characterization of some notable spaces.

Keywords: topological games, selection principles, tightness properties, covering properties, Baire spaces. 



\section{RESUMO}

DUZI, M. Jogos topológicos e princípios seletivos. 2019. 166 p. Dissertação (Mestrado em Ciências - Matemática) - Instituto de Ciências Matemáticas e de Computação, Universidade de São Paulo, São Carlos - SP, 2019.

Este trabalho é dedicado ao início do desenvolvimento de um livro introdutório à jogos topológicos e princípios seletivos. Aqui, são apresentados os clássicos jogos (tais como o de Banach-Mazur) e princípios seletivos (tais como a propriedade de Rothberger ou de Menger). Também são exibidas as aplicações mais notáveis encontradas na literatura - tanto as mais tradicionais (tais como a caracterização de espaços de Baire com o jogo de Banach-Mazur), como as mais atuais (tais como a relação entre a propriedade de Menger e $D$-espaços). Além do conteúdo voltado para o livro, são apresentados um problema de combinatória finita (assim como uma solução parcial para tal) que foi encontrado com o estudo de estratégias posicionais e alguns resultados envolvendo novas variações de princípios de seleção e jogos clássicos, possibilitando a caracterização de alguns espaços notáveis.

Palavras-chave: jogos topológicos, princípios seletivos, propriedades de tightness, propriedades de coberturas, espaços de Baire. 

Introduction . . . . . . . . . . . . . . . . . . 15

I BOOK

$1 \quad$ PRELIMINARIES $\ldots \ldots \ldots \ldots \ldots \ldots \ldots \ldots$

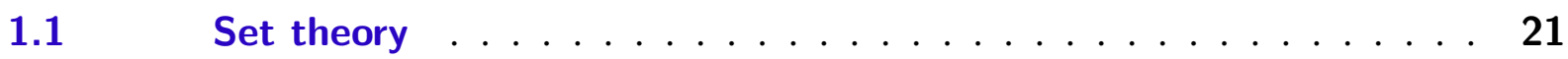

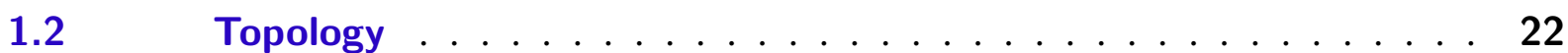

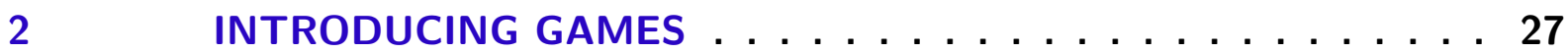

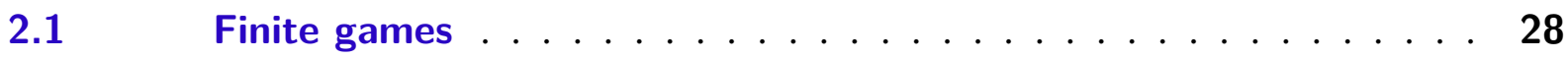

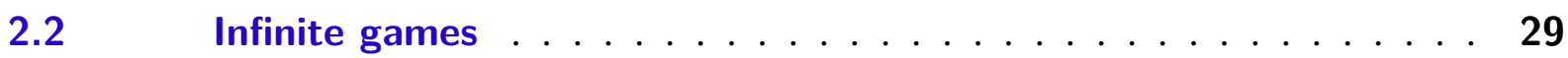

$2.3 \quad$ Strategies . . . . . . . . . . . . . . . . . . 30

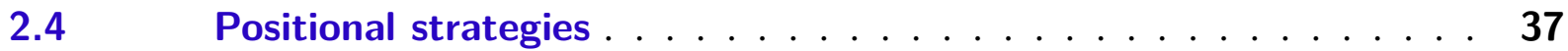

2.5 The Banach-Mazur game . . . . . . . . . . . . . . . . 42

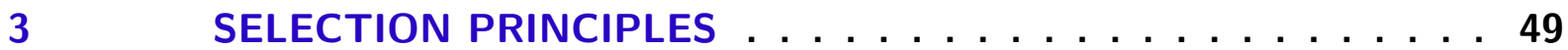

$3.1 \quad$ Classes of selection principles $\ldots \ldots \ldots \ldots \ldots$

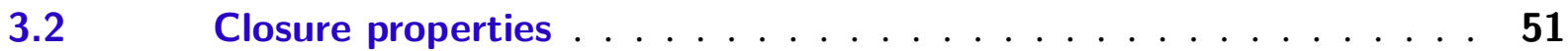

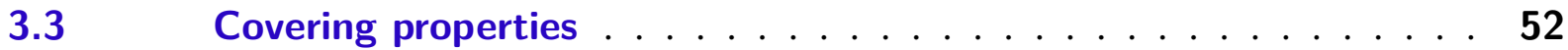

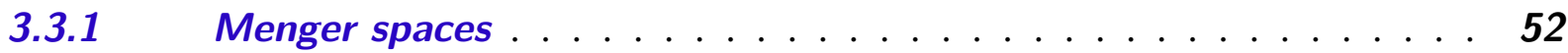

3.3.2 Rothberger spaces . . . . . . . . . . . . . . . . . 53

3.3.3 Hurewicz spaces . . . . . . . . . . . . . . . . . 55

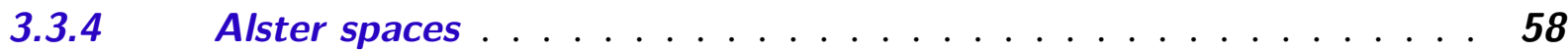

3.3.5 The Scheepers Diagram . . . . . . . . . . . . . . . . . . . . 59

4 THE ASSOCIATED SELECTIVE GAMES $\ldots \ldots \ldots$. . . . 67

$4.1 \quad$ Covering games $\ldots \ldots \ldots \ldots \ldots$

4.1.1 The Rothberger game . . . . . . . . . . . . . . 69

4.1.2 The Menger game. . . . . . . . . . . . . . . . . 72

4.1.3 The Pawlikowski Theorem . . . . . . . . . . . . . . . 81

4.1.4 The Hurewicz game . . . . . . . . . . . . . . . . . . . . . 87

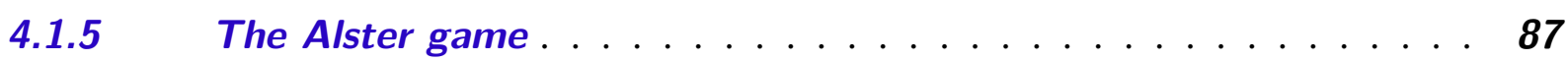

$4.2 \quad$ Closure games $\ldots \ldots \ldots \ldots \ldots \ldots$ 
5 SOME CONNECTIONS AND APPLICATIONS . . . . . . 97

5.1 The Banach-Mazur game and Baire spaces . . . . . . . . . . 97

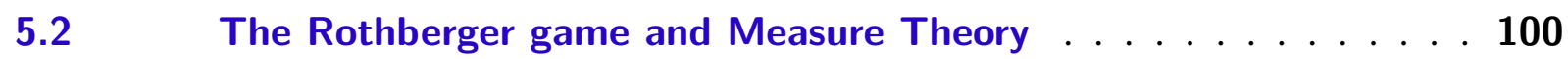

5.2.1 Strong measure zero . . . . . . . . . . . . . . . 100

5.2.2 Purely atomic measures . . . . . . . . . . . . . . . . . 102

$5.3 \quad$ Productively Lindelöf spaces . . . . . . . . . . . . . . . . 105

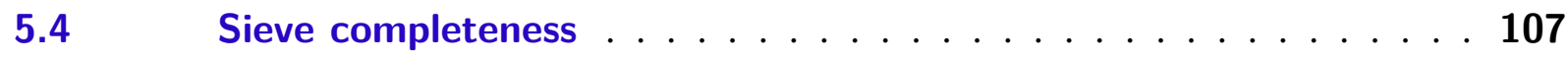

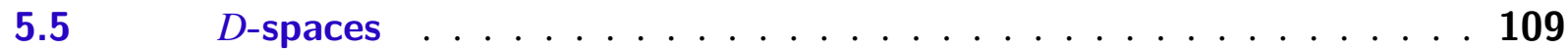

5.6 Tightness games and countable tightness . . . . . . . . . . . . 114

$5.7 \quad$ The space of real continuous functions $\ldots \ldots \ldots \ldots \ldots$

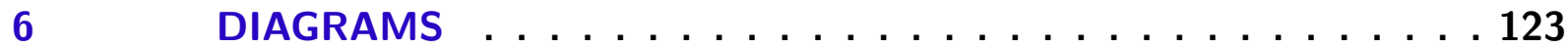

$\begin{array}{lll}\text { II } & \text { RESULTS } & 127\end{array}$

7 POSITIONAL STRATEGIES AND A PROBLEM IN FINITE COMBINATORICS . . . . . . . . . . . . . 129

8 TOPOLOGICAL GAMES OF BOUNDED SELECTIONS $\ldots \ldots 133$

8.1 Selection Principles . . . . . . . . . . . . . . . . 134

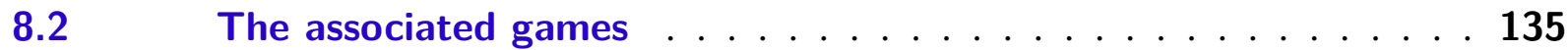

$8.3 \quad$ The "modfin" and "mod 1 " variations . . . . . . . . . . . . . 139

8.4 The analogous to Pawlikowski's and Hurewicz's results . . . . . 147

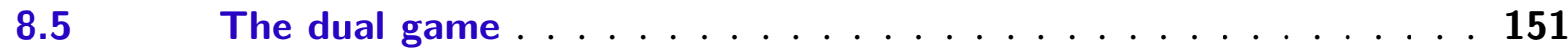

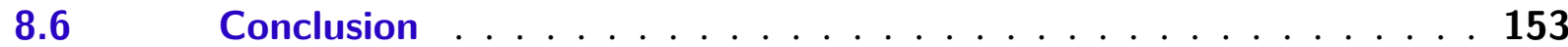

Bibliography . . . . . . . . . . . . . . . . . 159 
Games have been an object of study for mathematicians since, arguably, the 17th century. It was only in the 20th century that, in the Scottish Book, the game henceforth known as the first infinite mathematical game was introduced: the Banach-Mazur game (for a brief history of games in mathematics we refer to [Telgársky 1987] or [Aurichi and Dias 2019]).

Obviously, infinite games are not meant to be played. But what good is a game, if not for playing it? Infinite games are used to describe certain infinite combinatorial properties in an intuitive manner. This description usually happens in terms of winning strategies. The property of being a Baire space, for instance, is characterized by one of the players not having a winning strategy in the Banach-Mazur game, while if the other player has a winning strategy in this game we can go even further and conclude that the space is productively Baire.

Another way to describe combinatorial properties is through selection principles. In essence, these are properties asserting that one can choose an element from each set of a sequence in order to obtain a significant object. Selection principles define classical properties, such as Rothberger or Menger spaces.

Our initial goal was to start the development of a book introducing these infinite games and selection principles in the context of topology. Midway through this project, however, we have obtained some new results on the field, so we decided to divide this text into two parts, each with its respective chapters:

\section{Part I}

This part is dedicated to a prototype of a book on topological games and selection principles.

In Chapter 1 we list the preliminary definitions, results and general notation adopted throughout the book.

With Chapter 2 we formally introduce finite and infinite games, as well as the way we are going to work with them. We particularly focus on exploring the concept of strategies (showing a result regarding positional strategies) while presenting the point-open and the Banach-Mazur games.

We dedicate Chapter 3 to present the general selection principles. Here, we are particularly interested in covering properties (such as Rothberger, Menger and Hurewicz) that rise 
from such principles in order to show the classical Scheepers Diagram, as well as Alster spaces (which we will, later, relate to productively Lindelöf spaces) and closure properties such as fan tightness and strong fan tightness.

In Chapter 4 we introduce the classical selective games associated to the selection principles presented in the previous chapter, as well as their connection with said principles. More specifically, we explore here:

- the duality between the Rothberger game and the point-open game, in addition to its relation to some of its variations and a characterization of Rothberger property;

- the Menger property characterization with the Menger game, as well as its "semi-duality" to the compact-open game;

- a characterization of the Hurewicz property with the introduced Hurewicz game;

- the Alster game duality to the compact- $G_{\delta}$ game and its relation with the compact-open game and the Alster property.

Chapter 5 is dedicated to present some of the classical and recent applications, in addition to some of the connections between the various games and selection principles presented thus far. Namely, we inspect:

- the characterization of Baire spaces with the Banach-Mazur game, as well as how this game relates to productively Baire spaces;

- the characterization of the Rothberger property for metrizable spaces with the concept of strong measure zero and show how regular, $\sigma$-finite Borel measures behave over regular Rothberger spaces.

- a connection between productively Lindelöf spaces and Alster spaces and we present how, assuming the existence of a Michael space, every productively Lindelöf space is Menger;

- a relationship between the compact-open game and sieve completeness when we consider some compactifications;

- the concept of $D$-spaces, as well as its relation with the various covering games presented thus far (such as the Menger game);

- how strong fan tightness and the tightness game relate to productively countably tight spaces;

- how some covering properties naturally translate to tightness properties in the space of real continuous functions. 
Finally, with Chapter 6, we summarize the main results presented in the book into four diagrams.

\section{Part II}

We present in this part the results obtained throughout the project.

In Chapter 7 we talk about how a natural generalization of [Galvin and Telgársky 1986]'s main theorem gave rise to a problem regarding finite combinatorics, for which we present a partial solution.

In Chapter 8 we showcase the content of paper [Aurichi and Duzi 2019], on which we explored how a simple variation of some of the classical selection principles and games may give rise to new topological properties and characterize some spaces. 

Part I

Book 

CHAPTER

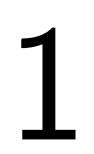

PRELIMINARIES

We dedicate this chapter to clarify some of the notation that will be used throughout this book and to present some usual definitions and results concerning set theory and topology that will be approached.

It should be noted that, since our focus resides in the study of topological games and selection principles, we will leave most of the results appearing here with its proof to be found in other basic topology books (such as [Engelking 1989]).

\subsection{Set theory}

We begin this section by noting that, throughout this entire book, we are assuming the axiomatic system of Zermelo-Fraenkel with the axiom of choice (ZFC). Also, by " $\mathrm{CH}$ " we mean "Continuum Hypothesis".

Definition 1.1.1. We denote the set $\omega \backslash\{0\}$ by $\mathbb{N}$ and the cardinality of the continuum by $\mathfrak{c}$.

Definition 1.1.2. Given a pre-ordered set $\mathbb{P}$, we say two elements $p, q \in \mathbb{P}$ are compatible if there exists $r \in \mathbb{P}$ such that $r \leq p, q$. We say $p, q$ are incompatible, denoted by $\boldsymbol{p} \perp q$, otherwise.

Definition 1.1.3. We define ${ }^{<\omega} \boldsymbol{A}$ as the set of all finite sequences of elements of $A$, that is,

$$
{ }^{<\omega} A=\bigcup_{n \in \omega}^{n} A
$$

where ${ }^{n} A$ is the set of all functions $f: n \rightarrow A$ (in particular, $\emptyset \in{ }^{<\omega} A$ ).

Also, we define ${ }^{\omega} A$ as the set of all functions $f: \omega \rightarrow A$.

To clarify the notation used here:

1. Note that for every $s \in{ }^{<\omega} A, \operatorname{dom}(s)=|s|$. 
2. For $s \in{ }^{<\omega} A$ we write $s=\left\langle s_{0}, \ldots, s_{n}\right\rangle$, with $n=|s|-1$ and $s_{i}=s(i)$ for each $i \leq n<|s|$ (in particular, $\emptyset=\langle\rangle$ );

3. For $s=\left\langle s_{0}, \ldots, s_{n}\right\rangle \in{ }^{<\omega} A$ and $a \in A$, we denote $s^{\frown} a$ as the element $\left\langle s_{0}, \ldots, s_{n}, a\right\rangle \in{ }^{<\omega} A$;

4. For $s \in{ }^{<\omega} A$ and $k \leq|s|$, we denote $s\lceil k$ as simply the function $s$ restricted to $k$.

5. If nothing is said, we will consider ${ }^{<\omega} A$ with the partial order given by

$$
s \geq t \Longleftrightarrow s \subset t
$$

Definition 1.1.4. We say an ordered set $\langle T, \leq\rangle$ is a tree if, for every $t \in T,\{s \in T: s \leq t\}$ is well ordered by $\leq$.

We say $r \in T$ is a root if $\{s \in T: s<t\}=\emptyset$. Given $t \in T$, we say $s \in T$ is a successor of $t$ if $t \leq s$ and there is no $p \in T$ such that $t<p<s$. We say $\langle T, \leq\rangle$ is a finitely branching tree if, for every $t \in T$, the set of successors of $t$ is finite.

Lemma 1.1.5 (König's Lemma). Let $T$ be a finitely branching and infinite tree. Then $T$ has an infinite branch.

Proof. Let $R_{0}$ be $T$ 's set of roots. Note that there is an $r_{0} \in R_{0}$ such that $\left\{t \in T: r_{0} \leq t\right\}$ is infinite. Since the set of successors of $r_{0}$ is finite, one of its successors $r_{1}$ must be such that $\left\{t \in T: r_{1} \leq t\right\}$ is also infinite. By proceeding in this manner we find an infinite branch $R=\left\{r_{n}: n \in \omega\right\}$, as desired.

Definition 1.1.6. Given a set $X$, we say $\mathscr{F} \subset \mathscr{\varrho}(X)$ is a filter base if

- for every $A, B \in \mathscr{F}, A \cap B \in \mathscr{F}$;

- $\emptyset \notin \mathscr{F}$.

\subsection{Topology}

By a space we mean (unless stated otherwise) a nonempty topological space. Formally, a space should be denoted as $\langle X, \tau\rangle$, with $X$ being a set and $\tau$ being the topology in question over $X$. However, $\tau$ will be implicitly clear most of the time, so in these cases we simply write " $X$ " instead of " $\langle X, \tau\rangle$ ".

Definition 1.2.1 (Separation axioms). We say a space $X$ is a

- $\boldsymbol{T}_{1}$ space if, for all $x, y \in X$ such that $x \neq y$, there is an open neighborhood $V_{x}$ of $x$ such that $y \notin V_{x}$ and there is an open neighborhood $V_{y}$ of $y$ such that $x \notin V_{y}$;

- Hausdorff space (or a $\boldsymbol{T}_{\mathbf{2}}$ space) if, for all $x, y \in X$ such that $x \neq y$, there are an open neighborhood $V_{x}$ of $x$ and an open neighborhood $V_{y}$ of $y$ such that $V_{x} \cap V_{y}=\emptyset$; 
- $\boldsymbol{T}_{3}$ space if, for all $x \in X$ and closed $F \subset X$ such that $x \notin F$, there are an open neighborhood $V_{x}$ of $x$ and an open neighborhood $V_{F}$ of $F$ such that $V_{x} \cap V_{F}=\emptyset$;

- regular space if it is $T_{1}$ and $T_{3}$;

- $\boldsymbol{T}_{3 \frac{1}{2}}$ space if, for every closed $F \subset X$ and $x \in X$ such that $x \notin F$ there is a continuous function $f: X \rightarrow[0,1]$ such that $f(x)=0$ and $f(y)=1$ for all $y \in F$;

- completely regular space if it is $T_{1}$ and $T_{3 \frac{1}{2}}$;

- $\boldsymbol{T}_{4}$ space if for all closed and disjoint $F, G \subset X$ there are an open neighborhood $V_{F}$ of $F$ and an open neighborhood $V_{G}$ of $G$ such that $V_{F} \cap V_{G}=\emptyset$;

- normal space if it is $T_{1}$ and $T_{4}$.

Definition 1.2.2. A space $X$ is Lindelöf if all of its open covers have countable subcovers.

Theorem 1.2.3. If $X$ is a $T_{3}$ Lindelöf space, then $X$ is $T_{4}$.

Proof. Let $F$ and $G$ be disjoint closed subsets of a $T_{3}$ Lindelöf space $X$. Since $X$ is $T_{3}$, for each $x \in F$ there is an open neighborhood $V_{x}$ of $x$ such that $\overline{V_{x}} \cap G=\emptyset$. Since $X$ is Lindelöf and $F$ is closed we find a countable subcover $\left\{V_{x_{n}}: n \in \omega\right\} \subset\left\{V_{x}: x \in X\right\}$ for $F$. Analogously, we find a countable cover $\left\{U_{n}: n \in \omega\right\}$ for $G$ such that $\overline{U_{n}} \cap F=\emptyset$ for every $n \in \omega$.

For each $n \in \omega$ let

$$
V_{n}^{*}=V_{n} \backslash \bigcup_{k \leq n} \overline{U_{k}} \text { and } U_{n}^{*}=U_{n} \backslash \bigcup_{k \leq n} \overline{V_{k}}
$$

Note that $\left\{V_{n}^{*}: n \in \omega\right\}$ and $\left\{U_{n}^{*}: n \in \omega\right\}$ are open covers for $F$ and $G$, respectively. After setting $V=\bigcup_{n \in \omega} V_{n}^{*}$ and $U=\bigcup_{n \in \omega} U_{n}^{*}$ we claim that $V \cap U=\emptyset$.

Indeed, note that, for all $n, m \in \omega, V_{n}^{*} \cap U_{m}^{*}=\emptyset$, so

$$
V \cap U=\left(\bigcup_{n \in \omega} V_{n}^{*}\right) \cap\left(\bigcup_{n \in \omega} U_{n}^{*}\right)=\bigcup_{n, m \in \omega}\left(V_{n}^{*} \cap U_{m}^{*}\right)=\emptyset .
$$

Then the proof is complete.

Definition 1.2.4. We say a space $X$ is $\sigma$-compact if there is a collection $\left(K_{n}\right)_{n \in \omega}$ of compact sets such that $X=\bigcup_{n \in \omega} K_{n}$.

Definition 1.2.5. We say that $N \subset \mathbb{R}$ is nowhere dense if the interior of its closure is empty.

Definition 1.2.6. We say $L \subset \mathbb{R}$ is a Luzin set if $L$ is uncountable and $L \cap N$ is countable for every $N$ nowhere dense.

Theorem 1.2.7 (Luzin). CH implies the existence of a Luzin set. 
Proof. Let $\mathscr{F}=\{F \subset \mathbb{R}: F$ is closed and with empty interior $\}$. Since $\mathbb{R}$ has a countable basis, we may write $\mathscr{F}=\left\{F_{\alpha}: \alpha<\omega_{1}\right\}$ with $\mathrm{CH}$. Now, for each $\alpha<\omega_{1}$ we recursively pick

$$
x_{\alpha} \in \mathbb{R} \backslash\left(\left\{x_{\beta}: \beta<\alpha\right\} \cup \bigcup_{\beta \leq \alpha} F_{\beta}\right) .
$$

Note that such $x_{\alpha}$ may always be picked, because $\mathbb{R}$ is a Baire space. We say that $L=$ $\left\{x_{\alpha}: \alpha<\omega_{1}\right\}$ is a Luzin set. Indeed, $L$ is clearly uncountable. Also, if $N \subset \mathbb{R}$ is a nowhere dense subset, then $\bar{N}=F_{\alpha}$ for some $\alpha<\omega_{1}$ and

$$
L \cap N \subset L \cap \bar{N} \subset\left\{x_{\beta}: \beta<\alpha\right\}
$$

and the proof is complete.

Definition 1.2.8. Given a space $X$ and $Y \subset X$, we say a collection of open sets $\mathscr{B}$ is a local basis at $Y$ if

- for every $U \in \mathscr{B}, Y \subset U$;

- for every open $V \supset Y$ there is a $U \in \mathscr{B}$ such that $U \subset V$.

Definition 1.2.9. Given a space $X$ and $Y \subset X$. We say $Y$ has local character of cardinality $\kappa$ if $\kappa$ is the minimum cardinality for a local basis at $Y$. If $\kappa=\omega$, we simply say $Y$ has countable character.

Definition 1.2.10. We say a subspace $Y$ of a space $X$ is a discrete space if for every $y \in Y$ there is an open neighborhood $V_{y}$ of $y$ such that $V_{y} \cap(Y \backslash\{y\})=\emptyset$.

Proposition 1.2.11. If $F_{1}$ and $F_{2}$ are closed discrete subspaces of $X$, then $F_{1} \cup F_{2}$ is a closed discrete subspace of $X$.

Proof. It is clear that $F_{1} \cup F_{2}$ is closed. If $x \in F_{1} \cap F_{2}$, then there are open neighborhoods $V_{x}^{F_{1}}$ and $V_{x}^{F_{2}}$ of $x$ such that $V_{x}^{F_{i}} \cap\left(F_{i} \backslash\{x\}\right)=\emptyset$. In this case, $V_{x}=V_{x}^{F_{1}} \cap V_{x}^{F_{2}}$ is such that $V_{x} \cap$ $\left(\left(F_{1} \cup F_{2}\right) \backslash\{x\}\right)=\emptyset$.

On the other hand, if $x \in F_{i}$ but $x \notin F_{j}$ for distinct $i, j \in\{1,2\}$, then there is an open neighborhood $V_{x}^{\prime}$ of $x$ such that $V_{x}^{\prime} \cap\left(F_{i} \backslash\{x\}\right)=\emptyset$. In this case, if we let $V_{x}=V_{x}^{\prime} \cap\left(X \backslash F_{j}\right)$, it follows that $V_{x} \cap\left(\left(F_{1} \cup F_{2}\right) \backslash\{x\}\right)=\emptyset$.

Definition 1.2.12. We say a family $\mathscr{F}$ of subsets of a space $X$ is a discrete family if for every $x \in X$ there is an open neighborhood $V_{x}$ of $x$ such that $V_{x} \cap F \neq \emptyset$ for at most one $F \in \mathscr{F}$.

Definition 1.2.13. Given a space $X$, we say $\mathscr{F} \subset \mathscr{\mathcal { O }}(X)$ is locally finite at $x \in X$ if there is an open neighborhood $V_{x}$ of $x$ such that $\left\{F \in \mathscr{F}: F \cap V_{x} \neq \emptyset\right\}$ is finite. We say $\mathscr{F}$ is locally finite $X$ if it is locally finite at $x$ for every $x \in X$. 
Example 1.2.14. If $\mathscr{F}$ is a discrete family of subsets of a space $X$, then $\mathscr{F}$ is locally finite in $X$.

Proposition 1.2.15. Let $Y$ be a subspace o a space $X$ and $\mathscr{F} \subset \wp_{(Y)}$ locally finite in $Y$. Then $A=\{x \in X: \mathscr{F}$ is not locally finite at $x\}$ is a closed subset of $X \backslash Y$.

Proof. Since $\mathscr{F}$ is locally finite in $Y, A \subset X \backslash Y$. If $x \in \bar{A}$, then for every open neighborhood $V_{x}$ of $x, V_{x} \cap A \neq \emptyset$. In this case, consider $z \in V_{x} \cap A$. Since $z \in A,\left\{F \in \mathscr{F}: F \cap V_{x} \neq \emptyset\right\}$ is infinite, which implies that $x \in A$ as well and concludes the proof.

Definition 1.2.16. Given a space $X$ and a filter base $\mathscr{F} \subset \mathscr{S}(X)$, we say $\mathscr{F}$ clusters at $x \in X$ if for every $A \in \mathscr{F}$ and $V$ open neighborhood of $x, A \cap V \neq \emptyset$. We say $\mathscr{F}$ clusters in $X$ if there is an $x \in X$ such that $\mathscr{F}$ clusters at $x$.

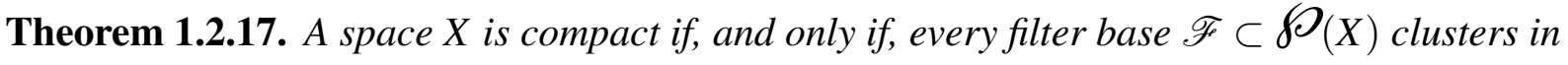
$X$.

Proof. Let $X$ be a compact space and $\mathscr{F} \subset \mathscr{O}(X)$ be a filter base. Then note that $\overline{\mathscr{F}}=$ $\{\bar{F}: F \in \mathscr{F}\}$ has the finite intersection property, so there is an $x \in X$ such that $x \in \bigcap_{F \in F} \bar{F}$. Clearly, $\mathscr{F}$ clusters at $x$.

On the other hand, suppose $X$ is a space such that every filter base $\mathscr{F} \subset \mathscr{}(X)$ clusters in $X$. Let $\mathscr{C}$ be a collection of closed sets with the finite intersection property. Note that

$$
\mathscr{F}=\left\{C_{0} \cap \cdots \cap C_{n}: n \in \omega \text { and } C_{0}, \ldots, C_{n} \in \mathscr{C}\right\}
$$

is a filter base, so it clusters at some point $x \in X$. Since $\mathscr{F}$ 's elements are closed, it follows that $x \in \bigcap \mathscr{F} \subset \bigcap \mathscr{C}$, which concludes the proof.

Definition 1.2.18. Given a completely regular space $X$, we say a space $Y \supset X$ is a compactification of $X$ if $Y$ is a compact Hausdorff space and $\bar{X}=Y$.

Theorem 1.2.19. The space of irrational numbers is homeomorphic to $\mathbb{N}^{\omega}$. 



\section{INTRODUCING GAMES}

The reader would find quite strange to read a book with "topological games" in the title that does not even once mentions game theory. We should emphasize here, however, that topology is the actual central object of study of this book and we focus only on specific kinds of games.

Precisely, we are interested mainly in two players competing and sequential games of perfect information that do not allow draws. These are game theory's classification terms which we briefly and informaly explain in what follows:

- two players: as obvious as it sounds, the game is played between two players, which we will call ALICE and BOB;

- competing: players compete, rather than cooperate towards some goal;

- sequential: players take actions in turns, rather than simultaneously;

- perfect information: at every given moment of the game, both players have knowledge of the entire history of the game thus far;

- does not allow draws: at the end of a run of the game, one of the players wins and the other loses.

Surely, for each of the categories presented above one can find some example of games that are playable in real life (such as chess, checkers, tic-tac-toe, etc.). However, in order to study topological properties, we will not limit ourselves to study such playable games that finish in finitely many moves: so we rely on the abstract mathematics, striving for the study of infinite games. But before we introduce such abstract games, we take a step back and formalize the case of finite games, so that the concept of infinite games falls more, let us say, naturally to the reader. 


\subsection{Finite games}

Sequential games, in particular, are known to have an extensive-form, which is a representation of such games using trees. We use this representation to later naturally extend to infinite games:

Definition 2.1.1. Let $M$ be a nonempty set and $N \in \mathbb{N}$. Given $T \subset{ }^{<2 N+1} M$ and $A \subset{ }^{2 N+1} M$, we say $\mathrm{G}=\langle T, A\rangle$ is a finite game played between ALICE and BOB of length $N$ if $T$ satisfies the following conditions:

(I) \langle\rangle$\in T$;

(II) If $t \in T$, then $t\lceil k \in T$ for every $k \leq|t|$;

(III) For every $t \in T$ such that $|t|<2 N$ there is an $x \in M$ such that $t^{\frown} x \in T$.

An element $t \in T$ is called a moment of the game G. An element $R \in{ }^{2 N} M$ is called a run of the game $\mathrm{G}$ if $R\lceil n \in T$ for every $n \leq 2 N$.

If a moment $t$ has even length, we say it is ALICE's turn and $\left\{x \in M: t^{\curvearrowleft} x \in T\right\}$ is the set of ALICE's valid responses to $t$ in the game G. Likewise, if a moment $t$ has odd length, we say it is BOB's turn and $\left\{x \in M: t^{\frown} x \in T\right\}$ is the set of BOB's possible responses to $t$ in the game G.

We then fix the following notations

$$
\begin{aligned}
& \mathrm{A}(\mathrm{G})=\left\{x \in M: t^{\imath} x \in T \text { for some } t \in T \text { which is AlicE's turn }\right\} \\
& \mathrm{B}(\mathrm{G})=\left\{x \in M: t^{\frown} x \in T \text { for some } t \in T \text { which is BoB's turn }\right\}
\end{aligned}
$$

If $t \in T$ is a moment such that $|t|=2 k$ or $|t|=2 k+1$ for some $k \in \omega$, then we say $t$ is at the inning $k$.

Finally, $A$ is called the payoff set of the game G. A run $R$ is said to be won by ALICE if $R \in A$. Otherwise, we say $R$ is a run won by Вов.

The motivation for defining games as in Definition 2.1.1 is as follows:

- Condition I makes sure the game has a start (here, the empty sequence \langle\rangle represents the start of the game);

- Condition II makes sure every moment of the game can be attained if ALICE and BoB play the game from the start in some way;

- Condition III makes sure the game ends only once it "reaches" length $2 N$ (this is purely for technical reasons). 


\subsection{Infinite games}

Once we understand the meanings of Definition 2.1.1, we can naturally extend it to infinite games preserving the same interpretations:

Definition 2.2.1 (Infinite Game). Let $M$ be a nonempty set. Given $T \subset{ }^{<\omega} M$ and $A \subset{ }^{\omega} M$, we say $\mathrm{G}=\langle T, A\rangle$ is an infinite game between ALICE and Bов if $T$ satisfies the following conditions:

(I) \langle\rangle$\in T$;

(II) If $t \in T$, then $t\lceil k \in T$ for every $k \leq|t|$;

(III) For every $t \in T$ there is an $x \in M$ such that $t^{\curvearrowleft} x \in T$.

An element $t \in T$ is called a moment of the game $\mathrm{G}$. An element $R \in{ }^{\omega} M$ is called a run of the game $\mathrm{G}$ if $R\lceil n \in T$ for every $n \in \omega$.

If a moment $t$ has even length, we say it is ALICE's turn and $\left\{x \in M: t^{\frown} x \in T\right\}$ is the set of ALICE's valid responses to $t$ in the game G. Likewise, if a moment $t$ has odd length, we say it is BоB's turn and $\left\{x \in M: t^{\frown} x \in T\right\}$ is the set of BOB's possible responses to $t$ in the game G.

We then fix the following notations

$$
\begin{aligned}
& \mathrm{A}(\mathrm{G})=\left\{x \in M: t^{\curlywedge} x \in T \text { for some } t \in T \text { which is AliCE's turn }\right\} \\
& \mathrm{B}(\mathrm{G})=\left\{x \in M: t^{\frown} x \in T \text { for some } t \in T \text { which is BoB's turn }\right\}
\end{aligned}
$$

If $t$ is a moment and $|t|=2 k$ or $|t|=2 k+1$ for some $k \in \omega$, then we say $t$ is at the inning $k$.

Finally, $A$ is called the payoff set of the game G. A run $R$ is said to be won by ALICE if $R \in A$. Otherwise, we say $R$ is a run won by Bов.

The motivation for defining games as in Definition 2.2.1 is, then, analogous to the motivation presented for finite games - in this case, Condition III makes sure the game only ends once it "reaches infinity" (again, this is purely for technical reasons).

The games we are going to work with are usually defined by rules (just like real life games). These so called rules are usually recursions that define the tree of the game and a formula that defines the payoff set. For instance:

Example 2.2.2. Fix $A \subset{ }^{\omega} \omega$ and consider the following game $G$. At each inning $n \in \omega$, ALICE chooses $a_{n} \in \omega$ and BOB responds with $b_{n} \in \omega$. ALICE then wins the game if $\left\langle a_{0}, b_{0}, \ldots, a_{n}, b_{n}, \ldots\right\rangle \in$ $A$. Formally speaking, these rules define the game $\mathrm{G}=\left\langle{ }^{<\omega} \omega, A\right\rangle$. 
Example 2.2.3 (Point-open game). Given a space $X$ we call the point-open game on $X$ the following game: in each inning $n \in \omega$,

- AliCE chooses a point $x_{n} \in X$;

- BoB then responds with an open neighborhood $V_{n}$ of $x_{n}$.

We say ALICE wins if $X=\bigcup_{n \in \omega} V_{n}$.

Example 2.2.3 is our first example of a topological game! Intuitively, a topological game is a set of rules that defines a game for each given space. Another example of a topological game can be obtained as a simple variation of the point-open game:

Example 2.2.4 (Finite-open game). Given a space $X$ we call the finite-open game on $X$ the following game: in each inning $n \in \omega$,

- Alice chooses $F_{n} \subset X$ finite;

- B Ов then responds with an open set $V_{n}$ such that $F_{n} \subset V_{n}$.

We say ALICE wins if $X=\bigcup_{n \in \omega} V_{n}$.

As we will see in the next section, however, the point-open and the finite-open games are not much different from one another, in some sense.

\subsection{Strategies}

Strategies will play a key role in our studies of topological games, so let us formally define what a strategy actually is in view of what we defined as a game.

Definition 2.3.1 (Strategy for ALICE). A strategy for ALICE in a game $G=\langle T, A\rangle$ is a function $\gamma: S \rightarrow \mathrm{A}(\mathrm{G})$, with $S \subset T$, that satisfies the following conditions

(a) \langle\rangle$\in S$;

(b) if $s \in S$, then $s$ is ALICE's turn;

(c) for every $s \in S, s^{\urcorner} \gamma(s) \in T$;

(d) for each $s \in S, s^{\frown} y^{\frown} x \in S$ if, and only if, $y=\gamma(s)$ and $x$ is a possible response of BoB to the sequence $s^{\frown} \gamma(s)$. 
Intuitively, a strategy $\gamma$ for ALICE is a function whose input is the history of the game so far at a given moment and the output is a valid response of ALICE to such history - it tells ALICE exactly how she will play the game. Condition (a) is assuring that $\gamma$ tells ALICE how to start the game, (b) tell us that $\gamma$ takes into account only turns that actually correspond to ALICE's turn, (c) assures that $\gamma$ tells ALICE to play only valid responses and condition (d) makes sure $\gamma$ prepares ALICE to deal with every possible situation she might get into by playing according to $\gamma$.

We note that a strategy $\gamma$ for ALICE in $\mathrm{G}=\langle T, A\rangle$ may be uniquely identified with a subtree $T(\gamma) \subset T$. This tree is easily obtained from $\gamma$ itself: just let $t \in T(\gamma)$ if, and only if, $t \in \operatorname{dom}(\gamma)$ or $t=s^{\wedge} \gamma(s)$ for some $s \in \operatorname{dom}(\gamma)$. A run $R$ is then said to be compatible with an ALICE's strategy $\gamma$ if $R\lceil n \in T(\gamma)$ for every $n \in \omega$ (basically, if the run $R$ can be played by ALICE following the instructions of strategy $\gamma$ ).

To simplify the notation, if $\gamma$ is a strategy for ALICE and $s$ is in its domain, we omit the even numbered entries of the sequence $s$ - in view of condition (d), these entries are already implicitly determined by $\gamma$ itself.

We say $\gamma$ is a winning strategy for ALICE if for every run $R$ compatible with $\gamma, R$ is won by ALICE. We denote the assertion "AlICE has a winning strategy in G" with "ALICE $\uparrow$ G" and the assertion "ALICE has no winning strategy in G” with “ALICE $\Varangle G ”$.

Example 2.3.2. If $A={ }^{\omega} \omega$ and we consider the game $\mathrm{G}=\left\langle{ }^{<\omega} \omega, A\right\rangle$, then ALICE $\uparrow \mathrm{G}$. In fact every strategy for ALICE is a winning one!

Example 2.3.3. If $X$ is a countable space, then ALICE has an obvious winning strategy in the point-open game: she can just pick each and every point of the space along one run.

We then, analogously, define a strategy for Bов:

Definition 2.3.4 (Strategy for Bов). A strategy for Bов in a game $G=\langle T, A\rangle$ is a function $\sigma: S \rightarrow \mathrm{B}(G)$, with $S \subset T$, that satisfies the following conditions

(a) $\langle x\rangle \in S$ for each valid starting move $x$ of ALICE;

(b) if $s \in S$, then $s$ is BoB's turn;

(c) for every $s \in S, s^{\wedge} \sigma(s) \in T$;

(d) for each $s \in S, s^{\urcorner} y^{\urcorner} x \in S$ if, and only if, $y=\sigma(s)$ and $x$ is a possible response of ALICE to the sequence $s^{\wedge} \sigma(s)$.

Just like for ALICE's strategies, we uniquely identify a strategy $\sigma$ with a subtree $T(\sigma) \subset$ $T$ in the same manner: by letting $t \in T(\sigma)$ if, and only if, $t \in \operatorname{dom}(\sigma)$ or $t=s^{\wedge} \sigma(s)$ for some $s \in \operatorname{dom}(\sigma)$. Again, a run $R$ is said to be compatible with a strategy $\sigma$ for Вов if $R\lceil n \in T(\sigma)$ for every $n \in \omega$. 
Also, if $\sigma$ is a strategy for Вов and $s$ is in its domain, we omit the odd numbered entries of the sequence $s$ - again, in view of condition (d), these entries are already implicitly determined by $\sigma$ itself.

We say $\sigma$ is a winning strategy for Вов if for every run $R$ compatible with $\sigma, R$ is won by Вов. We denote the assertion "Вов has a winning strategy in G" with "Вов $\uparrow$ G" and the assertion "Bов has no winning strategy in G" with "Bов $\Varangle G$ ".

Example 2.3.5. If $A=\emptyset$ and we consider the game $\mathrm{G}=\left\langle{ }^{<\omega} \omega, A\right\rangle$, then $\mathrm{BOB} \uparrow \mathrm{G}$. In fact every strategy for Вов is a winning one!

Example 2.3.6. If $X=\mathbb{R}$, then В Ов has a winning strategy in the point-open game: all he has to do is to respond in each inning $n \in \omega$ with an open interval of length $\frac{1}{2^{n}}$. The union of his responses will then have Lebesgue measure $M \leq \sum_{n=0}^{\infty} \frac{1}{2^{n}}=2$, and, therefore, will not cover $\mathbb{R}$ (note that the same argument can be used to show that Вов has a winning strategy in the point-open game on $X$ if $X \subset \mathbb{R}$ has infinite Lebesgue measure).

Example 2.3.7. If $X \subset \mathbb{R}$ has finite Lebesgue measure $M>0$, then B OB has a winning strategy in the point-open game just like in Example 2.3.6 by choosing, in each inning n, intervals of length $\frac{M}{2} \frac{1}{2^{n}}$ this time.

As the reader might have already figured out from Examples 2.3.3, 2.3.6 and 2.3.7, we usually define strategies recursively. Eventually, these recursions might get way too complicated in a way that writing them formally might get in the way of understanding what is going on in the construction of said strategy. When that is the case, we refrain ourselves from writing the formal recursion and we just show the beginning of the process, ending the construction with something like "and so on". Of course, when doing that we expect that if one wants to actually write the formal recursion, they may easily (although messily) do it with the help of our "informal" recursion.

Also, note that the set of ALICE's strategies (or BoB's) for a given game $\mathrm{G}=\langle T, A\rangle$ does not depend on the payoff set $A$ (the winning criteria of the game), only on the tree $T$ (the set of restriction on the moves of the players). The payoff set $A$ only dictates which strategies are winning or not. Formally:

Lemma 2.3.8. Let $\mathrm{G}_{1}=\left\langle T, A_{1}\right\rangle$ and $\mathrm{G}_{2}=\left\langle T, A_{2}\right\rangle$. If $\Gamma\left(\mathrm{G}_{1}\right)$ is the set of strategies for ALICE in $\mathrm{G}_{1}$ and $\Gamma\left(\mathrm{G}_{2}\right)$ is the set of strategies for ALICE in $\mathrm{G}_{2}$, then

$$
\Gamma\left(\mathrm{G}_{1}\right)=\Gamma\left(\mathrm{G}_{2}\right)
$$

Analogously, if $\Sigma\left(\mathrm{G}_{1}\right)$ is the set of strategies for $\mathrm{B}$ ов in $\mathrm{G}_{1}$ and $\Sigma\left(\mathrm{G}_{2}\right)$ is the set of strategies for BoB in $\mathrm{G}_{2}$, then

$$
\Sigma\left(G_{1}\right)=\Sigma\left(G_{2}\right)
$$


Since our games do not allow draws, one may inadvertently conclude that if one of the players have no winning strategy then the other must have one. This is, however, NOT the case (in general), which is why we present the following definition.

Definition 2.3.9. We say a game $G$ is

- determined if ALICE $\uparrow \mathrm{G}$ or BOB $\uparrow \mathrm{G}$;

- undetermined otherwise, i.e., if ALICE $\Varangle G$ and BOB $\Varangle G$.

Indeed, finite games are all determined:

Theorem 2.3.10 ([Zermelo 1913], [Kalmár 1928]). If $\mathrm{G}=\langle T, A\rangle$ is a finite game of length $N \in \omega$, then $\mathrm{G}$ is determined.

Proof. Note that ALICE $\uparrow$ G means

$$
\exists a_{1} \forall b_{1} \exists a_{2} \forall b_{2} \ldots \exists a_{N} \forall b_{N} \text { ALICE wins the run }\left\langle a_{1}, b_{1}, \ldots, a_{N}, b_{N}\right\rangle \text {. }
$$

So ALICE $\Varangle G$ means

$\forall a_{1} \exists b_{1} \forall a_{2} \exists b_{2} \ldots \forall a_{N} \exists b_{N}$ such that ALICE does not win the run $\left\langle a_{1}, b_{1}, \ldots, a_{N}, b_{N}\right\rangle$,

which means exactly that $\mathrm{BoB} \uparrow \mathrm{G}$.

When we are talking about infinite games, on the other hand, we may have a very different story. Note that in the proof of Proposition 2.3.10 we strongly used the fact that the game $\mathrm{G}$ had a finite set of innings, so it might not be a surprise to know that, for instance:

Theorem 2.3.11 ([Khomskii 2010]). There is an $A \subset{ }^{\omega} \omega$ under ZFC such that $\mathrm{G}=\left\langle{ }^{<\omega} \omega, A\right\rangle$ is undetermined.

Proof. In view of Lemma 2.3.8, we may let $\Gamma$ be the set of ALICE's strategies and $\Sigma$ be the set of BoB's strategies in $\mathrm{G}=\left\langle{ }^{<\omega} \omega, A\right\rangle$, for every $A \subset{ }^{\omega} \omega$. Then, for each $\gamma \in \Gamma$, let $\mathscr{R}(\gamma)$ be the set of runs that are compatible with $\gamma$. Analogously, for each $\sigma \in \Sigma$, let $\mathscr{R}(\sigma)$ be the set of runs that are compatible with $\sigma$. Note that

$$
|\Gamma|=|\Sigma|=|\mathscr{R}(\gamma)|=|\mathscr{R}(\sigma)|=\mathfrak{c}
$$

for every $\gamma \in \Gamma$ and $\sigma \in \Sigma$. Then we let $\Gamma=\left\{\gamma_{\xi}: \xi<\mathfrak{c}\right\}$ and $\Sigma=\left\{\sigma_{\xi}: \xi<\mathfrak{c}\right\}$. Now we can define $A \subset{ }^{\omega} \omega$ and $B \subset{ }^{\omega} \omega$ with the following recursion: First, pick any $R_{0} \in \mathscr{R}\left(\sigma_{0}\right)$ and then $S_{0} \in \mathscr{R}\left(\gamma_{0}\right)$ such that $S_{0} \neq R_{0}$. Now, suppose that, for some $\alpha<\mathfrak{c}$, we have defined $R_{\xi}$ and $S_{\xi}$ for every $\xi<\alpha$ in such a way that, for every $\xi, \eta<\alpha$,

- $R_{\xi} \in \mathscr{R}\left(\sigma_{\xi}\right)$ and $S_{\xi} \in \mathscr{R}\left(\gamma_{\xi}\right)$; 
- $S_{\xi} \neq R_{\eta}$.

Then pick $R_{\alpha} \in \mathscr{R}\left(\sigma_{\alpha}\right)$ and $S_{\alpha} \in \mathscr{R}\left(\gamma_{\alpha}\right)$ such that $R_{\alpha} \notin\left\{S_{\xi}: \xi<\alpha\right\}$ and $S_{\alpha} \notin\left\{R_{\xi}: \xi \leq \alpha\right\}$ (this is possible, because $\left|\mathscr{R}\left(\sigma_{\alpha}\right)\right|=\left|\mathscr{R}\left(\gamma_{\alpha}\right)\right|=\mathfrak{c}$ ). Let $A=\left\{R_{\xi}: \xi<\mathfrak{c}\right\}$ and $B=\left\{S_{\xi}: \xi<\mathfrak{c}\right\}$. Clatm 2.3.12. $A \cap B=\emptyset$.

Proof. Let $R \in A$. Then $R=R_{\alpha}$ for some $\alpha<\mathfrak{c}$. Note that at stage $\alpha$ of our recursion, $R_{\alpha}$ was picked in such a way that $R_{\alpha} \neq S_{\xi}$ for every $\xi<\alpha$. But then, for every $\eta$ such that $\alpha \leq \eta<\mathfrak{c}$, $S_{\eta}$ was picked in such a way that $S_{\eta} \neq R_{\xi}$ for every $\xi<\eta$. In particular, $R_{\alpha} \neq S_{\eta}$ for every $\eta \geq \alpha$, and the proof is complete.

Finally, we claim that $\mathrm{G}=\left\langle{ }^{<\omega} \omega, A\right\rangle$ is undetermined. Indeed, let $\gamma \in \Gamma$. Then $\gamma=\gamma_{\alpha}$ for some $\alpha<\mathfrak{c}$ and $S_{\alpha} \in \mathscr{R}\left(\gamma_{\alpha}\right) \cap B$, so it follows from Claim 2.3.12 that ALICE loses the run $S_{\alpha}$ and, therefore, $\gamma$ is not a winning strategy. On the other hand, if $\sigma \in \Sigma, \sigma=\sigma_{\alpha}$ for some $\alpha<\mathfrak{c}$ and $R_{\alpha} \in \mathscr{R}\left(\sigma_{\alpha}\right) \cap A$, which means that $\mathrm{B}$ ов loses the run $R_{\alpha}$ and, therefore, $\sigma$ is not a winning strategy.

Now that we know that infinite games may not be determined, we may ask:

Question 2.3.13. Is the point-open game determined on every subset of $\mathbb{R}$ ?

Indeed, as we will see later, the answer to Question 2.3.13 is actually independent of ZFC.

For now, we present a definition that should indicate how important the concept of strategies will be in our studies of topological games: given a class of spaces $\mathscr{C}$ (like the class of all Hausdorff spaces, for instance), we say two topological games $G_{1}$ and $G_{2}$ are equivalent over $\mathscr{C}$ if, for every $X \in \mathscr{C}$,

- AlICE has a winning strategy in $G_{1}$ on $X$ if, and only if, ALICE has a winning strategy in $\mathrm{G}_{2}$ on $X$;

- Вов has a winning strategy in $\mathrm{G}_{1}$ on $X$ if, and only if, Bов has a winning strategy in $\mathrm{G}_{2}$ on $X$.

If $G_{1}$ and $G_{2}$ are equivalent over the class of all spaces, we simply say that $G_{1}$ and $G_{2}$ are equivalent games.

Proposition 2.3.14. The point-open game and the finite-open game are equivalent.

Proof. It is clear that if ALICE has a winning strategy $\gamma$ in the point-open game, then ALICE has a winning strategy in the finite-open game (she can use a restriction of the same $\gamma$ ) and that if 
Вов has a winning strategy $\sigma$ in the finite-open game, then Вов has a winning strategy in the point-open game (again, he can use a restriction of the same $\sigma$ ).

So suppose $\gamma$ is a winning strategy for ALICE in the point-open game. We define a strategy $\gamma^{\prime}$ for ALICE in the finite-open game as follows:

- Let $\gamma(\langle\rangle)=\left\{x_{j}: j \leq n_{0}\right\}$ and then, in the first inning, let $\gamma^{\prime}(\langle\rangle)=x_{0}$. Regardless of what open set $V_{0}$ ВОВ responds with, let $\gamma^{\prime}\left(\left\langle V_{0}\right\rangle\right)=x_{1}$. In fact, let $\gamma^{\prime}\left(\left\langle V_{i}: i<j\right\rangle\right)=x_{j}$ in every inning $j \leq n_{0}$ (regardless of what BOB's responses are);

- Let $U_{0}=\bigcup_{j \leq n_{0}} V_{j}$ and note that $\gamma(\langle\rangle) \subset U_{0}$, so we may set $\gamma\left(\left\langle U_{0}\right\rangle\right)=\left\{x_{j}: n_{0}<j \leq n_{1}\right\}$. Then, in the inning $n_{0}+1$, let $\gamma^{\prime}\left(\left\langle V_{j}: j<n_{0}+1\right\rangle\right)=x_{n_{0}+1}$. Regardless of what open set $V_{n_{0}+1}$ ВОВ responds with, let $\gamma^{\prime}\left(\left\langle V_{j}: j<n_{0}+2\right\rangle\right)=x_{n_{0}+1}$. In fact, let $\gamma^{\prime}\left(\left\langle V_{i}: i \leq j\right\rangle\right)=x_{j}$ in every inning $j$ such that $n_{0}<j \leq n_{1}$ (regardless of what BоB's responses are);

- In the inning $n_{1}+1$, let $U_{1}=\bigcup_{n_{0}<j \leq n_{1}} V_{j}$ and note that $\gamma\left(\left\langle U_{0}\right\rangle\right) \subset U_{1}$, so we may set $\gamma\left(\left\langle U_{0}, U_{1}\right\rangle\right)=\left\{x_{j}: n_{1}<j \leq n_{2}\right\}$. Then, let $\gamma^{\prime}\left(\left\langle V_{j}: j<n_{1}+1\right\rangle\right)=x_{n_{1}+1}$. Regardless of what open set $V_{n_{1}+1}$ BOB responds with, let $\gamma^{\prime}\left(\left\langle V_{j}: j<n_{1}+2\right\rangle\right)=x_{n_{1}+1}$. In fact, let $\gamma^{\prime}\left(\left\langle V_{i}: i \leq j\right\rangle\right)=x_{j}$ in every inning $j$ such that $n_{1}<j \leq n_{2}$ (regardless of what BoB's responses are);

- and so on.

It follows that the open sets chosen by Вов in a run against $\gamma^{\prime}$ cover the same as the open sets chosen by Вов in a run against $\gamma$, so $\gamma^{\prime}$ must be a winning strategy.

Now, suppose $\sigma$ is a winning strategy for Вов in the point-open game. We define a strategy $\sigma^{\prime}$ for Вов in the finite-open game as follows:

- If ALICE starts with $\mathscr{F}_{0}=\left\{x_{j}: j \leq n_{0}\right\}$, let

$$
\sigma^{\prime}\left(\left\langle\mathscr{F}_{0}\right\rangle\right)=\bigcup_{k \leq n_{0}} \sigma\left(\left\langle x_{j}: j \leq k\right\rangle\right)
$$

- if in the next inning ALICE chooses $\mathscr{F}_{1}=\left\{x_{j}: n_{0}<j \leq n_{1}\right\}$, let

$$
\sigma^{\prime}\left(\left\langle\mathscr{F}_{0}, \mathscr{F}_{1}\right\rangle\right)=\bigcup_{n_{0}<k \leq n_{1}} \sigma\left(\left\langle x_{j}: j \leq k\right\rangle\right)
$$

- next, if ALICE chooses $\mathscr{F}_{2}=\left\{x_{j}: n_{1}<j \leq n_{2}\right\}$, let

$$
\sigma^{\prime}\left(\left\langle\mathscr{F}_{0}, \mathscr{F}_{1}\right\rangle\right)=\bigcup_{n_{1}<k \leq n_{2}} \sigma\left(\left\langle x_{j}: j \leq k\right\rangle\right)
$$

- and so on. 
It follows that the open sets chosen by $\sigma^{\prime}$ in a run cover the same as the open sets chosen by $\sigma$ in a run in the point-open game, so $\sigma^{\prime}$ must be a winning strategy.

We can go even further regarding ALICE having a winning strategy in the finite-open game. In order to do this, consider yet another variation of the point-open game:

Example 2.3.15. Given a space $X$ we call the strict finite-open game on $X$ the following game: in each inning $n \in \omega$,

- Alice chooses $F_{n} \subset X$ finite;

- BoB then responds with an open set $V_{n}$ such that $F_{n} \subset V_{n}$.

We say ALICE wins if $X=\bigcup_{n \in \omega} \bigcap_{k \geq n} V_{k}$.

The difference between the finite-open game and the strict finite-open game is that the latter's winning criteria is made harder for ALICE to achieve than the former's: in the strict finite-open game she not only has to make sure the entire space gets covered, but she also has to make sure each point of the space is in all but finitely many of the open sets chosen by Вов in a run. With this in mind, one could assume that ALICE having a winning strategy in the finite-open game does not imply she has one in the strict variation. Surprisingly, though, it does imply:

Theorem 2.3.16 ([Gruenhage 1976]). The following properties are equivalent on every space $X$ :

- ALICE has a winning strategy in the point-open game;

- ALICE has a winning strategy in the strict finite-open game.

Proof. Let $\gamma$ be a winning strategy for ALICE in the point-open game. Then we define a strategy $\tilde{\gamma}$ for ALICE in the strict finite-open game as follows.

- First, we let

$$
\tilde{\gamma}(\langle\rangle)=\gamma(\langle\rangle)
$$

- If Bов then chooses an open $V_{0}$ such that $V_{0} \supset \tilde{\gamma}(\langle\rangle)$, we let

$$
\tilde{\gamma}\left(\left\langle V_{0}\right\rangle\right)=\gamma(\langle\rangle) \cup \gamma\left(\left\langle V_{0}\right\rangle\right)
$$

- If B ов then chooses an open $V_{1}$ such that $V_{1} \supset \tilde{\gamma}\left(\left\langle V_{0}\right\rangle\right)$, we let

$$
\tilde{\gamma}\left(\left\langle V_{0}, V_{1}\right\rangle\right)=\gamma(\langle\rangle) \cup \gamma\left(\left\langle V_{0}\right\rangle\right) \cap \gamma\left(\left\langle V_{1}\right\rangle\right) \cap \gamma\left(\left\langle V_{0}, V_{1}\right\rangle\right)
$$


- In general, if Bов chooses an open $V_{n}$ such that $V_{n} \supset \tilde{\gamma}\left(\left\langle V_{i}: i<n\right\rangle\right)$ we let $S$ be the (finite) collection of subsequences of $\left\langle V_{i}: i \leq n\right\rangle$ and then

$$
\tilde{\gamma}\left(\left\langle V_{i}: i<n\right\rangle^{\urcorner} V_{n}\right)=\bigcup_{s \in S} \gamma(s)
$$

Now, let $\left\langle V_{n}: n \in \omega\right\rangle$ be a run compatible with $\tilde{\gamma}$ (we are omitting ALICE's moves in the run). Note that, in order to show that ALICE wins in this run, it suffices to show that $X=$ $\bigcup_{i \in I} V_{i}$ for every infinite $I \subset \omega$. In this case, let $I \subset \omega$ be an arbitrary infinite set and fix an increasing enumeration $I=\left\{i_{k}: k \in \omega\right\}$. Then, because of the way we constructed $\tilde{\gamma}$, the sequence $\left\langle V_{i_{k}}: k \in \omega\right\rangle$ can be played against $\gamma$. Hence, $\bigcup_{k \in \omega} V_{i_{k}}=X$ and $\tilde{\gamma}$ is a winning strategy.

The other implication is trivial.

Exercise 2.3.17. Show that ALICE and BOB cannot both have a winning strategy in a game G.

\subsection{Positional strategies}

It should be noted that the fact that two games are equivalent does not imply they share the same properties. For instance, consider the following game:

Definition 2.4.1. Given a space $X$ we call the increasing point-open game on $X$ the following game: in each inning $n \in \omega$,

- Alice chooses a point $x_{n} \in X \backslash V_{n-1}$;

- Bов then responds with an open neighborhood $V_{n}$ of $x_{n}$ such that $V_{n} \supset V_{n-1}$.

We say AliCE wins if $X=\bigcup_{n \in \omega} V_{n}$.

It is a simple exercise to show that the point-open game is then equivalent to the increasing point-open game. The latter, however, has a stronger property not shared by the former:

Theorem 2.4.2 ([Galvin and Telgársky 1986]). If ALICE has a winning strategy in the increasing point-open game on a space $X$, then ALICE has a winning strategy $\gamma^{*}$ such that for every $t \in \operatorname{dom}\left(\gamma^{*}\right)$ and open $V$ with $\gamma^{*}(t) \in V$,

$$
\gamma^{*}\left(t^{\frown} V\right)=\gamma^{*}(\langle V\rangle)
$$

Proof. Let $\langle X, \tau\rangle$ be a space, $\tau^{*}=\tau \backslash\{\emptyset\}$ and $\gamma$ be a winning strategy for ALICE in the increasing point-open game. Let $P=\operatorname{dom}(\gamma)$ and, given $V \in \tau^{*}$, let $P(V)=\left\{t=\left\langle V_{k}: k \leq n\right\rangle \in P: V_{n}=V\right\}$. Let $<$ be a fixed (strict) well order over $\tau^{*}$, so that $<$ induces the following strict linear order over $P$ : $s \prec t$ if either $t \subset s$ or else there is a $k \in \operatorname{dom}(s) \cap \operatorname{dom}(t)$ such that $s\lceil k=t\lceil k$ and $s(k+1)<t(k+1)$ (this is similar to the lexicographic order). 
CLAIM 2.4.3. If $\left\langle t_{n}: n \in \omega\right\rangle$ is a strictly decreasing sequence with respect to $\prec$, then for every $k \in \omega$ there is an $N_{k} \in \omega$ such that $k \subset \operatorname{dom}\left(t_{n}\right)$ for every $n \geq N_{k}$.

Proof. We will prove this by induction. Clearly, $0 \subset \operatorname{dom}\left(t_{n}\right)$ for every $n \in \omega$, so $N_{0}=0$ does the job. Now, suppose there is an $N_{k} \in \omega$ such that $k \subset \operatorname{dom}\left(t_{n}\right)$ for every $n \geq N_{k}$. Suppose the result is not true for $k+1$. Then there is a subsequence $\left\langle t_{n_{m}}: m \in \omega\right\rangle$ of the sequence $\left\langle t_{n}: n \geq N_{k}\right\rangle$ with $\operatorname{dom}\left(t_{n_{m}}\right)<k+1$ for every $m \in \omega$. In this case, note that there is an $l_{0} \in \omega$ such that $t_{n_{m}}(0)=t_{n_{l_{0}}}(0)$ for every $m \geq l_{0}$ (because $\left\langle t_{n_{m}}(0): m \in \omega\right\rangle$ is a decreasing sequence and $<$ well orders $\tau^{*}$ ). With the same argument we find an $l_{1}>l_{0}$ such that $t_{n_{m}}(1)=t_{n_{l_{1}}}(1)$ for every $m \geq l_{1}$. By proceeding in this manner we conclude that there is an $l_{k} \in \omega$ such that $t_{n_{m}}=t_{n_{l_{k}}}$ for every $m \geq l_{k}$, which contradicts the fact that $\left\langle t_{n}: n \in \omega\right\rangle$ is a strictly decreasing sequence.

CLAIM 2.4.4. If $\left\langle t_{n}: n \in \omega\right\rangle$ is a strictly decreasing sequence with respect to $\prec$, then for every $k \in \omega$ there is an $n_{k} \in \omega$ such that $t_{n}(k)=t_{n_{k}}(k)$ for every $n \geq n_{k}$.

Proof. By the previous claim, we may assume (without loss of generality) that $\left\langle t_{n}: n \in \omega\right\rangle$ is such that $\operatorname{dom}\left(t_{n}\right) \subset \operatorname{dom}\left(t_{n+1}\right)$ for every $n \in \omega$. In this case, note that there is an $n_{0} \in \omega$ such that $t_{n}(0)=t_{n_{0}}(0)$ for every $n \geq n_{0}$ (because $\left\langle t_{n}(0): n \in \omega\right\rangle$ is a decreasing sequence and $<$ well orders $\left.\tau^{*}\right)$. Suppose for each $i \leq k$ we have found an $n_{i} \in \omega$ such that $t_{n}(i)=t_{n_{i}}(i)$ for every $n \geq n_{i}$. Then again, because $\left\langle t_{n}(k+1): n \geq n_{k}\right\rangle$ is a decreasing sequence and $<$ well orders $\tau^{*}$, we may find an $n_{k+1}>n_{k}$ such that $t_{n}(k+1)=t_{n_{k+1}}(k+1)$ for every $n \geq n_{k+1}$, which concludes the induction.

Claim 2.4.5. For every $V \in \tau^{*}$ such that $X \neq V, \prec$ is a well order when restricted to $P(V)$.

Proof. Suppose not. Then there is a strictly decreasing sequence $\left\langle t_{n}: n \in \omega\right\rangle$ of elements of $P(V)$. In this case, by the previous claim, for every $k \in \omega$ there is an $n_{k} \in \omega$ such that $t_{n}(k)=t_{n_{k}}(k)$ for every $n \geq n_{k}$. Let $R=\left\langle t_{n_{k}}(k): k \in \omega\right\rangle$. Then $R$ is clearly a run compatible with $\gamma$, so that $\bigcup_{k \in \omega} R(k)=X$. But, since $R(k)=t_{n_{k}}(k) \subset V$ for every $k \in \omega$, we infer that $V=X$.

We define a strategy $\gamma^{*}$ as follows:

- First, let $\gamma^{*}(\langle\rangle)=\gamma(\langle\rangle)$;

- Then, for each $V \in \tau^{*}$ such that $\gamma^{*}(\langle\rangle) \in V$ and $V \neq X$, let $t_{V}=\min P(V)$, with $\min P(V)$ taken with respect to $\prec$ and then set $\gamma^{*}(\langle V\rangle)=\gamma\left(t_{V}\right)$. If $V=X$, let $\gamma^{*}(\langle X\rangle)$ be any fixed point $x \in X$ (the point does not matter, since ALICE has already won the run if BoB plays $X)$.

- Suppose we have defined $\gamma^{*}\left(t\lceil k)\right.$ for every $k \leq|t|$, with $t \in \operatorname{dom}(\gamma)$. Then we let $\gamma^{*}\left(t^{\frown} V\right)=$ $\gamma^{*}(\langle V\rangle)$. 
Clearly, $\gamma^{*}$ satisfies (2.1). To see that it is a winning strategy, let $\left\langle V_{n}: n \in \omega\right\rangle$ be a run compatible with $\gamma^{*}$ (we are omitting ALICE's moves in the run) and set $t_{n}=\min P\left(V_{n}\right)$ (we are assuming that $V_{n} \neq X$ for all $n \in \omega$ since, otherwise, it is already clear that ALICE wins the run). Note that, for each $n \in \omega$, since $t_{n}{ }^{\urcorner} V_{n+1} \in P\left(V_{n+1}\right)$,

$$
t_{n+1} \preceq t_{n} V_{n+1} \cdot
$$

On the other hand, $t_{n}{ }^{\Upsilon} V_{n+1} \prec t_{n}$, so $t_{n+1} \prec t_{n}$ for every $n \in \omega$. By an argument similar to the one presented in the proof of Claim 2.4.5, it follows that for each $k \in \omega$ there is an $n_{k} \in \omega$ such that $t_{n_{k}}(k)=t_{n}(k)$ for every $n \geq n_{k}$. Let $R=\left\langle t_{n_{k}}(k): k \in \omega\right\rangle$. Then $R$ is a run compatible with $\gamma$ and, therefore, $\bigcup_{k \in \omega} R(k)=X$. Since $R(k)=t_{n_{k}}(k) \subset V_{n_{k}}$, we infer that $\bigcup_{n \in \omega} V_{n}=X$, which concludes the proof.

Before showing that a result analogous to Theorem 2.4.2 does not hold for the point-open game, we take a small side track to show that, as it turns out, Theorem 2.3.3 can be generalized to a variety of games. Striving for such generalization, let us first define some new concepts.

Definition 2.4.6. A game $\mathrm{G}=\langle T, A\rangle$ is said to be a positional game for ALICE if given BoB's turns $s, t \in T$ and $x \in \mathrm{B}(\mathrm{G})$ such that $s^{\frown} x, t^{\frown} x \in T$, then $s^{\frown} x^{\frown} y \in T$ if, and only if, $t^{\frown} x^{\frown} y \in T$.

We define a positional game for ВОВ analogously. Then if a game is positional for both players, we simply say it is positional.

The idea behind a positional game for one of the players is that of a game which the restrictions for such player's moves in a given moment does not depend on the entire history of the game so far, only in the current position. Note that the increasing point-open game is, for instance, a positional game just for ALICE, while the point-open game and the finite-open game are positional games for both players (indeed, most of the topological games we are going to study are positional for one of the players, if not both of them). With that being said, we may now introduce a stronger notion of strategy.

Definition 2.4.7. Let $\mathrm{G}=\langle T, A\rangle$ be a positional game for ALICE. We say a strategy $\gamma$ for ALICE is a positional strategy if there is a function $f: \mathrm{B}(\mathrm{G}) \rightarrow \mathrm{A}(\mathrm{G})$ such that, for every $s \in \operatorname{dom}(\gamma)$ and $x \in \mathrm{B}(X)$ with $s^{\wedge} x \in \operatorname{dom}(\gamma)$,

$$
\gamma\left(s^{\wedge} x\right)=f(x)
$$

We define positional strategies for B ОВ analogously.

Intuitively speaking, a strategy for one of the players is positional if it does not depend on the entire history of the game so far, only on the last moment (the current position of the game). Note that Theorem 2.4.2 presents a positional strategy for ALICE in the increasing point-open game. We then present the following generalization of Theorem 2.4.2 (which is a generalization of Theorem 1 from [Galvin and Telgársky 1986]), with the proof also obtained as a generalization of the former's proof . 
Theorem 2.4.8. Suppose $M$ is a set and $T \subset{ }^{<\omega} M$ and $A \subset{ }^{\omega} M$ are such that $\mathrm{G}=\langle T, A\rangle$ is a game. If $\mathrm{G}$ is a positional game for ALICE and there is a preorder $\ll$ over $\mathrm{B}(\mathrm{G})$ such that

(a) for every ALICE's turn $t=\left\langle x_{0}, y_{0}, \ldots, x_{n}, y_{n}\right\rangle$ and $x$ being one of ALICE's possible response to $\left.t, t^{\frown} x\right\urcorner y \in T$ implies that $y \ll y_{n}$;

(b) if $t=\left\langle x_{0}, y_{0}\right\rangle \in T$ and $y \ll y_{0}$, then $\left\langle x_{0}, y\right\rangle \in T$;

(c) if $R, S \in{ }^{\omega} M$ are such that $R \in A$ and for every $n \in \omega$ there is a $k_{n} \in \omega$ such that $S\left(2 k_{n}+\right.$ $1) \ll R(2 n+1)$, then $S \in A$,

then ALICE has a winning strategy in $\mathrm{G}$ if, and only if, ALICE has a positional winning strategy in $\mathrm{G}$.

Analogously, If $\mathrm{G}$ is a positional game for $\mathrm{B} \mathrm{BB}$ and there is a preorder $\ll$ over $\mathrm{A}(\mathrm{G})$ such that

(c) for every Bов's turn $t=\left\langle x_{0}, y_{0}, \ldots, x_{n}\right\rangle$ and y being one of BоB 's possible response to $t$, $\left.t^{\frown} y\right\urcorner x \in T$ implies that $x \ll x_{n}$;

(d) if $t=\left\langle x_{0}\right\rangle \in T$ and $x \ll x_{0}$, then $\langle x\rangle \in T$;

(e) if $R, S \in{ }^{\omega} M$ are such that $R \notin A$ and for every $n \in \omega$ there is a $k_{n} \in \omega$ such that $S\left(2 k_{n}\right) \ll$ $R(2 n)$, then $S \notin A$,

then Вов has a winning strategy in $\mathrm{G}$ if, and only if, Вов has a positional winning strategy in $\mathrm{G}$.

Proof. We will show the result just for AliCE, leaving the case for BOB as a simple execise of adapting the proof. Then let $\gamma$ be a winning strategy for ALICE in G. Let $P=\operatorname{dom}(\gamma)$ and, given $x \in \mathrm{B}(\mathrm{G})$, let $P(x)=\left\{t=\left\langle x_{k}: k \leq n\right\rangle \in P: x_{n}=x\right\}$. Let $<$ be a fixed (strict) well order over B(G), so that $<$ is extended to the following strict linear order over $P: s \prec t$ if either $t \subset s$ or else there is a $k \in \operatorname{dom}(t) \cap \operatorname{dom}(t)$ such that $s\lceil k=t\lceil k$ and $s(k+1)<t(k+1)$.

CLAIM 2.4.9. If $\left\langle t_{n}: n \in \omega\right\rangle$ is a strictly decreasing sequence with respect to $\prec$, then for every $k \in \omega$ there is an $N_{k} \in \omega$ such that $k \subset \operatorname{dom}\left(t_{n}\right)$ for every $n \geq N_{k}$.

Proof. We will prove this by induction. Clearly, $0 \subset \operatorname{dom}\left(t_{n}\right)$ for every $n \in \omega$, so $N_{0}=0$ does the job. Now, suppose there is an $N_{k} \in \omega$ such that $k \subset \operatorname{dom}\left(t_{n}\right)$ for every $n \geq N_{k}$. Suppose the result is not true for $k+1$. Then there is a subsequence $\left\langle t_{n_{m}}: m \in \omega\right\rangle$ of the sequence $\left\langle t_{n}: n \geq N_{k}\right\rangle$ with $\operatorname{dom}\left(t_{n_{m}}\right)<k+1$ for every $m \in \omega$. In this case, note that there is an $l_{0} \in \omega$ such that $t_{n_{m}}(0)=t_{n_{l_{0}}}(0)$ for every $m \geq l_{0}$ (because $\left\langle t_{n_{m}}(0): m \in \omega\right\rangle$ is a decreasing sequence and $<$ well orders $\mathrm{B}(\mathrm{G})$ ). With the same argument we find a $l_{1}>l_{0}$ such that $t_{n_{m}}(1)=t_{n_{l_{1}}}(1)$ for every $m \geq l_{1}$. By proceeding in this manner we conclude that there is an $l_{k} \in \omega$ such that $t_{n_{m}}=t_{n_{l_{k}}}$ for every $m \geq l_{k}$, which contradicts the fact that $\left\langle t_{n}: n \in \omega\right\rangle$ is a strictly decreasing sequence. 
CLAIM 2.4.10. If $\left\langle t_{n}: n \in \omega\right\rangle$ is a strictly decreasing sequence with respect to $\prec$, then for every $k \in \omega$ there is an $n_{k} \in \omega$ such that $t_{n}(k)=t_{n_{k}}(k)$ for every $n \geq n_{k}$.

Proof. By the previous claim, we may assume (without loss of generality) that $\left\langle t_{n}: n \in \omega\right\rangle$ is such that $\operatorname{dom}\left(t_{n}\right) \subset \operatorname{dom}\left(t_{n+1}\right)$ for every $n \in \omega$. In this case, note that there is an $n_{0} \in \omega$ such that $t_{n}(0)=t_{n_{0}}(0)$ for every $n \geq n_{0}$ (because $\left\langle t_{n}(0): n \in \omega\right\rangle$ is a decreasing sequence and $<$ well orders $\mathrm{B}(\mathrm{G})$ ). Suppose that for each $i \leq k$ we have found an $n_{i} \in \omega$ such that $t_{n}(i)=t_{n_{i}}(i)$ for every $n \geq n_{i}$. Then again, because $\left\langle t_{n}(k+1): n \geq n_{k}\right\rangle$ is a decreasing sequence and $<$ well orders $\mathrm{B}(\mathrm{G})$, we find a $n_{k+1}>n_{k}$ such that $t_{n}(k+1)=t_{n_{k+1}}(k+1)$ for every $n \geq n_{k+1}$, which concludes the induction and, therefore, the proof.

Claim 2.4.11. For every $x \in \mathrm{B}(\mathrm{G})$ such that $\langle x, x, x, \ldots\rangle \notin A$, $\prec$ is a well order when restricted to $P(x)$.

Proof. Suppose $\prec$ is not a well order when restricted to $P(x)$. Then there is a strictly decreasing sequence $\left\langle t_{n}: n \in \omega\right\rangle$ of elements of $P(x)$. In this case, by the previous claim, for every $k \in \omega$ there is an $n_{k} \in \omega$ such that $t_{n}(k)=t_{n_{k}}(k)$ for every $n \geq n_{k}$. Let $R=\left\langle t_{n_{k}}(k): k \in \omega\right\rangle$. Then $R$ is clearly a run compatible with $\gamma$, so that $R \in A$. But, since $R(k)=t_{n_{k}}(k) \gg x$ for every $k \in \omega$ (because of condition (a)), we infer by condition (c) that $\langle x, x, x, \ldots\rangle \in A$.

We define $f: \mathrm{B}(\mathrm{G}) \rightarrow \mathrm{A}(\mathrm{G})$ and a winning strategy $\gamma^{*}$ as follows:

- First, let $\gamma^{*}(\langle\rangle)=\gamma(\langle\rangle)$ and for each $x \in \mathrm{B}(X)$ such that $\langle x\rangle \notin \operatorname{dom}(\gamma)$, set $f(x)=a$ for every $a \in \mathrm{A}(x)$;

- Then, for $x \in \mathrm{B}(X)$ such that $\langle x\rangle \in \operatorname{dom}(\gamma)$ and $\langle x, x, x, \ldots\rangle \in A$, let $\gamma^{*}(\langle x\rangle)$ and $f(x)$ be again every fixed $a \in \mathrm{A}(X)$ (it does not matter which, since the game has already been won by ALICE because of conditions (a) and (c)). On the other hand, for each $x \in \mathrm{B}(X)$ such that $\langle x\rangle \in \operatorname{dom}(\gamma)$ and $\langle x, x, x, \ldots\rangle \notin A$, let $t_{x}=\min P(x)$, with $\min P(x)$ taken with respect to $\prec$ and then set $\gamma^{*}(\langle x\rangle)=\gamma\left(t_{x}\right)$.

- Suppose we have defined $\gamma^{*}(t\lceil k)$ for every $k \in \operatorname{dom}(t)$, with $t \in \operatorname{dom}(\gamma)$. Then we let $\gamma^{*}\left(t^{\frown} x\right)=\gamma^{*}(\langle x\rangle)$ (this is well defined in view of the conditions (a) and (b)).

Clearly, $f$ attests that $\gamma^{*}$ is a positional strategy. To see that it is a winning one, let $\left\langle x_{n}: n \in \omega\right\rangle$ be a run compatible with $\gamma^{*}$ (we are omitting ALICE's moves in the run) and set $t_{n}=\min P\left(x_{n}\right)$ (we are assuming that $\left\langle x_{n}, x_{n}, x_{n}, \ldots\right\rangle \notin A$ for all $n \in \omega$ since otherwise, as already remarked, it is clear that ALICE wins the run). Note that, for each $n \in \omega$, since $t_{n}{ }^{\imath} x_{n+1} \in P\left(x_{n+1}\right)$,

$$
t_{n+1} \preceq t_{n} x_{n+1}
$$

On the other hand, $t_{n} x_{n+1} \prec t_{n}$, so $t_{n+1} \prec t_{n}$ for every $n \in \omega$. By an argument similar to the one presented in the proof of Claim 2.4.11, it follows that for each $k \in \omega$ there is an $n_{k} \in \omega$ such that 
$t_{n_{k}}(k)=t_{n}(k)$ for every $n \geq n_{k}$. Let $R=\left\langle t_{n_{k}}(k): k \in \omega\right\rangle$. Then $R$ is a run compatible with $\gamma$ and, therefore, $R \in A$. Since $R(k)=t_{n_{k}}(k) \gg x_{n_{k}}$, we infer that $\left\langle x_{n}: n \in \omega\right\rangle \in A$, which concludes the proof.

Theorem 2.4.8 is indeed a generalization of Theorem 2.4.2: to see this, one just needs to consider the following order over $\tau \backslash\{\emptyset\}$ :

$$
U \ll V \Longleftrightarrow U \supset V
$$

so that Theorem 2.4.2 becomes a corollary of Theorem 2.4.8.

Example 2.4.12. Finally, let us show that even when ALICE has a winning strategy in the pointopen game she may not have a positional winning strategy (which will show the importance of conditions (a), (b) and (c) in Theorem 2.4.8, since the point open game is positional). Consider $5=\{0,1,2,3,4\}$ with the discrete topology. Clearly, ALICE has a winning strategy in the point-open game on 5 (the space is finite, after all).

Now, suppose $\gamma$ is a positional strategy for ALICE in the point-open game on 5. Then note that for every $t \in \operatorname{dom}(\gamma)$ and $V$ open such that $\gamma(\langle\rangle), \gamma(t) \in V$,

$$
\gamma\left(t^{\urcorner} V\right)=\gamma(\langle V\rangle)
$$

Fix sets $A, B \subset 5$ with three points each such that $A \cap B=\{\gamma(\langle\rangle)\}$ (hence, $A \cup B=5$ ) and note that we may assume that, in order to show that $\gamma$ is not a winning strategy, we may assume that $\gamma(\langle A\rangle) \notin A$ and $\gamma(\langle B\rangle) \notin B$ (since it is a positional strategy). Set $C=\{\gamma(\langle A\rangle), \gamma(\langle B\rangle)\}$.

If $\gamma(\langle C\rangle) \in A$, then note that $\gamma$ loses the run

$$
\langle\gamma(\langle\rangle), A, \gamma(\langle A\rangle), C, \gamma(\langle C\rangle), A, \gamma(\langle A\rangle), C, \ldots\rangle,
$$

since $b \in B \backslash\{\gamma(\langle\rangle), \gamma(\langle B\rangle)\}$ will not be covered. If, otherwise, $\gamma(\langle C\rangle) \in B$, then $\gamma$ loses the run

$$
\langle\gamma(\langle\rangle), B, \gamma(\langle B\rangle), C, \gamma(\langle C\rangle), B, \gamma(\langle B\rangle), C, \ldots\rangle,
$$

because, again, $a \in B \backslash\{\gamma(\langle\rangle), \gamma(\langle A\rangle)\}$ will not be covered. It follows that $\gamma$ is not a winning strategy.

Note, however, that ALICE has a positional winning strategy in the finite-open game on 5 (she can pick the whole space right from the start).

Exercise 2.4.13. Show that ALICE has a positional winning strategy in the point-open game on the discrete space $4=\{0,1,2,3\}$.

\subsection{The Banach-Mazur game}

Stefan Banach presented in Problem 43 of the famous "Scottish Book" a game related to the Baire Category Theorem that was proposed by Stanislaw Mazur. Banach himself gave the 
solution for this problem in 1935 - which is why the game became known as the Banach-Mazur game, the first topological game.

Although initially defined on the real line, this game was later generalized on every space, and it goes as follows.

Definition 2.5.1. Given a space $X$, we call Banach-Mazur game, denoted by $\operatorname{BM}(\boldsymbol{X})$, the following game.

- At first, ALICE chooses a non-empty open $U_{0} \subset X$, then B OB chooses a non-empty open $V_{0} \subset U_{0}$;

- In each inning $n \in \omega$ ALICE chooses a non-empty open $U_{n} \subset V_{n-1}$ and BoB chooses a non-empty open $V_{n} \subset U_{n}$.

We then say Bов wins if $\bigcap_{n \in \omega} V_{n} \neq \emptyset$ and AlicE wins otherwise.

Evidently, Вов wins a run $\left\langle U_{0}, V_{0}, \ldots, U_{n}, V_{n}, \ldots\right\rangle$ in $\mathrm{BM}(X)$ if, and only if, $\bigcap_{n \in \omega} U_{n} \neq \emptyset$. In order to help us better understand the game, let us now look at how it behaves on some specific spaces.

Example 2.5.2. If $X$ is a complete metric space, then Bов has a winning strategy in $\operatorname{BM}(X)$. Indeed, consider the strategy $\sigma$ for $\operatorname{BoB}$ in $\operatorname{BM}(X)$ defined as follows:

- In the first inning, if ALICE chooses $U_{0}$, let $x_{0} \in U_{0}$ and $r_{0}<1$ be such that $\bar{B}_{r_{0}}\left(x_{0}\right) \subset U_{0}$ (with $\bar{B}_{r}(x)=\{y \in X: d(x, y) \leq r\}$ ) and set $\sigma\left(\left\langle U_{0}\right\rangle\right)=V_{0}=B_{r_{0}}\left(x_{0}\right)$;

- In general (that is, in the inning $n \in \omega$ ), if ALICE plays with $U_{n}$, let $x_{n} \in U_{n}$ and $r_{n}<\frac{1}{n+1}$ be such that $\bar{B}_{r_{n}}\left(x_{n}\right) \subset U_{n}$ and set $\sigma\left(\left\langle U_{i}: i \leq n\right\rangle\right)=V_{n}=B_{r_{n}}\left(x_{n}\right)$.

Since $r_{n} \rightarrow 0,\left\langle x_{n}: n \in \omega\right\rangle$ is a Cauchy sequence and, therefore, converges to some $x \in X$. Suppose $x \notin V_{N}$ for some $N \in \omega$. Then $x \notin \bar{B}_{r_{N+1}}\left(x_{N+1}\right)$, which contradicts the fact that $x_{n} \rightarrow x$. Hence, $\sigma$ is a winning strategy.

Example 2.5.3. If $X$ is the set of irrational numbers with its usual topology, then $\operatorname{BoB} \uparrow \operatorname{BM}(X)$.

Indeed, fix an enumeration $\left\{q_{n}: n \in \omega\right\}=\mathbb{Q}$. We then define a winning strategy $\sigma$ for Bов in $\operatorname{BM}(X)$ as follows:

- If, in the first inning, ALICE chooses $U_{0}=A_{0} \cap X$, with $A_{0}$ open in $\mathbb{R}$, let $B_{0}=B_{r_{0}}\left(x_{0}\right) \cap X$, with $r_{0}$ and $x_{0}$ picked in a way that $r_{0}<1, q_{0} \notin \bar{B}_{r_{0}}\left(x_{0}\right)$ and $\bar{B}_{r_{0}}\left(x_{0}\right) \subset A_{0}$ and set $\sigma\left(\left\langle U_{0}\right\rangle\right)=$ $V_{0}=B_{0} \cap X$.

- In general (that is, in the inning $n \in \omega$ ), if ALICE chooses $U_{n}=A_{n} \cap X$, with $A_{n}$ open in $\mathbb{R}$, let $B_{n}=B_{r_{n}}\left(x_{n}\right) \cap X$, with $r_{n}$ and $x_{n}$ picked in a way that $r_{n}<\frac{1}{n+1}, q_{n} \notin \bar{B}_{r_{n}}\left(x_{n}\right)$ and $\bar{B}_{r_{n}}\left(x_{n}\right) \subset A_{n}$ and set $\sigma\left(\left\langle U_{i}: i \leq n\right\rangle\right)=V_{n}=B_{n} \cap X$. 
By playing with $\sigma$, ВОВ constructs a Cauchy sequence that cannot converge to any rational number, so it follows from the same argument presented in Example 2.5.2 that $\sigma$ is a winning strategy.

Note that we can repeat the arguments presented in Example 2.5.3 (using Example 2.5.2) to prove the following result.

Proposition 2.5.4. If $X$ is homeomorphic to a dense $G_{\delta}$ subspace of a completely metrizable space, then $\mathrm{B}$ ов $\uparrow \mathrm{BM}(X)$.

Example 2.5.5. If $\mathbb{Q}$ is equipped with its usual topology, then $\operatorname{ALICE} \uparrow B M(\mathbb{Q})$. Indeed, after fixing an enumeration $\mathbb{Q}=\left\{q_{n}: n \in \omega\right\}$, consider the following strategy $\gamma$ for ALICE: in the inning $n \in \omega$, if Вов played with $\left\langle V_{i}: i<n\right\rangle$ thus far, let $\gamma\left(\left\langle V_{i}: i<n\right\rangle\right)=V_{n-1} \backslash\left\{q_{n}\right\}$. By using $\gamma$, ALICE then excludes the entire space throughout a run - so $\gamma$ is a winning strategy.

Note that we can generalize Example 2.5.5 with the same arguments:

Proposition 2.5.6. If $X$ is a countable $T_{1}$ space with an open set $V$ with no isolated points, then $\operatorname{AlicE} \uparrow \operatorname{BM}(X)$.

Example 2.5.7. Let $\mathbb{R}_{l}$ be $\mathbb{R}$ with the lower limit topology (that is, the topology generated by the basis $\mathscr{B}=\left\{\left[a, b[: a, b \in \mathbb{R}\}\right.\right.$, also known as the Sorgenfrey line). Then $\mathrm{Boв} \uparrow \mathrm{BM}\left(\mathbb{R}_{l}\right)$. Indeed, let $\sigma$ be a winning strategy for Вов in $\mathrm{BM}(\mathbb{R})$ and consider the following strategy $\sigma_{l}$ for Вов in $\mathrm{BM}\left(\mathbb{R}_{l}\right)$ (without loss of generality, we assume ALICE will play only with basic open sets):

- In the first inning, if ALICE chooses $A_{0}=\left[a_{0}, b_{0}\left[\right.\right.$, let $\left.A_{0}^{\prime}=\right] a_{0}, b_{0}\left[\right.$ and then set $\sigma_{l}\left(\left\langle A_{0}\right\rangle\right)=$ $\sigma\left(\left\langle A_{0}^{\prime}\right\rangle\right)$

- In general (that is, in the inning $n \in \omega$ ), if ALICE chooses $A_{n}=\left[a_{n}, b_{n}\left[\right.\right.$, let $\left.A_{n}^{\prime}=\right] a_{n}, b_{n}[$ and then set $\sigma_{l}\left(\left\langle A_{i}: i \leq n\right\rangle\right)=\sigma\left(\left\langle A_{i}^{\prime}: i \leq n\right\rangle\right)$.

Clearly, $\sigma_{l}$ is a winning strategy.

Note that the Example 2.5.7 can be generalized with the same arguments to the following result.

Proposition 2.5.8. Let $\langle X, \tau\rangle$ be a space in which $\mathrm{BoB} \uparrow \mathrm{BM}(\langle X, \tau\rangle)$. If $\rho$ is a topology over $X$ such that for every nonempty $V \in \rho$ there is a nonempty $U \in \tau$ with $U \subset V$, then $\operatorname{BoB} \uparrow \operatorname{BM}(\langle X, \rho\rangle)$.

Example 2.5.9. If $K$ is a compact Hausdorff space, then Вов $\uparrow \mathrm{BM}(X)$.

Indeed, consider the following strategy for Вов:

- If AliCE chooses $A_{0}$ in the first inning, ВОВ can use the regularity of the space to find an open set $B_{0}$ such that $\overline{B_{0}} \subset A_{0}$; 
- In general, if ALICE chooses $A_{n}$ in the inning $n \in \omega$, В ов can again find an open set $B_{n}$ such that $\overline{B_{n}} \subset A_{n}$.

Since $\left\{\overline{B_{n}}: n \in \omega\right\}$ has the finite intersection property, there must be an $x \in \bigcap_{n \in \omega} \overline{B_{n}}$. But then, clearly, $x \in \bigcap_{n \in \omega} B_{n}$.

But is the Banach-Mazur game determined on every space? The following theorem will help us find a subspace of the real line showing that this is not the case.

Theorem 2.5.10 (Oxtoby). If Xis a metric space with no isolated points such that $\mathrm{BOB} \uparrow \mathrm{BM}(X)$, then there is a subspace $C \subset X$ such that $C$ is homeomorphic to $2^{\omega}$.

Proof. Let $\sigma$ be winning strategy for $\operatorname{BoB}$ in $\operatorname{BM}(X)$. For each $s \in<\omega_{2}$ we will associate an $r_{s} \in \mathbb{R}$ and an auxiliary point $x_{s}$ such that, for every $s \in{ }^{<\omega_{2}}$ and $i \in 2$,

(a) $r_{s} \leq \frac{1}{2^{|s|}}$

(b) $B_{\left.r_{s}\right\urcorner i}\left(x_{s\urcorner i}\right) \subset B_{r_{s}}\left(x_{s}\right)$;

(c) $B_{r_{s\urcorner 0}}\left(x_{s\urcorner 0}\right) \cap B_{r_{s\urcorner 1}}\left(x_{s\urcorner 1}\right)=\emptyset$;

(d) $\left\langle B_{r_{s \uparrow k}}\left(x_{s \mid k}\right): k \leq|s|\right\rangle \in \operatorname{dom} \sigma$,

using the following recursion.

First, fix any $x_{\langle\rangle} \in X$ and let $r_{s}=1$. Now, suppose we defined $r_{t}$ and $x_{t}$ for every $t \in{ }^{<\omega_{2}}$ such that $|t| \leq n$ for some $n \in \omega$. Then, for each $s \in{ }^{<\omega_{2}}$ such that $|s|=n$, we fix two different points $x_{s^{\urcorner} 0}, x_{s^{\urcorner} 1} \in B_{r_{s}}\left(x_{s}\right)$ (that exist, since $X$ has no isolated points) and for each $i \in 2$ we choose $r_{s^{\urcorner} i} \in \mathbb{R}$ such that

- $r_{s^{\wedge} i} \leq \frac{1}{2^{|s|+1}}$

- $B_{r_{s\urcorner i}}\left(x_{s^{\urcorner} i}\right) \subset \sigma\left(\left\langle B_{r_{s \uparrow k}}\left(x_{s \uparrow k}\right): k \leq|s|\right\rangle\right)$;

- $B_{r_{s\urcorner 0}}\left(x_{s^{\urcorner} 0}\right) \cap B_{r_{s\urcorner 1}}\left(x_{s^{\wedge} 1}\right)=\emptyset$,

and the recursion is complete. Since $\sigma$ is a winning strategy, $\bigcap_{n \in \omega} B_{r_{b\lceil n}}\left(x_{b\lceil n}\right)$ is nonempty for every $b \in 2^{\omega}$. Moreover, by property (a), it can be easily shown that for every $b \in 2^{\omega}$ there is an $x_{b} \in X$ such that,

$$
\bigcap_{n \in \omega} B_{r_{b \nmid n}}\left(x_{b\lceil n}\right)=\left\{x_{b}\right\},
$$

so the function $f: 2^{\omega} \rightarrow X$ defined as $f(b)=x_{b}$ for each $b \in 2^{\omega}$ is injective. We leave to the reader (see Exercise 2.5.19) to show that $f$ is continuous, which concludes the proof.

The subspace of the real line we are going to consider here is a Bernstein set. Recall: 
Definition 2.5.11. A set $B \subset \mathbb{R}$ is a Bernstein set if both $B \cap F$ and $(\mathbb{R} \backslash B) \cap F$ are nonempty for every uncountable closed $F \subset \mathbb{R}$.

Using the Axiom of Choice one can construct a Bernstein set (see e.g. [Ciesielski 1997]) - so we will be assuming here that it exists. In this case:

Lemma 2.5.12. If $B \subset \mathbb{R}$ is a Bernstein set, then $B$ has no isolated points.

Proof. Let $x \in B$ and $I$ be an open interval containing $x$. In this case, let $a, b \in I$ be such that $a<b<x$. Note that $[a, b]$ is an uncountable closed subset of the real line and $[a, b] \subset I \backslash\{x\}$. It follows that $B \cap I \backslash\{x\} \neq \emptyset$ and, hence, $x$ is not isolated in $B$.

Proposition 2.5.13. If $B \subset \mathbb{R}$ is a Bernstein set, then $\mathrm{BM}(B)$ is undetermined.

Proof. Note that if $\mathrm{Boв} \uparrow \mathrm{BM}(B)$, then, by Theorem 2.5.10 there would be a $K \subset B$ which is homeomorphic to $2^{\omega}$. Note that $K$ in this case would be a compact (hence, closed) uncountable subset of $\mathbb{R}$, contradicting the definition of a Bernstein set - so Bов $\Varangle \operatorname{BM}(B)$.

Now, let $\gamma$ be a strategy for ALICE in $\operatorname{BM}(B)$. Following the steps of Theorem 2.5.10's proof we will associate an open interval $I_{s} \subset \mathbb{R}$ for each $s \in{ }^{<\omega_{2}}$ such that, for every $s \in<\omega_{2}$ and $i \in 2$,

(a) $\operatorname{diam}\left(I_{S}\right) \leq \frac{1}{2^{|s|}}$

(b) $\overline{I_{s\urcorner i}} \subset I_{s}$;

(c) $\overline{I_{s\urcorner 0}} \cap \overline{I_{s\urcorner 1}}=\emptyset$;

(d) $\left\langle I_{s}\lceil k \cap B: k \leq|s|\rangle \in \operatorname{dom} \gamma\right.$.

using the following recursion.

First, fix any open interval $I_{\langle\rangle} \subset \mathbb{R}$ such that $\operatorname{diam}\left(I_{\langle\rangle}\right)<1$ and $I_{\langle\rangle} \cap B \subset \gamma(\langle\rangle)$ (this is possible because $\gamma(\langle\rangle)$ is a nonempty open subset of $B$ ). Now, suppose we defined $I_{s}$ for every $s \in \leq n 2$. Then, for each $s \in{ }^{n} 2$, we fix two open intervals $I_{s^{\wedge} 0}, I_{s^{\wedge} 1}$ with diameter less than $\frac{1}{2^{n+1}}$ such that $\overline{I_{s^{\urcorner} i}} \cap B \subset \gamma\left(\left\langle I_{s \mid k}: k \leq n\right\rangle\right)$ for both $i \in 2$ and $\overline{I_{s^{\urcorner} 0}} \cap \overline{I_{s^{\urcorner} 1}}=\emptyset$ (this is possible since, by Lemma 2.5.12, $B$ has no isolated points) and the recursion is complete.

Note that, considering conditions (a), (b) and (c), $\bigcap_{n \in \omega} I_{b\lceil n}=\{x\}$ for each $b \in 2^{\omega}$ and some $x \in \mathbb{R}$. Let $K=\left\{x \in \mathbb{R}:\{x\}=\bigcap_{n \in \omega} I_{b\lceil n}, b \in 2^{\omega}\right\}$. Then, like in the proof of Theorem 2.5.10, it can easily be shown that the function $f: 2^{\omega} \rightarrow K$ is a homeomorphism, so that $K$ is an uncountable closed subset of the real line. In this case, let $x \in K \cap B$ (that exists, by the definition of a Bernstein set) and $b \in 2^{\omega}$ be such that $\{x\}=\bigcap_{n \in \omega} I_{b\lceil n}$. Then, in view of condition (d), $\left\langle I_{b \nmid n} \cap B: n \in \omega\right\rangle$ is a run compatible with $\gamma$. Hence, $\gamma$ is not a winning strategy. 
We will see later on that the Banach-Mazur game is actually closely related to the property of being a Baire space. But for now, let us just remark that $\operatorname{BM}(X)$ is a positional game and, moreover:

Theorem 2.5.14. Given a space $X, \operatorname{ALICE} \uparrow \operatorname{BM}(X)$ if, and only if, AliCE has a positional winning strategy in $\mathrm{BM}(X)$.

Proof. Consider the following order over $\tau \backslash\{\emptyset\}$ :

$$
U \ll V \Longleftrightarrow U \subset V .
$$

Then the result follows from Theorem 2.4.8.

Exercise 2.5.15. Write the details of the proof of Proposition 2.5.4.

Exercise 2.5.16. Write the details of the proof of Proposition 2.5.6.

Exercise 2.5.17. Show that $\mathbb{Q}$ is not a $G_{\delta}$ subset of $\mathbb{R}$.

Example 2.5.18. Write the details of the proof of Proposition 2.5.8.

Exercise 2.5.19. Show that the function $f: 2^{\omega} \rightarrow X$ defined in the proof of Theorem 2.5.10 is continuous.

Hint: Use that $\mathscr{B}=\left\{V_{s}: s \in{ }^{<\omega} 2\right\}$ with $V_{s}=\left\{b \in 2^{\omega}: b \uparrow|s|=s\right\}$ is a basis for $2^{\omega}$. 



\section{SELECTION PRINCIPLES}

Selection principles have been playing a prominent role in the study of combinatorial properties in spaces, so that they are closely related to a whole class of classical topological games (which we will present in the next chapter). In this chapter we introduce the most common variations of selection principles, delivering the classical Scheepers Diagram by the end.

\subsection{Classes of selection principles}

We begin by defining and presenting basic examples of the typical selection principles.

Definition 3.1.1. Given families $\mathscr{A}, \mathscr{B}$, we say that the $\left(\begin{array}{c}\mathscr{A} \\ \mathscr{B}\end{array}\right)$ property holds if for every $A \in \mathscr{A}$ there is a $B \in \mathscr{B}$ such that $B \subset A$.

Example 3.1.2. Given a space $X$, let

- $\mathscr{O}$ be the family of $X$ 's open covers;

- $\mathscr{L}$ be the family of $X$ 's countable open covers;

- $\mathfrak{F}$ be the family of $X$ 's finite open covers.

Then

- $\left(\begin{array}{l}\mathscr{O} \\ \mathscr{L}\end{array}\right)$ holds if, and only if, $X$ is Lindelöf;

- $\left(\begin{array}{l}\mathscr{O} \\ \mathfrak{F}\end{array}\right)$ holds if, and only if, $X$ is compact.

Property $(\stackrel{\mathscr{B}}{\mathscr{B}})$ is rather simple, as the reader must have certainly come across properties such as the ones presented in Example 3.1.2, probably not referred as selection principles. What may come as new properties are the ones that follow. 
Definition 3.1.3. Given families $\mathscr{A}, \mathscr{B}$, we say that the $S_{1}(\mathscr{A}, \mathscr{B})$ property holds if for every sequence $\left\langle A_{n}: n \in \omega\right\rangle$ of elements of $\mathscr{A}$, there exists a sequence $\left\langle b_{n}: n \in \omega\right\rangle$ such that $b_{n} \in A_{n}$ for every $n \in \omega$ and $\left\{b_{n}: n \in \omega\right\} \in \mathscr{B}$.

Example 3.1.4. If, given a space $X, \mathrm{~S}_{1}(\mathscr{O}, \mathscr{O})$ holds, we say that $X$ is a Rothberger space. In other words, $X$ is a Rothberger space if for every sequence $\left\langle\mathscr{U}_{n}: n \in \omega\right\rangle$ of open covers of $X$ there is a sequence of open sets $\left\langle U_{n}: n \in \omega\right\rangle$ such that $U_{n} \in \mathscr{U}_{n}$ for every $n \in \omega$ and $\bigcup_{n \in \omega} U_{n}=X$.

Definition 3.1.5. Given families $\mathscr{A}, \mathscr{B}$ and $k \geq 2$, we say that the $\mathrm{S}_{\mathrm{k}}(\mathscr{A}, \mathscr{B})$ property holds if for every sequence $\left\langle A_{n}: n \in \omega\right\rangle$ of elements of $\mathscr{A}$, there exists a sequence $\left\langle B_{n}: n \in \omega\right\rangle$ such that each $B_{n}$ is a subset of $A_{n}$ with at most $k$ elements and $\bigcup_{n \in \omega} B_{n} \in \mathscr{B}$.

Definition 3.1.6. Given families $\mathscr{A}, \mathscr{B}$, we say that the $\mathrm{S}_{\text {fin }}(\mathscr{A}, \mathscr{B})$ property holds if for every sequence $\left\langle A_{n}: n \in \omega\right\rangle$ of elements of $\mathscr{A}$, there exists a sequence $\left\langle F_{n}: n \in \omega\right\rangle$ such that each $F_{n}$ is a finite subset of $A_{n}$ and $\bigcup_{n \in \omega} F_{n} \in \mathscr{B}$.

Example 3.1.7. If, given a space $X, \mathrm{~S}_{\mathrm{fin}}(\mathscr{O}, \mathscr{O})$ holds, we say that $X$ is a Menger space. In other words, $X$ is a Menger space if for every sequence $\left\langle\mathscr{U}_{n}: n \in \omega\right\rangle$ of open covers of $X$ there is a sequence of finite sets $\left\langle\mathscr{F}_{n}: n \in \omega\right\rangle$ such that $\mathscr{F}_{n} \subset \mathscr{U}_{n}$ for every $n \in \omega$ and $\bigcup_{n \in \omega} \mathscr{F}_{n}$ is an open cover of $X$.

Right off the bat we may show some trivial general results about these selection principles that usually relate to one another in a simple way.

Proposition 3.1.8. Let $\mathscr{A}$ and $\mathscr{B}$ be families of sets. If $\mathscr{C} \subset \mathscr{A}$ and $\mathscr{D} \subset \mathscr{B}$, then:

- $\left(\begin{array}{c}\mathscr{A} \\ \mathscr{D}\end{array}\right) \Longrightarrow\left(\begin{array}{c}\mathscr{A} \\ \mathscr{B}\end{array}\right) \Longrightarrow\left(\begin{array}{c}\mathscr{C} \\ \mathscr{B}\end{array}\right)$;

- $\mathrm{S}_{1}(\mathscr{A}, \mathscr{D}) \Longrightarrow \mathrm{S}_{1}(\mathscr{A}, \mathscr{B}) \Longrightarrow \mathrm{S}_{1}(\mathscr{C}, \mathscr{B})$;

- for every $k \geq 2, \mathrm{~S}_{\mathrm{k}}(\mathscr{A}, \mathscr{D}) \Longrightarrow \mathrm{S}_{\mathrm{k}}(\mathscr{A}, \mathscr{B}) \Longrightarrow \mathrm{S}_{\mathrm{k}}(\mathscr{C}, \mathscr{B})$;

- $\mathrm{S}_{\text {fin }}(\mathscr{A}, \mathscr{D}) \Longrightarrow \mathrm{S}_{\text {fin }}(\mathscr{A}, \mathscr{B}) \Longrightarrow \mathrm{S}_{\text {fin }}(\mathscr{C}, \mathscr{B})$.

Proposition 3.1.9. Let $\mathscr{A}$ and $\mathscr{B}$ be families of sets. Then

$$
\mathrm{S}_{1}(\mathscr{A}, \mathscr{B}) \Longrightarrow \mathrm{S}_{\mathrm{k}}(\mathscr{A}, \mathscr{B}) \Longrightarrow \mathrm{S}_{\mathrm{fin}}(\mathscr{A}, \mathscr{B}) \Longrightarrow\left(\begin{array}{c}
\mathscr{A} \\
\mathscr{B}
\end{array}\right)
$$

The generality of Definitions 3.1.3, 3.1.5 and 3.1.6 might give rise to many set theoretical combinatorial properties. However, as already emphasized, we are interested here mainly in topology - so, with this in mind, let us study some interesting particular cases.

Exercise 3.1.10. Write the details of Propositions 3.1.8 and 3.1.9's proofs. 


\subsection{Closure properties}

Given a space $X$ and $x \in X$, we denote as $\Omega_{x}$ the family of subsets of $X$ with $x$ in their closure.

Definition 3.2.1. We say a space $X$ has countable fan-tightness at $x \in X$, if $\mathrm{S}_{\text {fin }}\left(\Omega_{x}, \Omega_{x}\right)$ holds, that is, for every sequence $\left\langle A_{n}: n \in \omega\right\rangle$ of subsets of $X$ such that $x \in \overline{A_{n}}$ for every $n \in \omega$, we may pick $B_{n} \subset A_{n}$ finite in a way that $x \in \overline{\bigcup_{n \in \omega} B_{n}}$.

Example 3.2.2. If $X$ has a countable local basis at $x \in X$, then clearly $\mathrm{S}_{\text {fin }}\left(\Omega_{x}, \Omega_{x}\right)$ holds.

Definition 3.2.3. We say a space $X$ has countable strong fan-tightness at $x \in X$ if $\mathrm{S}_{1}\left(\Omega_{x}, \Omega_{x}\right)$ holds, that is, for every sequence $\left\langle A_{n}: n \in \omega\right\rangle$ of subsets of $X$ such that $x \in \overline{A_{n}}$ for every $n \in \omega$, we may pick $b_{n} \in A_{n}$ in a way that $x \in \overline{\left\{b_{n}: n \in \omega\right\}}$.

Example 3.2.4. Clearly, $\mathrm{S}_{1}\left(\Omega_{x}, \Omega_{x}\right)$ also holds in the spaces given in Example 3.2.2.

In view of Examples 3.2.2 and 3.2.4 one may wonder whether there is a space on which $\mathrm{S}_{\text {fin }}\left(\Omega_{x}, \Omega_{x}\right)$ holds, but $\mathrm{S}_{1}\left(\Omega_{x}, \Omega_{x}\right)$ does not hold. Indeed, we gave different names for these two properties for a reason: they are different. We present in Section 5.7 a whole class of examples that attests this assertion. What we can show now is that $S_{1}\left(\Omega_{x}, \Omega_{x}\right)$ is equivalent to $S_{k}\left(\Omega_{x}, \Omega_{x}\right)$ for every $k \geq 2$ :

Proposition 3.2.5 ([García-Ferreira and Tamariz-Mascarúa 1995]). Let $X$ be a space and $x \in X$. Then $\mathrm{S}_{1}\left(\Omega_{x}, \Omega_{x}\right)$ holds if, and only if, $\mathrm{S}_{\mathrm{k}}\left(\Omega_{x}, \Omega_{x}\right)$ holds for every $k \geq 2$.

Proof. It is clear that if $\mathrm{S}_{1}\left(\Omega_{x}, \Omega_{x}\right)$ holds, then $\mathrm{S}_{\mathrm{k}}\left(\Omega_{x}, \Omega_{x}\right)$ holds for every $k \in \mathbb{N}$, so suppose $\mathrm{S}_{\mathrm{k}}\left(\Omega_{x}, \Omega_{x}\right)$ holds for some $k \geq 2$ and let $\left\langle A_{n}: n \in \omega\right\rangle$ be a sequence of subsets of $X$ such that $x \in \overline{A_{n}}$ for every $n \in \omega$. Since $\mathrm{S}_{\mathrm{k}}\left(\Omega_{x}, \Omega_{x}\right)$ holds, there is a sequence $\left\langle B_{n}: n \in \omega\right\rangle$ and $k \in \omega$ with, for every $n \in \omega$,
a. $B_{n} \subset A_{n}$;
b. $x \in \overline{\bigcup_{n \in \omega} B_{n}}$;
c. $\left|B_{n}\right| \leq k$.

Without loss of generality, we assume that $\left|B_{n}\right|=k$ for every $n \in \omega$ and we write $B_{n}=$ $\left\{b_{n}^{1}, \ldots, b_{n}^{k}\right\}$ for each $n \in \omega$. Now, let $C_{i}=\left\{b_{n}^{i}: n \in \omega\right\}$ for each $i \leq k$.

CLAIM 3.2.6. There is an $i \leq k$ such that $x \in \overline{C_{i}}$.

Proof. Suppose not. Then for each $i \leq k$ there is an open set $V_{i}$ with $x \in V_{i}$ and $V_{i} \cap C_{i}=\emptyset$. Then by letting $V=\bigcap_{i \leq k} V_{i}$,

$$
V \cap\left(\bigcup_{n \in \omega} B_{n}\right)=V \cap \bigcup_{i \leq k} C_{i}=\emptyset
$$


which contradicts $b$.

Let $m \leq k$ be such that $x \in \overline{C_{m}}$. Then the sequence $\left\langle b_{n}^{m}: n \in \omega\right\rangle$ attests $\mathrm{S}_{1}\left(\Omega_{x}, \Omega_{x}\right)$ and the proof is complete.

It should be noted that we can analogously show the same result in the context of another closure-related selection property:

Definition 3.2.7. Given a space $X$, we write $\mathrm{D}$ as the collection of all dense subsets of $X$.

Proposition 3.2.8. Let $X$ be a space. Then $\mathrm{S}_{1}(\mathrm{D}, \mathrm{D})$ holds if, and only if, $\mathrm{S}_{\mathrm{k}}(\mathrm{D}, \mathrm{D})$ holds for every $k \geq 2$.

This is all we have to talk about closure-related selection principles for now, but there will be more to discuss about it once we go back to looking at games later on.

Exercise 3.2.9. Write the details of Proposition 3.2.8's proof.

\subsection{Covering properties}

In Examples 3.1.4 and 3.1.7 we have already presented some covering selection principles. In this section we take a further look at these properties, showing how they ultimately relate to one another with the Scheepers Diagram.

\subsubsection{Menger spaces}

Recall that a space $X$ is a Menger space if $\mathrm{S}_{\text {fin }}(\mathscr{O}, \mathscr{O})$ holds, that is, if for each sequence of open covers $\left\langle\mathscr{U}_{n}: n \in \omega\right\rangle$ there is a sequence $\left\langle\mathscr{F}_{n}: n \in \omega\right\rangle$ such that each $\mathscr{F}_{n}$ is a finite subset of $\mathscr{U}_{n}$ and $\bigcup_{n \in \omega} \mathscr{F}_{n}$ is a cover for the space.

Example 3.3.1. Every compact space is a Menger space. Indeed, if $X$ is compact, given a sequence o open covers $\left\langle\mathscr{U}_{n}: n \in \omega\right\rangle$ one may find a finite subcover $\mathscr{F}_{n} \subset \mathscr{U}_{n}$ of $X$ for each $n \in \omega$, which witnesses $S_{\text {fin }}(\mathscr{O}, \mathscr{O})$.

It is easy to see that $\mathrm{S}_{\text {fin }}(\mathscr{O}, \mathscr{O})$ holds if, and only if $\mathrm{S}_{\text {fin }}(\mathscr{O}, \mathscr{L})$ holds, so the following result is a trivial corollary of Proposition 3.1.9.

Proposition 3.3.2. If $\mathrm{S}_{\mathrm{fin}}(\mathscr{O}, \mathscr{O})$ holds on a space $X$, then $X$ is Lindelöf.

The next result, on the other hand, requires a bit more work to show.

Proposition 3.3.3. Let $\left\{X_{n}: n \in \omega\right\}$ be a collection of spaces such that $\mathrm{S}_{\mathrm{fin}}(\mathscr{O}, \mathscr{O})$ holds on $X_{n}$ for every $n \in \omega$. Then $\mathrm{S}_{\text {fin }}(\mathscr{O}, \mathscr{O})$ holds on $Y=\bigcup_{n \in \omega} X_{n}$. 
Proof. Let $\left\langle\mathscr{U}_{n}: n \in \omega\right\rangle$ be a sequence of open covers of $Y$ and fix a partition $\left\{P_{k}: k \in \omega\right\}$ of $\omega$ into infinite sets. For each $k \in \omega$ and $n \in P_{k}$, let $\mathscr{V}_{n}=\left\{V^{\prime}=V \cap X_{k}: V \in \mathscr{U}_{n}\right\}$. Note that, for each $k \in \omega,\left\langle\mathscr{V}_{n}: n \in P_{k}\right\rangle$ is a sequence of open covers of $X_{k}$, so let $\left\langle\mathscr{F}_{n}^{\prime}: n \in P_{k}\right\rangle$ be a sequence witnessing $\mathrm{S}_{\text {fin }}(\mathscr{O}, \mathscr{O})$ on $X_{k}$ for $\left\langle\mathscr{V}_{n}: n \in P_{k}\right\rangle$. In this case, for each $n \in P_{k}$, let $\mathscr{F}_{n} \subset \mathscr{U}_{n}$ be a finite set such that $\left\{V \cap X_{k}: V \in \mathscr{F}_{n}\right\}=\mathscr{F}_{n}^{\prime}$. Then, clearly, $\left\langle\mathscr{F}_{n}: n \in \omega\right\rangle$ witnesses $\mathrm{S}_{\text {fin }}(\mathscr{O}, \mathscr{O})$ on $Y$ for $\left\langle\mathscr{U}_{n}: n \in \omega\right\rangle$, and the proof is complete.

Corollary 3.3.4. If $X$ is a $\sigma$-compact space, then $\mathrm{S}_{\mathrm{fin}}(\mathscr{O}, \mathscr{O})$ holds on $X$. In particular, $\mathrm{S}_{\mathrm{fin}}(\mathscr{O}, \mathscr{O})$ holds on the real line.

Before moving on to the Rothberger property, we show that the Menger property is preserved under continuous images:

Proposition 3.3.5. If $\mathrm{S}_{\text {fin }}(\mathscr{O}, \mathscr{O})$ holds on $X$ and $f: X \rightarrow Y$ is a continuous function, then $\mathrm{S}_{\text {fin }}(\mathscr{O}, \mathscr{O})$ also holds on $f[X]$.

Proof. Let $\left\langle\mathscr{U}_{n}: n \in \omega\right\rangle$ be a sequence of open covers of $f[X]$. Then $\mathscr{V}_{n}=\left\{f^{-1}(U): U \in \mathscr{U}_{n}\right\}$ is an open cover of $X$ for every $n \in \omega$. Since $\mathrm{S}_{\text {fin }}(\mathscr{O}, \mathscr{O})$ holds on $X$, there is a sequence $\left\langle\mathscr{F}_{n}: n \in \omega\right\rangle$ such that each $\mathscr{F}_{n} \subset \mathscr{U}_{n}$ is finite and $\bigcup_{n \in \omega} \bigcup_{U \in \mathscr{F}_{n}} f^{-1}(U)=X$, which implies that $f[X] \subset \bigcup_{n \in \omega} \cup \mathscr{F}_{n}$, and the proof is complete.

\subsubsection{Rothberger spaces}

Recall that a space $X$ is a Rothberger space if $S_{1}(\mathscr{O}, \mathscr{O})$ holds, that is, if for each sequence of open covers $\left\langle\mathscr{U}_{n}: n \in \omega\right\rangle$ there is a sequence $\left\langle U_{n}: n \in \omega\right\rangle$ such that $U_{n} \in \mathscr{U}_{n}$ for every $n \in \omega$ and $\left\{U_{n}: n \in \omega\right\}$ is a cover for the space.

Example 3.3.6. If $X$ is a countable space, then $X$ is clearly a Rothberger space.

One may wonder whether compact spaces are also Rothberger. However, this is not the case:

Proposition 3.3.7. There is a compact space $K$ (namely, the Cantor set $2^{\omega}$ ) such that $\mathrm{S}_{1}(\mathscr{O}, \mathscr{O})$ does not hold on $K$.

Proof. For each $n \in \omega$, consider $\pi_{n}: 2^{\omega} \rightarrow 2$ as the projection of the $n$th coordinate and let $V_{n}^{i}=\pi_{n}^{-1}(i)$ with $i \in 2$. Then $\mathscr{U}_{n}=\left\{V_{n}^{i}: i \in 2\right\}$ is an open cover for $2^{\omega}$ for every $n \in \omega$. Moreover, if for each $n \in \omega$ we choose any $V_{n}^{i_{n}}$ with $i_{n} \in 2$, then the point $\left\langle j_{n}: n \in \omega\right\rangle$, with $j_{n} \neq i_{n}$ for each $n$, will not be covered, which gives us the desired result.

Note that, as a bonus, Proposition 3.3.7 gives us a space on which $\mathrm{S}_{\text {fin }}(\mathscr{O}, \mathscr{O})$ holds, but $\mathrm{S}_{1}(\mathscr{O}, \mathscr{O})$ does not hold - so both properties are quite different.

But how does $S_{1}(\mathscr{O}, \mathscr{O})$ behave in the real line? Well: 
Proposition 3.3.8. If $\mathbb{R}$ is equipped with the usual topology, then $S_{1}(\mathscr{O}, \mathscr{O})$ does not hold on $\mathbb{R}$.

Proof. Consider the sequence of open cover $\left\langle\mathscr{U}_{n}: n \in \omega\right\rangle$ defined by

$$
\mathscr{U}_{n}=\{] x, x+\frac{1}{2^{n}}[: x \in \mathbb{R}\} .
$$

Then, if $U_{n} \in \mathscr{U}_{n}$ is picked for each $n \in \omega$, the Lebesgue measure of $\bigcup_{n \in \omega} U_{n}$ will be bounded by 2 and, therefore, $\mathbb{R} \neq \bigcup_{n \in \omega} U_{n}$.

Proposition 3.3.9. If $X \subset \mathbb{R}$ has $M>0$ as its Lesbegue measure, then $\mathrm{S}_{1}(\mathscr{O}, \mathscr{O})$ does not hold on $X$.

Proof. Inspired by the proof of Proposition 3.3.8, just consider the sequence $\left\langle\mathscr{U}_{n}: n \in \omega\right\rangle$ defined by

$$
\mathscr{U}_{n}=\{] x, x+\frac{M}{2} \frac{1}{2^{n}}[\cap X: x \in \mathbb{R}\}
$$

and the conclusion follows from the same argument as in the proof of Proposition 3.3.8.

In view of Propositions 3.3.8 and 3.3.9 one may ask:

Question 3.3.10. Is there any uncountable subspace of the real line on which $\mathrm{S}_{1}(\mathscr{O}, \mathscr{O})$ holds?

As it turns out, the answer to this question is independent of ZFC - we present here the consistency of the positive answer using a Luzin set (see 1.2.6 for the definition and 1.2.7 for a construction using $\mathrm{CH})$ :

Proposition 3.3.11 ([Rothberger 1938]). If $L \subset \mathbb{R}$ is a Luzin set, then $\mathrm{S}_{1}(\mathscr{O}, \mathscr{O})$ holds on $L$.

Proof. Let $L \subset \mathbb{R}$ be a Luzin set and $D=\left\{d_{k}: k \in \omega\right\} \subset L$ be a countable dense subset (recall that $\mathbb{R}$ is hereditarily separable, since it has a countable basis). Now, if $\left\langle\mathscr{U}_{n}: n \in \omega\right\rangle$ is a sequence of covers of $L$ by open subsets of $\mathbb{R}$, we first pick, for each $k \in \omega, U_{2 k} \in \mathscr{U}_{2 k}$ such that $d_{k} \in U_{2 k}$.

Note that $(\mathbb{R} \backslash \bar{L}) \cup \bigcup_{k \in \omega} U_{2 k}$ is open and dense, so $F=L \backslash \bigcup_{k \in \omega} U_{2 k}$ is nowhere dense, so that $F$ is at most countable. Then we can easily cover $F$ by picking one open set of each one of the remaining covers $\left\{\mathscr{U}_{2 k+1}: k \in \omega\right\}$, which gives us the desired result.

Actually, Question 3.3.10 is closely related to Question 2.3.13. This relation will be clear later, once we go back to talking about topological games. For now, it should be noted that, just like Menger spaces, Rothberger spaces behave well under continuous images:

Proposition 3.3.12. If $\mathrm{S}_{1}(\mathscr{O}, \mathscr{O})$ holds on $X$ and $f: X \rightarrow Y$ is a continuous function, then $\mathrm{S}_{1}(\mathscr{O}, \mathscr{O})$ also holds on $f[X]$. 
Proposition 3.3.12 allows us to point out a necessary condition over regular Rothberger spaces that will later play an important role when we study measure theory (its proof can be found in [Bukovský 2010]):

Theorem 3.3.13. Let $X$ be a regular Rothberger space. Then $X$ is zero-dimensional.

Proof. If $X$ is Rothberger, then $X$ is Lindelöf, so it follows from Theorem 1.2.3 that $X$ is normal (therefore, in particular, $T_{3 \frac{1}{2}}$ ).

Let $x \in X$ and $V$ be an open neighborhood of $x$. Since $X$ is $T_{3 \frac{1}{2}}$, there is a continuous function $f: X \rightarrow[0,1]$ such that $f(x)=0$ and $f(y)=1$ for every $y \in X \backslash V$. By Proposition 3.3.12, $f[X]$ is also Rothberger, so $f[X] \neq[0,1]$ (since, by Proposition 3.3.9, $[0,1]$ is not Rothberger). Let $\varepsilon \in] 0,1\left[\right.$ be such that $f(z) \neq \varepsilon$ for all $z \in X$. Then $f^{-1}\left(\left[0, \varepsilon[)=f^{-1}([0, \varepsilon])\right.\right.$ is a clopen subset of $V$ containing $x$, which concludes the proof.

And finally (for now), the following proposition can be shown proceeding with the steps of Proposition 3.3.3, so it will be left as an exercise (3.3.16).

Proposition 3.3.14. Let $\left\{X_{n}: n \in \omega\right\}$ be a collection of spaces such that $\mathrm{S}_{1}(\mathscr{O}, \mathscr{O})$ holds on $X_{n}$ for every $n \in \omega$. Then $\mathrm{S}_{1}(\mathscr{O}, \mathscr{O})$ holds on $Y=\bigcup_{n \in \omega} X_{n}$.

Exercise 3.3.15. Write the details of Proposition 3.3.12's proof.

Exercise 3.3.16. Write the details of Proposition 3.3.14's proof.

\subsubsection{Hurewicz spaces}

We now take a look at a new kind of covering selection principle that will appear in the Scheepers Diagram. It goes as follows:

Definition 3.3.17. Given a space $X$ and families $\mathscr{A}, \mathscr{B}$ of covers of $X$, we say that the $\mathrm{U}_{\text {fin }}(\mathscr{A}, \mathscr{B})$ property holds if for every sequence $\left\langle\mathscr{U}_{n}: n \in \omega\right\rangle$ of covers from $\mathscr{A}$, there exists a sequence $\left\langle\mathscr{F}_{n}: n \in \omega\right\rangle$ such that each $\mathscr{F}_{n}$ is a finite subset of $\mathscr{U}_{n}$ and either $\bigcup \mathscr{F}_{n}=X$ for some $n \in \omega$, or else $\left\{\bigcup \mathscr{F}_{n}: n \in \omega\right\} \in \mathscr{B}$.

Our main example of a $U_{\text {fin }}(\mathscr{A}, \mathscr{B})$ selection principle is the one that characterizes Hurewicz spaces:

Example 3.3.18. Given a space, let $\Gamma$ denote the subcollection of $\mathscr{O}$ such that $\mathscr{U} \in \Gamma$ if $\mathscr{U}$ is infinite and, for every $x \in X,\{U \in \mathscr{U}: x \notin U\}$ is finite. A cover $\mathscr{U} \in \Gamma$ is called a $\boldsymbol{\gamma}$-cover.

We then say $X$ is a Hurewicz space if $\mathrm{U}_{\text {fin }}(\mathscr{O}, \Gamma)$ holds, that is, if for every sequence of open covers $\left\langle\mathscr{U}_{n}: n \in \omega\right\rangle$ there is a sequence $\left\langle\mathscr{F}_{n}: n \in \omega\right\rangle$ such that either $\bigcup \mathscr{F}_{n}=X$ for some $n \in \omega$, or else $\left\{\bigcup \mathscr{F}_{n}: n \in \omega\right\} \in \Gamma$. 
But before looking further into the Hurewicz space's case, let us first present some trivial general results, which will be left as exercises (see 3.3.28):

Proposition 3.3.19. Let $\mathscr{A}$ and $\mathscr{B}$ be families of covers of a space $X$. If $\mathscr{C} \subset \mathscr{A}$ and $\mathscr{D} \subset \mathscr{B}$, then:

$$
\mathrm{U}_{\text {fin }}(\mathscr{A}, \mathscr{D}) \Longrightarrow \mathrm{U}_{\text {fin }}(\mathscr{A}, \mathscr{B}) \Longrightarrow \mathrm{U}_{\text {fin }}(\mathscr{C}, \mathscr{B})
$$

Proposition 3.3.20. If $\mathscr{A}$ and $\mathscr{B}$ are families of covers of a space $X$ and $\mathrm{S}_{1}(\mathscr{A}, \mathscr{B})$ holds, then $\mathrm{U}_{\text {fin }}(\mathscr{A}, \mathscr{B})$ holds.

Proposition 3.3.21. For every family $\mathscr{A} \subset \mathscr{O}$,

$$
\mathrm{U}_{\text {fin }}(\mathscr{A}, \mathscr{O}) \Longleftrightarrow \mathrm{S}_{\text {fin }}(\mathscr{A}, \mathscr{O})
$$

Now, back to Hurewicz spaces: we continue by presenting some characterizations.

Theorem 3.3.22. A countably infinite open cover $\mathscr{U}$ is a $\gamma$-cover if, and only if, $\mathscr{V}$ is an open cover provided $\mathscr{V} \subset \mathscr{U}$ is infinite.

Proof. If $\mathscr{U}$ is a $\gamma$-cover, then it is clear that every infinite $\mathscr{V} \subset \mathscr{U}$ is an open cover. Now, suppose $\mathscr{U}$ is not a $\gamma$-cover. Then there is an $x \in X$ such that $\mathscr{V}=\{U \in \mathscr{U}: x \notin U\}$ is infinite. In this case, $\mathscr{V}$ is an infinite subset of $\mathscr{U}$ that does not cover $X$, which concludes the proof.

Corollary 3.3.23. A countably infinite open cover $\mathscr{U}$ is a $\gamma$-cover if, and only if, $\mathscr{V}$ is a $\gamma$-cover for every infinite $\mathscr{V} \subset \mathscr{U}$.

Proposition 3.3.24. The following properties are equivalent on every space $X$ :

(a) $\mathrm{U}_{\text {fin }}(\mathscr{O}, \Gamma)$ holds;

(b) for every sequence of open covers $\left\langle\mathscr{U}_{n}: n \in \omega\right\rangle$ of $X$ there is a sequence $\left\langle\mathscr{F}_{n}: n \in \omega\right\rangle$ such that $\mathscr{V}=\left\{\bigcup \mathscr{F}_{n}: n \in \omega\right\} \in \mathscr{O}$ and each $x \in X$ is in all but finitely many open sets of $\mathscr{V}$.

Proof. It is clear that if (b) holds, then $\mathrm{U}_{\text {fin }}(\mathscr{O}, \Gamma)$ holds, so suppose $\mathrm{U}_{\text {fin }}(\mathscr{O}, \Gamma)$ holds and let $\left\langle\mathscr{U}_{n}: n \in \omega\right\rangle$ be a sequence of open covers of $X$. If for all but finitely many $n \in \omega$ there is a finite subcover $\mathscr{F}_{n} \subset \mathscr{U}_{n}$, so we may assume that for every $n \in \omega, \mathscr{U}_{n}$ has no finite subcover. In this case, applying $\mathrm{U}_{\text {fin }}(\mathscr{O}, \Gamma)$ to $\left\langle\mathscr{U}_{n}: n \in \omega\right\rangle$ grants us a sequence $\left\langle\mathscr{F}_{n}: n \in \omega\right\rangle$ such that each $\mathscr{F}_{n}$ is a finite subset of $\mathscr{U}_{n}$ and $\left\{\bigcup \mathscr{F}_{n}: n \in \omega\right\}$ is a $\gamma$-cover, which concludes the proof.

It should be noted that, like Menger and Rothberger spaces, Hurewicz spaces are also Lindelöf:

Proposition 3.3.25. If $\mathrm{U}_{\mathrm{fin}}(\mathscr{O}, \Gamma)$ holds on a space $X$, then $X$ is Lindelöf. 
Proof. Let $\mathscr{U} \in \mathscr{O}$ and set $\left\langle\mathscr{U}_{n}: n \in \omega\right\rangle$ with $\mathscr{U}_{n}=\mathscr{U}$ for every $n \in \omega$. In this case, applying $\mathrm{U}_{\text {fin }}(\mathscr{O}, \Gamma)$ to $\left\langle\mathscr{U}_{n}: n \in \omega\right\rangle$ grants us a sequence $\left\langle\mathscr{F}_{n}: n \in \omega\right\rangle$ of finite subsets of $\mathscr{U}$ such that, in particular, $\bigcup_{n \in \omega} \mathscr{F}_{n} \in \mathscr{O}$, so $X$ is Lindelöf.

Surprisingly, the $U_{\text {fin }}(\mathscr{O}, \Gamma)$ property is closely related to how a space $X$ embeds on its compactification. We dedicate the rest of this subsection to show this relation, starting with the following concept.

Definition 3.3.26. We say a completely regular space $X$ is Čech-complete if $X$ is a $G_{\delta}$ set in its Stone-Čech compactification $\beta X$.

It should be noted that $X$ is Čech-complete if, and only if, $X$ is a $G_{\delta}$ set in every compact Hausdorff space $K \supset X$.

Theorem 3.3.27. A completely regular space $X$ is Hurewicz if, and only if, for every $\check{C}$ echcomplete space $G \supset X$ there is a $\sigma$-compact $F$ such that $X \subset F \subset G$.

Proof. Suppose $X$ is Hurewicz. If $X$ is compact, then the desired implication is trivial, so we assume $X$ is not compact and let $G \supset X$ be such that $G=\bigcap_{n \in \omega} A_{n}$, with $A_{n}$ open in $\beta G$ (without loss of generality, we will assume that $A_{n+1} \subset A_{n}$ for all $\left.n \in \omega\right)$. Using regularity and noncompacity, choose an open set $U_{x}^{n}$ for each $x \in X$ such that $x \in U_{x}^{n} \subset \overline{U_{x}^{n}} \subset A_{n}$ and $\left\{U_{x}^{n}: x \in X\right\}$ has no finite subcover for all $n \in \omega$. Since $X$ is Hurewicz, it is also Lindelöf, so for each $n \in \omega$ there is a countable set $\left\{x_{j}^{n} \in X: j \in \omega\right\}$ such that $\left\{U_{x_{j}^{n}}^{n}: j \in \omega\right\}$ is an open cover. Now, if we let $U_{k}^{n}=\bigcup_{j \leq k} U_{x_{j}^{n}}^{n}$, then $\overline{U_{k}^{n}} \subset A_{n}$ and $\mathscr{U}_{n}=\left\{U_{k}^{n}: k \in \omega\right\}$ is an increasing open cover for $X$ for every $n \in \omega$. In this case, by applying the Hurewicz property, we can choose a natural number $k_{n}$ for each $n \in \omega$ such that $\mathscr{V}=\left\{U_{k_{n}}^{n}: n \in \omega\right\}$ is an open cover for $X$ such that each $x \in X$ belongs to all but finitely many open sets of $\mathscr{V}$ (note that $U_{k_{n}}^{n} \not \supset X$ for all $n \in \omega$ because we assumed $\left\{U_{x}^{n}: x \in X\right\}$ has no finite subcover for all $\left.n \in \omega\right)$. So it follows that if we let $K_{n}=\bigcap_{m \geq n} \overline{U_{k_{m}}^{m}}$ for each $n \in \omega$, then $X \subset \bigcup_{n \in \omega} K_{n}$. Moreover, $K_{n}$ is compact in $\beta G$ and contained in $G$ for every $n \in \omega$, which gives us the desired implication.

Now, let $\left\langle\mathscr{U}_{n}: n \in \omega\right\rangle$ be a sequence of open covers of $X$. For each $n \in \omega$ and $U \in \mathscr{U}_{n}$ let $U^{\prime}$ be the open set in $\beta X$ such that $U=U^{\prime} \cap X$. Then we let $A_{n}=\bigcup_{U \in \mathscr{U}_{n}} U^{\prime}$, so that $G=\bigcap_{n \in \omega} A_{n}$ is a Čech-complete subspace of $\beta X$ that contains $X$. Let $\left\{K_{n}: n \in \omega\right\}$ be the collection of compact sets in $\beta X$ contained in $G$ such that $X \subset \bigcup_{n \in \omega} K_{n}$ (we may assume that $K_{n} \subset K_{n+1}$ ). Since $K_{n} \subset G$ for each $n \in \omega$, we may let $\mathscr{F}_{n} \subset \mathscr{U}_{n}$ be the finite subset such that $K_{n} \subset \bigcup_{U \in \mathscr{F}_{n}} U^{\prime}$. Then, clearly, $\left\langle\mathscr{F}_{n}: n \in \omega\right\rangle$ witnesses the Hurewicz property for the sequence $\left\langle\mathscr{U}_{n}: n \in \omega\right\rangle$, which concludes the proof.

Exercise 3.3.28. Write the details of Propositions 3.3.19, 3.3.20 and 3.3.21's proofs. 


\subsubsection{Alster spaces}

We now present yet another covering selection principle that relates to some of the previously mentioned ones and, as we will see in Section 5.3, relates to the productively Lindelöf property. First, let us introduce some concepts:

Definition 3.3.29. Given a space $X$, we say a collection $\mathscr{C} \subset \mathscr{\mathcal { O }}(X)$ is a $K$-cover if for every compact $K \subset X$ there is an $A \in \mathscr{C}$ such that $K \subset A$.

Furthermore, we say $\mathscr{C}$ is an Alster cover if it is a $K$-cover of $G_{\delta}$ subsets of $X$. We denote the family of all Alster covers of a given space by $\mathscr{K}_{\boldsymbol{\delta}}$.

Finally, we say a space $X$ is an Alster space if every Alster cover has a countable subcover.

One can immediately see that the definition of Alster spaces can be formulated in the form of $\left(\begin{array}{c}\mathscr{K}_{\delta} \\ \mathscr{L}_{\delta}\end{array}\right)$, with $\mathscr{L}_{\delta}$ denoting the family of countable $G_{\delta}$ covers. What is surprising, though, is that it can be formulated in the form $S_{1}\left(\mathscr{K}_{\delta}, \mathscr{O}_{\delta}\right)$, with $\mathscr{O}_{\boldsymbol{\delta}}$ denoting the family of all $G_{\delta}$ covers:

Proposition 3.3.30. A space $X$ is Alster if, and only if, $\mathrm{S}_{1}\left(\mathscr{K}_{\delta}, \mathscr{O}_{\delta}\right)$ holds.

Proof. Suppose $X$ is an Alster space and let $\left\langle\mathscr{U}_{n}: n \in \omega\right\rangle$ be a sequence of Alster covers. Let

$$
\mathscr{U}=\bigwedge_{n \in \omega} \mathscr{U}_{n}
$$

Note that $\mathscr{U}$ is an Alster cover, so let $\mathscr{V}=\left\{V_{n}: n \in \omega\right\} \subset \mathscr{U}$ be its countable subcover. Since, for each $n \in \omega, V_{n}=\bigcap_{k \in \omega} U_{n}^{k}$ with $U_{n}^{k} \in \mathscr{U}_{k}$ for all $k \in \omega$, the sequence $\left\langle U_{n}^{n}: n \in \omega\right\rangle$ witnesses $\mathrm{S}_{1}\left(\mathscr{K}_{\delta}, \mathscr{O}_{\delta}\right)$ for the sequence $\left\langle\mathscr{U}_{n}: n \in \omega\right\rangle$, as we wanted to prove.

The other implication is clear.

This characterization is especially useful, because with it we can obtain:

Corollary 3.3.31. If $X$ is an Alster space, then $\mathrm{S}_{\mathrm{fin}}(\mathscr{O}, \mathscr{O})$ holds on $X$.

Proof. Let $\left\langle\mathscr{U}_{n}: n \in \omega\right\rangle$ be a sequence of open covers of $X$. Then, for each $n \in \omega$, let

$$
\mathscr{V}_{n}=\left\{\bigcup \mathscr{F}: \mathscr{F} \subset\left[\mathscr{U}_{n}\right]^{<\omega}\right\}
$$

Note that each $\mathscr{V}_{n}$ is an open $K$-cover, so, by Proposition 3.3.30, we may apply $\mathrm{S}_{1}\left(\mathscr{K}_{\delta}, \mathscr{O}_{\delta}\right)$ to the sequence $\left\langle\mathscr{V}_{n}: n \in \omega\right\rangle$ in order to find a sequence $\left\langle V_{n}: n \in \omega\right\rangle$ of open sets of $X$ such that $V_{n} \in \mathscr{V}_{n}$ and $\bigcup_{n \in \omega} V_{n}=X$. Note that, for every $n \in \omega, V_{n}=\bigcup \mathscr{F}_{n}$ for some finite $\mathscr{F}_{n} \subset \mathscr{U}_{n}$, so $\left\langle\mathscr{F}_{n}: n \in \omega\right\rangle$ witnesses $S_{\text {fin }}(\mathscr{O}, \mathscr{O})$ for $\left\langle\mathscr{U}_{n}: n \in \omega\right\rangle$, and the proof is complete. 
Moreover, using the compactification characterization of Hurewicz spaces presented in Theorem 3.3.27, we can further show that Alster spaces are also Hurewicz spaces. But in order to this, let us first take a step back and introduce a general concept.

Definition 3.3.32. A space $X$ is said to be of countable type if every compact set is included in some compact set of countable character.

Proposition 3.3.33. If $X$ is a Čech-complete space, then $X$ is of countable type.

Proof. Let $X$ be a Čech-complete space, $\left\{A_{n}: n \in \omega\right\}$ be a set of open sets of $\beta X$ such that $X=\bigcap_{n \in \omega} A_{n}$ and fix $K \subset X$ compact. Since $\beta X$ is normal we may find a set of open sets $\left\{B_{n}: n \in \omega\right\}$ of $\beta X$ such that $K \subset \overline{B_{n+1}} \subset B_{n} \subset A_{n}$ for every $n \in \omega$ (with the closure taken in $\beta X)$. Then $K^{\prime}=\bigcap_{n \in \omega} \overline{B_{n}}=\bigcap_{n \in \omega} B_{n}$ is a compact subset of $X$ that contains $K$.

Now, let $V \supset K^{\prime}$ be an open set in $X$. We claim that there is a $k \in \omega$ such that $X \cap B_{k} \subset V$. Indeed, let $V^{\prime}$ be the open set of $\beta X$ such that $V=X \cap V^{\prime}$. Note that $\beta X \backslash V^{\prime}$ is compact and $\left\{\beta X \backslash \overline{B_{n}}: n \in \omega\right\}$ is an increasing open cover for it, so the existence of wished $k \in \omega$ follows, which concludes the proof.

Now, with the right tools at hand, we obtain:

Theorem 3.3.34. If $X$ is a regular Alster space, then $X$ is a Hurewicz space.

Proof. Let $X$ be a regular Alster space. Then, in view of Corollary 3.3.31 and Proposition 3.3.2, $X$ is Lindelöf. Recall that every regular Lindelöf space is normal (see Theorem 1.2.3), so, in order to show that $X$ is Hurewicz, by Theorem 3.3.27, it suffices to show that for every Čech-complete space $Y \supset X$ there is a $\sigma$-compact $F$ such that $X \subset F \subset Y$. Then let $Y$ be a Čech-complete space containing $X$.

Note that, by Proposition 3.3.33, every compact $K \subset X$ is contained in a compact $G_{\delta} K_{\delta} \subset$ $Y$ which, when intersected with $X$, forms a $G_{\delta}$ subset o $X$. Then $\left\{K_{\delta} \cap X: K \subset X\right.$ compact $\}$ is an Alster cover for $X$ and, by picking a countable subcover $\left\{K_{\delta}^{n} \cap X: n \in \omega\right\}$, we conclude that $X$ is contained in a $\sigma$-compact subset of $Y$, as desired.

\subsubsection{The Scheepers Diagram}

One of the most important names in the study of selection principles is that of Marion Scheepers. In his collection of papers "Combinatorics of open covers" he presented a vast amount of results (some of them obtained by other big names like Gerlitz and Nagy) that would later be combined in the renowned Scheepers Diagram. In order to show these results, therefore, we start with the definition of a new family of open covers: 
Definition 3.3.35. Given a space $X$, let $\Omega$ denote the subcollection of $\mathscr{O}$ such that $\mathscr{U} \in \Omega$ if $X \notin \mathscr{U}$ and for every finite $F \subset X$ there is a $U \in \mathscr{U}$ such that $F \subset U$. A cover $\mathscr{U} \in \Omega$ is called an $\omega$-cover.

In view of Proposition 3.1.8, studying the selection principles regarding $\Omega$ and $\Gamma$ would be much easier if $\Gamma \subset \Omega$. This is not the case, however, since the entire space may be a member of a $\gamma$-cover. Then it is in order to bypass this technical nuisance that we define the following auxiliary variation of $\Gamma$, which will be enough to provide us with the desired assistance:

Definition 3.3.36. Given a space $X$, Let $\Gamma^{\prime}=\{\mathscr{U} \backslash\{X\}: \mathscr{U} \in \Gamma\}$.

Proposition 3.3.37. On every space $X$ :

- $\Gamma^{\prime} \subset \Gamma$;

- $\left(\begin{array}{c}\Gamma \\ \Gamma^{\prime}\end{array}\right)$;

- $\Gamma^{\prime} \subset \Omega \subset \mathscr{O}$.

Proof. Proof will be left as an exercise (see 3.3.50).

Now, let us explore some equivalences:

Theorem 3.3.38 ([Scheepers 1996]). Property $\mathrm{S}_{1}(\mathscr{O}, \mathscr{O})$ holds on a space $X$ if, and only if, $\mathrm{S}_{1}(\Omega, \mathscr{O})$ holds on $X$.

Proof. Let $\left\langle\mathscr{U}_{n}: n \in \omega\right\rangle$ be a sequence of open covers of $X$. Fix a partition $\left\{Y_{k}: k \in \omega\right\}$ of $\omega$ with each $Y_{k}$ being infinite. Then, for each $k \in \omega$, set

$$
\mathscr{V}_{k}=\left\{\bigcup_{i \in Y_{k}} U_{i}: U_{i} \in \mathscr{U}_{i}\right\} .
$$

Note that if $X \in \mathscr{V}_{k}$ for some $k \in \omega$, then the proof is complete, so we may assume without loss of generality that, for every $k \in \omega, \mathscr{V}_{k} \in \Omega$. In this case, there must be a $V_{k} \in \mathscr{V}_{k}$ for each $k \in \omega$ such that $\bigcup_{k \in \omega} V_{k}=X$. Note that, for each $i \in Y_{k}$ there is a $U_{i} \in \mathscr{U}_{i}$ such that $V_{k}=\bigcup_{i \in Y_{k}} U_{i}$. Then, clearly, $\left\langle U_{n}: n \in \omega\right\rangle$ witnesses $S_{1}(\mathscr{O}, \mathscr{O})$ for $\left\langle\mathscr{U}_{n}: n \in \omega\right\rangle$.

The other implication follows from Proposition 3.1.8.

Theorem 3.3.39. The following properties are equivalent on Lindelöf spaces:

- $\mathrm{S}_{\mathrm{fin}}(\mathscr{O}, \mathscr{O})$;

- $\mathrm{S}_{\text {fin }}(\Omega, \mathscr{O})$;

- $\mathrm{S}_{\mathrm{fin}}(\Gamma, \mathscr{O})$. 
Proof. In view of Propositions 3.1.8 and 3.3.37, it is clear that

$$
\mathrm{S}_{\text {fin }}(\mathscr{O}, \mathscr{O}) \Longrightarrow \mathrm{S}_{\text {fin }}(\Omega, \mathscr{O}) \Longrightarrow \mathrm{S}_{\text {fin }}(\Gamma, \mathscr{O})
$$

so let $X$ be a Lindelöf space such that $S_{\text {fin }}(\Gamma, \mathscr{O})$ holds on $X$ and let $\left\langle\mathscr{U}_{n}: n \in \omega\right\rangle$ be a sequence of open covers of $X$ (since $X$ is Lindelöf, we may assume that $\mathscr{U}_{n}=\left\{U_{n}^{k}: k \in \omega\right\}$ ). For each $n \in \omega$, let

$$
\mathscr{V}_{n}=\left\{\bigcup_{i \leq k} U_{n}^{i}: k \in \omega\right\} .
$$

Note that, since each $\mathscr{V}_{n}$ is an increasing cover, $\mathscr{V}_{n}$ is finite if, and only if, $X \in \mathscr{V}_{n}$, which would conclude the proof. In this case, without loss of generality, we may assume that $\left\langle\mathscr{V}_{n}: n \in \omega\right\rangle$ is a sequence of $\gamma$-covers, so that by applying $\mathrm{S}_{\text {fin }}(\Gamma, \mathscr{O})$ to $\left\langle\mathscr{V}_{n}: n \in \omega\right\rangle$ we get a sequence $\left\langle k_{n}: n \in \omega\right\rangle$ such that, by letting $\mathscr{F}_{n}=\left\{U_{n}^{i}: i \leq k_{n}\right\} \subset \mathscr{U}_{n}, \bigcup_{n \in \omega} \mathscr{F}_{n} \in \mathscr{O}$, and the proof is complete.

Theorem 3.3.40 ([Gerlits and Nagy 1982]). Let X be a $T_{1}$ space. Then the following properties are equivalent on $X$ :

- $\mathrm{S}_{1}(\Omega, \Gamma)$;

- $\mathrm{S}_{\text {fin }}(\Omega, \Gamma)$;

- $\left(\begin{array}{l}\Omega \\ \Gamma\end{array}\right)$.

Proof. By Proposition 3.1.9, even when $X$ is not $T_{1}$,

$$
\mathrm{S}_{1}(\Omega, \Gamma) \Longrightarrow \mathrm{S}_{\text {fin }}(\Omega, \Gamma) \Longrightarrow\left(\begin{array}{c}
\Omega \\
\Gamma
\end{array}\right)
$$

so it suffices to show that $\left(\begin{array}{l}\Omega \\ \Gamma\end{array}\right) \Longrightarrow \mathrm{S}_{1}(\Omega, \Gamma)$.

Note that if $X$ is finite, then $\Omega=\emptyset$ and, therefore, the result follows trivially. So suppose $X$ is infinite, $\left(\begin{array}{c}\Omega \\ \Gamma\end{array}\right)$ holds and let $\left\langle\mathscr{U}_{n}: n \in \omega\right\rangle$ be a sequence of covers from $\Omega$ (we may assume $\mathscr{U}_{n+1}$ is a refinement of $\left.\mathscr{U}_{n}\right)$. Fix $\left\{x_{n}: n \in \omega\right\} \subset X$ such that $x_{i} \neq x_{j}$ whenever $i \neq j$. Then

$$
\mathscr{U}=\bigcup_{n \in \omega}\left\{U \backslash\left\{x_{n}\right\}: U \in \mathscr{U}_{n}\right\}
$$

is an $\omega$-cover (because $X$ is $T_{1}$ ), so let $\mathscr{V}=\left\{V_{k}: k \in \omega\right\}$ be its $\gamma$-subcover. For each $k \in \omega$, let $n_{k} \in \omega$ be such that $V_{k}=U_{n_{k}} \backslash\left\{x_{n_{k}}\right\}$ for some $U_{n_{k}} \in \mathscr{U}_{n_{k}}$. Without loss of generality, we may assume (taking a subcover from $\mathscr{V}$, if necessary) that $n_{i} \neq n_{j}$ whenever $i \neq j$. For $n \leq n_{0}$, pick $U_{n} \in \mathscr{U}_{n}$ such that $V_{0} \subset U_{n} \backslash\left\{x_{n_{0}}\right\}$ (this is possible because $\mathscr{U}_{n+1}$ is a refinement of $\mathscr{U}_{n}$ ). Now, given $k \in \omega$, for every $n_{k}<n \leq n_{k+1}$, pick $U_{n} \in \mathscr{U}_{n}$ such that $V_{k+1} \subset U_{n} \backslash\left\{x_{n_{k+1}}\right\}$. Clearly, $\left\langle U_{n}: n \in \omega\right\rangle$ witnesses $S_{1}(\Omega, \Gamma)$ for $\left\langle\mathscr{U}_{n}: n \in \omega\right\rangle$.

In some cases these selection principles may be trivial: 
Proposition 3.3.41. If $X$ is a $T_{1}$ space such that $|X| \geq 2$, then $\left(\begin{array}{l}\mathscr{O} \\ \Omega\end{array}\right)$ does not hold.

Proof. Let $x, y \in X$ be two distinct points. Then, clearly, $\{X \backslash\{x\}, X \backslash\{y\}\}$ witnesses that $\left(\begin{array}{l}\mathscr{O} \\ \Omega\end{array}\right)$ does not hold.

Corollary 3.3.42. If $X$ is a $T_{1}$ space, then $\left(\begin{array}{c}\mathscr{O} \\ \Gamma\end{array}\right)$ does not hold.

Proof. If $X$ is finite, then $\Gamma=\emptyset$, so $\left(\begin{array}{c}\mathscr{O} \\ \Gamma\end{array}\right)$ does not hold. On the other hand, if $X$ is infinite, then $\left(\begin{array}{l}\mathscr{O} \\ \Gamma\end{array}\right)$ does not hold by Propositions 3.1.8, 3.3.37 and 3.3.41.

And we also have some interesting results involving $U_{\text {fin }}$ :

Proposition 3.3.43. If $X$ is a Lindelöf space, then, for every $\mathscr{B} \in\{\mathscr{O}, \Omega, \Gamma\}$,

$$
\mathrm{S}_{1}(\Gamma, \mathscr{B}) \Longrightarrow \mathrm{S}_{\text {fin }}(\Gamma, \mathscr{B}) \Longrightarrow \mathrm{U}_{\text {fin }}(\mathscr{O}, \mathscr{B})
$$

Proof. It is clear that $\mathrm{S}_{1}(\Gamma, \mathscr{B}) \Longrightarrow \mathrm{S}_{\text {fin }}(\Gamma, \mathscr{B})$, so suppose $\mathrm{S}_{\text {fin }}(\Gamma, \mathscr{B})$ holds and let $\left\langle\mathscr{U}_{n}: n \in \omega\right\rangle$ be a sequence of open covers. Since $X$ is Lindelöf, we may assume $\mathscr{U}_{n}=\left\{U_{n}^{k}: k \in \omega\right\}$ for each $n \in \omega$. Also, if $\mathscr{U}_{n}$ has a finite subcover for some $n \in \omega$, then it is clear that $\mathrm{U}_{\text {fin }}(\mathscr{O}, \mathscr{B})$ holds for $\left\langle\mathscr{U}_{n}: n \in \omega\right\rangle$, so assume $\mathscr{U}_{n}$ has no finite subcover for all $n \in \omega$ and set

$$
\mathscr{V}_{n}=\left\{\bigcup_{k \leq m} U_{n}^{k}: m \in \omega\right\} .
$$

Note that, with all our assumptions, $\mathscr{V}_{n} \in \Gamma$ for all $n \in \omega$ (because they are increasing open covers), so we find finite sets $\mathscr{F}_{n}^{\prime} \subset \mathscr{V}_{n}$ such that $\bigcup_{n \in \omega} \mathscr{F}_{n}^{\prime} \in \mathscr{B}$. Since $\mathscr{V}_{n}$ is an increasing open cover, there is, in fact, a $U_{n} \in \mathscr{F}_{n}^{\prime}$ such that $U \subset U_{n}$ for all $U \in \mathscr{F}_{n}^{\prime}$, so let $m_{n}$ be such that $U_{n}=\bigcup_{k \leq m_{n}} U_{n}^{k}$ and set $\mathscr{F}_{n}=\left\{U_{n}^{k}: k \leq m_{n}\right\}$. Clearly, $\left\langle\mathscr{F}_{n}: n \in \omega\right\rangle$ attests $\bigcup_{\text {fin }}(\mathscr{O}, \mathscr{B})$ for $\left\langle\mathscr{U}_{n}: n \in \omega\right\rangle$.

Proposition 3.3.44. For every $\mathscr{B} \in\{\mathscr{O}, \Omega, \Gamma\}$, then

$$
\mathrm{U}_{\text {fin }}(\mathscr{O}, \mathscr{B}) \Longleftrightarrow \mathrm{U}_{\text {fin }}(\Omega, \mathscr{B}) \Longrightarrow \mathrm{U}_{\text {fin }}(\Gamma, \mathscr{B})
$$

Moreover, if $X$ is an infinite Lindelöf $T_{1}$ space, then all of the above properties are equivalent.

Proof. By Proposition 3.1.8,

$$
\mathrm{U}_{\text {fin }}(\mathscr{O}, \mathscr{B}) \Longrightarrow \mathrm{U}_{\text {fin }}(\Omega, \mathscr{B}) \Longrightarrow \mathrm{U}_{\text {fin }}(\Gamma, \mathscr{B})
$$

so suppose $\mathrm{U}_{\text {fin }}(\Omega, \mathscr{B})$ holds and let $\left\langle\mathscr{U}_{n}: n \in \omega\right\rangle$ be a sequence of open covers. If $\mathscr{U}_{n}$ has a finite subcover for some $n \in \omega$, then it is clear that $\mathrm{U}_{\text {fin }}(\mathscr{O}, \mathscr{B})$ holds for $\left\langle\mathscr{U}_{n}: n \in \omega\right\rangle$, so assume $\mathscr{U}_{n}$ has no finite subcover for all $n \in \omega$ and set

$$
\mathscr{V}_{n}=\left\{\bigcup \mathscr{F}: \mathscr{F} \subset \mathscr{U}_{n} \text { finite }\right\} .
$$


Then $\mathscr{V}_{n} \in \Omega$ for every $n \in \omega$ and, by applying $\mathrm{U}_{\text {fin }}(\Omega, \mathscr{B})$ to the sequence $\left\langle\mathscr{V}_{n}: n \in \omega\right\rangle$, we find a sequence of finite subsets $\left\langle\mathscr{F}_{n}: n \in \omega\right\rangle$ that witnesses $\bigcup_{\text {fin }}(\mathscr{O}, \mathscr{B})$ for $\left\langle\mathscr{U}_{n}: n \in \omega\right\rangle$.

Now, assume that $X$ is Lindelöf and that $\mathrm{U}_{\text {fin }}(\Gamma, \mathscr{B})$ holds. Let $\left\langle\mathscr{U}_{n}: n \in \omega\right\rangle$ be a sequence of open covers such that $\mathscr{U}_{n}=\left\{U_{n}^{k}: k \in \omega\right\}$ for every $n \in \omega$. If $\mathscr{U}_{n}$ has a finite subcover for some $n \in \omega$, then it is clear that $\bigcup_{\text {fin }}(\mathscr{O}, \mathscr{B})$ holds for $\left\langle\mathscr{U}_{n}: n \in \omega\right\rangle$, so assume $\mathscr{U}_{n}$ has no finite subcover for all $n \in \omega$ and set

$$
\mathscr{V}_{n}=\left\{V_{n}^{k}=\bigcup_{i \leq k} U_{n}^{k}: k \in \omega\right\}
$$

Note that $\mathscr{V}_{n} \in \Gamma$ for all $n \in \omega$, so we may apply $\mathrm{U}_{\text {fin }}(\Gamma, \mathscr{B})$ to the sequence $\left\langle\mathscr{V}_{n}: n \in \omega\right\rangle$ in order to obtain, for each $n \in \omega$, a finite set $\mathscr{F}_{n}^{\prime} \subset \mathscr{V}_{n}$ such that $\left\{\bigcup \mathscr{F}_{n}^{\prime}: n \in \omega\right\} \in \Gamma$. In fact, since each $\mathscr{V}_{n}$ is an increasing cover, we may find a $k_{n} \in \omega$ for each $n \in \omega$ such that $V_{n}^{k_{n}}=\bigcup \mathscr{F}_{n}^{\prime}$, so $\left\{V_{n}^{k_{n}}: n \in \omega\right\} \in \Gamma$. If we let $\mathscr{F}_{n}=\left\{U_{n}^{j}: j \leq k_{n}\right\}$, then it is clear that $\left\langle\mathscr{F}_{n}: n \in \omega\right\rangle$ witnesses $\mathrm{U}_{\text {fin }}(\mathscr{O}, \mathscr{B})$ for $\left\langle\mathscr{U}_{n}: n \in \omega\right\rangle$.

We finish our framework of results leaning towards the Scheepers Diagram with the following:

Theorem 3.3.45 ([Just et al. 1996]). For every space $X, \mathrm{~S}_{1}(\Gamma, \Gamma)$ holds if, and only if, $\mathrm{S}_{\mathrm{fin}}(\Gamma, \Gamma)$ holds.

Proof. By Proposition 3.1.9, $\mathrm{S}_{1}(\Gamma, \Gamma) \Longrightarrow \mathrm{S}_{\mathrm{fin}}(\Gamma, \Gamma)$, so suppose $\mathrm{S}_{\mathrm{fin}}(\Gamma, \Gamma)$ holds and let $\left\langle\mathscr{U}_{n}: n \in \omega\right\rangle$ be a sequence of $\gamma$-covers. For each $n \in \omega$, fix an enumeration $\mathscr{U}_{n}=\left\{U_{n}^{k}: k \in \omega\right\}$ and then set

$$
\mathscr{V}_{n}=\left\{V_{n}^{k}=\bigcap_{i \leq n} U_{i}^{k}: k \in \omega\right\} .
$$

Note that $\mathscr{V}_{n}$ is a $\gamma$-cover for every $n \in \omega$, so apply $S_{\text {fin }}(\Gamma, \Gamma)$ to the sequence $\left\langle\mathscr{V}_{n}: n \in \omega\right\rangle$ to find finite sets $\mathscr{W}_{n} \subset \mathscr{V}_{n}$ such that $\mathscr{W}=\bigcup_{n \in \omega} \mathscr{W}_{n} \in \Gamma$. Note that we may assume that $\mathscr{W}_{i} \cap \mathscr{W}_{j}=\emptyset$ for all $i, j \in \omega$ such that $i \neq j$ and then, since each $\mathscr{W}_{n}$ is finite and $\mathscr{W}$ is infinite, we may find a strictly increasing sequence of natural numbers $\left\langle n_{j}: j \in \omega\right\rangle$ such that $\mathscr{W}_{n_{j}}$ is nonempty. Then, for each $j \in \omega$, fix $m_{j}$ such that $V_{n_{j}}^{m_{j}} \in \mathscr{W}_{n_{j}}$. In this case, $\left\{V_{n_{j}}^{m_{j}}: j \in \omega\right\}$ is an infinite subset of $\mathscr{W}$, so it follows from Corollary 3.3.23 that $\left\{V_{n_{j}}^{m_{j}}: j \in \omega\right\} \in \Gamma$. For each $n \leq n_{0}$, let $U_{n}=U_{n}^{m_{0}}$. Then, for each $k \in \omega$ and $n \in \omega$ such that $n_{k}<n \leq n_{k+1}$, let $U_{n}=U_{n}^{m_{k+1}}$.

We claim that $\left\{U_{n}: n \in \omega\right\} \in \Gamma$. Indeed, note that $U_{n_{k}}=V_{n_{k}}^{m_{k}}$, so $\left\{U_{n}: n \in \omega\right\}$ must be infinite (since $\left\{V_{n_{k}}^{m_{k}}: k \in \omega\right\}$ is infinite). On the other hand, fix $x \in X$. Then there is an $N_{x} \in \omega$ such that $x \in V_{n_{k}}^{m_{k}}$ for all $k \geq N_{x}$. In this case, since $U_{n} \supset V_{n_{k+1}}^{m_{k+1}}$ for all $n_{k}<n \leq n_{k+1}$, it follows that $x \in U_{n}$ for all $n \geq n_{N_{x}+1}$. So $\left\{U_{n}: n \in \omega\right\} \in \Gamma$.

Theorem 3.3.46 (Scheepers Diagram). The following diagram (with each arrow representing an implication) is true for every Lindelöf $T_{1}$ space $X$. 


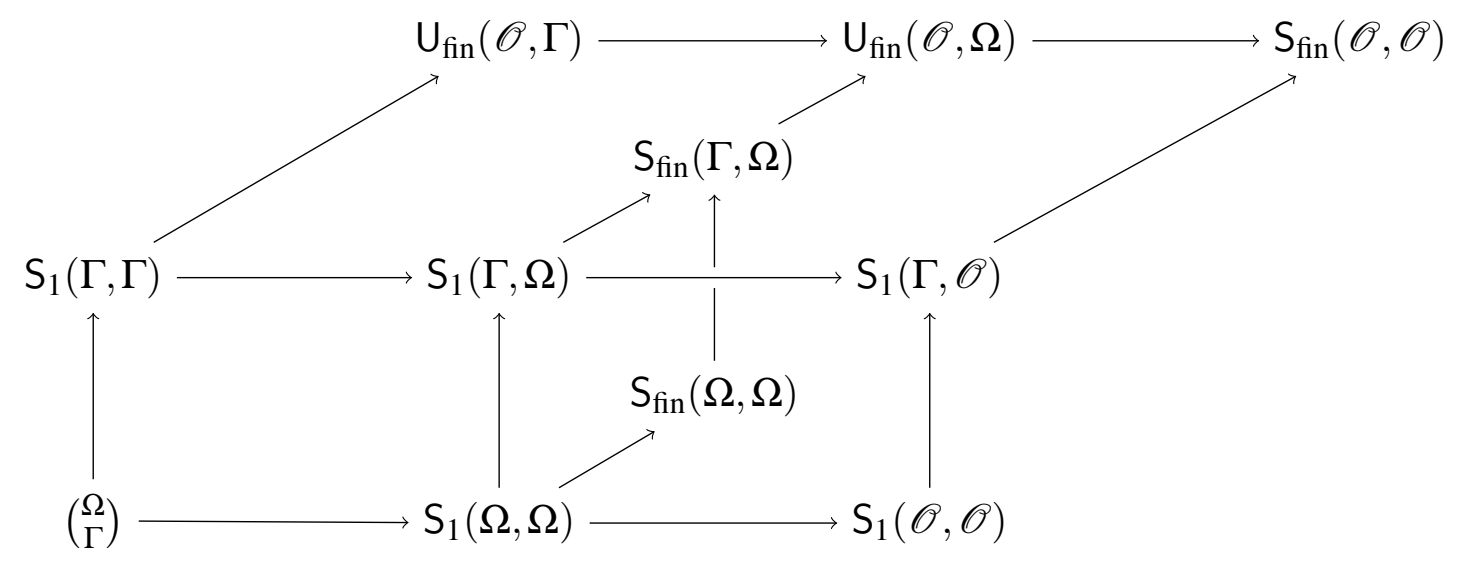

Moreover, if $|X| \geq 2, \mathscr{A}, \mathscr{B} \in\{\mathscr{O}, \Omega, \Gamma\}$ and $\mathrm{P} \in\left\{(), \mathrm{S}_{1}, \mathrm{~S}_{\mathrm{fin}}, \mathrm{U}_{\mathrm{fin}}\right\}$, then one of the following holds:

- $\mathrm{P}(\mathscr{A}, \mathscr{B})$ never holds;

- $\mathrm{P}(\mathscr{A}, \mathscr{B})$ always holds;

- $\mathrm{P}(\mathscr{A}, \mathscr{B})$ is equivalent to one of the selection principles in the diagram.

Proof. Each implication in the diagram follows from a combination of Propositions 3.1.8, 3.1.9, 3.3.19, 3.3.20, 3.3.21, 3.3.37, 3.3.43 and Theorems 3.3.38, 3.3.40.

Now, suppose $|X| \geq 2$. Then, by Propositions 3.1.9, 3.3.41 and Corollary 3.3.42, $\mathrm{P}(\mathscr{O}, \mathscr{B})$ never holds for all $\mathscr{B} \in\{\Omega, \Gamma\}$ and $\mathrm{P} \in\left\{(), \mathrm{S}_{1}, \mathrm{~S}_{\text {fin }}\right\}$.

On the other hand, $\left(\begin{array}{c}\mathscr{A} \\ \mathscr{B}\end{array}\right)$ always holds for all $\mathscr{A} \in\{\mathscr{O}, \Omega, \Gamma\}$ and $\mathscr{B} \in\{\mathscr{O}, \Omega, \Gamma\}$ such that $\mathscr{B} \subset \mathscr{A}$.

Then it remains to show that each of the following properties are equivalent to some of the selection principles in the diagram: $\mathrm{S}_{1}(\Omega, \Gamma), \mathrm{S}_{\mathrm{fin}}(\Omega, \Gamma), \mathrm{S}_{1}(\Omega, \mathscr{O}), \mathrm{S}_{\mathrm{fin}}(\Omega, \mathscr{O}), \mathrm{S}_{\mathrm{fin}}(\Gamma, \mathscr{O})$, $\mathrm{S}_{\text {fin }}(\Gamma, \Gamma), \mathrm{U}_{\text {fin }}(\Gamma, \Omega), \mathrm{U}_{\text {fin }}(\Omega, \Omega), \mathrm{U}_{\text {fin }}(\Gamma, \Gamma), \mathrm{U}_{\text {fin }}(\Omega, \Gamma), \mathrm{U}_{\text {fin }}(\Gamma, \mathscr{O}), \mathrm{U}_{\text {fin }}(\Omega, \mathscr{O}), \mathrm{U}_{\text {fin }}(\mathscr{O}, \mathscr{O})$.

- By Theorem 3.3.40, $\mathrm{S}_{1}(\Omega, \Gamma)$ and $\mathrm{S}_{\text {fin }}(\Omega, \Gamma)$ are equivalent to $\left(\begin{array}{c}\Omega \\ \Gamma\end{array}\right)$;

- By Theorem 3.3.38, $S_{1}(\Omega, \mathscr{O})$ is equivalent to $S_{1}(\mathscr{O}, \mathscr{O})$;

- By Theorem 3.3.39, $\mathrm{S}_{\text {fin }}(\Omega, \mathscr{O})$ and $\mathrm{S}_{\text {fin }}(\Gamma, \mathscr{O})$ are equivalent to $\mathrm{S}_{\text {fin }}(\mathscr{O}, \mathscr{O})$;

- By Theorem 3.3.45, $S_{\text {fin }}(\Gamma, \Gamma)$ is equivalent to $S_{1}(\Gamma, \Gamma)$;

- By Proposition 3.3.44, $\mathrm{U}_{\text {fin }}(\Gamma, \Omega)$ and $\mathrm{U}_{\text {fin }}(\Omega, \Omega)$ are both equivalent to $\mathrm{U}_{\text {fin }}(\mathscr{O}, \Omega)$;

- By Proposition 3.3.44, $\mathrm{U}_{\text {fin }}(\Gamma, \Gamma)$ and $\mathrm{U}_{\text {fin }}(\Omega, \Gamma)$ are both equivalent to $\mathrm{U}_{\text {fin }}(\mathscr{O}, \Gamma)$;

- By Proposition 3.3.44, $\mathrm{U}_{\text {fin }}(\Gamma, \mathscr{O})$ and $\mathrm{U}_{\text {fin }}(\Omega, \mathscr{O})$ are both equivalent to $\mathrm{U}_{\text {fin }}(\mathscr{O}, \mathscr{O})$ which, by Proposition 3.3.21, is equivalent to $\mathrm{S}_{\text {fin }}(\mathscr{O}, \mathscr{O})$. 
Before finishing this section we will show yet another characterization involving Rothberger spaces. In order to do that, consider the following simple lemma.

Lemma 3.3.47. Given a space $X$, consider $k \in \mathbb{N}$, a finite $F \subset X$ and an open set $U \subset X^{k}$ (in the product topology). If $F^{k} \subset U$, then there is an open set $V \subset X$ such that $F^{k} \subset V^{k} \subset U$ (in particular, $F \subset V$ ).

Proof. Let $F=\left\{x_{i}: i \leq n\right\}$. Note that for each $v \in F^{k}$ there is a box $B_{v}=\prod_{j \leq k} U_{j}$ such that $v \in B_{v} \subset U$. Given $i \leq k$, let $\pi_{i}: X^{k} \rightarrow X$ denote the projection in the $i$ th coordinate and then, for each $x \in F$, let

$$
V_{x}=\bigcap\left\{\pi_{i}\left[B_{v}\right]: v \in F^{k} \text { and } i \leq k \text { with } x=\pi_{i}(v)\right\},
$$

so that $V_{x} \subset X$ is an open neighborhood of $x$. We leave as an exercise to show that $V=\bigcup_{x \in F} V_{x}$ has the desired property.

Theorem 3.3.48 ([Sakai 1988]). Property $\mathrm{S}_{1}(\Omega, \Omega)$ holds on a space $X$ if, and only if, $\mathrm{S}_{1}(\mathscr{O}, \mathscr{O})$ holds on $X^{k}$ for every $k \in \mathbb{N}$.

Proof. Suppose $\mathrm{S}_{1}(\Omega, \Omega)$ holds on $X$. Then, by Theorem 3.3.46, $\mathrm{S}_{1}(\mathscr{O}, \mathscr{O})$ holds on $X$, so in order to show the first implication it suffices to show that $\mathrm{S}_{1}(\Omega, \Omega)$ holds on $X^{k}$ for every $k \in \mathbb{N}$. Let $k \in \mathbb{N}$ and $\left\langle\mathscr{U}_{n}: n \in \omega\right\rangle$ be a sequence of $\omega$-covers in $X^{k}$. For each $n \in \omega$, let

$$
\mathscr{V}_{n}=\left\{V \subset X: V^{k} \subset U \text { for some } U \in \mathscr{U}_{n} \text { and } V \text { is open }\right\} .
$$

Since $\mathscr{U}_{n} \in \Omega$, it follows from Lemma 3.3.47 that $\mathscr{V}_{n} \in \Omega$ for every $n \in \omega$. In this case, let $\left\langle V_{n}: n \in \omega\right\rangle$ be the sequence witnessing $S_{1}(\Omega, \Omega)$ for $\left\langle\mathscr{V}_{n}: n \in \omega\right\rangle$ and fix, for each $n \in \omega$, $U_{n} \in \mathscr{U}_{n}$ such that $V_{n}^{k} \subset U_{n}$. Then $\left\langle U_{n}: n \in \omega\right\rangle$ witnesses $\mathrm{S}_{1}(\Omega, \Omega)$ for $\left\langle\mathscr{U}_{n}: n \in \omega\right\rangle$, as desired.

On the other hand, suppose $\mathrm{S}_{1}(\mathscr{O}, \mathscr{O})$ holds on $X^{k}$ for every $k \in \mathbb{N}$. Then, by Proposition 3.3.14, $\mathrm{S}_{1}(\mathscr{O}, \mathscr{O})$ holds over the disjoint union $Y=\bigcup_{k \in \mathbb{N}} X^{k}$. Let $\left\langle\mathscr{U}_{n}: n \in \omega\right\rangle$ be a sequence of $\omega$-covers in $X$. For each $n \in \omega$, let

$$
\mathscr{V}_{n}=\bigcup_{k \in \omega}\left\{V^{k}: V \in \mathscr{U}_{n}\right\}
$$

Since $\mathscr{U}_{n} \in \Omega, \mathscr{V}_{n}$ is an open cover of $Y$, so let $\left\langle V_{n}^{k_{n}}: n \in \omega\right\rangle$ be the sequence witnessing $\mathrm{S}_{1}(\mathscr{O}, \mathscr{O})$ over $Y$ for $\left\langle\mathscr{V}_{n}: n \in \omega\right\rangle$. Then, clearly, $\left\langle V_{n}: n \in \omega\right\rangle$ witnesses $S_{1}(\Omega, \Omega)$ over $X$ for $\left\langle\mathscr{U}_{n}: n \in \omega\right\rangle$, and the proof is complete.

The following Theorem can be shown following the steps of Theorem 3.3.48, so its proof will be left as an exercise (3.3.52).

Theorem 3.3.49. Property $\mathrm{S}_{\mathrm{fin}}(\Omega, \Omega)$ holds on a space $X$ if, and only if, $\mathrm{S}_{\mathrm{fin}}(\mathscr{O}, \mathscr{O})$ holds on $X^{k}$ for every $k \in \mathbb{N}$. 
Exercise 3.3.50. Write the details of Theorem 3.3.37's proof.

Exercise 3.3.51. Pinpoint what combination of results from Propositions 3.1.8, 3.1.9, 3.3.19, 3.3.20, 3.3.21, 3.3.43 and Theorems 3.3.38, 3.3.40 show each implication from the Scheepers Diagram 3.3.46.

Exercise 3.3.52. Write the details of Theorem 3.3.49's proof. 
CHAPTER

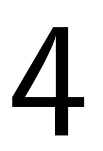

THE ASSOCIATED SELECTIVE GAMES

Now we finally turn our attention back to games! In this chapter we will see that most of the selection principles presented in the previous chapter give rise to a new game, so we will focus on studying these new games.

They go as follows:

Definition 4.0.1. Given nonempty families $\mathscr{A}, \mathscr{B}$, with $\emptyset \notin \mathscr{A}$, we denote by $\mathrm{G}_{1}(\mathscr{A}, \mathscr{B})$ the following game. On each inning $n \in \omega$,

- Alice chooses $A_{n} \in \mathscr{A}$;

- and Bов chooses $b_{n} \in A_{n}$.

We say that Вов wins if $\left\{b_{n}: n \in \omega\right\} \in \mathscr{B}$ and AlICE wins otherwise.

As a first example we present the Rothberger game:

Example 4.0.2. The game $G_{1}(\mathscr{O}, \mathscr{O})$ is called the Rothberger game. In other words, $G_{1}(\mathscr{O}, \mathscr{O})$ is the game in which, in each inning $n \in \omega$,

- ALICE chooses an open cover $\mathscr{U}_{n}$ of $X$;

- and Bов chooses an open set $U_{n} \in \mathscr{U}_{n}$,

so that BоB wins if $\left\{U_{n}: n \in \omega\right\}$ is an open cover of $X$ and ALICE wins otherwise.

The game $\mathrm{G}_{1}(\mathscr{A}, \mathscr{B})$ and the selection principle $\mathrm{S}_{1}(\mathscr{A}, \mathscr{B})$ share a relation that goes beyond the similarity in the notation:

Proposition 4.0.3. Given nonempty families $\mathscr{A}, \mathscr{B}$, if ALICE does not have a winning strategy in the game $\mathrm{G}_{1}(\mathscr{A}, \mathscr{B})$, then $\mathrm{S}_{1}(\mathscr{A}, \mathscr{B})$ holds. 
Proof. Suppose $\mathrm{S}_{1}(\mathscr{A}, \mathscr{B})$ does not hold. Then there is a sequence $\left\langle A_{n}: n \in \omega\right\rangle$ of elements of $\mathscr{A}$ such that, for every sequence $\left\langle b_{n}: n \in \omega\right\rangle$ with each $b_{n}$ being an element of $A_{n},\left\{b_{n}: n \in \omega\right\} \notin \mathscr{B}$. Clearly, if ALICE plays with $\left\langle A_{n}: n \in \omega\right\rangle$ (regardless of BoB's responses) in $\mathrm{G}_{1}(\mathscr{A}, \mathscr{B})$, then AlicE wins.

Definition 4.0.4. Given nonempty families $\mathscr{A}, \mathscr{B}$, with $\emptyset \notin \mathscr{A}$, and $k \in \mathbb{N}$ such that $k \geq 2$, we denote by $\mathrm{G}_{\mathrm{k}}(\mathscr{A}, \mathscr{B})$ the following game . On each inning $n \in \omega$, we have:

- AliCE chooses $A_{n} \in \mathscr{A}$;

- BoB chooses $B_{n} \subset A_{n}$ such that $\left|B_{n}\right| \leq k$.

We say that Bов wins if $\bigcup_{n \in \omega} B_{n} \in \mathscr{B}$ and ALICE wins otherwise.

Example 4.0.5. The game $\mathrm{G}_{\mathrm{k}}(\mathscr{O}, \mathscr{O})$ is called the $\boldsymbol{k}$-Rothberger game. In other words, $\mathrm{G}_{\mathrm{k}}(\mathscr{O}, \mathscr{O})$ is the game in which, in each inning $n \in \omega$,

- ALICE chooses an open cover $\mathscr{U}_{n}$ of $X$;

- and BoB chooses an $\mathscr{F}_{n} \subset \mathscr{U}_{n}$ such that $\left|\mathscr{F}_{n}\right| \leq k$,

so that BOB wins if $\bigcup_{n \in \omega} \mathscr{F}_{n}$ is an open cover of $X$ and ALICE wins otherwise.

Analogously to Proposition 4.0.3 (Exercise 4.0.12), we also have:

Proposition 4.0.6. Given nonempty families $\mathscr{A}, \mathscr{B}$ and $k \in \mathbb{N}$, if ALICE does not have a winning strategy in the game $\mathrm{G}_{\mathrm{k}}(\mathscr{A}, \mathscr{B})$, then $\mathrm{S}_{\mathrm{k}}(\mathscr{A}, \mathscr{B})$ holds.

And, finally:

Definition 4.0.7. Given nonempty families $\mathscr{A}, \mathscr{B}$, with $\emptyset \notin \mathscr{A}$, we denote by $\mathrm{G}_{\text {fin }}(\mathscr{A}, \mathscr{B})$ the following game: in each inning $n \in \omega$,

- AliCE chooses $A_{n} \in \mathscr{A}$;

- Bob chooses a finite $B_{n} \subset A_{n}$.

We say that Вов wins if $\bigcup_{n \in \omega} B_{n} \in \mathscr{B}$ and that ALICE wins otherwise.

Example 4.0.8. The game $\mathrm{G}_{\mathrm{fin}}(\mathscr{O}, \mathscr{O})$ is called the Menger game. In other words, $\mathrm{G}_{\mathrm{fin}}(\mathscr{O}, \mathscr{O})$ is the game in which, in each inning $n \in \omega$,

- AliCE chooses an open cover $\mathscr{U}_{n}$ of $X$;

- and Bов chooses a finite $\mathscr{F}_{n} \subset \mathscr{U}_{n}$, 
so that BОВ wins if $\bigcup_{n \in \omega} \mathscr{F}_{n}$ is an open cover of $X$ and ALICE wins otherwise.

Once again, we immediately have (Exercise 4.0.12):

Proposition 4.0.9. Given nonempty families $\mathscr{A}, \mathscr{B}$, if ALICE does not have a winning strategy in the game $\mathrm{G}_{\mathrm{fin}}(\mathscr{A}, \mathscr{B})$, then $\mathrm{S}_{\mathrm{fin}}(\mathscr{A}, \mathscr{B})$ holds.

The following trivial results will also be useful for later proofs.

Proposition 4.0.10. Let $\mathscr{A}$ and $\mathscr{B}$ be nonempty families of sets with $\emptyset \notin \mathscr{A}$. If $\mathscr{C} \subset \mathscr{A}$ and $\mathscr{D} \subset \mathscr{B}$ are nonempty, then:

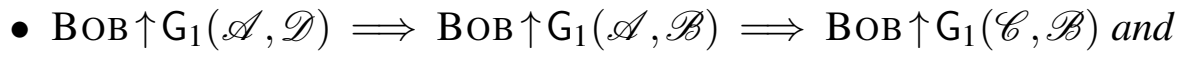

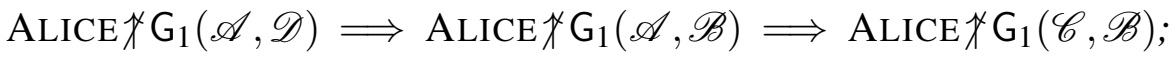

- for every $k \geq 2$ :

$\mathrm{BoB} \uparrow \mathrm{G}_{\mathrm{k}}(\mathscr{A}, \mathscr{D}) \Longrightarrow \mathrm{BoB} \uparrow \mathrm{G}_{\mathrm{k}}(\mathscr{A}, \mathscr{B}) \Longrightarrow \mathrm{BoB} \uparrow \mathrm{G}_{\mathrm{k}}(\mathscr{C}, \mathscr{B})$ and

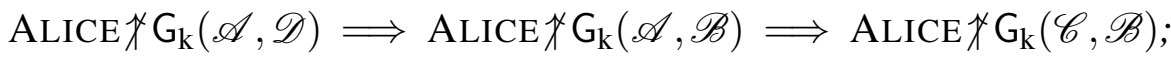

- $\mathrm{BoB} \uparrow \mathrm{G}_{\mathrm{fin}}(\mathscr{A}, \mathscr{D}) \Longrightarrow \mathrm{BoB} \uparrow \mathrm{G}_{\text {fin }}(\mathscr{A}, \mathscr{B}) \Longrightarrow \mathrm{BoB} \uparrow \mathrm{G}_{\text {fin }}(\mathscr{C}, \mathscr{B})$ and $\operatorname{ALICE} \nmid G_{\text {fin }}(\mathscr{A}, \mathscr{D}) \Longrightarrow \operatorname{ALICE} \nmid \mathrm{G}_{\mathrm{fin}}(\mathscr{A}, \mathscr{B}) \Longrightarrow \operatorname{ALICE} \nmid \mathrm{G}_{\mathrm{fin}}(\mathscr{C}, \mathscr{B})$.

Proposition 4.0.11. Let $\mathscr{A}$ and $\mathscr{B}$ be nonempty families of sets with $\emptyset \notin \mathscr{A}$. Then

$$
\begin{aligned}
& \mathrm{BOB} \uparrow \mathrm{G}_{1}(\mathscr{A}, \mathscr{B}) \Longrightarrow \mathrm{BoB} \uparrow \mathrm{G}_{\mathrm{k}}(\mathscr{A}, \mathscr{B}) \Longrightarrow \mathrm{BoB} \uparrow \mathrm{G}_{\text {fin }}(\mathscr{A}, \mathscr{B}) \text { and } \\
& \operatorname{ALICE} \Varangle G_{1}(\mathscr{A}, \mathscr{B}) \Longrightarrow \operatorname{ALICE} \Varangle G_{\mathrm{k}}(\mathscr{A}, \mathscr{B}) \Longrightarrow \operatorname{ALICE} \Varangle \mathrm{G}_{\text {fin }}(\mathscr{A}, \mathscr{B})
\end{aligned}
$$

Just like we did with the selection principles, we now focus on some specific instances of the families $\mathscr{A}$ and $\mathscr{B}$ in Definitions 4.0.1, 4.0.4 and 4.0.7.

Exercise 4.0.12. Write the details of Propositions 4.0.6 and 4.0.9's proofs.

\subsection{Covering games}

\subsubsection{The Rothberger game}

We have mentioned in Section 3.3.2 that Question 3.3.10 is closely related to Question 2.3.13 - this relation will begin to get clear in this section. But first let us define a new "level of similarity" between games:

Given a class of spaces $\mathscr{C}$ (like the class of all Hausdorff spaces, for instance), we say two topological games $G_{1}$ and $G_{2}$ are dual over $\mathscr{C}$ if, for every $X \in \mathscr{C}$, both of the following conditions hold: 
- ALICE $\uparrow G_{1}$ on $X$ if, and only if, BoB $\uparrow G_{2}$ on $X$;

- Вов $\uparrow G_{1}$ on $X$ if, and only if, ALICE $\uparrow G_{2}$ on $X$.

If $G_{1}$ and $G_{2}$ are dual over the class of all spaces, we simply say that $G_{1}$ and $G_{2}$ are dual games.

As it turns out, the point-open game and the Rothberger game are dual! In order to show this we use the following lemma.

Lemma 4.1.1. Let $s \in{ }^{<\omega} \mathscr{O}$. If $\sigma$ is a strategy for $\mathrm{BOB}$ in $\mathrm{G}_{1}(\mathscr{O}, \mathscr{O})$ on $X$, then there exists an $x \in X$ such that for every open set $U$ with $x \in U$ there is an open cover $\mathscr{U}$ such that $\sigma\left(s^{\sim} \mathscr{U}\right)=U$.

Proof. Assume not, that is, for all $x \in X$ there is an open set $U_{x}$ with $x \in U_{x}$ such that $\sigma(s \cap \mathscr{U}) \neq$ $U_{x}$ for every open cover $\mathscr{U}$. Let $\mathscr{U}=\left\{U_{x}: x \in X\right\}$. Then clearly $\sigma(s \neg \mathscr{U})=U_{x}$ for some $x \in X$, a contradiction.

Theorem 4.1.2 ([Galvin 1978]). $\mathrm{G}_{1}(\mathscr{O}, \mathscr{O})$ is dual to the point-open game.

Proof. Suppose $\gamma$ is a winning strategy for ALICE in the point-open game. We construct $\sigma$ as the following strategy for B OB in $\mathrm{G}_{1}(\mathscr{O}, \mathscr{O})$ :

- in the first inning, if ALICE chooses the open cover $\mathscr{U}_{0}$, set $\sigma\left(\left\langle\mathscr{U}_{0}\right\rangle\right)=U_{0}$, with $\gamma(\langle\rangle) \in U_{0}$;

- In general (that is, in the inning $n \in \omega)$, set $\sigma\left(\left\langle\mathscr{U}_{0}, \ldots, \mathscr{U}_{n}\right\rangle\right)=U_{n}$, with $\gamma\left(\left\langle U_{0}, \ldots, U_{n-1}\right\rangle\right) \in$ $U_{n}$.

It follows from the fact that $\gamma$ is a winning strategy that $\left\{U_{n}: n \in \omega\right\}$ is not an open cover.

Now, assume that $\gamma$ is a winning strategy for ALICE in $\mathrm{G}_{1}(\mathscr{O}, \mathscr{O})$. Set $\sigma$ as a strategy for Bов in the point-open game as follows:

- if AliCE chooses $x_{0} \in X$ in the first inning, then let $\sigma\left(\left\langle x_{0}\right\rangle\right)=U_{0}$, with $U_{0} \in \gamma(\langle\rangle)$ being such that $x_{0} \in U_{0}$;

- In general, set $\sigma\left(\left\langle x_{0}, \ldots, x_{n}\right\rangle\right)=U_{n}$, with $U_{n} \in \gamma\left(\left\langle U_{0}, \ldots, U_{n-1}\right\rangle\right)$ being such that $x_{n} \in U_{n}$.

It follows from the fact that $\gamma$ is a winning strategy that $\left\{U_{n}: n \in \omega\right\}$ is an open cover.

Now, suppose that $\sigma$ is a winning strategy for Вов in the point-open game. Note that if $s \in{ }^{<\omega} X$, then $\left\{\sigma\left(s^{\wedge} x\right): x \in X\right\}$ is an open cover (in fact, this is true regardless of $\sigma$ being a winning strategy). Then let $\gamma$ be the following strategy for $\operatorname{ALICE}$ in $\mathrm{G}_{1}(\mathscr{O}, \mathscr{O})$ :

- in the first inning, let $\gamma(\langle\rangle)=\{\sigma(\langle x\rangle): x \in X\}$;

- In general, set $\gamma\left(\left\langle U_{0}, \ldots, U_{n}\right\rangle\right)=\left\{\sigma\left(\left\langle x_{0}, \ldots, x_{n}, x\right\rangle\right): x \in X\right\}$ with each $x_{i}$, for $i \leq n$, being such that $\sigma\left(\left\langle x_{0}, \ldots, x_{i}\right\rangle\right)=U_{i}$. 
Since $\sigma$ is a winning strategy, $\left\{U_{n}: n \in \omega\right\}$ does not cover the space and, therefore, $\gamma$ is a winning strategy.

Finally, assume that $\sigma$ is a winning strategy for BOB in $\mathrm{G}_{1}(\mathscr{O}, \mathscr{O})$. We define $\gamma$ as the following strategy for ALICE in the point-open game:

- In the first inning, let $\gamma(\langle\rangle)=x_{0}$ with $x_{0} \in X$ being as in Lemma 4.1.1 for $s=\langle\rangle$;

- In general, set $\gamma\left(\left\langle U_{0}, \ldots, U_{n}\right\rangle\right)=x_{n}$ with $x_{n} \in X$ being as in Lemma 4.1.1 for $s=\left\langle\mathscr{U}_{0}, \ldots, \mathscr{U}_{n-1}\right\rangle$, where $\mathscr{U}_{i}$, for each $i<n$, is the open cover also provided by Lemma 4.1.1 such that $\sigma\left(\left\langle\mathscr{U}_{0}, \ldots, \mathscr{U}_{i}\right\rangle\right)=U_{i}$.

Since $\sigma$ is a winning strategy, $\left\{U_{n}: n \in \omega\right\}$ covers the space and, therefore, $\gamma$ is a winning strategy.

Question 2.3.13 can now be translated to:

Question 4.1.3. Is $\mathrm{G}_{1}(\mathscr{O}, \mathscr{O})$ determined on every subset of $\mathbb{R}$ ?

So the following theorem will later be useful in the search of the answer to Question 4.1.3.

Theorem 4.1.4 ([Telgársky 1983], [Galvin 1978]). Let X be a space in which every point is a $G_{\delta}$ set. Then $\mathrm{Bов} \uparrow \mathrm{G}_{1}(\mathscr{O}, \mathscr{O})$ if, and only if, $X$ is countable.

Proof. If $X$ is countable, then B ОВ obviously has a winning strategy in $\mathrm{G}_{1}(\mathscr{O}, \mathscr{O})$.

So, suppose $\mathrm{Bов} \uparrow \mathrm{G}_{1}(\mathscr{O}, \mathscr{O})$. Then, by Theorem 4.1.2, there is a winning strategy $\gamma$ for ALICE in the point-open game. For each $x \in X$, let $\mathscr{V}_{x}=\left\{V_{n}(x): n \in \omega\right\}$ be such that $\bigcap \mathscr{V}_{x}=\{x\}$ (with a fixed enumeration). We now define, recursively, a countable $\left\{x_{s}: s \in{ }^{<\omega} \omega\right\} \subset X$ and $\left\{V_{s}: s \in{ }^{<\omega} \omega\right\}$, with each $V_{s}$ open, such that, for every $s \in{ }^{<\omega} \omega$,

(a) $x_{s}=\gamma\left(\left\langle V_{s \mid 1}, \ldots, V_{s}\right\rangle\right)$.

(b) $x_{\left(s^{\frown} n\right)^{\wedge} k}=\bigcap_{n \in \omega} V_{s^{\urcorner} n}$.

First, let $x_{\langle\rangle}=\gamma(\langle\rangle)$. Then, for each $n \in \omega$, we let $x_{\langle n\rangle}=\gamma\left(V_{n}\left(x_{\langle\rangle}\right)\right)$. Now, suppose the sets are defined up to $s \in{ }^{<\omega} \omega$. Then we let, for each $n \in \omega$,

$$
\begin{aligned}
& V_{s^{\urcorner} n}=V_{n}\left(x_{s}\right) \text { and } \\
& x_{s^{\urcorner} n}=\gamma\left(\left\langle V_{s\lceil 1}, \ldots, V_{s}, V_{s^{\urcorner} n}\right\rangle\right) .
\end{aligned}
$$

We claim that $X=\left\{x_{s}: s \in{ }^{<\omega} \omega\right\}$. Indeed, suppose there is a $y \in X$ such that $y \notin\left\{x_{s}: s \in{ }^{<\omega} \omega\right\}$. Then Вов may respond to each $x_{s}$ played by $\gamma$ with a $V_{s^{\urcorner} n}$ such that $y \notin V_{s^{\wedge} n}$ (because of property (b)) and will clearly win the game (which contradicts our assumption that $\gamma$ is a winning strategy). 
In view of Theorem 4.1.4, Question 2.3.13 translates to:

Question 4.1.5. Is there an uncountable subspace of the real line on which $\operatorname{ALICE} \Varangle \mathrm{G}_{1}(\mathscr{O}, \mathscr{O})$ ?

This version of Question 2.3.13 will help us relate it with Question 3.3.10 - but we will need to first talk about the Menger game in order to make this connection.

Before moving on to the latter game, it should be noted here that selective games presented in this chapter are all positional, but Вов has no positional winning strategy in most of them. In the case of the Rothberger game, for instance:

Proposition 4.1.6. Вов has a positional winning strategy in $\mathrm{G}_{1}(\mathscr{O}, \mathscr{O})$ on a space $X$ if, and only if, $X \in \mathscr{U}$ for every $\mathscr{U} \in \mathscr{O}$.

Proof. Suppose $X$ has an open cover $\mathscr{U}$ with no unitary subcover. Note that, if $\sigma$ is a positional strategy for Вов in $\mathrm{G}_{1}(\mathscr{O}, \mathscr{O})$, then when AliCE chooses $\mathscr{U}$ in every single inning, $\sigma$ will tell Bов to keep choosing the same open set in every inning. Since $\mathscr{U}$ has no unitary subcover, Bов loses this run, so $\sigma$ is not a winning strategy.

The other implication is obvious.

Exercise 4.1.7. Show that if $\mathrm{BOB} \uparrow \mathrm{G}_{1}(\mathscr{O}, \mathscr{O})$, then $\left(\begin{array}{l}\Omega \\ \Gamma\end{array}\right)$ holds.

Hint: Use Theorems 2.3.14, 2.3.16 and 4.1.2.

\subsubsection{The Menger game}

In general, $\mathrm{G}_{\text {fin }}(\mathscr{A}, \mathscr{B})$ may be quite different from $\mathrm{G}_{1}(\mathscr{A}, \mathscr{B})$. Take the Menger game, for instance:

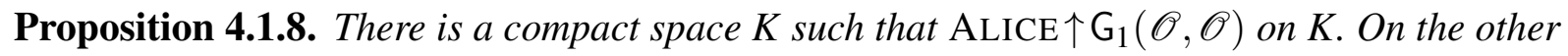
hand, $\mathrm{BoB} \uparrow \mathrm{G}_{\mathrm{fin}}(\mathscr{O}, \mathscr{O})$ on every compact space.

Proof. This follows from Propositions 3.3.7 and 4.0.6.

We then dedicate this section to study the Menger game, starting with its relation with the Menger property $\left(\mathrm{S}_{\text {fin }}(\mathscr{O}, \mathscr{O})\right)$. In view of Proposition 4.0.9, it is already clear that ALICE not having a winning strategy in $\mathrm{G}_{\mathrm{fin}}(\mathscr{O}, \mathscr{O})$ on a given space implies that such space is Menger. This alone shows us the following:

Corollary 4.1.9. Let $X$ be a space. If $\operatorname{ALICE} \nmid \mathrm{G}_{\mathrm{fin}}(\mathscr{O}, \mathscr{O})$, then $X$ is Lindelöf.

As it turns out, however, the converse implication of Proposition 4.0.9 also holds in the Menger case (that is, if $\mathrm{S}_{\text {fin }}(\mathscr{O}, \mathscr{O})$ holds over $X$, then $\operatorname{ALICE}_{X} \mathrm{G}_{\mathrm{fin}}(\mathscr{O}, \mathscr{O})$ over $X$ ). This implication is the deep theorem of Hurewicz. To show it, let us first take a look at an auxiliary game. 
Definition 4.1.10. Given a space $X$, we denote by $\mathscr{O}^{\uparrow}$ the set of all countable and increasing open covers of $X$, that is, $\mathscr{U} \in \mathscr{O}^{\uparrow}$ if $\mathscr{U}=\left\langle U_{n}: n \in \omega\right\rangle$ and $U_{n} \subset U_{n+1}$ for all $n \in \omega$.

Consider the game $\mathrm{G}_{1}\left(\mathscr{O}^{\uparrow}, \mathscr{O}\right)$ on a space $X$, that is, the following game. In each inning $n \in \omega:$

- Alice chooses a countable open cover $\mathscr{U}_{n}=\left\{U_{k}^{n}: k \in \omega\right\}$ such that $U_{k}^{n} \subset U_{k+1}^{n}$;

- Bов chooses $U_{k_{n}}^{n} \in \mathscr{U}_{n}$.

We say that Вов wins if $\bigcup_{n \in \omega} U_{k_{n}}^{n}$ and ALICE wins otherwise. It is worth mentioning that $\mathrm{G}_{1}\left(\mathscr{O}^{\uparrow}, \mathscr{O}\right)$ is very similar to $\mathrm{G}_{\mathrm{fin}}(\mathscr{O}, \mathscr{O})$. The main differences here are that ALICE's choices on $\mathrm{G}_{1}\left(\mathscr{O}^{\uparrow}, \mathscr{O}\right)$ are more restricted than those on $\mathrm{G}_{\mathrm{fin}}(\mathscr{O}, \mathscr{O})$ and BOB may only choose one open set of such covers on $\mathrm{G}_{1}\left(\mathscr{O}^{\uparrow}, \mathscr{O}\right)$ instead of finitely many open sets from ALICE's moves on $\mathrm{G}_{\mathrm{fin}}(\mathscr{O}, \mathscr{O})$. An useful relation rises from the similarities between both games in the proposition that follows.

Proposition 4.1.11. Let $X$ be a Lindelöf space. Then $\mathrm{G}_{1}\left(\mathscr{O}^{\uparrow}, \mathscr{O}\right)$ and $\mathrm{G}_{\mathrm{fin}}(\mathscr{O}, \mathscr{O})$ are equivalent on $X$.

Proof. Let $\gamma$ be a winning strategy for ALICE in $\mathrm{G}_{1}\left(\mathscr{O}^{\uparrow}, \mathscr{O}\right)$. We construct a strategy $\tilde{\gamma}$ for ALICE in $\mathrm{G}_{\mathrm{fin}}(\mathscr{O}, \mathscr{O})$ as follows:

- In the first inning, let $\tilde{\gamma}(\langle\rangle)=\gamma(\langle\rangle)=\left\{U_{0}^{k}: k \in \omega\right\}$, with $U_{0}^{k} \subset U_{0}^{k+1}$ for every $k \in \omega$;

- if Вов responds with a finite $\mathscr{F}_{0} \subset\left\{U_{0}^{k}: k \in \omega\right\}$, let $k_{0}=\max \left\{k \in \omega: U_{0}^{k} \in \mathscr{F}_{0}\right\}$, so that $U_{0}^{k_{0}}=\bigcup \mathscr{F}_{0}$. Then we let $\tilde{\gamma}\left(\left\langle\mathscr{F}_{0}\right\rangle\right)=\gamma\left(\left\langle U_{0}^{k_{0}}\right\rangle\right)=\left\{U_{1}^{k}: k \in \omega\right\}$, with $U_{1}^{k} \subset U_{1}^{k+1}$ for every $k \in \omega$;

- if BoB responds with a finite $\mathscr{F}_{1} \subset\left\{U_{1}^{k}: k \in \omega\right\}$, let $k_{1}=\max \left\{k \in \omega: U_{1}^{k} \in \mathscr{F}_{1}\right\}$, so that $U_{1}^{k_{1}}=\bigcup \mathscr{F}_{1}$. Then we let $\tilde{\gamma}\left(\left\langle\mathscr{F}_{0}, \mathscr{F}_{1}\right\rangle\right)=\gamma\left(\left\langle U_{1}^{k_{1}}\right\rangle\right)=\left\{U_{2}^{k}: k \in \omega\right\}$, with $U_{2}^{k} \subset U_{2}^{k+1}$ for every $k \in \omega$;

- and so on.

Then, clearly, ALICE wins with $\tilde{\gamma}$.

Now, suppose ALICE does not have a winning strategy on $\mathrm{G}_{1}\left(\mathscr{O}^{\uparrow}, \mathscr{O}\right)$ and let $\gamma$ be a strategy for ALICE on $\mathrm{G}_{\text {fin }}(\mathscr{O}, \mathscr{O})$. For each possible cover $\mathscr{U}$ ALICE can choose by $\gamma$, fix $\tilde{\mathscr{U}}=\left\{U_{k}: k \in \omega\right\}$ as one of its countable subcovers and define

$$
\mathscr{U}^{\prime}=\left\{\bigcup_{i \leq k} U_{i}: k \in \omega\right\}
$$


If we replace each of the $\mathscr{U}$ covers from $\gamma$ with the covers $\mathscr{U}^{\prime}$ defined above we get a strategy $\gamma^{\prime}$ for the game $\mathrm{G}_{1}\left(\mathscr{O}^{\uparrow}, \mathscr{O}\right)$, so that $\gamma^{\prime}$ is not a winning strategy.

Let $\mathscr{U}_{0}^{\prime}$ be ALICE's initial move according to $\gamma^{\prime}$ and let $U_{0} \cup \cdots \cup U_{k_{0}}$ be BoB's legal response that will lead him to victory against $\gamma^{\prime}$. Let $\left\{U_{0}, \ldots, U_{k_{0}}\right\}$ be BoB's legal response to $\mathscr{U}_{0}$ in $\mathrm{G}_{\text {fin }}(\mathscr{O}, \mathscr{O})$. Repeating this process for each inning, we find a run of $\mathrm{G}_{\text {fin }}(\mathscr{O}, \mathscr{O})$ compatible with $\gamma$ on which BоB wins, hence, $\gamma$ is not a winning strategy.

The implications for ВОВ will be left as a simple exercise (4.1.24) to the reader.

We now finally present the proof of the Hurewicz Theorem (we will follow the proof presented in [Szewczak and Tsaban 2019]).

Theorem 4.1.12 ([Hurewicz 1926]). If $\mathrm{S}_{\text {fin }}(\mathscr{O}, \mathscr{O})$ holds on a space X, then ALICE does not have a winning strategy in the game $\mathrm{G}_{\mathrm{fin}}(\mathscr{O}, \mathscr{O})$ on $X$.

Proof. Let $X$ be a space satisfying $\mathrm{S}_{\mathrm{fin}}(\mathscr{O}, \mathscr{O})$. Proposition 3.3.2 states that $X$ is Lindelöf, so that by Proposition 4.1.11 it suffices to prove that ALICE does not have a winning strategy in the game $\mathrm{G}_{1}\left(\mathscr{O}^{\uparrow}, \mathscr{O}\right)$.

Let $\gamma$ be a strategy for ALICE on $G_{1}\left(\mathscr{O}^{\uparrow}, \mathscr{O}\right)$. We may assume that, for all $n \in \omega$, if $U$ is BOB's move in the $n$th inning, ALICE's cover $\mathscr{U}=\left\{U_{n}: n \in \omega\right\}$ in the next inning is such that $U_{0}=U$. Indeed, if $U_{0} \neq U$, we can replace $\mathscr{U}$ with the cover $\mathscr{U}^{\prime}=\left\{U, U \cup U_{0}, U \cup U_{1}, \ldots\right\}$ so that if Bов chooses $U$ from $\mathscr{U}^{\prime}$, we provide ALICE with the legal answer $U_{0}$ for $\mathscr{U}$, and if he chooses $U \cup U_{n}$ from $\mathscr{U}^{\prime}$, we provide ALICE with the legal answer $U_{n}$ for $\mathscr{U}$. The addition of $U$ to each of ALICE's open sets does not help BOB cover more points than he already had covered in the previous innings. This way, ALICE will manage to win with her original covers only if she wins with the modified ones presented here.

Now, with these simplifications, ALICE's strategy is identified with the following tree of open sets: ALICE's initial move is an open cover $\left\{U_{\langle n\rangle}: n \in \omega\right\}$. If BoB replies $U_{\langle m\rangle}$, then ALICE's next move is $\left\{U_{\langle m, n\rangle}: n \in \omega\right\}$ with $U_{\langle m, 0\rangle}=U_{\langle m\rangle}$. In general, if BoB replies $U_{s}$, for $s \in{ }^{k} \omega$, then ALICE's next move is an increasing open cover

$$
\mathscr{U}_{s}=\left\{U_{s^{\urcorner} n}: n \in \omega\right\},
$$

with $U_{s^{\urcorner} 0}=U_{s}$. Now we just need to analyze the following concept.

Definition 4.1.13. A countable cover $\mathscr{U}$ of a space $X$ is a tail cover if the set of intersections of cofinite subsets of $\mathscr{U}$ is an open cover of $X$. Equivalently, a cover $\left\{U_{n}: n \in \omega\right\}$ is a tail cover if the family

$$
\left\{\bigcap_{n=0}^{\infty} U_{n}, \bigcap_{n=1}^{\infty} U_{n}, \bigcap_{n=2}^{\infty} U_{n}, \ldots\right\}
$$

of intersections of cofinal segments of the cover is an open cover. 
Claim 4.1.14. Given $n \in \omega$ let $\mathscr{V}_{n}=\bigcup_{s \in{ }^{n} \omega} \mathscr{U}_{s}$. Then the family $\mathscr{V}_{n}$ is a tail cover of $X$.

Proof. The proof is by induction on $n$. Note that the open cover $\mathscr{V}_{0}=\mathscr{U}_{\langle\rangle}$is increasing, so the set of cofinite intersections is also an open cover of X. Let $n \in \omega$. To simplify, enumerate $\mathscr{V}_{n}=\left\{V_{k}: k \in \omega\right\}$, so that

$$
\mathscr{V}_{n+1}=\bigcup_{k \in \omega}\left\{V_{1}^{k}, V_{2}^{k}, \ldots\right\}
$$

where $V_{1}^{k}=V_{k}$ and $V_{i}^{k} \subset V_{i+1}^{k}$ for all $k, i \in \omega$. Assume that the family $\mathscr{V}_{n}$ is a tail cover of $X$. Let $\mathscr{V}$ be a cofinite subset of $\mathscr{V}_{n+1}$ and let

$$
I=\left\{k \in \omega:\left\{V_{1}^{k}, V_{2}^{k}, \ldots\right\} \subset \mathscr{V}\right\}
$$

Note that the set $I$ is a cofinite subset of $\omega$ (otherwise $\mathscr{V}$ would not be cofinite). For each $k \in \omega \backslash I$, let $m_{k}$ be the minimal natural number with $V_{m_{k}}^{k} \in \mathscr{V}$. Then

$$
\bigcap \mathscr{V}=\bigcap_{k \in I}\left(V_{1}^{k} \cap V_{2}^{k} \cap \cdots\right) \cap \bigcap_{k \in \omega \backslash I} \bigcap\left(\left\{V_{1}^{k}, V_{2}^{k}, \ldots\right\} \cap \mathscr{V}\right)=\bigcap_{k \in I} V_{k} \cap \bigcap_{k \in \omega \backslash I} V_{m_{k}}^{n} .
$$

The set $\bigcap_{k \in I} V_{k}$ is an intersection of a cofinite subset of $\mathscr{V}_{n}$, thus it is open. Also, since the set $\omega \backslash I$ is finite, the set $\bigcap_{k \in \omega \backslash I} V_{m_{k}}^{k}$ is open, thus $\bigcap \mathscr{V}$ is open. Let $x \in X$. For nearly all $k \in \omega$, we have $x \in V_{k}$. For the finitely many exceptional numbers $k \in \omega, x$ belongs to almost all sets $V_{m}^{k}: m \in \omega$. Thus, $x$ belongs to all but finitely many members of the family $\mathscr{V}_{n+1}$, or, equivalently, to every intersection of a cofinite subset of $\mathscr{V}_{n+1}$, which concludes our induction.

For each $n \in \omega$, define $\mathscr{V}_{n}^{\prime}$ as the set of intersections of cofinite subsets of $\mathscr{V}_{n}$. Applying the property $\mathrm{S}_{\text {fin }}(\mathscr{O}, \mathscr{O})$ to the sequence $\left\langle\mathscr{V}_{n}^{\prime}: n \in \omega\right\rangle$, we get that for each $n \in \omega$ there is $\mathscr{W}_{n}^{\prime} \subset \mathscr{V}_{n}^{\prime}$ finite such that $X=\bigcup_{n \in \omega} \cup \mathscr{W}_{n}^{\prime}$. Associated with each $\mathscr{W}_{n}^{\prime}$ we have a $\mathscr{W}_{n} \subset \mathscr{V}_{n}$ cofinite so that $X=\bigcup_{n \in \omega} \cap \mathscr{W}_{n}$. In the $n$th inning, ALICE provides BOB with a cover that is an infinite subset of the family $\mathscr{V}_{n}$. Since the family $\mathscr{W}_{n}$ is cofinite in $\mathscr{V}_{n}$, Вов can choose an element $V_{n} \in \mathscr{V}_{n} \cap \mathscr{W}_{n}$. Then $X=\bigcup_{n \in \omega} V_{n}$ and Вов wins, as we wanted to prove.

This characterization of the Menger property with the Menger game will give us some interesting applications in the upcoming sections (for instance, it will help us find examples of $D$-spaces). But, for now, we present a new game that relates to $\mathrm{G}_{\mathrm{fin}}(\mathscr{O}, \mathscr{O})$ similarly to the way the point-open game relate to the Rothberger game. It goes as follows:

Definition 4.1.15. We call the compact-open game the following game on a space $X$. In each inning $n \in \omega$ :

- AliCE chooses $K_{n}$ compact;

- Bов chooses an open set $V_{n} \supset K_{n}$. 
We say that ALICE wins if $X=\bigcup_{n \in \omega} V_{n}$ and BoB wins otherwise.

It is often said that a space $X$ on which ALICE has a winning strategy in the compact-open game is compact-like.

This game then relates to the Menger game in some sort of "semi-duality". In order to better explain this in Theorem 4.1.17, consider the following lemma:

Lemma 4.1.16. Let $\sigma$ be a strategy for $\mathrm{B}$ OB in $\mathrm{G}_{\mathrm{fin}}(\mathscr{O}, \mathscr{O})$ on a regular space $X$. Then, for every $s \in{ }^{<\omega} \mathscr{O}$, the set

$$
K_{s}=\bigcap_{\mathscr{U} \in \mathscr{O}} \overline{\bigcup \sigma(s \cap \mathscr{U})}
$$

is compact.

Proof. Indeed, let $\mathscr{C}$ be an open cover for $K_{s}$ and, for each $x \in K_{s}$, let $U_{x} \in \mathscr{C}$ be such that $x \in U_{x}$. Since $X$ is regular, for every $x \in K_{s}$ there is an open set $V_{x}$ such that $x \in V_{x} \subset \overline{V_{x}} \subset U_{x}$. On the other hand, for each $x \in X \backslash K_{s}$ we consider an open set $V_{x}$ such that $x \in V_{x}$ and $\bar{V}_{x} \cap K_{s}=\emptyset$ (because $K_{s}$ is closed and $X$ is regular). Now, let $\mathscr{U}=\left\{V_{x}: x \in X\right\} \in \mathscr{O}$. In this case, note that

$$
K_{s} \subset \overline{\bigcup \sigma(s \cap \mathscr{U})}
$$

Consider $\mathscr{F}=\left\{V_{x}: x \in K_{s}\right.$ and $\left.V_{x} \in \sigma(s \sim \mathscr{U})\right\}=\left\{V_{x_{i}}: i \leq n\right\}$ with $x_{i} \in K_{s}$ for every $i \leq n$. Then $K_{s} \subset \overline{\cup \mathscr{F}}$. Finally, note that $\left\{U_{x_{i}}: i \leq n\right\}$ is a finite subcover of $\mathscr{C}$.

Theorem 4.1.17 ([Telgársky 1984]). For every space:

(a) If $\mathrm{ALICE}_{\uparrow} \mathrm{G}_{\mathrm{fin}}(\mathscr{O}, \mathscr{O})$, then $\mathrm{B}$ OB has a winning strategy in the compact-open game;

(b) If ALICE has a winning strategy in the compact-open game, then $\mathrm{BoB} \uparrow \mathrm{G}_{\mathrm{fin}}(\mathscr{O}, \mathscr{O})$.

Moreover, if $X$ is a regular space, then $\mathrm{BoB} \uparrow \mathrm{G}_{\text {fin }}(\mathscr{O}, \mathscr{O})$ implies that ALICE has a winning strategy in the compact-open game.

Proof. (a) Let $\gamma$ be a winning strategy for ALICE in $\mathrm{G}_{\text {fin }}(\mathscr{O}, \mathscr{O})$. We then build a winning strategy $\sigma$ for Вов in the compact-open game as follows:

- If AlicE chooses $K_{0}$ in the first inning, let $\mathscr{F}_{0} \subset \gamma(\langle\rangle)$ be a finite cover of $K_{0}$ and then set $\sigma\left(\left\langle K_{0}\right\rangle\right)=\bigcup \mathscr{F}_{0}$;

- if ALICE chooses $K_{1}$ in the next inning, let $\mathscr{F}_{1} \subset \gamma\left(\left\langle\mathscr{F}_{0}\right\rangle\right)$ be a finite cover of $K_{1}$ and then set $\sigma\left(\left\langle K_{0}, K_{1}\right\rangle\right)=\bigcup \mathscr{F}_{1}$;

- if ALICE chooses $K_{2}$ next, let $\mathscr{F}_{2} \subset \gamma\left(\left\langle\mathscr{F}_{0}, \mathscr{F}_{1}\right\rangle\right)$ be a finite cover of $K_{2}$ and then set $\sigma\left(\left\langle K_{0}, K_{1}, K_{2}\right\rangle\right)=\bigcup \mathscr{F}_{2}$;

- and so on. 
Since $\gamma$ is a winning strategy, it is clear that $X \neq \bigcup_{n \in \omega} \sigma\left(\left\langle K_{i}: i \leq n\right\rangle\right)$, so $\sigma$ is a winning strategy.

(b) Let $\gamma$ be a winning strategy for ALICE in the compact-open game. Then we define a winning strategy $\sigma$ for B ОВ in $\mathrm{G}_{\text {fin }}(\mathscr{O}, \mathscr{O})$ as follows:

- If ALICE chooses $\mathscr{U}_{0}$ in the first inning, let $\mathscr{F}_{0} \subset \mathscr{U}_{0}$ be a finite cover of $\gamma(\langle\rangle)$ and then set $\sigma\left(\left\langle\mathscr{U}_{0}\right\rangle\right)=\mathscr{F}_{0}$;

- if in the next inning ALICE chooses $\mathscr{U}_{1}$, let $\mathscr{F}_{1} \subset \mathscr{U}_{1}$ be a finite cover of $\gamma\left(\left\langle\bigcup \mathscr{F}_{0}\right\rangle\right)$ and then set $\sigma\left(\left\langle\mathscr{U}_{0}, \mathscr{U}_{1}\right\rangle\right)=\mathscr{F}_{1}$;

- if ALICE chooses $\mathscr{U}_{2}$ next, let $\mathscr{F}_{2} \subset \mathscr{U}_{2}$ be a finite cover of $\gamma\left(\left\langle\bigcup \mathscr{F}_{0}, \bigcup \mathscr{F}_{1}\right\rangle\right)$ and then set $\sigma\left(\left\langle\mathscr{U}_{0}, \mathscr{U}_{1}, \mathscr{U}_{2}\right\rangle\right)=\mathscr{F}_{2}$;

- and so on.

Since $\gamma$ is a winning strategy, it is clear that $X=\bigcup_{n \in \omega} \sigma\left(\left\langle\mathscr{U}_{i}: i \leq n\right\rangle\right)$. Hence, $\sigma$ is a winning strategy.

Now, suppose $X$ is a regular space and that Bов has a winning strategy $\sigma$ in $\mathrm{G}_{\text {fin }}(\mathscr{O}, \mathscr{O})$ over $X$. Then, by Proposition 4.1.9, $X$ is Lindelöf. The idea here will be to use Lemma 4.1.16 to define a strategy $\gamma$ for ALICE in the compact-open game such that each run $R$ played against $\gamma$ will correspond to a subtree of $\sigma$ in such a way that if a point was not covered in $R$, then we would find a run of such subtree that did not cover this point as well (which is absurd, since we are assuming $\sigma$ is a winning strategy). We remark here that this construction will be possible due to the infinitude of innings and it will appear in the proofs of later theorems again. It goes as follows:

First, let $\gamma(\langle\rangle)=K_{\langle\rangle}$, with $K_{\langle\rangle}$being as in Lemma 4.1.16 and then suppose $V_{0}$ is BoB's response to $\gamma(\langle\rangle)$. Since $\gamma(\langle\rangle) \subset V_{0}$, then $X \backslash V_{0} \subset X \backslash \gamma(\langle\rangle)$, so for each $x \in X \backslash V_{0}$ there is a $\mathscr{U}_{x} \in \mathscr{O}$ such that $x \notin \overline{\bigcup \sigma\left(\left\langle\mathscr{U}_{x}\right\rangle\right)}$ and therefore $\mathscr{C}=\left\{X \backslash \overline{\cup \sigma\left(\left\langle\mathscr{U}_{x}\right\rangle\right)}: x \in X \backslash V_{0}\right\}$ is an open cover for $X \backslash V_{0}$. We have that $X \backslash V_{0}$ is Lindelöf, being a closed subset of a Lindelöf space, then $\mathscr{C}$ must have a countable subcover, so we let, for each $m \in \omega, \mathscr{U}_{\langle m\rangle}$ be such that $\left\{X \backslash \overline{\bigcup \sigma\left(\left\langle\mathscr{U}_{\langle m\rangle}\right\rangle\right)}\right\}$ is an open cover for $X \backslash V_{0}$.

Now, let $\left\{t_{n}: n \in \omega\right\}$ be an enumeration of $\left({ }^{<\omega} \omega\right)^{*}={ }^{<\omega} \omega \backslash\{\langle\rangle\}$ such that $n \leq m$ if $t_{n} \subset t_{m}$ (curious about how such enumeration exists? We leave Exercise 4.1.25 to clarify that out). Immediately, we get:

CLAim 4.1.18. For every $n>0$ there is a $k<n$ and an $m \in \omega$ such that $t_{n}=t_{k}^{\frown} m$.

Proof. Let $n>0$. Let $k \in \omega$ be such that $t_{k}=t_{n} \uparrow\left(\left|t_{n}\right|-1\right)$. Since $t_{k} \subsetneq t_{n}, k<n$. Just let $m=$ $t_{n}\left(\left|t_{n}\right|-1\right)$ and the claim is proved. 
Suppose we defined $\gamma$ up until the inning $n+1$ (with $n \in \omega$ ) and open covers $\mathscr{U}_{t_{\hat{k}} m}$ for all $k \leq n$ and $m \in \omega$, which depend on the open sets played by Bов thus far (the case of the first inning has already been dealt with above). Also, assume we have the following properties:

(1) $\left\{X \backslash \overline{\bigcup \sigma\left(\left\langle\mathscr{U}_{t_{k} \uparrow i}: 0<i \leq\left|t_{k}\right|\right\rangle^{\frown \mathscr{U}_{t_{k} m}}\right)}: m \in \omega\right\}$ covers $X \backslash \bigcup_{j \leq k} V_{k}$ for every $k \leq n$;

(2) $\gamma\left(\left\langle V_{j}: j \leq k\right\rangle\right)=K_{\left\langle\mathscr{U}_{t_{k} \backslash i}: 0<i \leq\left|t_{k}\right|\right\rangle}=\bigcap_{\mathscr{U} \in \mathscr{O}} \overline{\bigcup \sigma\left(\left\langle\mathscr{U}_{t_{k}\lceil i}: 0<i \leq\left|t_{k}\right|\right\rangle^{\curlyvee \mathscr{U}}\right)}$ for every $k \leq n+$ 1.

Note that, by Lemma 4.1.16, $\gamma\left(\left\langle V_{0}, \ldots, V_{j}\right\rangle\right)$ is, indeed, compact.

Since $\gamma\left(\left\langle V_{j}: j \leq n\right\rangle\right) \subset V_{n+1}$, then $X \backslash \bigcup_{j \leq n+1} V_{j} \subset X \backslash \gamma\left(\left\langle V_{j}: j \leq n\right\rangle\right)$, so for each $x \in$ $X \backslash \bigcup_{j \leq n+1} V_{j}$ there is a $\mathscr{U}_{x} \in \mathscr{O}$ such that

$$
x \notin \overline{\bigcup \sigma\left(\left\langle\mathscr{U}_{t_{n}\lceil i}: 0<i \leq\left|t_{n}\right|\right\rangle \frown \mathscr{U}_{x}\right)}
$$

and therefore $\mathscr{C}=\left\{X \backslash \overline{\bigcup \sigma\left(\left\langle\mathscr{U}_{t_{n} \uparrow i}: 0<i \leq\left|t_{n}\right|\right\rangle^{\wedge} \mathscr{U}_{x}\right)}: x \in X \backslash \bigcup_{j \leq n+1} V_{j}\right\}$ is an open cover for $X \backslash \bigcup_{j \leq n+1} V_{j}$. We have that $X \backslash \bigcup_{j \leq n+1} V_{j}$ is Lindelöf, being a closed subset of a Lindelöf space, then $\mathscr{C}$ must have a countable subcover and we may find a $\mathscr{U}_{t_{n}}$ for each $m \in \omega$ such that

$$
\left\{X \backslash \overline{\bigcup \sigma\left(\left\langle\mathscr{U}_{t_{n} \backslash i}: 0<i \leq\left|t_{n}\right|\right\rangle^{\curlyvee} \mathscr{U}_{t_{n} m}\right)}: m \in \omega\right\}
$$

is an open cover for $X \backslash \bigcup_{j \leq n+1} V_{j}$. Hence, condition (1) is satisfied for $k=n+1$.

Now, suppose В ОВ responds with $V_{n+2}$ to $\gamma\left(\left\langle V_{j}: j \leq n+1\right\rangle\right)$ in the inning $n+2$. Note that, in view of Claim $4.1 .18, t_{n+2}=t_{k} m$ for some $k \leq n+1$ and $m \in \omega$, so $\mathscr{U}_{\left(t_{n+2}\right) \uparrow i}$ has already been defined for every $i \leq\left|t_{n+2}\right|$ (according to our recursion hypothesis). Then we may set

$$
\gamma\left(\left\langle V_{j}: j \leq n+2\right\rangle\right)=K_{\left\langle\mathscr{U}_{t_{n+2}}|i: 0<i \leq| t_{n+2} \mid\right\rangle}=\bigcap_{\mathscr{U} \in \mathscr{O}} \overline{\bigcup \sigma\left(\left\langle\mathscr{U}_{t_{n+2}\lceil i}: 0<i \leq\left|t_{n+2}\right|\right\rangle^{\wedge} \mathscr{U}\right)} .
$$

Obviously, $\gamma\left(\left\langle V_{i}: i \leq n+2\right\rangle\right)$ satisfies (2) and again, by Lemma 4.1.16, it is compact. Hence, our recursion is complete

Now that $\gamma$ is well defined with the desired properties, we will show that it is indeed a winning strategy. In order to do that, let

$$
\left\langle\gamma(\langle\rangle), V_{0}, \gamma\left(\left\langle V_{0}\right\rangle\right), V_{1}, \gamma\left(\left\langle V_{0}, V_{1}\right\rangle\right), V_{2} \ldots\right\rangle
$$

be a run compatible with $\gamma$. Then we can recover the tree of open covers $\left\{\mathscr{U}_{s}: s \in\left({ }^{<\omega} \omega\right)^{*}\right\}$ associated to this run that we defined along with $\gamma$. Striving for a contradiction, suppose ALICE loses in this run, that is, that there exists an $x \in X$ such that $x \in X \backslash \bigcup_{n \in \omega} V_{n}$. In particular, $x \in X \backslash V_{0}$ and we can use property (1) to find $i_{0} \in \omega$ such that $x \notin \overline{\bigcup \sigma\left(\left\langle\mathscr{U}_{\left\langle i_{0}\right\rangle}\right\rangle\right)}$. Assume we have found an $i_{j} \in \omega$ for each $j<n$ with $x \notin \overline{\bigcup \sigma\left(\left\langle\mathscr{U}_{\left\langle i_{0}\right\rangle}, \mathscr{U}_{\left\langle i_{0}, i_{1}\right\rangle}, \ldots, \mathscr{U}_{\left\langle i_{0}, \ldots, i_{j}\right\rangle}\right\rangle\right)}$ for all $j<n$. Let $k \in \omega$ be such that $t_{k}=\left\langle i_{j}: j<n\right\rangle$. Then we use again property (1) and the fact that $x \in X \backslash \bigcup_{j \leq k} V_{j}$ to 
obtain $i_{n} \in \omega$ such that $x \notin \overline{\bigcup \sigma\left(\left\langle\mathscr{U}_{\left\langle i_{0}\right\rangle}, \mathscr{U}_{\left\langle i_{0}, i_{1}\right\rangle}, \ldots, \mathscr{U}_{\left\langle i_{0}, \ldots, i_{n}\right\rangle}\right\rangle\right)}$. We have just found a branch in a subtree of $\sigma$ attesting that $\sigma$ is not a winning strategy, a contradiction to our initial assumption. Hence, $\gamma$ is a winning strategy.

As a bonus, we get a result analogous to the one presented in Theorem 4.1.4:

Theorem 4.1.19 ([Telgársky 1983]). Let X be a regular space in which every compact subset is $a G_{\delta}$ set. If $\mathrm{BoB} \uparrow \mathrm{G}_{\mathrm{fin}}(\mathscr{O}, \mathscr{O})$, then $X$ is $\sigma$-compact.

Proof. Analogous to the proof of Theorem 4.1.4 (see Exercise 4.1.26).

Corollary 4.1.20. If $X$ is a regular space with a countable basis such that $\mathrm{BoB} \uparrow \mathrm{G}_{\mathrm{fin}}(\mathscr{O}, \mathscr{O})$, then $X$ is $\sigma$-compact.

Proof. Since $X$ has countable basis and is regular, it is metrizable, which implies that every closed subset of $X$ is a $G_{\delta}$ set (in particular, compact subsets are $G_{\delta}$ sets), so the result follows from Theorem 4.1.19.

One may wonder whether the regularity assumption could be dropped in the last implication of Theorem 4.1.17. To show that this condition is in fact essential, we present the following:

Proposition 4.1.21. There is a Hausdorff space $X$ such that $\mathrm{BoB} \uparrow \mathrm{G}_{\mathrm{fin}}(\mathscr{O}, \mathscr{O})$, but $\mathrm{B}$ oв has a winning strategy in the compact-open game.

Proof. Consider the Cantor set $2^{\omega}$ with its usual topology $\tau$ (which is Hausdorff). Note that Bов $\uparrow \mathrm{G}_{\mathrm{fin}}(\mathscr{O}, \mathscr{O})$, but B Ов has a winning strategy in the point-open game (because, by Proposition 3.3.7, $\mathrm{S}_{1}(\mathscr{O}, \mathscr{O})$ does not hold on $2^{\omega}$, which, by Proposition 4.0.3, implies that $\operatorname{ALICE} \uparrow \mathrm{G}_{1}(\mathscr{O}, \mathscr{O})$, which, by Theorem 4.1.2, implies that Вов has a winning strategy in the point-open game on $2^{\omega}$ ). Then consider a new topology on $2^{\omega}$ that additionally makes every countable set closed, that is, let $\rho$ be the topology generated by

$$
\{U \backslash C: U \in \tau \text { and } C \subset X \text { countable }\}
$$

Note that $\rho$ remains Hausdorff and BOB still has a winning strategy in the point-open game (or, equivalently, the finite-open game) on the new space. Moreover, it is easy to see that $K \subset 2^{\omega}$ is a compact subspace of $\left\langle 2^{\omega}, \rho\right\rangle$ if, and only if, $K$ is finite. So it follows that Вов has a winning strategy in the compact-open game over the modified space.

On the other hand, Вов still has a winning strategy in $\mathrm{G}_{\mathrm{fin}}(\mathscr{O}, \mathscr{O})$ on the new space $\left\langle 2^{\omega}, \rho\right\rangle$. To show this, note that we may assume that AliCE (playing $\mathrm{G}_{\mathrm{fin}}(\mathscr{O}, \mathscr{O})$ on $\left\langle 2^{\omega}, \rho\right\rangle$ ) chooses only covers with basic open sets of the form $U \backslash C$, with $U \in \tau$ and $C$ countable. Given $\mathscr{U}$ open cover of $\left(2^{\omega}, \rho\right)$ with said form we fix, for each $U \in \mathscr{U}, U^{\prime}$ as the open set from the 
original topology such that $U=U^{\prime} \backslash C$ for some $C$ countable. Then we let, for each open cover $\mathscr{U}$ of $(X, \rho)$ with said form,

$$
\mathscr{U}^{\prime}=\left\{U^{\prime} \in \tau: U \in \mathscr{U}\right\} .
$$

Now we define a strategy $\tilde{\sigma}$ as follows:

- in the first inning, if ALICE chooses $\mathscr{U}_{0}$, let $\mathscr{F}_{0} \subset \mathscr{U}_{0}$ be such that $\left\{U^{\prime}: U \in \mathscr{F}_{0}\right\}$ covers $2^{\omega}$ (recall that $2^{\omega}$ is compact with its usual topology) and then set

$$
\tilde{\sigma}\left(\left\langle\mathscr{U}_{0}\right\rangle\right)=\mathscr{F}_{0}
$$

- Note that $\bigcup\left\{U^{\prime}: U \in \mathscr{F}_{0}\right\} \backslash \bigcup \mathscr{F}_{0}$ is countable. Then we let $\tilde{\sigma}$ easily cover these points in the upcoming innings, which concludes the proof.

But it should be noted that we can, actually, find a game that is dual to the compact-open game in a similar fashion to the duality between the point-open game and Rothberger game:

Definition 4.1.22. Given a space $X$, denote by $\mathscr{K}$ the collection of every open $K$-cover of $X$.

Theorem 4.1.23. The compact-open game and $\mathrm{G}_{1}(\mathscr{K}, \mathscr{O})$ are dual.

Proof. Analogous to the proof of Theorem 4.1.2 (see Exercise 4.1.27).

Exercise 4.1.24. Write the details of BoB's implications from Proposition 4.1.11.

Exercise 4.1.25. Fix an enumeration of the prime numbers $\left\{p_{n}: n \in \omega\right\}$. Then, let $\varphi:{ }^{<\omega} \omega \rightarrow \omega$ be such that

$$
\varphi\left(\left\langle n_{j}: j \leq k\right\rangle\right)=\left(\left(\left(p_{n_{0}}\right)^{p_{n_{1}}}\right)^{\cdots}\right)^{p_{n_{k}}} .
$$

Show that $\varphi$ is injective and induces an enumeration of ${ }^{<\omega} \omega$ such that $n \leq m$ if $t_{n} \subset t_{m}$.

Exercise 4.1.26. Write the details of Theorem 4.1.19's proof.

Exercise 4.1.27. Write the details of Theorem 4.1.23's proof.

Exercise 4.1.28. Show that $\mathrm{B}$ ов has a positional winning strategy in $\mathrm{G}_{\mathrm{fin}}(\mathscr{O}, \mathscr{O})$ on a space $X$ if, and only if, $X$ is compact.

Exercise 4.1.29. Given a space $X$, let $G(C, X)$ denote the following game: at first, ALICE chooses a compact $K_{0} \subset X$ and Вов responds with a closed $E_{0} \subset X \backslash K_{0}$. In the inning $n \in \mathbb{N}$ ALICE chooses a compact $K_{n} \subset E_{n-1}$ and В ОВ responds with a closed $E_{n} \subset E_{n-1} \backslash K_{n}$. ALICE wins if $\bigcap_{n \in \omega} E_{n}=\emptyset$ and Вов wins otherwise.

Show that $G(C, X)$ is equivalent to the compact-open game.

Curiosity: the property of being compact-like was first introduced in [Telgársky 1983] with the game $G(C, X)$. 


\subsubsection{The Pawlikowski Theorem}

Our goal in this subsection is to show the Pawlikowski Theorem, which is the $S_{1}$ and $G_{1}$ version of the Hurewicz Theorem 4.1.12 (we will need to invoke the latter a few times in order to do so). But first, consider the following auxiliary game: In each inning $n \in \omega$,

- Alice chooses an open cover $\mathscr{U}_{n}$ for $X$;

- BoB chooses a finite $\mathscr{F}_{n} \subset \mathscr{U}_{n}$.

We say that Вов wins if, for every $m \in \omega, \bigcup_{n \geq m} \mathscr{F}_{n}$ is an open cover for $X$ and ALICE wins otherwise. Since this is a simple modification of $\mathrm{G}_{\text {fin }}(\mathscr{O}, \mathscr{O})$, we will denote this game by $\mathrm{G}_{\text {fin }}^{\prime}(\mathscr{O}, \mathscr{O})$. Then, from Theorem 4.1.12 we have:

Proposition 4.1.30. $\mathrm{G}_{\text {fin }}^{\prime}(\mathscr{O}, \mathscr{O})$ is equivalent to $\mathrm{G}_{\mathrm{fin}}(\mathscr{O}, \mathscr{O})$.

Proof. The implications

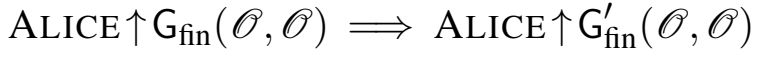

$$
\begin{aligned}
& \text { Вов } \uparrow \mathrm{G}_{\text {fin }}^{\prime}(\mathscr{O}, \mathscr{O}) \Longrightarrow \mathrm{Bов} \uparrow \mathrm{G}_{\text {fin }}(\mathscr{O}, \mathscr{O})
\end{aligned}
$$

are clear.

Now, suppose ALICE $\Varangle \mathrm{G}_{\text {fin }}(\mathscr{O}, \mathscr{O})$ on a space $X$ and let $\gamma$ be a strategy for AlicE in $\mathrm{G}_{\text {fin }}^{\prime}(\mathscr{O}, \mathscr{O})$ on $X$. By Proposition 4.0.9, we know that $\mathrm{S}_{\text {fin }}(\mathscr{O}, \mathscr{O})$ holds on $X$, so $\mathrm{S}_{\text {fin }}(\mathscr{O}, \mathscr{O})$ holds on $X \times \omega$ (considering $\omega$ with the discrete topology).

Let $\pi_{X}: X \times \omega \rightarrow X$ be the projection onto $X$. We now construct a strategy $\tilde{\gamma}$ for ALICE in $\mathrm{G}_{\text {fin }}(\mathscr{O}, \mathscr{O})$ on $X \times \omega$ :

- In the first inning, let $\tilde{\gamma}(\langle\rangle)=\{U \times\{n\}: U \in \gamma(\langle\rangle), n \in \omega\}$;

- If B OB then responds with $\tilde{\mathscr{F}}_{0} \subset \tilde{\gamma}(\langle\rangle)$, let $\mathscr{F}_{0}=\left\{\pi_{X}[U]: U \in \tilde{\mathscr{F}}_{0}\right\}$ and then set $\tilde{\gamma}\left(\left\langle\tilde{\mathscr{F}}_{0}\right\rangle\right)=$ $\left\{U \times\{n\}: U \in \gamma\left(\left\langle\mathscr{F}_{0}\right\rangle\right), n \in \omega\right\}$;

- If in the next inning Bов responds with $\tilde{\mathscr{F}}_{1} \subset \tilde{\gamma}\left(\left\langle\tilde{\mathscr{F}}_{0}\right\rangle\right)$, let $\mathscr{F}_{1}=\left\{\pi_{X}[U]: U \in \tilde{\mathscr{F}}_{1}\right\}$ and then set $\tilde{\gamma}\left(\left\langle\tilde{\mathscr{F}}_{0}, \tilde{\mathscr{F}}_{1}\right\rangle\right)=\left\{U \times\{n\}: U \in \gamma\left(\left\langle\mathscr{F}_{0}, \mathscr{F}_{1}\right\rangle\right), n \in \omega\right\}$

- and so on.

By Theorem 4.1.12, ALICE $\Varangle \mathrm{G}_{\text {fin }}(\mathscr{O}, \mathscr{O})$ over $X \times \omega$, so let $\left\langle\tilde{\mathscr{F}}_{n}: n \in \omega\right\rangle$ be BoB's winning responses to $\tilde{\gamma}$. In order to see that B OB wins in $\mathrm{G}_{\text {fin }}^{\prime}(\mathscr{O}, \mathscr{O})$ with $\left\langle\mathscr{F}_{n}: n \in \omega\right\rangle$, let $x \in X$. By the (Infinite) Pigeonhole Principle, we need infinitely many elements of $\left\{\tilde{\mathscr{F}}_{n}: n \in \omega\right\}$ to cover $\{\langle x, n\rangle: n \in \omega\}$, so infinitely many elements of $\left\{\mathscr{F}_{n}: n \in \omega\right\} \operatorname{cover} x$, as we wanted to prove. 
Finally, suppose there is a winning strategy $\sigma$ for Bов in $\mathrm{G}_{\text {fin }}(\mathscr{O}, \mathscr{O})$. We define a winning strategy $\tilde{\sigma}$ for B ОВ in $\mathrm{G}_{\text {fin }}^{\prime}(\mathscr{O}, \mathscr{O})$ has follows:

$$
\tilde{\sigma}\left(\left\langle\mathscr{U}_{0}, \ldots, \mathscr{U}_{n}\right\rangle\right)=\left\{\begin{array}{l}
\sigma\left(\left\langle\mathscr{U}_{p}, \ldots, \mathscr{U}_{p^{m}}\right\rangle\right) \text { if } n=p^{m} \text { for some } p \text { prime number and } m \geq 1 \\
\sigma\left(\left\langle\mathscr{U}_{0}, \ldots, \mathscr{U}_{n}\right\rangle\right) \text { otherwise (could be anything, actually!). }
\end{array}\right.
$$

Then it is easy to see that $\tilde{\sigma}$ is a winning strategy for BoB in $\mathrm{G}_{\text {fin }}^{\prime}(\mathscr{O}, \mathscr{O})$.

Corollary 4.1.31. If $\mathrm{S}_{\text {fin }}(\mathscr{O}, \mathscr{O})$ holds, then $\operatorname{ALICE} \Varangle \mathrm{G}_{\text {fin }}^{\prime}(\mathscr{O}, \mathscr{O})$.

Proof. This result follows immediately from Theorem 4.1.12 and Proposition 4.1.30.

Now, at once, we can show the Pawlikowski Theorem (again, we will follow the proof presented in [Szewczak and Tsaban 2019]):

Theorem 4.1.32 ([Pawlikowski 1994]). $\mathrm{S}_{1}(\mathscr{O}, \mathscr{O})$ holds on a space $X$ if, and only if, ALICE has no winning strategy in $\mathrm{G}_{1}(\mathscr{O}, \mathscr{O})$ on $X$.

Proof. The implication

$$
\operatorname{ALICE} \nmid G_{1}(\mathscr{O}, \mathscr{O}) \Longrightarrow \mathrm{S}_{1}(\mathscr{O}, \mathscr{O})
$$

follows directly from Proposition 4.0.6.

Now, suppose $\mathrm{S}_{1}(\mathscr{O}, \mathscr{O})$ holds. Then, by Proposition 3.1.9, $\mathrm{S}_{\text {fin }}(\mathscr{O}, \mathscr{O})$ holds, and, by Proposition 3.3.2, $X$ is Lindelöf. With all that in mind, if $\gamma$ is a strategy for ALICE, we may assume that $\gamma$ plays only with countable open covers. Fix an enumeration for each one of $\gamma$ 's covers and this way we may identify $\gamma$ with the tree ${ }^{<\omega} \omega$ as, for each $s \in{ }^{<\omega} \omega$,

$$
\gamma\left(\left\langle U_{s \uparrow 1}, \ldots, U_{s}\right\rangle\right)=\left\{U_{s^{\urcorner} n}: n \in \omega\right\}
$$

Then we define, for each $s \in{ }^{<\omega} \omega, \mathscr{U}_{s}=\gamma\left(\left\langle U_{s \uparrow 1}, \ldots, U_{s}\right\rangle\right)$.

We now define a strategy $\tilde{\gamma}$ for ALICE in $\mathrm{G}_{\text {fin }}^{\prime}(\mathscr{O}, \mathscr{O})$. First, let $\tilde{\gamma}(\langle\rangle)=\mathscr{U}_{\langle\rangle}$. If BoB chooses $\mathscr{F}_{0} \subset \mathscr{U}_{\langle\rangle}$, then let $m_{0}=\max \left\{i_{0}: U_{\left\langle i_{0}\right\rangle} \in \mathscr{F}_{0}\right\}+1$ and

$$
\tilde{\gamma}\left(\left\langle\mathscr{F}_{0}\right\rangle\right)=\bigwedge_{i_{0} \in m_{0}} \mathscr{U}_{\left\langle i_{0}\right\rangle}
$$

If then BOB chooses $\mathscr{F}_{1} \subset \bigwedge_{i_{0} \in m_{0}} \mathscr{U}_{\left\langle i_{0}\right\rangle}$, let $m_{1}=\max \left\{i_{1}: U_{\left\langle i_{0}, i_{1}\right\rangle} \in \mathscr{F}_{1}\right\}+1$ and define

$$
\tilde{\gamma}\left(\left\langle\mathscr{F}_{0}, \mathscr{F}_{1}\right\rangle\right)=\bigwedge_{s \in m_{0} \times m_{1}} \mathscr{U}_{s}
$$

In general, in the inning $n \in \omega$ ALICE plays

$$
\tilde{\gamma}\left(\left\langle\mathscr{F}_{i}: i<n\right\rangle\right)=\bigwedge_{s \in \prod_{i<n} m_{i}} \mathscr{U}_{s}
$$


and, as BоB chooses $\mathscr{F}_{n} \subset \bigwedge_{s \in \prod_{i<n} m_{i}} \mathscr{U}_{s}$, we let $m_{n}=\max \left\{k_{n}: U_{s^{\urcorner} k_{n}} \in \mathscr{F}_{n}\right\}+1$ and then ALICE responds with

$$
\tilde{\gamma}\left(\left\langle\mathscr{F}_{i}: i<n\right\rangle^{\frown \mathscr{F}_{n}}\right)=\bigwedge_{s \in \prod_{i<n+1} m_{i}} \mathscr{U}_{s}
$$

By Corollary 4.1.31, we get that B ОB can win against $\tilde{\gamma}$ if he plays some sequence $\left\langle\mathscr{F}_{n}: n \in \omega\right\rangle$. CLAIM 4.1.33. There are elements $V_{n} \in \mathscr{F}_{n}$, with $n \in \omega$, such that $X=\bigcup_{n \in \omega} V_{n}$.

Proof. For each $k \in \omega$, let $\mathscr{W}_{k}$ be the family of all intersections of $k+1$ open sets taken from different elements from $\left\langle\mathscr{F}_{n}: n \in \omega\right\rangle$. Since $\left\langle\mathscr{F}_{n}: n \in \omega\right\rangle$ is a winning play for BoB in $\mathrm{G}_{\text {fin }}^{\prime}(\mathscr{O}, \mathscr{O})$, each $\mathscr{W}_{k}$ is an open cover of $X$.

Considering $\mathrm{S}_{1}(\mathscr{O}, \mathscr{O})$ holds, we may pick for each $k \in \omega$ an open set $W_{k} \in \mathscr{W}_{k}$ so that $X=\bigcup_{k \in \omega} W_{k}$.

Note that $W_{0} \in \mathscr{F}_{n_{0}}$ for some $n_{0} \in \omega$. Also, we can expand $W_{1}$ to an element of a family $\mathscr{F}_{n_{1}}$ for some $n_{1} \neq n_{0}$ (since $W_{1}$ is the intersection of two elements from two different families). Similarly, we can expand $W_{2}$ to an open set from a family $\mathscr{F}_{n_{2}}$ with $n_{2} \neq n_{0}$ and $n_{2} \neq n_{1}$ (since $W_{2}$ is the intersection of three elements from three different families).

Proceeding in this fashion, we have an open cover from picking at most one open set from each of the elements of $\left\langle\mathscr{F}_{n}: n \in \omega\right\rangle$. Then we pick any open set from the remaining elements (those we did not pick anything from) and this gives us what we wanted.

Now, for each $n \in \omega$, fix a map $\theta_{n}: \prod_{i<n} m_{i} \rightarrow m_{n}$ such that

$$
V_{n}=\bigcap\left\{U_{s\urcorner \theta_{n}(s)}: s \in \prod_{i<n} m_{i}\right\},
$$

and, finally, consider the sequence $f \in{ }^{\omega} \omega$ defined by $f(n)=\theta_{n}(f\lceil n)$ for every $n \in \omega$. Note that $V_{n} \subset U_{f \uparrow(n+1)}$, so the run

$$
\left\langle\mathscr{U}_{\langle\rangle}, U_{f \uparrow 1}, \mathscr{U}_{f \uparrow 1}, U_{f \uparrow 2}, \mathscr{U}_{f \uparrow 2}, \ldots\right\rangle
$$

is compatible with $\gamma$ and is won by Вов.

Now, in view of Theorems 4.1.2, 4.1.4 and 4.1.32, we can finally see that Question 2.3.13 translates to:

Question 4.1.34. Is there an uncountable Rothberger subspace of $\mathbb{R}$ ?

Hence, considering Proposition 3.3.11, a consistent answer to Question 2.3.13 is YES. More precisely: 
Corollary 4.1.35. CH implies the existence of a subset of $\mathbb{R}$ on which the point-open game is undetermined.

But, as previously mentioned, we are actually dealing with an hypothesis independent of ZFC. This will be clear in Section 5.2. For now, our objective is to show, with the help of Theorem 4.1.32, how $\mathrm{G}_{1}(\mathscr{O}, \mathscr{O})$ relates to $\mathrm{G}_{\mathrm{k}}(\mathscr{O}, \mathscr{O})$, with $k \geq 2$. With that in mind, consider the following lemma.

Lemma 4.1.36. Given $k \geq 2$, let $\sigma$ be a strategy for $\mathrm{B}$ ов in $\mathrm{G}_{\mathrm{k}}(\mathscr{O}, \mathscr{O})$ on a Hausdorff space X. Then, for every $s \in{ }^{<\omega} \mathscr{O}$, the set

$$
F_{s}=\bigcap_{\mathscr{U} \in \mathscr{O}} \overline{\bigcup \sigma(s \cap \mathscr{U})}
$$

has at most $k$ points.

Proof. Striving for a contradiction, suppose $x_{0}, \ldots, x_{k}$ are $k+1$ points in $F_{s}$. Since $X$ is Hausdorff, then there are pairwise disjoint open sets $U_{0}, \ldots, U_{k}$ such that $x_{i} \in U_{i}$ for $i \leq k$. Using the fact that $X$ is Hausdorff again, for each $x \in X \backslash\left\{x_{i}: i \leq k\right\}$, let $U_{x}$ be an open set disjoint from an open neighborhood of $\left\{x_{i}: i \leq k\right\}$ such that $x \in U_{x}$. Since $\mathscr{U}=\left\{U_{x}: x \notin\left\{x_{i}: i \leq k\right\}\right\} \cup\left\{U_{i}: i \leq k\right\}$ is an open cover of $X$ and $\sigma\left(s^{\wedge} \mathscr{U}\right)$ is a collection of at most $k$ open sets of $\mathscr{U}$, we conclude that there must be an $i \leq k$ such that $U_{i} \notin \sigma(\langle\mathscr{U}\rangle)$, which contradicts the assumption that $x_{i} \in \gamma(\langle\rangle)$.

Theorem 4.1.37 ([Crone et al. 2019]). Let X be a space and $k \geq 2$. Then the following properties are equivalent:

(A1) $\operatorname{ALICE} \uparrow G_{1}(\mathscr{O}, \mathscr{O})$;

(A2) $\operatorname{ALICE} \uparrow \mathrm{G}_{\mathrm{k}}(\mathscr{O}, \mathscr{O})$.

Moreover, if $X$ is Hausdorff, then the following properties are also equivalent:

(B1) $\mathrm{B} \mathrm{OB} \uparrow \mathrm{G}_{1}(\mathscr{O}, \mathscr{O})$;

(B2) $\mathrm{BoB} \uparrow \mathrm{G}_{\mathrm{k}}(\mathscr{O}, \mathscr{O})$.

Proof. The implications

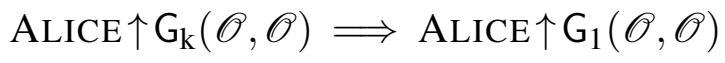

$$
\begin{aligned}
& \mathrm{BOB} \uparrow \mathrm{G}_{1}(\mathscr{O}, \mathscr{O}) \Longrightarrow \mathrm{B} \mathrm{ов} \uparrow \mathrm{G}_{\mathrm{k}}(\mathscr{O}, \mathscr{O})
\end{aligned}
$$

are clear.

Now, assume ALICE has a winning strategy in $\mathrm{G}_{1}(\mathscr{O}, \mathscr{O})$. Then, by Theorem 4.1.32, there exists a sequence $\left\langle\mathscr{U}_{n}: n \in \omega\right\rangle$ of open covers such that for every sequence $\left\langle U_{n}: n \in \omega\right\rangle$ with $U_{n} \in \mathscr{U}_{n}, X \neq \bigcup_{n \in \omega} U_{n}$. We define a winning strategy for ALICE in $\mathrm{G}_{\mathrm{k}}(\mathscr{O}, \mathscr{O})$ as follows: 
- in the first inning, let

$$
\gamma(\langle\rangle)=\bigwedge_{i<k} \mathscr{U}_{i}
$$

- if BoB chooses

$$
\mathscr{F}_{0}=\left\{\bigcap_{i<k} V_{j}^{i}: j<k\right\},
$$

with $V_{j}^{i} \in \mathscr{U}_{i}$, set

$$
\gamma\left(\left\langle\mathscr{F}_{0}\right\rangle\right)=\bigwedge_{k \leq i<2 k} \mathscr{U}_{i}
$$

- In general (that is, in the inning $n+1$ ), if Вов chose

$$
\mathscr{F}_{n}=\left\{\bigcap_{n k \leq i<(n+1) k} V_{j}^{i}: j<k\right\},
$$

in the inning $n \in \omega$, set

$$
\gamma\left(\left\langle\mathscr{F}_{j}: j \leq n\right\rangle\right)=\bigwedge_{(n+1) k \leq i<(n+2) k} \mathscr{U}_{i}
$$

To see that this is a winning strategy, suppose a run $\left\langle\mathscr{F}_{n}: n \in \omega\right\rangle$ is played by BOB against $\gamma$. Let $U_{n}=V_{n}^{n} \in \mathscr{U}_{n}$ for each $n \in \omega$. Then $\bigcup_{n \in \omega} U_{n} \supset \bigcup_{n \in \omega} \bigcup \mathscr{F}_{n}$. Hence, considering that $\left\langle U_{n}: n \in \omega\right\rangle$ must not cover $X, \gamma$ is a winning strategy.

Now, suppose $X$ is a Hausdorff space and that B ОВ has a winning strategy $\sigma$ in $\mathrm{G}_{\mathrm{k}}(\mathscr{O}, \mathscr{O})$ over $X$. Then, by Propositions 4.0.3 and 3.3.2, $X$ is Lindelöf. We will now define a strategy $\gamma$ for ALICE in the finite-open game in a way similar to what we did in the proof of Theorem 4.1.17's last implication:

First, let $\gamma(\langle\rangle)=F_{\langle\rangle}$, with $F_{\langle\rangle}$being as in Lemma 4.1.36 and then suppose $V_{0}$ is BoB's response to $\gamma(\langle\rangle)$. Since $\gamma(\langle\rangle) \subset V_{0}$, then $X \backslash V_{0} \subset X \backslash \gamma(\langle\rangle)$, so for each $x \in X \backslash V_{0}$ there is a $\mathscr{U}_{x} \in \mathscr{O}$ such that $x \notin \overline{\cup \sigma\left(\left\langle\mathscr{U}_{x}\right\rangle\right)}$ and therefore $\mathscr{C}=\left\{X \backslash \overline{\cup \sigma\left(\left\langle\mathscr{U}_{x}\right\rangle\right)}: x \in X \backslash V_{0}\right\}$ is an open cover for $X \backslash V_{0}$. We have that $X \backslash V_{0}$ is Lindelöf, being a closed subset of a Lindelöf space, then $\mathscr{C}$ must have a countable subcover, so we let, for each $m \in \omega, \mathscr{U}_{\langle m\rangle}$ be such that $\left\{X \backslash \overline{U \sigma\left(\left\langle\mathscr{U}_{\langle m\rangle}\right\rangle\right)}\right\}$ is an open cover for $X \backslash V_{0}$.

Now, let $\left\{t_{n}: n \in \omega\right\}$ be an enumeration of $\left({ }^{<\omega} \omega\right)^{*}={ }^{<\omega} \omega \backslash\{\langle\rangle\}$ such that $n \leq m$ if $t_{n} \subset t_{m}$. In this case, recall that:

CLAim 4.1.38. For every $n>0$ there is a $k<n$ and an $m \in \omega$ such that $t_{n}=t_{k} m$.

Suppose we defined $\gamma$ up until the inning $n+1$ (with $n \in \omega$ ) and open covers $\mathscr{U}_{t_{k} m}$ for all $k \leq n$ and $m \in \omega$, which depend on the open sets played by Вов thus far (the case of the first inning has already been dealt with above). Also, assume we have the following properties:

(1) $\left\{X \backslash \overline{\bigcup \sigma\left(\left\langle\mathscr{U}_{t_{k} \uparrow i}: 0<i \leq\left|t_{k}\right|\right\rangle^{\frown \mathscr{U}_{t_{k} m}}\right)}: m \in \omega\right\}$ covers $X \backslash \bigcup_{j \leq k} V_{j}$ for every $k \leq n$; 
(2) $\left.\gamma\left(\left\langle V_{j}: j \leq k\right\rangle\right)=F_{\left\langle\mathscr{U}_{t_{k}} \backslash i\right.}: 0<i \leq\left|t_{k}\right|\right\rangle=\bigcap_{\mathscr{U} \in \mathscr{O}} \overline{\left.\bigcup \sigma\left(\left\langle\mathscr{U}_{t_{k} \backslash i}: 0<i \leq\left|t_{k}\right|\right\rangle\right\urcorner \mathscr{U}\right)}$ for every $k \leq n+$ 1.

Note that, by Lemma 4.1.36, $\gamma\left(\left\langle V_{0}, \ldots, V_{j}\right\rangle\right)$ is, indeed, finite.

Since $\gamma\left(\left\langle V_{j}: j \leq n\right\rangle\right) \subset V_{n+1}$, then $X \backslash \bigcup_{j \leq n+1} V_{j} \subset X \backslash \gamma\left(\left\langle V_{j}: j \leq n\right\rangle\right)$, so for each $x \in$ $X \backslash \bigcup_{j \leq n+1} V_{j}$ there is a $\mathscr{U}_{x} \in \mathscr{O}$ such that

$$
x \notin \overline{\bigcup \sigma\left(\left\langle\mathscr{U}_{t_{n} \uparrow i}: 0<i \leq\left|t_{n}\right|\right\rangle \mathscr{U}_{x}\right)}
$$

and therefore $\mathscr{C}=\left\{X \backslash \overline{\bigcup \sigma\left(\left\langle\mathscr{U}_{t_{n} \uparrow i}: 0<i \leq\left|t_{n}\right|\right\rangle^{\wedge} \mathscr{U}_{x}\right)}: x \in X \backslash \bigcup_{j \leq n+1} V_{j}\right\}$ is an open cover for $X \backslash \bigcup_{j \leq n+1} V_{j}$. We have that $X \backslash \bigcup_{j \leq n+1} V_{j}$ is Lindelöf, being a closed subset of a Lindelöf space, then $\mathscr{C}$ must have a countable subcover and we may find a $\mathscr{U}_{t_{n} m}$ for each $m \in \omega$ such that

$$
\left\{X \backslash \overline{\bigcup \sigma\left(\left\langle\mathscr{U}_{t_{n} \uparrow i}: 0<i \leq\left|t_{n}\right|\right\rangle^{\frown \mathscr{U}_{t_{n} m}}\right)}: m \in \omega\right\}
$$

is an open cover for $X \backslash \bigcup_{j \leq n+1} V_{j}$. Hence, condition (1) is satisfied for $k=n+1$.

Now, suppose Вов responds with $V_{n+2}$ to $\gamma\left(\left\langle V_{j}: j \leq n+1\right\rangle\right)$ in the inning $n+2$. Note that, in view of Claim 4.1.38, $t_{n+2}=t_{k}^{\urcorner} m$ for some $k \leq n+1$ and $m \in \omega$, so $\mathscr{U}_{\left(t_{n+2}\right) \uparrow i}$ has already been defined for every $i \leq\left|t_{n+2}\right|$ (according to our recursion hypothesis). Then we may set

$$
\gamma\left(\left\langle V_{j}: j \leq n+2\right\rangle\right)=F_{\left\langle\mathscr{U}_{t_{n+2} \mid i}: 0<i \leq\left|t_{n+2}\right|\right\rangle}=\bigcap_{\mathscr{U} \in \mathscr{O}} \overline{\bigcup \sigma\left(\left\langle\mathscr{U}_{t_{n+2}\lceil i}: 0<i \leq\left|t_{n+2}\right|\right\rangle^{\curlyvee} \mathscr{U}\right)} .
$$

Obviously, $\gamma\left(\left\langle V_{i}: i \leq n+2\right\rangle\right)$ satisfies (2) and again, by Lemma 4.1.36, it is finite. Hence, our recursion is complete

Now that $\gamma$ is well defined with the desired properties, we will show that it is indeed a winning strategy. In order to do that, let

$$
\left\langle\gamma(\langle\rangle), V_{0}, \gamma\left(\left\langle V_{0}\right\rangle\right), V_{1}, \gamma\left(\left\langle V_{0}, V_{1}\right\rangle\right), V_{2} \ldots\right\rangle
$$

be a run compatible with $\gamma$. Then we can recover the tree of open covers $\left\{\mathscr{U}_{s}: s \in\left({ }^{<\omega} \omega\right)^{*}\right\}$ associated to this run that we defined along with $\gamma$. Striving for a contradiction, suppose ALICE loses in this run, that is, that there exists an $x \in X$ such that $x \in X \backslash \bigcup_{n \in \omega} V_{n}$. In particular, $x \in X \backslash V_{0}$ and we can use property (1) to find $i_{0} \in \omega$ such that $x \notin \overline{\cup \sigma\left(\left\langle\mathscr{U}_{\left\langle i_{0}\right\rangle}\right\rangle\right)}$. Assume we have found an $i_{j} \in \omega$ for each $j<n$ with $x \notin \overline{\bigcup \sigma\left(\left\langle\mathscr{U}_{\left\langle i_{0}\right\rangle}, \mathscr{U}_{\left\langle i_{0}, i_{1}\right\rangle}, \ldots, \mathscr{U}_{\left\langle i_{0}, \ldots, i_{j}\right\rangle}\right\rangle\right)}$ for all $j<n$. Let $k \in \omega$ be such that $t_{k}=\left\langle i_{j}: j<n\right\rangle$. Then we use again property (1) and the fact that $x \in X \backslash \bigcup_{j \leq k} V_{j}$ to obtain $i_{n} \in \omega$ such that $x \notin \overline{\bigcup \sigma\left(\left\langle\mathscr{U}_{\left\langle i_{0}\right\rangle}, \mathscr{U}_{\left\langle i_{0}, i_{1}\right\rangle}, \ldots, \mathscr{U}_{\left\langle i_{0}, \ldots, i_{n}\right\rangle}\right\rangle\right)}$. We have just found a branch in a subtree of $\sigma$ witnessing that $\sigma$ is not a winning strategy, a contradiction to our initial assumption. Hence, $\gamma$ is a winning strategy.

Corollary 4.1.39. Let $k \in \omega$. Then $\mathrm{G}_{1}(\mathscr{O}, \mathscr{O})$ is equivalent to $\mathrm{G}_{\mathrm{k}}(\mathscr{O}, \mathscr{O})$ over the class of Hausdorff spaces.

Exercise 4.1.40. Complete the proof of Proposition 4.1.11. 


\subsubsection{The Hurewicz game}

We can also characterize Hurewicz spaces in terms of a topological game. This game goes as follows:

Definition 4.1.41. We call the Hurewicz game the game on a space $X$ in which for each inning $n \in \omega:$

- ALICE chooses an open cover $\mathscr{U}_{n}$ for $X$;

- BoB chooses a finite $\mathscr{F}_{n} \subset \mathscr{U}_{n}$.

We then say that B OB wins if $X=\bigcup_{n \in \omega} \bigcap_{k \geq n}\left(\bigcup \mathscr{F}_{k}\right)$, as ALICE wins otherwise.

Theorem 4.1.42 ([Scheepers 1996]). Property $\cup_{\text {fin }}(\mathscr{O}, \Gamma)$ holds on a space $X$ if, and only if, ALICE has no winning strategy in the Hurewicz game.

Proof. Suppose $X$ is a Hurewicz space and let $\gamma$ be a strategy for ALICE in the Hurewicz game (note that, since $X$ is Hurewicz, we may assume that $\gamma$ only plays with countable covers). We associate to each $t \in{ }^{<\omega} \omega \backslash\{\emptyset\}$ an open set $U_{t}$ with the following recursion. First, define $U_{\langle k\rangle}$ in such a way that $\gamma(\langle\rangle)=\left\{U_{\langle k\rangle}: k \in \omega\right\}$. Now, suppose $U_{t}$ is defined for all $t \in{ }^{<\omega} \omega \backslash\{\emptyset\}$ such that $|t| \leq n+1$ and let $s \in{ }^{<\omega} \omega \backslash\{\emptyset\}$ be such that $|s|=n+1$. Then we define $U_{s^{\urcorner} k}$ in such a way that

$$
\gamma\left(\left\langle\left\{U_{\langle k\rangle}: k \leq s(0)\right\}, \ldots,\left\{U_{\left(s\lceil n-1)^{\wedge} k\right.}: k \leq s(n)\right\}\right\rangle\right)=\left\{U_{s^{\prime} k}: k \in \omega\right\} .
$$

Since $X$ is Hurewicz, we can find for every $t \in{ }^{<\omega} \omega$ an $m_{t} \in \omega$ such that by letting $\mathscr{F}_{t}=$ $\left\{U_{t^{\frown} k}: k \leq m_{t}\right\}$, each point of the space is in all but finitely many of the open sets of the open cover $\left\{\bigcup \mathscr{F}_{t}: t \in{ }^{<\omega} \omega\right\}$. Now, consider the sequence $\left\langle k_{n}: n \in \omega\right\rangle$ recursively defined as follows:

$$
k_{n}=m_{\left\langle k_{l}: l<n\right\rangle} .
$$

Clearly, В ОВ wins against $\gamma$ by playing in each inning $n \in \omega$ with $\left\{U_{\left\langle k_{l}: l<n\right\rangle^{\urcorner} k}: k \leq k_{n}\right\}$, hence $\gamma$ is not a winning strategy.

We let the other implication as an exercise to the reader (see 4.1.43).

Exercise 4.1.43. Show that if ALICE has no winning strategy in the Hurewicz game on a space $X$, then $\mathrm{U}_{\text {fin }}(\mathscr{O}, \Gamma)$ holds over $X$.

\subsubsection{The Alster game}

Definition 4.1.44. Given a space $X$, the Alster game is the game denoted by $\mathrm{G}_{1}\left(\mathscr{K}_{\delta}, \mathscr{O}_{\delta}\right)$, that is, the game in which, in each inning $n \in \omega$, 
- ALICE chooses an Alster cover $\mathscr{U}_{n}$;

- BoB responds with $G_{n} \in \mathscr{U}_{n}$,

BoB wins if $\bigcup_{n \in \omega} G_{n}=X$ and ALICE wins otherwise.

Immediately, we get:

Proposition 4.1.45. If ALICE $\Varangle G_{1}\left(\mathscr{K}_{\delta}, \mathscr{O}_{\delta}\right)$ on a space $X$, then $X$ is Alster.

Proof. This follows directly from Propositions 4.0.3 and 3.3.30.

Whether the inverse implication of Proposition 4.1.45 holds (like for Rothberger or Menger spaces) or not, it remains unknown. But we can find a duality analogous to the one between $\mathrm{G}_{1}(\mathscr{O}, \mathscr{O})$ and the point-open game:

Definition 4.1.46. Given a space $X$, the compact- $G_{\delta}$ game is the game in which, in each inning $n \in \omega$,

- AliCE chooses a compact $K_{n} \subset X$;

- BoB responds with a $G_{\delta} G_{n} \supset K_{n}$,

AlICE wins if $\bigcup_{n \in \omega} G_{n}=X$ and Вов wins otherwise.

Theorem 4.1.47. The Alster and the compact- $G_{\delta}$ games are dual.

What is surprising, though, is that, for ALICE, having a winning strategy in the compact$G_{\delta}$ game or in the compact-open game makes no difference:

Theorem 4.1.48 ([Telgársky 1983]). On every space X, ALICE has a winning strategy in the compact-open game if, and only if, ALICE has a winning strategy in the compact-G $G_{\delta}$ game.

Proof. Clearly, if ALICE has a winning strategy $\gamma$ in the compact- $G_{\delta}$ game, then a restriction of $\gamma$ works as a winning strategy in the compact-open game.

So, suppose $\gamma$ is a winning strategy for ALICE in the compact-open game. We define a winning strategy $\tilde{\gamma}$ for ALICE in the compact- $G_{\delta}$, again, using some ideas from the proof of the last implication of Theorem 4.1.23:

First, set $\tilde{\gamma}(\langle\rangle)=\gamma(\langle\rangle)$. If Вов then responds with a $G_{\delta}$ set $G_{0} \supset \tilde{\gamma}(\langle\rangle)$, let $\left\{V_{\langle m\rangle}: m \in \omega\right\}$ be such that each $V_{\langle m\rangle}$ is open and $G_{0}=\bigcap_{m \in \omega} V_{\langle m\rangle}$.

Now, let $\left\{t_{n}: n \in \omega\right\}$ be an enumeration of $\left({ }^{<\omega} \omega\right)^{*}={ }^{<\omega} \omega \backslash\{\langle\rangle\}$ such that $n \leq m$ if $t_{n} \subset t_{m}$. Once again, recall that:

CLAIM 4.1.49. For every $n>0$ there is a $k<n$ and an $m \in \omega$ such that $t_{n}=t_{k}^{\frown} m$. 
Suppose we defined $\tilde{\gamma}$ up until the inning $n+1$ (with $n \in \omega$ ) and open sets $V_{t_{\bar{k}} m}$ for all $k \leq n$ and $m \in \omega$, which depend on the $G_{\delta}$ sets played by Вов thus far (the case of the first inning has already been dealt with above). Also, assume we have the following properties:

(1) $G_{k}=\bigcap_{m \in \omega} V_{t_{k} m}$ for every $k \leq n$;

(2) $\tilde{\gamma}\left(\left\langle G_{j}: j \leq k\right\rangle\right)=\gamma\left(\left\langle V_{t_{k} \uparrow i}: 0<i \leq\left|t_{k}\right|\right\rangle\right)$ for every $k \leq n+1$.

Considering $G_{n+1}$ is a $G_{\delta}$ set, we may find for each $m \in \omega$ an open $V_{t_{n} m}$ such that

$$
G_{n+1}=\bigcap_{m \in \omega} V_{t_{n} m}
$$

Hence, condition (1) is satisfied for $k=n+1$.

Now, suppose Вов responds with $G_{n+2}$ to $\tilde{\gamma}\left(\left\langle G_{j}: j \leq n+1\right\rangle\right)$ in the inning $n+2$. Note that, in view of Claim 4.1.49, $t_{n+2}=t_{k}^{\frown} m$ for some $k \leq n+1$ and $m \in \omega$, so $V_{\left(t_{n+2}\right) \vdash i}$ has already been defined for every $0<i \leq\left|t_{n+2}\right|$ (according to our recursion hypothesis). Then we may set

$$
\tilde{\gamma}\left(\left\langle G_{j}: j \leq n+2\right\rangle\right)=\gamma\left(\left\langle V_{t_{n+2}\lceil i}: 0<i \leq\left|t_{n+2}\right|\right\rangle\right) .
$$

Obviously, $\tilde{\gamma}\left(\left\langle G_{i}: i \leq n+2\right\rangle\right)$ satisfies (2) for $k=n+2$ and, hence, our recursion is complete.

Now that $\tilde{\gamma}$ is well defined with the desired properties, we will show that it is indeed a winning strategy. In order to do that, let

$$
\left\langle\tilde{\gamma}(\langle\rangle), G_{0}, \tilde{\gamma}\left(\left\langle G_{0}\right\rangle\right), G_{1}, \tilde{\gamma}\left(\left\langle G_{0}, G_{1}\right\rangle\right), G_{2} \ldots\right\rangle
$$

be a run compatible with $\tilde{\gamma}$. Then we can recover the tree of open sets $\left\{V_{s}: s \in\left({ }^{<\omega} \omega\right)^{*}\right\}$ associated to this run that we defined along with $\tilde{\gamma}$. Striving for a contradiction, suppose ALICE loses in this run, that is, that there exists an $x \in X$ such that $x \in X \bigcup_{n \in \omega} G_{n}$. In particular, $x \in X \backslash G_{0}$ and we can use property (1) to find $i_{0} \in \omega$ such that $x \notin V_{\left\langle i_{0}\right\rangle}$. Assume we have found an $i_{j} \in \omega$ for each $j<n$ with $x \notin V_{\left\langle i_{0}, \ldots, i_{j}\right\rangle}$ for all $j<n$. Let $k \in \omega$ be such that $t_{k}=\left\langle i_{j}: j<n\right\rangle$. Then we use again property (1) and the fact that $x \in X \backslash \bigcup_{j \leq k} G_{j}$ to obtain $i_{n} \in \omega$ such that $x \notin V_{\left\langle i_{0}, \ldots, i_{n}\right\rangle}$. We have just found a branch in a subtree of $\gamma$ attesting that $\gamma$ is not a winning strategy, a contradiction to our initial assumption. Hence, $\tilde{\gamma}$ is a winning strategy.

Corollary 4.1.50. If $X$ is a regular space, then the following properties are equivalent:

(a) $\operatorname{BoB} \uparrow \mathrm{G}_{\text {fin }}(\mathscr{O}, \mathscr{O})$;

(b) ALICE has a winning strategy in the compact-open game;

(c) ALICE has a winning strategy in the compact-G $G_{\delta}$ game; 
(d) $\operatorname{BoB} \uparrow G_{1}\left(\mathscr{A}, \mathscr{O}_{\delta}\right)$.

Proof. (a) is equivalent to (b) by Theorem 4.1.23 (we use regularity here), (b) is equivalent to (c) by Theorem 4.1.48 and (c) is equivalent to (d) by Theorem 4.1.47.

Corollary 4.1.51. If $X$ is a compact-like space, then $X$ is an Alster space.

Corollary 4.1.52. If $X$ is a regular space such that $\mathrm{BoB} \uparrow \mathrm{G}_{\text {fin }}(\mathscr{O}, \mathscr{O})$, then $X$ is an Alster space.

Proof. This follows from Theorem 4.1.23 and Corollary 4.1.51.

\subsection{Closure games}

We have discussed a bit of closure-related selection principles in Section 3.2 - so it only makes sense to take a look at its game-counterparts, so given a space $X$ and $x \in X$, recall that $\Omega_{x}$ denote the family of all subsets of $X$ that contains $x$ in its closure. We can therefore define the games $\mathrm{G}_{1}\left(\Omega_{x}, \Omega_{x}\right), \mathrm{G}_{\mathrm{k}}\left(\Omega_{x}, \Omega_{x}\right)$ and $\mathrm{G}_{\mathrm{fin}}\left(\Omega_{x}, \Omega_{x}\right)$ :

Example 4.2.1. We remind the reader that $\mathrm{G}_{1}\left(\Omega_{x}, \Omega_{x}\right)$ denotes the game in which in each inning $n \in \omega$, Alice chooses $A_{n} \in \Omega_{x}$ so that Вов responds with $x_{n} \in A_{n}$, and ВоB wins if $\left\langle x_{n}: n \in \omega\right\rangle$ has $x$ in its closure (ALICE wins otherwise).

Example 4.2.2. Given $k \geq 2$, recall that $\mathrm{G}_{\mathrm{k}}\left(\Omega_{x}, \Omega_{x}\right)$ denotes the game in which in each inning $n \in \omega$,ALICE chooses $A_{n} \in \Omega_{x}$ so that B OB responds with $F_{n} \subset A_{n}$ with at most $k$ points, and BoB wins if $\bigcup_{n \in \omega} F_{n}$ has $x$ in its closure (ALICE wins otherwise).

Example 4.2.3. Recall that $\mathrm{G}_{\text {fin }}\left(\Omega_{x}, \Omega_{x}\right)$ denotes the game in which in each inning $n \in \omega$, ALICE chooses $A_{n} \in \Omega_{x}$ so that $\mathrm{BOB}$ responds with a finite $F_{n} \subset A_{n}$, and BoB wins if $\bigcup_{n \in \omega} F_{n}$ has $x$ in its closure (ALICE wins otherwise).

Examples 4.2.1, 4.2.2 and 4.2.3 are called tightness games.

For now, we will be focusing on $\mathrm{G}_{\mathrm{k}}\left(\Omega_{x}, \Omega_{x}\right)$ and $\mathrm{G}_{1}\left(\Omega_{x}, \Omega_{x}\right)$. In Section 3.2 we had shown that $\mathrm{S}_{1}\left(\Omega_{x}, \Omega_{x}\right)$ and $\mathrm{S}_{\mathrm{k}}\left(\Omega_{x}, \Omega_{x}\right)$ are the same property. Remarkably, the same thing cannot be said about their respective games. To show this, consider the following example:

Example 4.2.4 ([Scheepers 1997]). Consider ${ }^{<\omega} \omega$ with the discrete topology and let $X=$ ${ }^{<\omega} \omega \cup\{p\}$ with open neighborhoods of $p$ being the entire space, except for finitely many branches of ${ }^{<\omega} \omega$. We then have:

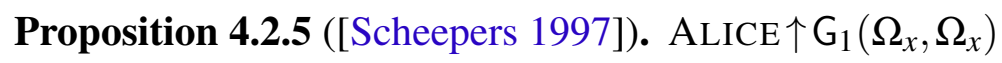

Proof. Consider the following strategy $\gamma$ for ALICE: 
- In the first inning, let $\gamma(\langle\rangle)=\{\langle n\rangle: n \in \omega\}$;

- if Bов chooses $\left\langle m_{0}\right\rangle$, then let $\left.\gamma\left(\left\langle\left\langle m_{0}\right\rangle\right\rangle\right)=\left\{\left\langle m_{0}\right\rangle\right\urcorner n: n \in \omega\right\}$ in the next inning;

- if BoB chooses $\left\langle m_{0}, m_{1}\right\rangle$, then let $\gamma\left(\left\langle m_{0}, m_{1}\right\rangle\right)=\left\{\left\langle m_{0}, m_{1}\right\rangle^{\urcorner} n: n \in \omega\right\}$ next;

- and so on.

In this case, the collection of BOB's moves will be a branch of ${ }^{<\omega} \omega$ and clearly $x$ is not be in the closure of a single branch.

Proposition 4.2.6 ([7]). Вов $\uparrow \mathrm{G}_{2}\left(\Omega_{x}, \Omega_{x}\right)$

Proof. We will consider that ALICE chooses only subsets of ${ }^{<\omega} \omega$ (otherwise, if ALICE chooses a subset containing $p$, then Вов can choose $p$ and trivially win).

In this case, consider the following strategy $\sigma$ for Вов:

- If in the first inning ALICE chooses $A_{0} \subset{ }^{<\omega} \omega$, then (since $x$ is in the closure of $A_{0}$ ) there must be $s_{1}, s_{2} \in A$ such that $s_{1} \perp s_{2}$, so let $\sigma\left(\left\langle A_{0}\right\rangle\right)=F_{0}=\left\{s_{1}, s_{2}\right\}$;

- We say that $\sigma$ can be constructed in such a way that for every inning $n \in \omega$, there will be $n+1$ pairwise incompatible elements in $\bigcup_{i \leq n} F_{i}$. Suppose we constructed $\sigma$ as desired for every $i \leq n$, let $A_{n+1}$ be ALICE's move in the inning $n+1$ and $B_{n} \subset \bigcup_{i \leq n} F_{i}$ be as in the induction hypothesis. We have two cases to consider:

- If there is an $s \in A_{n+1}$ such that $s \perp t$ for all $t \in B_{n}$, then set $\sigma\left(\left\langle A_{i}: i \leq n\right\rangle^{\urcorner} A_{n+1}\right)=$ $F_{n+1}$, for every $F_{n+1} \subset A_{n+1}$ with $s \in F_{n+1}$ and we have the wished result.

- Otherwise, there must be $t \in B_{n}$ such that there exist $s_{1}, s_{2} \in A_{n+1}$ with $s_{1} \perp s_{2}$ and $s_{1}, s_{2} \leq t$. In this case, set $\sigma\left(\left\langle A_{i}: i \leq n\right\rangle^{\frown} A_{n+1}\right)=F_{n+1}=\left\{s_{1}, s_{2}\right\}$ and $B_{n+1}=$ $\left(B_{n} \backslash\{t\}\right) \cup\left\{s_{1}, s_{2}\right\}$ gives us what we need.

Finally, note that $\bigcup_{n \in \omega} B_{n} \subset \bigcup_{n \in \omega} F_{n}$ has $p$ in its closure, as intended.

In fact, we have the following more general result:

Proposition 4.2.7 ([7]). For each $k \in \mathbb{N}$ there is a countable space $X_{k}$ with only one non-isolated point $p_{k}$ on which ALICE $\uparrow \mathrm{G}_{\mathrm{k}}\left(\Omega_{p_{k}}, \Omega_{p_{k}}\right)$ and $\mathrm{BOB} \uparrow \mathrm{G}_{\mathrm{k}+1}\left(\Omega_{p_{k}}, \Omega_{p_{k}}\right)$.

This is already in great discrepancy with what we have seen in Theorem 4.1.37. But we get yet another divergence as a corollary of Example 4.2.4:

Corollary 4.2.8. Property $\mathrm{S}_{1}\left(\Omega_{x}, \Omega_{x}\right)$ does not imply that ALICE $\nmid \mathrm{G}_{1}\left(\Omega_{x}, \Omega_{x}\right)$, in general. 
Proof. If we consider the space $X$ from Example 4.2.4, note that $\mathrm{S}_{2}\left(\Omega_{x}, \Omega_{x}\right)$ holds over it, which implies (in view of Proposition 3.2.5) that $\mathrm{S}_{1}\left(\Omega_{x}, \Omega_{x}\right)$ also holds. However, ALICE $\uparrow G_{1}\left(\Omega_{x}, \Omega_{x}\right)$ over $X$.

Now let us further study $\mathrm{G}_{1}\left(\Omega_{x}, \Omega_{x}\right)$. As usual, we start by looking at some dual candidate:

Definition 4.2.9. Given a space $X$ and $x \in X$ we call the neighborhood-point game at $x$ the following game. In each inning $n \in \omega$, ALICE chooses an open neighborhood $V_{n}$ of $x$ and BоB responds with $x_{n} \in V_{n}$. Then the winner is ALICE if $\left\{x_{n}: n \in \omega\right\} \in \Omega_{x}$ (otherwise, BoB wins).

We will see in Theorem 4.2.11 that the neighborhood-point game is, indeed, dual to $\mathrm{G}_{1}\left(\Omega_{x}, \Omega_{x}\right)$. The proof of this duality is similar to the proof of the point-open and $\mathrm{G}_{1}(\mathscr{O}, \mathscr{O})$ 's duality:

Lemma 4.2.10. Let $\sigma$ be a strategy for Вов in $\mathrm{G}_{1}\left(\Omega_{x}, \Omega_{x}\right)$. Then for every $s \in{ }^{<\omega} \Omega_{x}$ there is an open set $V_{s}$ with $x \in V_{s}$ such that for every $y \in V_{s} \backslash\{x\}$ there is an $A_{y} \in \Omega_{x}$ such that $\sigma\left(s^{\wedge} A_{y}\right)=y$.

Proof. Consider $B=\left\{y \in X: \sigma\left(s^{\wedge} A\right) \neq y\right.$ for all $\left.A \in \Omega_{x}\right\}$. Then $B \notin \Omega_{x}$, so there is an open set $V_{s}$ with $x \in V_{s}$ such that $V_{s} \cap B=\emptyset$. It follows from the definition of $B$ that $V_{s}$ has the desired property.

Theorem 4.2.11. The games $\mathrm{G}_{1}\left(\Omega_{x}, \Omega_{x}\right)$ and the neighborhood-point game are dual.

Proof. Suppose $\gamma$ is a winning strategy for ALICE in the neighborhood-point game. We construct $\sigma$ as the following strategy for Вов in $\mathrm{G}_{1}\left(\Omega_{x}, \Omega_{x}\right)$ :

- in the first inning, if ALICE chooses $A_{0}$, set $\sigma\left(\left\langle A_{0}\right\rangle\right)=x_{0} \in A_{0} \cap \gamma(\langle\rangle)$;

- In general (that is, in the inning $n \in \omega)$, set $\sigma\left(\left\langle A_{0}, \ldots, A_{n}\right\rangle\right)=x_{n} \in A_{n} \cap \gamma\left(\left\langle x_{0}, \ldots, x_{n-1}\right\rangle\right)$.

It follows from the fact that $\gamma$ is a winning strategy that $x \in \overline{\left\{x_{n}: n \in \omega\right\}}$, hence, $\sigma$ is a winning strategy.

Now, assume that $\gamma$ is a winning strategy for ALICE in $\mathrm{G}_{1}\left(\Omega_{x}, \Omega_{x}\right)$. Set $\sigma$ as a strategy for Bов in the neighborhood-point game as follows:

- if AliCE chooses $V_{0}$ in the first inning, let $\sigma\left(\left\langle V_{0}\right\rangle\right)=x_{0} \in V_{0} \cap \gamma(\langle\rangle)$;

- In general, set $\sigma\left(\left\langle V_{0}, \ldots, V_{n}\right\rangle\right)=x_{n} \in V_{n} \cap \gamma\left(\left\langle V_{0}, \ldots, V_{n-1}\right\rangle\right)$.

It follows from the fact that $\gamma$ is a winning strategy that $x \notin \overline{\left\{x_{n}: n \in \omega\right\}}$, hence, $\sigma$ is a winning strategy.

Now, suppose that $\sigma$ is a winning strategy for Вов in the neighborhood-point game. Note that if $s$ is a sequence of open neighborhoods of $x$, then $x \in \overline{\left\{\sigma\left(s^{\wedge} V\right): V \text { open neighborhood of } x\right\}}$ 
(in fact, this is true regardless of $\sigma$ being a winning strategy). Then let $\gamma$ be the following strategy for ALICE in $\mathrm{G}_{1}\left(\Omega_{x}, \Omega_{x}\right)$ :

- in the first inning, let

$$
\gamma(\langle\rangle)=\{\sigma(\langle V\rangle): V \text { open neighborhood of } x\}
$$

and, if Вов responds with $x_{0} \in \gamma(\langle\rangle)$, let $V_{0}$ be the open neighborhood of $x$ such that $\sigma\left(\left\langle V_{0}\right\rangle\right)=x_{0}$

- In general, set

$$
\gamma\left(\left\langle x_{0}, \ldots, x_{n}\right\rangle\right)=\left\{\sigma\left(\left\langle V_{0}, \ldots, V_{n}\right\rangle^{\urcorner} V\right): V \text { open neighborhood of } x\right\}
$$

with each $V_{k}$ being such that $x_{k}=\sigma\left(\left\langle V_{0}, \ldots, V_{k}\right\rangle\right)$.

Since $\gamma$ forces Bов to play in $\mathrm{G}_{1}\left(\Omega_{x}, \Omega_{x}\right)$ with a run $\sigma$ would play in the neighborhood-point game, it is indeed a winning strategy.

Finally, assume that $\sigma$ is a winning strategy for Bов in $\mathrm{G}_{1}\left(\Omega_{x}, \Omega_{x}\right)$. We define $\gamma$ as the following strategy for ALICE in the neighborhood-point game (without loss of generality, we will assume that ВОВ never chooses $x$ ):

- In the first inning, let $\gamma(\langle\rangle)=V_{\langle\rangle}$, with $V_{\langle\rangle}$being as in Lemma 4.2.10 for $s=\langle\rangle$. If B OB responds with $x_{0} \in V_{\langle\rangle}$, we let $A_{x_{0}}$ be as in Lemma 4.2.10;

- in the next inning, set $\gamma\left(\left\langle x_{0}\right\rangle\right)=V_{\left\langle A_{x_{0}}\right\rangle}$. If BOB responds with $x_{1} \in V_{\left\langle A_{x_{0}}\right\rangle}$, we let $A_{x_{1}}$ be as in Lemma 4.2.10;

- in the next inning, set $\gamma\left(\left\langle x_{0}, x_{1}\right\rangle\right)=V_{\left\langle A_{x_{0}}, A_{x_{1}}\right\rangle}$. If BoB responds with $x_{2} \in V_{\left\langle A_{x_{0}}, A_{x_{1}}\right\rangle}$, we let $A_{x_{2}}$ be as in Lemma 4.2.10;

- and so on.

Since $\gamma$ forces Вов to play in the neighborhood-point game with a run $\sigma$ would play in $\mathrm{G}_{1}\left(\Omega_{x}, \Omega_{x}\right)$, it is indeed a winning strategy.

We also get a result analogous to Theorem 4.1.4:

Theorem 4.2.12 ([Gruenhage 1976]). If $X$ is a separable regular space and $x \in X$ is such that Bов $\uparrow \mathrm{G}_{1}\left(\Omega_{x}, \Omega_{x}\right)$, then $X$ is first countable at $x$.

Proof. Let $D$ be a countable dense subset of $X$ and $\gamma$ a winning strategy or AliCE in the neighborhood-point game (whose existence is assured by Theorem 4.2.11). 
We now consider every run played with $\gamma$ such that Bов chooses points from $D$. We let $\mathscr{B}$ be the collection of open sets played by $\gamma$ in these specific runs. Because $D$ is countable, $\mathscr{B}$ is countable.

To see that this is a local base for $X$ at $p$ we use regularity: suppose, striving for a contradiction, that there is an open set $V$ such that $B \not \subset V$ for all $B \in \mathscr{B}$. By regularity, there is an open set $W$ such that $p \in W \subset \bar{W} \subset V$. Then $B \not \subset \bar{W}$ for all $B \in \mathscr{B}$, which is a contradiction. Indeed, as ALICE plays any $B \in \mathscr{B}$, B ов can pick $x \in B \backslash \bar{W}$ and BoB will win the run, since every single point he picks is in the complement of $\bar{W}$.

One may wonder whether changing condition " $\left\{x_{n}: n \in \omega\right\} \in \Omega_{x}$ " to a stronger version " $\left\langle x_{n}: n \in \omega\right\rangle$ converges to $x$ " makes a difference in terms of the players having (or not having) winning strategies. Indeed, this is a well known game presented by Gruenhage:

Definition 4.2.13. Given a space $X$ and $p \in X$ we call the neighborhood-point convergence game at $p$ the following game. In each inning $n \in \omega$, ALICE chooses an open neighborhood $V_{n}$ of $p$ and Вов responds with $x_{n} \in V_{n}$. Then the winner is Alice if $\left\langle x_{n}: n \in \omega\right\rangle$ converges to $x$ (otherwise, Вов wins).

Surprisingly, this modification makes no difference for ALICE (regarding her having a winning strategy or not):

Theorem 4.2.14 ([Gruenhage 1976]). ALICE has a winning strategy in the neighborhood-point game at $x \in X$ if, and, only if, ALICE has a winning strategy in the neighborhood-point convergence game at $x$.

Proof. This proof will follow the steps of Theorem 2.3.16's proof:

Let $\gamma$ be a winning strategy for ALICE in the neighborhood-point game at $x$. Then we define a strategy $\tilde{\gamma}$ for ALICE in the neighborhood-point convergence game at $x$ as follows.

- First, we let

$$
\tilde{\gamma}(\langle\rangle)=\gamma(\langle\rangle)
$$

- If BoB chooses $x_{0} \in \tilde{\gamma}(\langle\rangle)$, we let

$$
\tilde{\gamma}\left(\left\langle x_{0}\right\rangle\right)=\gamma(\langle\rangle) \cap \gamma\left(\left\langle x_{0}\right\rangle\right) ;
$$

- If B Ов chooses $x_{1} \in \tilde{\gamma}\left(\left\langle x_{0}\right\rangle\right)$, we let

$$
\tilde{\gamma}\left(\left\langle x_{0}, x_{1}\right\rangle\right)=\gamma(\langle\rangle) \cap \gamma\left(\left\langle x_{0}\right\rangle\right) \cap \gamma\left(\left\langle x_{1}\right\rangle\right) \cap \gamma\left(\left\langle x_{0}, x_{1}\right\rangle\right) ;
$$

- In general, if Bов chooses $x_{n} \in \tilde{\gamma}\left(\left\langle x_{i}: i<n\right\rangle\right)$ we let $S$ be the (finite) collection of subsequences of $\left\langle x_{i}: i \leq n\right\rangle$ and then

$$
\tilde{\gamma}\left(\left\langle x_{i}: i<n\right\rangle^{\frown} x_{n}\right)=\bigcap_{s \in S} \gamma(s) .
$$


Striving for a contradiction, suppose there is a possible sequence $\left\langle x_{n}: n \in \omega\right\rangle$ played by B B B against $\tilde{\gamma}$ such that there is an an open neighborhood $V$ of $p$ and an infinite $I \subset \omega$ with $x_{i} \notin V$ for every $i \in I$. Fix an increasing enumeration $I=\left\{i_{k}: k \in \omega\right\}$. Then because of the way we constructed $\tilde{\gamma}$, the sequence $\left\langle x_{i_{k}}: k \in \omega\right\rangle$ can be played against $\gamma$. But this contradicts the fact that $\gamma$ is a winning strategy in the neighborhood-point game at $p$.

The other implication is trivial.

For Вов, on the other hand, it might be easier to have a winning strategy in the neighborhood-point convergence game. The following result illustrates this:

Proposition 4.2.15 ([Gruenhage 2006]). There is a countable space X with only one nonisolated point $x$ such that $\mathrm{B}$ Ов has a winning strategy in the neighborhood-point convergence game at $x$, but $\mathrm{B}$ ОВ has no winning strategy in the neighborhood-point game at $x$.

Later on we will show that $\mathrm{G}_{1}\left(\Omega_{x}, \Omega_{x}\right)$ is related to productively countably tight spaces. Now, we move on to another closure-related game: recall that $\mathrm{D}$ denotes the family of all dense subsets of a given space $X$. Immediately, we get that:

Proposition 4.2.16. If ALICE has no winning strategy in $\mathrm{G}_{\mathrm{fin}}(\mathrm{D}, \mathrm{D})$ over $X$, then $X$ is separable.

Proof. Suppose $X$ is not separable. If AliCE plays with $X$ in every inning, then Bob has just no chance of winning against this strategy.

The concept of $\pi$-basis is also related to $G_{1}(D, D)$ :

Definition 4.2.17. Let $X$ be a space. Then $\mathscr{B}$ is a $\pi$-basis if for every nonempty open set $V \subset X$ there is a nonempty $B \in \mathscr{B}$ such that $B \subset V$.

Proposition 4.2.18. If a space $X$ has countable $\pi$-basis, then Вов has a winning strategy in $\mathrm{G}_{1}(\mathrm{D}, \mathrm{D})$ over $X$.

Proof. Let $\mathscr{B}=B_{n}: n \in \omega$ be a $\pi$-basis for a space $X$. Then, for each $D_{n}$ dense played by ALICE, Bов can pick $d_{n} \in D_{n} \cap B_{n}$ and it is, then, easy to see that $\left\{d_{n}: n \in \omega\right\}$ will be dense in $X$.

But also, following the steps we made with $\mathrm{G}_{1}\left(\Omega_{x}, \Omega_{x}\right)$ we can also obtain a dual game analogously:

Definition 4.2.19. Given a space $X$ we call the open-point game the following game. In each inning $n \in \omega$, ALICE chooses an open set $V_{n}$ and В ОВ responds with $x_{n} \in V_{n}$. Then the winner is AliCE if $\left\{x_{n}: n \in \omega\right\}$ is dense in $X$ and Вов otherwise.

Theorem 4.2.20. The games $\mathrm{G}_{1}(\mathrm{D}, \mathrm{D})$ and the open-point game are dual. 
Exercise 4.2.21. Given a space $X$ and $x \in X$, we say $A \in \Omega_{x}$ is nontrivial if $x \notin \overline{\{y\}}$ for every $y \in A$.

In this case, show that if $\mathrm{BoB} \uparrow \mathrm{G}_{1}\left(\Omega_{x}, \Omega_{x}\right)$, then for every nontrivial $A \in \Omega_{x}$ there is an infinite $B \subset A$ such that $C \in \Omega_{x}$ for every infinite $C \subset B$.

Hint: Use Theorems 4.2.11 and 4.2.14.

Exercise 4.2.22. Write the details of Theorem 4.2.20's proof. 
CHAPTER

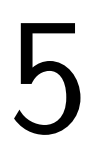

\section{SOME CONNECTIONS AND APPLICATIONS}

\subsection{The Banach-Mazur game and Baire spaces}

In the Banach-Mazur game's introduction it was mentioned that this game is connected to the Baire Category Theorem - we dedicate this section to establish and explore this connection. We start by recalling what a Baire space is:

Definition 5.1.1. We say a space $X$ is a Baire space if for every countable family $\mathscr{A}$ of dense open sets of $X, \cap \mathscr{A}$ is dense on $X$.

Note that if $X$ is a Baire space, then every open set $U \subset X$ is a Baire space. This will help us characterize such spaces:

Theorem 5.1.2 ([Oxtoby 1957]). A space $X$ is a Baire space if, and only if, $\operatorname{ALICE} \Varangle \operatorname{BM}(X)$.

Proof. Suppose $X$ is not a Baire space and let $\left\{A_{n}: n \in \omega\right\}$ be a countable family of dense open sets such that there is an open set $U_{0}$ with $\bigcap_{n \in \omega} A_{n} \cap U_{0}=\emptyset$. Then consider the following strategy $\gamma$ for ALICE in $\operatorname{BM}(X)$ :

- First, let $\gamma(\langle\rangle)=U_{0}$;

- Then if $V_{0}$ is BoB's first response, $V_{0} \cap A_{0} \subset V_{0}$ is not empty and therefore $\gamma\left(\left\langle V_{0}\right\rangle\right)=V_{0} \cap A_{0}$ is a valid reply for ALICE in the next inning;

- In the inning $n \in \mathbb{N}$, if B ОВ played with $\left\langle V_{i}: i<n\right\rangle$ thus far, set $\gamma\left(\left\langle V_{i}: i<n\right\rangle\right)=V_{n-1} \cap$ $A_{n-1}$ (again, this is a valid move because $A_{n-1}$ is open and dense).

Note that $\bigcap_{n \in \omega} V_{n} \subset \bigcap_{n \in \omega} A_{n} \cap U_{0}=\emptyset$. Hence, $\gamma$ is a winning strategy.

On the other hand, suppose $X$ is a Baire space and let $\gamma$ be a strategy for AlicE in $\operatorname{BM}(X)$. We define $S \subset \operatorname{dom}(\sigma)$ with the following recursion: 
(1) \langle\rangle$\in S$;

(2) If $s \in S$, then let $\mathscr{B}_{s}=\left\{V: s^{\urcorner} V \in S\right\}$ be a maximal family (obtained with the KuratowskiZorn Lemma or transfinite recursion) such that $\left\{\gamma\left(s^{\wedge} V\right): V \in \mathscr{B}_{s}\right\}$ is pairwise disjoint.

Now, let $B_{n}=\{s \in S:|s|=n\}$ and then, for each $n \in \omega$,

$$
A_{n}=\bigcup_{s \in B_{n}} \gamma(s)
$$

Claim 5.1.3. For every $n \in \omega, A_{n}$ is dense in $A_{0}=\gamma(\langle\rangle)$.

Proof. It suffices to show that $A_{n+1}$ is dense in $A_{n}$ for every $n \in \omega$, so fix $n \in \omega$ and let $A \subset A_{n}$ be a non-empty open set. Then $A \cap \gamma(s) \neq \emptyset$ for some $s \in B_{n}$. Note that, by maximality of $\mathscr{B}_{s}$, $\bigcup_{V \in \mathscr{B}_{s}} \gamma\left(s^{\urcorner} V\right)$ is dense in $\gamma(s)$, so there must be a $V \in \mathscr{B}_{s}$ such that $A \cap \gamma\left(s^{\wedge} V\right) \neq \emptyset$. Since $s^{\wedge} V \in B_{n+1}, A \cap A_{n+1} \neq \emptyset$.

Since $X$ is a Baire space, so is $A_{0}$, then let $x \in \bigcap_{n \in \omega} A_{n}$. Because $x \in A_{0}, x \in \gamma(\langle\rangle)$. Also, since $x \in A_{1}$ and $\left\{\gamma(\langle V\rangle): V \in \mathscr{B}_{\langle\rangle}\right\}$is pairwise disjoint, there must be a unique $V_{0} \in \mathscr{B}_{\langle\rangle}$such that $x \in \gamma\left(\left\langle V_{0}\right\rangle\right)$. Again, since $x \in A_{1}$ and $\left\{\gamma\left(\left\langle V_{0}, V\right\rangle\right): V \in \mathscr{B}_{\left\langle V_{0}\right\rangle}\right\}$ is pairwise disjoint, there must be a unique $V_{1} \in \mathscr{B}_{\left\langle V_{0}\right\rangle}$ such that $x \in \gamma\left(\left\langle V_{0}, V_{1}\right\rangle\right)$. By proceeding in this manner we find a sequence $\left\langle V_{n}: n \in \omega\right\rangle$ of open sets such that $\left\langle V_{i}: i \leq k\right\rangle \in S \subset \operatorname{dom}(\gamma)$ and $x \in \gamma\left(\left\langle V_{i}: i \leq k\right\rangle\right)$ for every $k \in \omega$, which means that $\gamma$ is not a winning strategy.

Now let us examine the characterization given by Theorem 5.1.2 a bit further. Immediately, we get as corollaries:

Corollary 5.1.4. If $X$ is a nonempty space such that $\mathrm{BoB} \uparrow \mathrm{BM}(X)$, then $X$ is a Baire space.

Corollary 5.1.5. If $X$ is a complete metric space, then $X$ is Baire.

Proof. Recall that in Example 2.5.2 it was shown that $\operatorname{BoB} \uparrow \operatorname{BM}(X)$.

Corollary 5.1.6. If $X$ is the space of irrational numbers, than $X$ is Baire.

Proof. Recall that in Example 2.5.3 it was shown that Вов $\uparrow \mathrm{BM}(X)$.

Corollary 5.1.7. $\mathbb{R}_{l}$ is Baire.

Proof. Recall that in Example 2.5.7 it was shown that $\mathrm{BoB} \uparrow \mathrm{BM}\left(\mathbb{R}_{l}\right)$.

Corollary 5.1.8. If $K$ is a compact Hausdorff space, then $K$ is Baire.

Proof. Recall that in Example 2.5.9 it was shown that $\mathrm{B}$ ов $\uparrow \mathrm{BM}(X)$. 
We have already seen in Proposition 2.5.13 that the Banach-Mazur game is undetermined, hence, the inverse implication of Corollary 5.1.4 does not hold. As a matter of fact, B oB having a winning strategy in the Banach-Mazur game is connected to a property that is strictly stronger than that of Baire spaces:

Definition 5.1.9. We say a Baire space $X$ is productively Baire if, for every Baire space $Y$, $X \times Y$ is also a Baire space.

Theorem 5.1.10. Let $X$ be a space. If $\mathrm{B}$ ов has a winning strategy in $\mathrm{BM}(X)$ then $X$ is productively Baire.

Proof. Let $\sigma$ be a winning strategy for Вов in $\operatorname{BM}(X)$ and suppose that there is a space $Y$ such that $X \times Y$ is not Baire. We will show that $Y$ must not be Baire.

By Theorem 5.1.2, ALICE has a winning strategy $\gamma$ in $\operatorname{BM}(X \times Y)$. Note that we may assume that $\gamma$ plays only with boxes (basic open sets). Now we can construct a winning strategy $\tilde{\gamma}$ for ALICE in $\operatorname{BM}(Y)$ as follows:

- In the first inning, set $\tilde{\gamma}(\langle\rangle)=B_{0}$, with $B_{0}$ being such that $\gamma(\langle\rangle)=A_{0} \times B_{0}$;

- When BoB responds with $V_{0}$, set $\tilde{\gamma}\left(\left\langle V_{0}\right\rangle\right)=B_{1}$, with $B_{1}$ being such that $\gamma\left(\left\langle\sigma\left(\left\langle A_{0}\right\rangle\right) \times V_{0}\right\rangle\right)=$ $A_{1} \times B_{1}$

- If BoB then chooses $V_{1}$, set $\tilde{\gamma}\left(\left\langle V_{0}, V_{1}\right\rangle\right)=B_{2}$, with $B_{2}$ being such that $\gamma\left(\left\langle\sigma\left(\left\langle A_{0}\right\rangle\right) \times V_{0}\right\rangle, \sigma\left(\left\langle A_{1}\right\rangle\right) \times\right.$ $\left.V_{1}\right)=A_{2} \times B_{2}$

- and so on.

Since $\gamma$ is a winning strategy for ALICE in $\operatorname{BM}(X \times Y)$,

$$
\emptyset=\bigcap_{n \in \omega} V_{n} \times \sigma\left(\left\langle B_{0}, \ldots, B_{n}\right\rangle\right)=\bigcap_{n \in \omega} V_{n} \times \bigcap_{n \in \omega} \sigma\left(\left\langle B_{0}, \ldots, B_{n}\right\rangle\right) .
$$

But, since $\sigma$ for Bов in $\operatorname{BM}(X)$,

$$
\bigcap_{n \in \omega} \sigma\left(\left\langle B_{0}, \ldots, B_{n}\right\rangle\right) \neq \emptyset
$$

so $\bigcap_{n \in \omega} V_{n}=\emptyset$ and, therefore, $\tilde{\gamma}$, is a winning strategy.

Indeed, it was shown in [Cohen 1976] that there is even a Baire metric space $X$ whose power $X^{2}$ is not Baire - which provides us yet another example of space on which the BanachMazur game is undetermined. Finally, Theorem 5.1.10 allows us to find some examples of productively Baire spaces:

Corollary 5.1.11. If $X$ is a complete metric space, then $X$ is productively Baire.

Corollary 5.1.12. $\mathbb{R}_{l}$ is productively Baire.

Corollary 5.1.13. If $K$ is a compact Hausdorff space, then $K$ is productively Baire. 


\subsection{The Rothberger game and Measure Theory}

In this section we examine how measure behaves on Rothberger spaces and, finally, give a definitive answer to question 2.3.13. We start with the concept of strong measure zero.

\subsubsection{Strong measure zero}

Definition 5.2.1. A metric space $\langle X, d\rangle$ has strong measure zero if for every sequence of positive numbers $\left\langle\varepsilon_{n}: n \in \omega\right\rangle$ there is a sequence $\left\langle I_{n}: n \in \omega\right\rangle$ of subsets of $X$ such that $X=$ $\bigcup_{n \in \omega} I_{n}$ and, for each $n \in \omega, \operatorname{diam}\left(I_{n}\right)<\varepsilon_{n}$.

Example 5.2.2. Clearly, every countable metric space has strong measure zero.

Example 5.2.3. Every open interval $I \subset \mathbb{R}$ does not have strong measure zero. Indeed, consider $a, b \in \mathbb{R}$ with $a<b$ and let $M=b-a$ (which is the Lebesgue measure of $I=] a, b[$ ). Then, for each $n \in \omega$, set $\varepsilon_{n}=\frac{M}{2^{n+1}}$. Note that for every sequence $\left\langle I_{n}: n \in \omega\right\rangle$ of subsets of $I$ such that, for each $n \in \omega, \operatorname{diam}\left(I_{n}\right)<\varepsilon_{n}$, the Lebesgue measure of $\bigcup_{n \in \omega} I_{n}$ is at most $M / 2$, which is strictly smaller then $M$. Hence, $\bigcup_{n \in \omega} I_{n} \neq I$.

In view of Examples 5.2.2 and 5.2.3 one may wonder whether there is an uncountable subset of the real line with strong measure zero. Borel's conjecture deals with this question:

Conjecture 5.2.4 (Borel's Conjecture). Every strong measure zero subset of the real line is countable.

As it turns out, Conjecture 5.2.4 is independent of ZFC! And this conjecture will allow us to answer Question 2.3.13. But first, let us show some preliminary results:

Theorem 5.2.5. If $\langle X, d\rangle$ is a metric space with strong measure zero, then $X$ is zero-dimensional.

Proof. Fix $x \in X$ and $\varepsilon>0$. Let $f: X \rightarrow \mathbb{R}^{\geq 0}$ be such that $f(y)=d(x, y)$.

CLAIM 5.2.6. $f[X]$ has strong measure zero.

Proof. Let $\left\langle\varepsilon_{n}: n \in \omega\right\rangle$ be a sequence of positive numbers and let $\left\langle I_{n}: n \in \omega\right\rangle$ be a sequence of subsets of $X$ such that $X=\bigcup_{n \in \omega} I_{n}$ and $\operatorname{diam}\left(I_{n}\right)<\varepsilon_{n}$ for each $n \in \omega$. It is clear that $f[X]=$ $\bigcup_{n \in \omega} f\left[I_{n}\right]$, so the result follows from the fact that

$$
\left|f(y)-f\left(y^{\prime}\right)\right|=\left|d(x, y)-d\left(x, y^{\prime}\right)\right| \leq d\left(y, y^{\prime}\right)
$$

which implies that $\operatorname{diam}\left(f\left[I_{n}\right]\right)<\varepsilon_{n}$.

Now, since $f[X]$ has strong measure zero, it follows from Example 5.2.3 that there is a $\delta \in] 0, \varepsilon[$ such that $f(y) \neq \delta$ for all $y \in X$. We conclude the proof by observing that

$$
f^{-1}\left(\left[0, \delta[)=f^{-1}([0, \delta]) \subset B_{\varepsilon}(x) .\right.\right.
$$


Theorem 5.2.7. If $\langle X, d\rangle$ is a metric space with strong measure zero, then $X$ is separable.

Proof. Since $X$ has strong measure zero, for each $k \in \mathbb{N}$ there is a sequence $\left\langle B_{1 / k}\left(x_{n}^{k}\right): n \in \mathbb{N}\right\rangle$ such that $X=\bigcup_{n \in \mathbb{N}} B_{1 / k}\left(x_{n}^{k}\right)$. We claim that $\left\{x_{n}^{k}: n, k \in \mathbb{N}\right\}$ is a dense subset of $X$. Indeed, let $B_{\varepsilon}(x)$ be an arbitrary open ball in $X$. Then there is a $k_{0} \in \mathbb{N}$ such that $1 / k_{0}<\varepsilon / 2$. On the other hand, since $X=\bigcup_{n \in \mathbb{N}} B_{1 / k_{0}}\left(x_{n}^{k_{0}}\right)$, there is an $n_{0} \in \mathbb{N}$ such that $x \in B_{1 / k_{0}}\left(x_{n_{0}}^{k_{0}}\right)$. It follows that $x_{n_{0}}^{k_{0}} \in B_{\mathcal{E}}(x)$, which concludes the proof.

Now we can finally present the connection between the strong measure zero property and $\mathrm{S}_{1}(\mathscr{O}, \mathscr{O})$ :

Theorem 5.2.8 ([Miller and Fremlin 1988]). Let X be a metrizable space. Then $X$ is Rothberger if, and only if, $\langle X, d\rangle$ has strong measure zero for every metric $d$ which generates $X$ 's topology.

Proof. Suppose $X$ is Rothberger, let $d$ be a metric which generates $X$ 's topology and $\left\langle\varepsilon_{n}: n \in \omega\right\rangle$ be a sequence of positive numbers. For each $n \in \omega$, let

$$
\mathscr{U}_{n}=\left\{B_{\varepsilon_{n} / 2}(x): x \in X\right\} .
$$

Then, since $X$ is Rothberger, there is an $x_{n} \in X$ for each $n \in \omega$ such that $X=\bigcup_{n \in \omega} B_{\varepsilon_{n} / 2}\left(x_{n}\right)$, which concludes the proof of the first implication.

Now, suppose $\langle X, d\rangle$ has strong measure zero for every metric $d$ which generates $X$ 's topology. Note that, by Theorems 5.2.5 and 5.2.7, $X$ is zero-dimensional and separable. Let $\left\langle\mathscr{U}_{n}: n \in \omega\right\rangle$ be a sequence of open covers and fix a metric $d$ which generates $X$ 's topology. Since $X$ is zero-dimensional, we may assume every element of $\mathscr{U}_{n}$ is clopen for all $n \in \omega$. In this case, since $X$ is separable, we may assume $\mathscr{U}_{n}=\left\{U_{n}^{k}: k \in \omega\right\}$ is a disjoint collection of clopen sets such that $\operatorname{diam}_{d}\left(U_{n}^{k}\right)<1 / n+1$ for all $k \in \omega$. By taking common refinements we may assume, at last, that $\mathscr{U}_{n+1}$ refines $\mathscr{U}_{n}$.

Note that, with all of these assumptions, $\mathscr{B}=\bigcup_{n \in \omega} \mathscr{U}_{n}$ is a basis for $X$. Then let $d^{\prime}: X \times$ $X \rightarrow \mathbb{R}^{\geq 0}$ be such that $d^{\prime}(x, y)=\frac{1}{n+1}$ with $n$ being the minimal natural number such that there is a $k \in \omega$ with $x \in U_{n}^{k}$ and $y \notin U_{n}^{k}$.

Claim 5.2.9. $d^{\prime}$ is a metric that generates $X$ 's topology.

Proof. It is clear that $d^{\prime}(x, y)=d^{\prime}(y, x)$ for all $x, y \in X$. Also, since $\operatorname{diam}_{d}\left(U_{n}^{k}\right)<\frac{1}{n+1}$ for all $k, n \in \omega$, it is clear that $d^{\prime}(x, y)=0$ if, and only if, $x=y$. It remains to prove the triangle inequality, so let $x, y, z \in X$. Let $n_{0}$ be such that $d^{\prime}(x, y)=\frac{1}{n_{0}+1}$ and let $U_{n_{0}}^{x}$ and $U_{n_{0}}^{y}$ be the disjoint clopen sets from $\mathscr{U}_{n_{0}}$ that contains $x$ and $y$, respectively. We have two possible cases here:

- if $z \notin U_{n_{0}}^{x}$ nor $z \notin U_{n_{0}}^{y}$, then $d^{\prime}(x, z), d^{\prime}(z, y)>d^{\prime}(x, y)$, which implies the triangle inequality for this case. 
- otherwise, if $z \in U_{n_{0}}^{x}$ (or $z \in U_{n_{0}}^{y}$ ), then $d^{\prime}(z, y) \geq d^{\prime}(x, y)$ (or $d^{\prime}(x, z) \geq d^{\prime}(x, y)$ ), which again implies the triangle inequality.

It follows that $d^{\prime}$ is a metric.

Now, given $x \in X$ and $\varepsilon>0$, let $B_{\varepsilon}^{d^{\prime}}(x)$ denote the open ball centered in $x$ with radius $\varepsilon$ with respect to $d^{\prime}$. Then note that, for each $n \in \omega, B_{\frac{1}{n+1}}^{d^{\prime}}(x)=U_{n}^{k}$, with $k \in \omega$ being such that $x \in U_{n}^{k}$, which shows that $d^{\prime}$ generates $X$ 's topology.

In this case, let $\operatorname{diam}_{d^{\prime}}: X \rightarrow \mathbb{R}^{\geq 0} \cup\{\infty\}$ be such that $\operatorname{diam}_{d^{\prime}}(A)$ denotes the diameter of $A$ with respect to $d^{\prime}$. Note that $\left\langle\frac{1}{n+2}: n \in \omega\right\rangle$ is a sequence of positive numbers, so there is a sequence $\left\langle I_{n}: n \in \omega\right\rangle$ of subsets of $X$ such that $X=\bigcup_{n \in \omega} I_{n}$ and $\operatorname{diam}_{d^{\prime}}\left(I_{n}\right)<\frac{1}{n+2}$ for all $n \in \omega$. Note that, because $\operatorname{diam}_{d^{\prime}}\left(I_{n}\right)<\frac{1}{n+2}, I_{n} \subset U_{n}^{k_{n}}$ for some $k_{n} \in \omega$. Then it follows that $X=\bigcup_{n \in \omega} U_{n}^{k_{n}}$, which concludes the proof.

Note that Theorem 5.2.8 gives us, in particular:

Corollary 5.2.10. If $X$ is a metrizable Rothberger space, then $X$ has strong measure zero.

Hence:

Corollary 5.2.11. If Borel's conjecture holds, then every metrizable Rothberger space is countable.

So we conclude, in view of Corollaries 4.1.35 and 5.2.11, that the answer to Question 2.3.13 is independent of ZFC (since, as already remarked, this question is translated to Question 3.3.10).

\subsubsection{Purely atomic measures}

Now, let us explore how regular, $\sigma$-finite Borel measures operate in regular Rothberger spaces. First, consider the following concept:

Definition 5.2.12. Given a measure $\mu$ on a $\sigma$-algebra $\mathscr{A}$, we say $E \in \mathscr{A}$ is an atom if for every $E^{\prime} \subset E$ such that $E^{\prime} \in \mathscr{A}$, either $\mu\left(E^{\prime}\right)=\mu(E)$, or else $\mu\left(E^{\prime}\right)=0$. We then say $\mu$ is a purely atomic measure if every $E \in \mathscr{A}$ such that $\mu(E)>0$ contains an atom.

Also, recall that a Borel measure $\mu$ is a regular measure if for every Borel set $B \subset X$

$$
\mu(B)=\inf _{B \subset U \in \tau} \mu(U) .
$$

Our objective is to show that every regular $\sigma$-finite Borel measure over a regular Rothberger space is purely atomic. In order to do that, we must first extend some measure notions to a broader class of functions: 
If $\mathscr{M}$ is a family of subsets of a space $X$ we say a function $\mu: \mathscr{M} \rightarrow \mathbb{R}^{\geq 0} \cup\{\infty\}$ is:

- finite, if $\mu(M)<\infty$ for all $M \in \mathscr{M}$;

- $\sigma$-finite if there is a countable set $\left\{M_{n}: n \in \omega\right\} \subset \mathscr{M}$ such that $\mu\left(M_{n}\right)<\infty$ for every $n \in \omega$ and $X=\bigcup_{n \in \omega} M_{n}$;

- countably additive if $\mu\left(\bigcup_{n \in \omega} M_{n}\right)=\sum_{n \in \omega} \mu\left(M_{n}\right)$ whenever $\left\{M_{n}: n \in \omega\right\} \subset \mathscr{M}$ is pairwise disjoint and $\bigcup_{n \in \omega} M_{n} \in \mathscr{M}$.

In what follows, given a space $X$, we will denote by $\mathscr{C}_{X}$ and $\mathscr{B}_{X}$ the family of all clopen sets of $X$ and the family of all Borel sets of $X$, respectively.

Lemma 5.2.13 ([Matveev 2010]). Let $X$ be a regular Rothberger space and let $\mu: \mathscr{C}_{X} \rightarrow$ $\mathbb{R}^{\geq 0} \cup\{\infty\}$ be a finite, countably additive function. Then there is a countable $M_{0} \subset X$ and $a$ function $m: M_{0} \rightarrow \mathbb{R}^{\geq 0}$ such that, for every $U \in \mathscr{C}_{X}$,

$$
\mu(U)=\sum_{x \in U \cap M_{0}} m(x) .
$$

Proof. For each $x \in X$, let

$$
m(x)=\inf \left\{\mu(U): U \in \mathscr{C}_{X} \text { and } x \in U\right\}
$$

And then, for each $\varepsilon \geq 0$, set $M_{\varepsilon}=\{x \in X: m(x)>\varepsilon\}$. Note that, for every $\varepsilon>0, M_{\varepsilon}$ is finite. Indeed, suppose it is infinite for some $\varepsilon>0$. Then, for every given $n \in \mathbb{N}$, there are distinct $x_{1}, \ldots x_{n} \in M_{\varepsilon}$ and sets $U_{1}, \ldots U_{n} \in \mathscr{C}_{X}$ such that $x_{k} \in U_{k}$ for each $0<k \leq n$ and $\left\{U_{k}: 0<k \leq n\right\}$ is pairwise disjoint (recall that, by Theorem 3.3.13, $X$ is zero dimensional). Let $U_{0}=X \backslash\left(\bigcup_{1 \leq k \leq n} U_{k}\right)$. Then, for each $1 \leq k \leq n, \mu\left(U_{k}\right) \geq m\left(x_{k}\right)>\varepsilon$, so $\mu(X)=\sum_{k \leq n} \mu\left(U_{k}\right) \geq$ $\sum_{k \leq n} m\left(x_{k}\right) \geq n \varepsilon$, which implies that $\mu(X)=\infty$, a contradiction.

Note that $M_{0}=\bigcup_{n \in \mathbb{N}} M_{\frac{1}{n}}$, so $M_{0}$ is countable. Let $\mu_{m}: \mathscr{C}_{X} \rightarrow \mathbb{R}^{\geq 0}$, be such that $\mu_{m}(U)=$ $\sum_{x \in U \cap M_{0}} m(x)$. Then $\mu_{m}$ is finite, monotonic and $\sigma$-additive. Moreover:

Claim 5.2.14. For every $U \in \mathscr{C}_{X}, \mu(U) \geq \mu_{m}(U)$.

Proof. Let $U \in \mathscr{C}_{X}$ and write $\left\{x_{n}: n \in \omega\right\}=U \cap M_{0}$. Then, for each $k \in \omega, \mu(U) \geq \sum_{n=0}^{n=k} m\left(x_{n}\right)$, so it follows that $\mu(U) \geq \sum_{n=0}^{\infty} m\left(x_{n}\right)=\mu_{m}(U)$.

Let $\mu_{r}: \mathscr{C}_{X} \rightarrow \mathbb{R}^{\geq 0}$ be such that $\mu_{r}(U)=\mu(U)-\mu_{m}(U)$.

CLAIM 5.2.15. $\mu_{r} \equiv 0$, so $\mu \equiv \mu_{m}$.

Proof. Fix $x \in X$ and $\varepsilon>0$. Then, from the definition of $m(x)$, there is an $U \in \mathscr{C}_{X}$ such that $x \in U$ and $\mu(U)-m(x)<\frac{1}{2}$, so it follows from the previous claim that

$$
\mu(U)-\mu_{m}(U)=\mu(U)-m(x)-\left(\mu_{m}(U)-m(x)\right)<\varepsilon-\left(\mu_{m}(U)-m(x)\right)<\varepsilon .
$$


In this case, given $\varepsilon>0, \mathscr{U}_{\varepsilon}=\left\{U \in \mathscr{C}_{X}: \mu_{r}(U)<\varepsilon\right\}$ is an open cover of $X$. Suppose (striving for a contradiction) that $\mu_{r}(X)>0$. Then let, for each $n \in \omega, \varepsilon_{n}=\frac{1}{2^{n+1}} \mu_{r}(X)$. Since $X$ is Rothberger, there is a $U_{n} \in \mathscr{U}_{\varepsilon_{n}}$ for each $n \in \omega$ such that $X=\bigcup_{n \in \omega} U_{n}$. Then $\mu_{r}(X) \leq$ $\sum_{n \in \omega} \mu_{r}\left(U_{n}\right)=\frac{1}{2} \mu_{r}(X)$, a contradiction.

And the proof is complete.

Theorem 5.2.16 ([Matveev 2010]). Let X be a regular Rothberger space and let $\mu$ be a regular, $\sigma$-finite Borel measure on $X$. Then there is a countable $M_{0} \subset X$ and a function $m: M_{0} \rightarrow \mathbb{R} \geq 0$ such that, for every $B \in \mathscr{B}_{X}$,

$$
\mu(B)=\sum_{x \in M_{0} \cap B} m(x)
$$

Proof. Let $\mathscr{U}=\left\{B_{n}: n \in \omega\right\} \subset \mathscr{B}_{X}$ be such that $X=\bigcup_{n \in \omega} B_{n}$ and $\mu\left(B_{n}\right)<\infty$ for every $n \in \omega$ (we may assume that $\mathscr{U}$ is pairwise disjoint). Then, for each $n \in \omega$, set $\mu_{n}: \mathscr{B}_{X} \rightarrow \mathbb{R}^{\geq 0} \cup\{\infty\}$ be such that $\mu_{n}(B)=\mu\left(B \cap B_{n}\right)$. Note that $\mu_{n}$ restricted to $\mathscr{C}_{X}$ satisfies the conditions of Lemma 5.2.13, so let $M_{0}^{n} \subset X$ and $m_{n}: M_{0}^{n} \rightarrow \mathbb{R}^{\geq 0}$ be such that $\mu_{n}(U)=\sum_{x \in U \cap M_{0}^{n}} m_{n}(x)$ for all $U \in \mathscr{C}_{X}$. CLAiM 5.2.17. For all $n \in \omega$ and $B \in \mathscr{B}_{X}, \mu_{n}(B)=\sum_{x \in B \cap M_{0}^{n}} m_{n}(x)$.

Proof. Indeed, by regularity, $\mu_{n}(\{x\})=m_{n}(x)$ for all $x \in X$, so if we put $\mu_{n}^{m}(B)=\sum_{x \in B \cap M_{0}^{n}} m_{n}(x)$ and $\mu_{n}^{r}=\mu_{n}-\mu_{n}^{a}$, then $\mu_{n}^{r}$ is a non-negative monotonic function. By Lemma 5.2.13, on the other hand, $\mu_{n}^{r}(X)=0$, so it follows that $\mu_{n}^{r}(B)=0$ for all $B \in \mathscr{B}_{X}$ and, therefore, $\mu_{n}(B)=$ $\sum_{x \in B \cap M_{0}^{n}} m_{n}(x)$.

Let $M_{0}=\bigcup_{n \in \mathbb{N}} M_{0}^{n}$ and $m: M_{0} \rightarrow \mathbb{R}^{\geq 0}$ be such that $m(x)=m_{n}(x)$ with $n \in \omega$ being such that $x \in M_{0}^{n}$. The result then follows from the fact that, for all $B \in \mathscr{B}_{X}$

$$
\mu(B)=\sum_{n \in \omega} \mu\left(B \cap B_{n}\right)=\sum_{n \in \omega} \mu_{n}(B)=\sum_{n \in \omega} \sum_{x \in B \cap M_{0}^{n}} m_{n}(x)=\sum_{x \in B \cap M_{0}} m(x)
$$

Corollary 5.2.18. Let $X$ be a regular Rothberger space and let $\mu$ be a regular, $\sigma$-finite Borel measure on $X$. Then $\mu$ is purely atomic.

Proof. Indeed, in view of Theorem 5.2.16, $\left\{\{x\}: x \in M_{0}\right\}$ is a collection of Borel sets that attests that $\mu$ is purely atomic.

Exercise 5.2.19. Show that the assumption that $\mu$ is finite in Lemma 5.2.13 may be replace by the assumption that $\mu$ is $\sigma$-finite, that is, show that if $X$ is a Hausdorff Rothberger space and $\mu: \mathscr{C}_{X} \rightarrow \mathbb{R}^{\geq 0} \cup\{\infty\}$ is a $\sigma$-finite countably additive function, then there is a countable $M_{0} \subset X$ and a function $m: M_{0} \rightarrow \mathbb{R}^{\geq 0}$ such that, for every $U \in \mathscr{C}_{X}$,

$$
\mu(U)=\sum_{x \in U \cap M_{0}} m(x) .
$$




\subsection{Productively Lindelöf spaces}

We will now see how productively Lindelöf spaces relate to some of the previously presented selection principles. As one would expect:

Definition 5.3.1. A space $X$ is productively Lindelöf if, for every Lindelöf space $Y, X \times Y$ is Lindelöf.

Obviously, a productively Lindelöf space is also Lindelöf. Compact spaces are known examples of productively Lindelöf spaces. The usual proof uses the following lemma:

Lemma 5.3.2 (Tube Lemma). Let $K$ be a compact space. Then for every given space $Y$ and open cover $\mathscr{U}$ for $K \times Y$ there is for each $y \in Y$ an open neighborhood $V_{y}$ of $y$ and a finite $\mathscr{F} y \subset \mathscr{U}$ such that $K \times V_{y} \subset \bigcup \mathscr{F}_{y}$.

Proof. Fix a space $Y$, an open cover $\mathscr{U}$ for $K \times Y$ and $y \in Y$. For each $x \in K$, let $U(x)$ be an open subset of $K$ and $V(x)$ be an open subset of $Y$ such that $\langle x, y\rangle \in U(x) \times V(x) \subset U$ for some $U \in \mathscr{U}$. Note that $\{U(x): x \in K\}$ is an open cover for $K$, so fix a finite $F \subset X$ such that $\{U(x): x \in F\}$ covers $K$. Set $V_{y}=\bigcap_{x \in F} V(x)$ and a finite $\mathscr{F} y \subset \mathscr{U}$ such that, for each $x \in F, U(x) \times V(x) \subset U$ for some $U \in \mathscr{F}_{y}$. Then $K \times V_{y} \subset \bigcup \mathscr{F}_{y}$ and the proof is complete.

Theorem 5.3.3. If $K$ is a compact space, then $K$ is productively Lindelöf.

Proof. Let $Y$ be a Lindelöf space and $\mathscr{U}$ be an open cover for $K \times Y$. For each $y \in Y$, let $V_{y}$ and $\mathscr{F}_{y}$ be as in Lemma 5.3.2. Since $\left\{V_{y}: y \in Y\right\}$ is an open cover for $Y$, which is Lindelöf, there is a countable $\left\{y_{n}: n \in \omega\right\} \subset Y$ such that $\bigcup_{n \in \omega} V_{y_{n}}=Y$. It follows that $\bigcup_{n \in \omega} \mathscr{F}_{y_{n}}$ is a countable subcover of $\mathscr{U}$, which concludes the proof.

As it turns out, we can actually generalize Lemma 5.3.2 in order to generalize Theorem 5.3.3 to Alster spaces:

Lemma 5.3.4. Given spaces $X, Y$, a compact $K \subset X$ and an open cover $\mathscr{U}$ for $X \times Y$ there is for each $y \in Y$ an open neighborhood $V_{y}$ of $y$, an open $U_{y} \subset X$ with $K \subset U_{y}$, and a finite $\mathscr{F}_{y} \subset \mathscr{U}$ such that $U_{y} \times V_{y} \subset \bigcup \mathscr{F}_{y}$.

Proof. Fix a space $Y$, an open cover $\mathscr{U}$ for $K \times Y$ and $y \in Y$. For each $x \in K$, let $U(x)$ be an open subset of $K$ and $V(x)$ be an open subset of $Y$ such that $\langle x, y\rangle \in U(x) \times V(x) \subset U$ for some $U \in \mathscr{U}$. Note that $\{U(x): x \in K\}$ is an open cover for $K$, so fix a finite $F \subset X$ such that $\{U(x): x \in F\}$ covers $K$. Set $V_{y}=\bigcap_{x \in F} V(x), U_{y}=\bigcup_{x \in F} U(x)$ and a finite $\mathscr{F}_{y} \subset \mathscr{U}$ such that, for each $x \in F$, $U(x) \times V(x) \subset U$ for some $U \in \mathscr{F}_{y}$. Then $U \times V_{y} \subset \bigcup \mathscr{F}_{y}$ and the proof is complete.

Theorem 5.3.5. If $X$ is an Alster space, then $X$ is productively Lindelöf. 
Proof. Let $Y$ be a Lindelöf space and $\mathscr{U}$ be an open cover for $X \times Y$. For each compact $K \subset X$ and $y \in Y$ let $V_{y}(K), U_{y}(K)$ and $\mathscr{F}_{y}(K)$ be as in Lemma 5.3.4. Since $\left\{V_{y}(K): y \in Y\right\}$ is an open cover for $Y$, which is Lindelöf, there is a countable $\left\{y_{k}: k \in \omega\right\} \subset Y$ such that $\bigcup_{k \in \omega} V_{y_{k}}(K)=Y$. Then $G(K)=\bigcap_{k \in \omega} \cup U_{y_{k}}(K)$ is a $G_{\delta}$ subset of $X$ containing $K$. Note that $\{G(K): K \subset X$ compact $\}$ is an Alster cover for $X$, so there is a countable collection $\left\{K_{n}: n \in \omega\right\}$ of compact subsets of $X$ such that $\bigcup_{n \in \omega} G\left(K_{n}\right)=X$. It follows that $\bigcup_{n \in \omega} \bigcup_{k \in \omega} \mathscr{F}_{y_{k}}\left(K_{n}\right)$ is a countable subcover of $\mathscr{U}$.

We should note that in [Alster 1988] it was also shown that if we assume $\mathrm{CH}$ and that $X$ has a basis of cardinality $\aleph_{1}$ (or less), then the converse of Theorem 5.3.5 actually holds. But Alster's selection principle is not the only one related to the property of being a productively Lindelöf space: $\mathrm{S}_{\text {fin }}(\mathscr{O}, \mathscr{O})$ also has a surprising connection. In order to see that, we recall that in [Michael 1971] it was shown that, under $\mathrm{CH}$, there is a Lindelöf space $X$ whose product with the space of irrational numbers is not Lindelöf and it was asked whether this would be true even without $\mathrm{CH}$ :

Question 5.3.6 (Michael's problem). Is there a Lindelöf space $X$ whose product with the space of irrational numbers is not Lindelöf?

A space that satisfies the conditions of Question 5.3.6 is called a Michael space. In [Alster 1990] it was shown that, assuming Martin's Axiom and the negation of $\mathrm{CH}$, there is a Michael space. But what does all of this have to do with Menger spaces? To clarify this out, we need to consider some new concepts:

Definition 5.3.7. Given sets $X$ and $Y$, we say a function $\phi: X \rightarrow \wp \Theta(Y)$ is a set-valued map and, given $A \subset X$, we write $\phi(A)=\bigcup_{x \in A} \phi(x)$.

Moreover, if $X$ and $Y$ are spaces, we say that $\phi$ is:

- compact-valued if $\phi(x)$ is compact for every $x \in X$;

- upper semicontinuos if for every open $V \subset Y$, the set $\phi_{\subset}^{-1}(V)=\{x \in X: \phi(x) \subset V\}$ is open in $X$.

Immediately, we have some simple results, which will be left as exercises (5.3.13).

Proposition 5.3.8. Let $X$ and $Y$ be spaces with $X$ being Lindelöf. If $\phi: X \rightarrow \mathcal{O}_{(Y)}$ is a compactvalued upper semicontinuos map such that $\phi(X)=Y$, then $Y$ is Lindelöf.

Proposition 5.3.9. Suppose $\phi_{0}: X_{0} \rightarrow{ }\left(Y_{0}\right)$ and $\phi_{1}: X_{1} \rightarrow \wp\left(Y_{1}\right)$ are compact-valued upper semicontinuos maps. Then the function $\phi_{0} \times \phi_{1}: X_{0} \times X_{1} \rightarrow \mathcal{S}\left(Y_{0} \times Y_{1}\right)$ that assigns $\left(x_{0}, x_{1}\right)$ to $\phi_{0}\left(x_{0}\right) \times \phi_{1}\left(x_{1}\right)$ is also a compact-valued upper semicontinuous map. 
In view of Theorem 1.2.19, the connection between Menger spaces and Question 5.3.6 rises from the following result:

Lemma 5.3.10 ([Zdomskyy 2005]). Property $\mathrm{S}_{\mathrm{fin}}(\mathscr{O}, \mathscr{O})$ holds on a Lindelöf space if, and only if, $\phi(X) \neq \mathbb{N}^{\omega}$ for every compact-valued upper semicontinuous map $\phi: X \rightarrow \circlearrowleft\left(\mathbb{N}^{\omega}\right)$.

Theorem 5.3.11 ([Repovš and Zdomskyy 2012]). If there is a Michael space, then every productively Lindelöf space is Menger.

Proof. Striving for a contradiction, suppose that $X$ is a productively Lindelöf space such that $\mathrm{S}_{\mathrm{fin}}(\mathscr{O}, \mathscr{O})$ does not hold over $X$ and let $Y$ be a Michael space (we will show that $X \times Y$ is not Lindelöf).

Using Lemma 5.3.10, let $\phi: X \rightarrow \circlearrowleft\left(\mathbb{N}^{\omega}\right)$ be a compact-valued upper semicontinuous map such that $\phi(X)=\mathbb{N}^{\omega}$. Then, using Proposition 5.3.9 with $\phi_{0}=\phi$ and $\phi_{1}: Y \rightarrow \mathcal{O}_{(Y) \text { such }}$ that $\phi_{1}(y)=\{y\}, \mathbb{N}^{\omega} \times Y$ is the image of a compact-valued upper semicontinuous map. But, by the definition of a Michael space and Theorem1.2.19, $\mathbb{N}^{\omega} \times Y$ is not Lindelöf. Hence, $X \times Y$ is not Lindelöf, a contradiction.

Corollary 5.3.12. Assuming CH (or Martin's Axiom), every productively Lindelöf space is Menger.

Hence, we conclude that, under CH (or Martin's Axiom), the property of being productively Lindelöf lies between those of being Alster and Menger.

Exercise 5.3.13. Write the details of the proof of Propositions 5.3.8 and 5.3.8.

\subsection{Sieve completeness}

We now present a connection between compact-like spaces and another game that appears when we consider compactifications. This game goes as follows:

Definition 5.4.1. We call the sieve game the following game:

- At first, AliCE chooses an open cover $\mathscr{U}_{0}$ for $X$, then Bob chooses $U_{0} \in \mathscr{U}_{0}$;

- in each inning $n \in \mathbb{N}$ ALICE chooses an open cover $\mathscr{U}_{n}$ for $U_{n-1}$ and BoB chooses $U_{n} \in \mathscr{U}_{n}$.

We say AliCE wins if, for every filter base $\mathscr{F}$ (see Definition 1.1.6) such that for each $n \in \omega$ there is an $F_{n} \in \mathscr{F}$ with $F_{n} \subset U_{n}, \mathscr{F}$ clusters in $X$.

A winning strategy for ALICE in the sieve game is also called a complete sieve and a space with a complete sieve is said to be sieve-complete. 
The connection between compact-like and sieve-complete spaces in the context of compactification is then spelled out by the following results.

Theorem 5.4.2 ([Telgársky 1983]). If $X$ is a $T_{3 \frac{1}{2}}$ sieve-complete space and $Y$ is a compactification of $X$, then $Y \backslash X$ is compact-like.

Proof. Let $\gamma$ be a winning strategy in the sieve game over $X$. For each $U$ open subset of $X$, fix an open set $U^{\prime}$ in $Y$ such that $U=U^{\prime} \cap X$. Then, for each of $\gamma$ 's moves $\gamma\left(\left\langle V_{0}, \ldots V_{n}\right\rangle\right)$, let

$$
\gamma^{\prime}\left(\left\langle V_{0}, \ldots V_{n}\right\rangle\right)=\left\{U^{\prime}: U \in \gamma\left(\left\langle V_{0}, \ldots V_{n}\right\rangle\right)\right\}
$$

Now, let $K_{0}=Y \backslash \bigcup \gamma^{\prime}(\langle\rangle)$ be ALICE's first move in the compact-open game on $Y \backslash X$ (which is clearly a compact subset of $Y \backslash X$ ). Then, if В ов chooses $U_{0}^{*}=U_{0}^{\prime} \cap Y \backslash X \supset K_{0}$ (with $U_{0}^{\prime}$ open in $Y$ ), note that $\gamma^{\prime}(\langle\rangle)$ is an open cover for $Y \backslash U_{0}$ (which is compact in $Y$ ), so let $\mathscr{F}_{0}$ be its finite subcover. Set $S_{0}=\left\langle\left\langle V_{0}\right\rangle \in \operatorname{dom}(\gamma): V_{0} \in \mathscr{F}_{0}\right\rangle$ and then

$$
K_{1}=Y \backslash\left(U_{0}^{\prime} \cup \bigcup_{t \in S_{0}} \bigcup \gamma^{\prime}(t)\right),
$$

which is, again, clearly a compact subset of $Y \backslash X$. Once BоB responds with an open $U_{1}^{*}=$ $U_{1}^{\prime} \cap Y \backslash X$ (with $U_{0}^{\prime}$ open in $Y$ ), note again that $Y \backslash\left(U_{0} \cup U_{1}\right)$ is a compact subset of $Y$ covered by $\left\{U: U \in \gamma^{\prime}(\langle V\rangle), V \in \mathscr{F}_{0}\right\}$, so let $\mathscr{F}_{1}$ be its finite subcover. Set $S_{1}=\left\{\left\langle V_{0}, V_{1}\right\rangle \in \operatorname{dom}(\gamma): V_{0}^{\prime} \in \mathscr{F}_{0}, V_{1}^{\prime} \in \mathscr{F}_{1}\right\}$ and then

$$
K_{2}=Y \backslash\left(U_{0}^{\prime} \cup U_{1}^{\prime} \cup \bigcup_{t \in S_{1}} \bigcup \gamma^{\prime}(t)\right),
$$

and so on. Now let $y \in Y \backslash \bigcup_{n \in \omega} U_{n}^{\prime}$ (we will show that $y \in X$, which concludes the proof). Then

$$
y \in \bigcap_{n \in \omega} \bigcup_{V_{n}^{\prime} \in \mathscr{F}_{n}} V_{n}^{\prime} .
$$

Claim 5.4.3. There is a sequence $\left\langle V_{n}: n \in \omega\right\rangle$ such that, for each $k \in \omega,\left\langle V_{i} \leq k\right\rangle \in \operatorname{dom}(\gamma)$, $V_{k} \in \mathscr{F}_{k}$ and

$$
y \in \bigcap_{n \in \omega} V_{n}^{\prime}
$$

Proof. Let $T=\bigcup_{n \in \omega} S_{n}$ with the order $\leq$ defined as follows: $t \leq s$ if, and only if, $t \subset s$. Since each $\mathscr{F}_{n}$ is finite, $\langle T, \leq\rangle$ is a finitely branching tree. And, by $5.1, T$ is infinite, so our result follows from König's Lemma (see 1.1.5).

Since each $V$ is dense in $V^{\prime}$,

$$
y \in \bigcap_{n \in \omega} \bar{V}_{n} .
$$

Now, let $\mathscr{B}_{y}$ be a local basis at $y$ and set

$$
\mathscr{F}=\left\{B \cap V_{n}: B \in \mathscr{B}_{y}, n \in \omega\right\} .
$$


Clearly, $\mathscr{F}$ is a filter base and for every $n \in \omega$ there is an $F_{n} \in \mathscr{F}$ such that $F_{n} \subset V_{n}$, so $\mathscr{F}$ clusters at some $x \in X$. But, because $Y$ is Hausdorff, $\mathscr{F}$ can only cluster at $y$. It follows that $y=x \in X$, and the proof is complete.

Theorem 5.4.4 ([Telgársky 1983]). If $X$ is a $T_{3 \frac{1}{2}}$ compact-like space and $Y$ is a compact Hausdorff space containing $X$, then $Y \backslash X$ is sieve-complete.

Proof. Let $\gamma$ be a winning strategy for ALICE in the compact-open game over $X$. We define a winning strategy for ALICE in the sieve game over $Y \backslash X$ as follows:

For starters, let $\mathscr{U}_{0}^{\prime}$ be the collection $Y$ 's open sets $U_{0}^{\prime}$ such that $\overline{U_{0}^{\prime}} \cap \gamma(\langle\rangle)=\emptyset$. Then, since $Y$ is regular, $\mathscr{U}_{0}^{\prime}$ covers $Y \backslash X$, so let $\mathscr{U}_{0}=\left\{U_{0}^{\prime} \cap(Y \backslash X): U_{0}^{\prime} \in \mathscr{U}_{0}^{\prime}\right\}$ be AliCE's initial move in the sieve game over $Y \backslash X$.

Once Bов chooses $U_{0}=U_{0}^{\prime} \cap(Y \backslash X) \in \mathscr{U}_{0}$, let $V_{0}=X \backslash \overline{U_{0}^{\prime}}$ be BoB's response for $\gamma(\langle\rangle)$ in the compact-open game over $X$. Note that the collection $\mathscr{U}_{1}^{\prime}$ of open sets $U_{1}^{\prime}$ of $Y$ such that $\overline{U_{1}^{\prime}} \cap \gamma\left(\left\langle V_{1}\right\rangle\right)$ again covers $Y \backslash X$, so we let ALICE's response for $U_{0} \in \mathscr{U}_{0}$ in the sieve game over $Y \backslash X$ be

$$
\mathscr{U}_{1}=\left\{U_{1}^{\prime} \cap U_{0}: U_{1}^{\prime} \in \mathscr{U}_{1}^{\prime}\right\}
$$

and so on. To show that this is indeed a winning strategy, let $\mathscr{F} \subset \mathscr{⿰}(Y \backslash X)$ be a filter base such that for each $n \in \omega$ there is an $F_{n} \in \mathscr{F}$ with $F_{n} \subset U_{n}$. Note that, since $Y$ is compact, there is a $y \in Y$ such that $\mathscr{F}$ clusters at $y$ (see Theorem 1.2.17), that is, $y \in \bigcap_{B \in \mathscr{F}} \bar{B}$. If that is the case, then $y \in \bigcap_{n \in \omega} \overline{U_{n}}$, which implies that $y \notin \bigcup_{n \in \omega}\left(X \backslash \overline{U_{n}}\right)$. But, since $\gamma$ is a winning strategy in the compact-open game, $X=\bigcup_{n \in \omega}\left(X \backslash \overline{U_{n}}\right)$, so it follows that $y \in Y \backslash X$, and the proof is complete.

Corollary 5.4.5 ([Telgársky 1983]). A $T_{3 \frac{1}{2}}$ space $X$ is sieve-complete if, and only if, $\beta X \backslash X$ is compact-like.

\section{5 - D-spaces}

One of the applications of covering games is related to the so called $D$-spaces (a property that appears to have been first introduced in exchanged letters between E.K. van Douwen and E. Michael in the mid-1970s). To clarify these applications, we begin with the definition of such spaces:

Definition 5.5.1. An open neighborhood assignment (also known as o.n.a.) in a space $X$ is a function from $X$ into its topology which assigns to each $x \in X$ one of its open neighborhoods.

We then say $X$ is a $D$-space if for every o.n.a. $\left\{V_{x}: x \in X\right\}$ there is a closed discrete subspace $D \subset X$ such that $\left\{V_{x}: x \in D\right\}$ covers $X$. 
The most trivial examples of $D$-spaces are discrete or compact $T_{1}$ spaces. But what other spaces are $D$ ? One may wonder, for instance, if it is possible to use the Lindelöf property to show that a space is $D$. Indeed, this problem was proposed by van Douwen and remains open thus far. But if we suppose something stronger then the Lindelöf property, we may find an answer and, as a bonus, find a whole class of examples of $D$-spaces:

Theorem 5.5.2 ([Aurichi 2010]). If $X$ is a $T_{1}$ space such that $\mathrm{S}_{\text {fin }}(\mathscr{O}, \mathscr{O})$ holds on $X$, then $X$ is a D-space.

Proof. Let $\mathscr{V}=\left\{V_{x}: x \in X\right\}$ be an o.n.a. We define a strategy $\gamma$ for AlICE in $\mathrm{G}_{\text {fin }}(\mathscr{O}, \mathscr{O})$ as follows:

- First, let $\gamma(\langle\rangle)=\mathscr{V}$;

- if B OB responds with a finite $\mathscr{F}_{0} \subset \gamma(\langle\rangle)$, let $F_{0} \subset X$ be a finite set such that $\left\{V_{x}: x \in F_{0}\right\}=$ $\mathscr{F}_{0}$. Then set

$$
\gamma\left(\left\langle\mathscr{F}_{0}\right\rangle\right)=\left\{V_{x}: x \in X \backslash \bigcup \mathscr{F}_{0}\right\} \cup\left\{\bigcup \mathscr{F}_{0}\right\}
$$

- if Вов responds with a finite $\mathscr{F}_{1} \subset \gamma(\langle\rangle)$, let $F_{1} \subset X$ be a finite set such that $\left\{V_{x}: x \in F_{1}\right\}=$ $\mathscr{F}_{1}$ (we may assume that BоB's choice does not contain $\bigcup \mathscr{F}_{0}$, since he already covered this portion of the space in the previous inning). Then set

$$
\gamma\left(\left\langle\mathscr{F}_{0}, \mathscr{F}_{1}\right\rangle\right)=\left\{V_{x}: x \in X \backslash \bigcup_{i \leq 1} \bigcup \mathscr{F}_{i}\right\} \cup\left\{\bigcup \mathscr{F}_{i}: i \leq 1\right\} ;
$$

- if B ОB responds with a finite $\mathscr{F}_{2} \subset \gamma(\langle\rangle)$, let $F_{2} \subset X$ be a finite set such that $\left\{V_{x}: x \in F_{2}\right\}=$ $\mathscr{F}_{2}$ (again, we may assume that BoB's choice does not contain $\bigcup \mathscr{F}_{0}$ or $\bigcup \mathscr{F}_{1}$, since he already covered both portions of the space in the previous innings). Then set

$$
\gamma\left(\left\langle\mathscr{F}_{0}, \mathscr{F}_{1}, \mathscr{F}_{2}\right\rangle\right)=\left\{V_{x}: x \in X \backslash \bigcup_{i \leq 2} \bigcup \mathscr{F}_{i}\right\} \cup\left\{\bigcup \mathscr{F}_{i}: i \leq 2\right\} ;
$$

- and so on.

By Theorem 4.1.12, ALICE $\nmid \mathrm{G}_{\text {fin }}(\mathscr{O}, \mathscr{O})$, so there is a run $\left\langle\mathscr{F}_{n}: n \in \omega\right\rangle$ compatible with $\gamma$ such that $\bigcup_{n \in \omega} \mathscr{F}_{n} \in \mathscr{O}$. We claim that $D=\bigcup_{n \in \omega} F_{n}$ is a closed discrete subset of $X$. Indeed, let $y \in X \backslash D$. Since $\bigcup_{n \in \omega} \mathscr{F}_{n} \in \mathscr{O}$, there is a $k \in \omega$ such that $y \in U_{1}=\bigcup_{j \leq k} \mathscr{F}_{j}$. Note that, by construction, $\bigcup_{j \leq k} \mathscr{F}_{j} \cap \bigcup_{n \geq k} F_{n}=\emptyset$. On the other hand, $\bigcup_{j \leq k} F_{n}$ is finite and $X$ is $T_{1}$, so there is an open neighborhood $U_{2}$ of $y$ such that $U_{2} \cap \bigcup_{j \leq k} F_{n}=\emptyset$. It follows that $U_{1} \cap U_{2}$ is an open neighborhood of $y$ that does not intersect $D$ and, therefore, $D$ is closed. Now, fix $x \in D$. Then $x \in F_{k}$ for some $k \in \omega$. Note that $x \in U_{1}=\bigcup_{j \leq k} \mathscr{F}_{j}$ and again, by construction, $\bigcup_{j \leq k} \mathscr{F}_{j} \cap \bigcup_{n \geq k} F_{n}=\emptyset$. On the other hand, $\bigcup_{j \leq k} F_{n}$ is finite and $X$ is $T_{1}$, so there is an open neighborhood $U_{2}$ of $x$ such that $U_{2} \cap \bigcup_{j \leq k} F_{n}=\emptyset$. It follows that $U=U_{1} \cap U_{2}$ is an open 
neighborhood of $x$ such that $U \cap D=\{x\}$ and, therefore, $D$ is also discrete, which concludes the proof.

Corollary 5.5.3. If $X$ is a $T_{1}$ Rothberger space, then $X$ is a $D$-space.

Corollary 5.5.4. If $X$ is a $T_{1}$ space such that $\mathrm{B}$ ОВ has no winning strategy in the compact-open game, then $X$ is a D-space.

Proof. If Вов has no winning strategy in the compact-open game over $X$, by Theorem 4.1.17, ALICE has no winning strategy in $\mathrm{G}_{\text {fin }}(\mathscr{O}, \mathscr{O})$, which implies that $\mathrm{S}_{\text {fin }}(\mathscr{O}, \mathscr{O})$ holds and, by Theorem 5.5.2, $X$ is a $D$-space.

Corollary 5.5.5. If $X$ is a $T_{1}$ space such that $\mathrm{B}$ ОВ has no winning strategy in the point-open game, then $X$ is a $D$-space.

It is also unknown whether even finite unions of $D$-spaces are also $D$. In the particular case of the examples we have just presented, on the other hand, we do know:

Corollary 5.5.6. If $X=\bigcup_{n \in \omega} X_{n}$ with each $X_{n}$ being a $T_{1}$ space on which $\mathrm{S}_{\mathrm{fin}}(\mathscr{O}, \mathscr{O})$ holds, then $X$ is a D-space.

Proof. This is clear if we consider Proposition 3.3.3 and Theorem 5.5.2.

And we can go even further if we look at a new game:

Definition 5.5.7. We call the DC-open game the following game on a space $X$. In each inning $n \in \omega:$

- ALICE chooses a discrete collection $\mathscr{K}_{n}$ of compact sets;

- BOB chooses an open set $V_{n} \supset \bigcup \mathscr{K}_{n}$.

We say that ALICE wins if $X=\bigcup_{n \in \omega} V_{n}$ and B OB wins otherwise.

We then say a space is DC-like if ALICE has a winning strategy in the DC-open game.

Again, the most trivial example of DC-like spaces are those spaces which are union of a discrete family of compact sets. But also:

Example 5.5.8. Note that if ALICE has a winning strategy in the point-open game (or in the compact-open game) over a space $X$, then $X$ is DC-like.

Now, how does DC-like spaces relate to $D$-spaces? Well:

Lemma 5.5.9 ([Peng 1996]). If $X$ is a $T_{1} D C$-like space, then $X$ is a D-space. 
Proof. Let $\gamma$ be a winning strategy in the DC-open game over $X$ and let $\left\{V_{x}: x \in X\right\}$ be an o.n.a.. We may assume that, for every $s \in \operatorname{dom}(\gamma), K \cap \bigcup_{k \in \operatorname{dom}(s)} s(k)=\emptyset$ for all $K \in \gamma(s)$. For each $K \in \gamma(\langle\rangle)$, note that $\left\{V_{x}: x \in K\right\}$ is an open cover for $K$, so let $F_{K}$ be the finite subset of $X$ such that $\left\{V_{x}: x \in F_{K}\right\}$ covers $K$. Then set

$$
D_{0}=\bigcup_{K \in \gamma(\langle\rangle)} F_{K}
$$

and note that $D_{0}$ is a closed (because $X$ is $T_{1}$ ) discrete subset o $X$ and $U_{0}=\bigcup_{x \in D_{0}} V_{x} \supset \bigcup \gamma(\langle\rangle)$.

Now, note again that, for each $K \in \gamma\left(\left\langle U_{0}\right\rangle\right)$, note that $\left\{V_{x}: x \in K\right\}$ is an open cover for $K$, so let $F_{K}$ be the finite subset of $X$ such that $\left\{V_{x}: x \in F_{K}\right\}$ covers $K$. Then set

$$
D_{1}=\bigcup_{K \in \gamma\left(\left\langle U_{0}\right\rangle\right)} F_{K}
$$

and note that $D_{1}$ is also a closed (again, because $X$ is $T_{1}$ ) discrete subset and $U_{1}=\bigcup_{x \in D_{1}} V_{x} \supset$ $\bigcup \gamma\left(\left\langle U_{0}\right\rangle\right)$.

By continuing with this process we define for each $n \in \omega$ a $D_{n} \subset X$ such that $U_{n}=$ $\bigcup_{x \in D_{1}} V_{x} \supset \gamma\left(\left\langle U_{0}, \ldots U_{n-1}\right\rangle\right)$ and $D_{0} \cup \cdots \cup D_{n}$ is discrete. Then we set $D=\bigcup_{n \in \omega} D_{n}$. Since $\gamma$ is a winning strategy, note that $\left\{V_{x}: x \in D\right\}$ covers $X$. Moreover, being a discrete union of discrete sets, $D$ is also discrete. In order to complete the proof it remains to show, therefore, that $D$ is closed. Indeed, let $y \in X \backslash D$. Note that there is an $n \in \omega$ such that $y \in V_{x}$ or some $x \in D_{n}$ and, by our initial assumption $V_{x} \cap \bigcup_{k \geq n} D_{k}=\emptyset$, so $y \notin \overline{\bigcup_{k \geq n} D_{k}}$. But, since $X$ is $T_{1}$, $y \notin \bigcup_{k \leq n} D_{k}=\overline{\bigcup_{k \leq n} D_{k}}$, our result follows from the identity

$$
\bar{D}=\left(\overline{\bigcup_{k \leq n} D_{k}}\right) \cup\left(\overline{\bigcup_{k \geq n} D_{k}}\right) .
$$

Theorem 5.5.10 ([Peng 2008]). If $X$ is a $T_{1}$ space such that $X=\bigcup_{k \leq n} X_{k}$ for some $n \in \omega$ with each $X_{k}$ being $D C$-like, then $X$ is a D-space.

Proof. By induction it suffices to show that $X=X_{1} \cup X_{2}$ with both $X_{1}$ and $X_{2}$ being DC-like spaces is a $D$-space.

Let $\left\{V_{x}: x \in X\right\}$ be an o.n.a. and $\gamma_{1}, \gamma_{2}$ be winnings strategies for ALICE in the DC-open games over $X_{1}$ and $X_{2}$, respectively. We may assume that, for every $i \in\{1,2\}$ and for every $s \in \operatorname{dom}\left(\gamma_{i}\right), K \cap \bigcup_{k \in \operatorname{dom}(s)} s(k)=\emptyset$ for all $K \in \gamma_{i}(s)$. Let

$$
A_{0}^{1}=\{x \in X: \gamma(\langle\rangle) \text { is not locally finite at } x\} .
$$

Then, by Proposition 1.2.15, $A_{0}^{1} \subset X_{2}$ is closed in $X$. Since $X_{2}$ is DC-like, it follows from Lemma 5.5.9 that we can find a $D_{0}^{1 *} \subset A_{0}^{1}$ closed and discrete in $X$ such that $\left\{V_{x}: x \in D_{0}^{1 *}\right\}$ covers $A_{0}^{1}$. 
In this case,

$$
\mathscr{K}_{0}^{1}=\left\{K^{*}=K \backslash \bigcup_{x \in D_{0}^{1 *}} V_{x}: K \in \gamma_{1}(\langle\rangle)\right\}
$$

is a discrete family of compact subsets of $X$, so that for each $K^{*}$ we may find a finite $F_{K^{*}} \subset K^{*}$ such that $\left\{V_{x}: x \in F_{K^{*}}\right\}$ covers $K^{*}$. Note that $\bigcup_{K^{*} \in \mathscr{K}_{0}^{1}} F_{K^{*}}$ is closed (because $X$ is $T_{1}$ ) and discrete in $X$, so if we let $D_{0}^{1}=D_{0}^{1 *} \cup_{K^{*} \in \mathscr{K}_{0}^{1}} F_{K^{*}}$, then $D_{0}^{1}$ is also closed and discrete and

$$
\bigcup \gamma_{1}(\langle\rangle) \subset \bigcup_{x \in D_{0}^{1}} V_{x}
$$

Repeat the exact same process in $X_{2}$ with $\gamma_{2}$ to find a closed discrete $D_{0}^{2}$ such that

$$
\bigcup \gamma_{2}(\langle\rangle) \subset \bigcup_{x \in D_{0}^{2}} V_{x}
$$

and then let $D_{0}=D_{0}^{1} \cup D_{0}^{2}$, so that $D_{0}$ is a closed discrete subset of $X$ and $U_{0}=\subset \bigcup_{x \in D_{0}} V_{x}$ covers both $\gamma_{1}(\langle\rangle)$ and $\gamma_{2}(\langle\rangle)$.

Now, let

$$
A_{1}^{1}=\left\{x \in X: \gamma\left(\left\langle U_{0} \cap X_{1}\right\rangle\right) \text { is not locally finite at } x\right\}
$$

Then, by Proposition 1.2.15, $A_{1}^{1} \subset X_{2}$ is closed in $X$. Since $X_{2}$ is DC-like, it follows from Lemma 5.5.9 that we can find a $D_{1}^{1 *} \subset A_{1}^{1}$ closed and discrete in $X$ such that $\left\{V_{x}: x \in D_{1}^{1 *}\right\}$ covers $A_{1}^{1}$. In this case,

$$
\mathscr{K}_{1}^{1}=\left\{K^{*}=K \backslash \bigcup_{x \in D_{1}^{1 *}} V_{x}: K \in \gamma_{1}\left(\left\langle U_{0} \cap X_{1}\right\rangle\right)\right\}
$$

is a discrete family of compact subsets of $X$, so that for each $K^{*}$ we may find a finite $F_{K^{*}} \subset K^{*}$ such that $\left\{V_{x}: x \in F_{K^{*}}\right\}$ covers $K^{*}$. Note that $\bigcup_{K^{*} \in \mathscr{K}_{1}^{1}} F_{K^{*}}$ is closed (again, because $X$ is $T_{1}$ ) and discrete in $X$, so if we let $D_{0}^{1}=D_{0}^{1 *} \cup_{K^{*} \in \mathscr{K}_{1}^{1}} F_{K^{*}}$, then $D_{1}^{1}$ is also closed and discrete and

$$
\bigcup \gamma_{1}\left(\left\langle U_{0} \cap X_{1}\right\rangle\right) \subset \bigcup_{x \in D_{1}^{1}} V_{x} .
$$

Again, repeat the exact same process in $X_{2}$ with $\gamma_{2}$ to find a closed discrete $D_{1}^{2}$ such that

$$
\bigcup \gamma_{2}\left(\left\langle U_{0} \cap X_{2}\right\rangle\right) \subset \bigcup_{x \in D_{1}^{2}} V_{x}
$$

and then let $D_{1}=D_{1}^{1} \cup D_{1}^{2}$, so that $D_{1}$ is a closed discrete subset of $X$ and $U_{1}=\subset \bigcup_{x \in D_{1}} V_{x}$ covers both $\gamma_{1}\left(\left\langle U_{0} \cap X_{1}\right\rangle\right)$ and $\gamma_{2}\left(\left\langle U_{0} \cap X_{2}\right\rangle\right)$.

By repeating this process we find for each $n \in \omega$ a closed discrete $D_{n} \subset X$ such that $U_{n}=\bigcup_{x \in D_{n}}$ covers both $\gamma_{1}\left(\left\langle U_{0} \cap X_{1}, \ldots, U_{n-1} \cap X_{1}\right\rangle\right)$ and $\gamma_{2}\left(\left\langle U_{0} \cap X_{2}, \ldots, U_{n-1} \cap X_{2}\right\rangle\right)$. 
Claim 5.5.11. $D=\bigcup_{n \in \omega} D_{n}$ is a closed discrete subset of $X$ such that $X=\bigcup_{x \in D} V_{x}$.

Proof. Because $\gamma_{1}$ and $\gamma_{2}$ are winning strategies over $X_{1}$ and $X_{2}$, respectively, and $X=X_{1} \cup X_{2}$, it is clear that $X=\bigcup_{x \in D} V_{x}$.

Now, let $x \in D$. Then $x \in D_{k}$ for some $k \in \omega$. Note that, by construction, $U_{k}$ is an open set containing $x$ such that $U_{k} \cap \bigcup_{n \geq k} D_{n}=\emptyset$, so it follows from the fact that $\bigcup_{n \leq k} D_{n}$ is discrete that $D$ is also discrete.

On the other hand, let $x \in X \backslash D$. Then $x \in U_{k}$ for some $k \in \omega$. Again, note that, by construction, $U_{k} \cap \bigcup_{n \geq k} D_{n}=\emptyset$, so it follows from the fact that $\bigcup_{n \leq k} D_{n}$ is closed that $D$ is also closed.

Then the proof is complete.

Exercise 5.5.12. Given a space $X$, let $G(D C, X)$ denote the following game : at first, ALICE chooses a discrete family $\mathscr{K}_{0}$ of compact subsets of $X$ and Вов responds with a closed $E_{0} \subset$ $X \backslash \bigcup \mathscr{K}_{0}$. In the inning $n \in \mathbb{N}$ ALICE chooses a discrete family $\mathscr{K}_{n}$ of compact subsets of $X$ and ВОВ responds with a closed $E_{n} \subset E_{n-1} \backslash \bigcup K_{n}$. ALICE wins if $\bigcap_{n \in \omega} E_{n}=\emptyset$ and BoB wins otherwise.

Show that $G(D C, X)$ is equivalent to the DC-open game.

Curiosity: the property of being DC-like was first introduced in [Telgársky 1983] with the game $G(D C, X)$.

\subsection{Tightness games and countable tightness}

Strong fan tightness and $\mathrm{G}_{1}\left(\Omega_{x}, \Omega_{x}\right)$ have an interesting relationship with countable tightness. This property goes as follows:

Definition 5.6.1. We say a space $X$ is countably tight at a point $x \in X$ if for every $A \in \Omega_{x}$ there is a countable $B \subset A$ such that $B \in \Omega_{x}$. If $X$ is countably tight at every point $x \in X$, then we simply say that $X$ is countably tight.

Obviously, every space with countable strong fan tightness is countably tight - so one may wonder whether the converse also holds. As the following example shows us, the answer is no:

Example 5.6.2 ([Arhangel'skii 1972]). Consider the following space:

$$
S_{\mathfrak{c}}=\bigcup_{\alpha<\mathfrak{c}}\left\{z_{n}^{\alpha}: n \in \omega\right\} \cup\{0\},
$$

with all the $z_{n}^{\alpha}$ 's distinct and isolated in $S_{\mathfrak{c}}$ and, if $f \in \omega^{\mathfrak{c}}$, then

$$
V_{f}=\{0\} \cup \bigcup_{\alpha<\mathfrak{c}}\left\{z_{n}^{\alpha}: n \geq f(\alpha)\right\}
$$


is a basic neighborhood of 0 .

Proposition 5.6.3. $S_{\mathfrak{c}}$ is countably tight.

Proof. Suppose $A \in \Omega_{0}$. We claim that there is an $\alpha_{0} \in \mathfrak{c}$ such that $A \cap\left\{z_{n}^{\alpha_{0}}: n \in \omega\right\}$ is infinite. Indeed, if there is none, we let, for each $\alpha \in \mathfrak{c}$,

$$
f(\alpha)=\max \left\{n \in \omega: z_{n}^{\alpha} \in A\right\}+1,
$$

and then $V_{f} \cap A$ would be empty, a contradiction. Now we claim that $A \cap\left\{z_{n}^{\alpha_{0}}: n \in \omega\right\} \in \Omega_{0}$. Indeed, given $f: \mathfrak{c} \rightarrow \omega$, there must be an $n \in \omega$ such that $n \geq f\left(\alpha_{0}\right)$ and $z_{n}^{\alpha_{0}} \in A \cap\left\{z_{n}^{\alpha_{0}}: n \in \omega\right\}$ (because the latter is infinite). So $A \cap\left\{z_{n}^{\alpha_{0}}: n \in \omega\right\} \cap V_{f} \neq \emptyset$ and the proof is complete.

\section{However:}

Proposition 5.6.4. $S_{1}\left(\Omega_{0}, \Omega_{0}\right)$ does not hold on $S_{\mathfrak{c}}$.

Proof. For each $k \in \omega$, let

$$
A_{k}=\left\{z_{n}^{k}: n \in \omega\right\} \in \Omega_{0} .
$$

Then, if $z_{n_{k}}^{k} \in A_{k}$ is picked for each $k \in \omega$, we may let $f: \mathfrak{c} \rightarrow n$ be

$$
f(\alpha)=\left\{\begin{array}{l}
n_{k}+1, \text { if } \alpha=k \text { for some } k \in \omega \\
0, \text { otherwise }
\end{array}\right.
$$

so that $V_{f} \cap\left\{z_{n_{k}}^{k}: k \in \omega\right\}=\emptyset$.

But if we strengthen our assumption to countable tightness in some product, then the implication holds:

Theorem 5.6.5 ([Aurichi and Bella 2014]). Let $X$ be a space. If $X \times S_{\mathfrak{c}}$ is countably tight at $(x, 0)$, then $\mathrm{S}_{1}\left(\Omega_{x}, \Omega_{x}\right)$ holds.

Proof. Let $\left\langle A_{n}: n \in \omega\right\rangle$ be a sequence such that $A_{n} \in \Omega_{x}$ for all $n \in \omega$ (our goal is to choose for each $n \in \omega$ a point $a_{n} \in A_{n}$ in such a way that $\left\{a_{n}: n \in \omega\right\} \in \Omega_{x}$ ). Since $X$ has countable tightness, we may assume that each one of the $A_{n}$ 's is countable. If we put $Y=\{x\} \cup \bigcup_{n \in \omega} A_{n}$, without loss of generality, we then can fix a collection $\mathscr{V}=\left\{U_{\alpha}: \alpha<\mathfrak{c}\right\}$ as a local basis at $x$ in the subspace $Y$ (since $Y$ is countable). Now, fix an almost disjoint family $\mathscr{R}=\left\{R_{\alpha}: \alpha<\mathfrak{c}\right\}$ of infinite subsets of $\omega$. Next, we assume that for every $n \in R_{\alpha}$ we can pick a point $x_{n}^{\alpha} \in A_{n} \cap U_{\alpha}$ in such a way that $x_{n}^{\alpha} \neq x_{m}^{\alpha}$ whenever $n \neq m$ (because, otherwise, the principle would be trivially satisfied for $\left\langle A_{n}: n \in \omega\right\rangle$ ), and then let $E_{\alpha}=\left\{x_{n}^{\alpha}: n \in R_{\alpha}\right\}$.

Now let us take a look at $X \times S_{\mathfrak{c}}$. Consider

$$
A=\bigcup_{\alpha<\mathfrak{c}}\left\{\left\langle x_{n}^{\alpha}, z_{n}^{\alpha}\right\rangle: n \in R_{\alpha}\right\}
$$


CLAIM 5.6.6. $\langle x, 0\rangle \in \bar{A}$

Proof. Let $U$ and $V(f)$ be open neighborhoods of $x$ and 0 , respectively. There must be an $\alpha<\mathfrak{c}$ such that $U_{\alpha} \cap Y \subset U$, so that $E_{\alpha} \subset U$. If, then, we pick $n \in R_{\alpha}$ in such a way that $n \geq f(\alpha)$,

$$
\left\langle x_{n}^{\alpha}, z_{n}^{\alpha}\right\rangle \in(U \times V(f)) \cap A
$$

as, desired.

Since $X \times S_{\mathfrak{c}}$ is countably tight at $\langle x, 0\rangle$, there is a countable set $F \subset \mathfrak{c}$ such that if

$$
B=\bigcup_{\alpha \in F}\left\{\left\langle x_{n}^{\alpha}, z_{n}^{\alpha}\right\rangle: n \in R_{\alpha}\right\}
$$

then $x \in \bar{B}$.

Claim 5.6.7. For every $U$ open neighborhood of $x,\left|U \cap E_{\alpha}\right|=\aleph_{0}$ for some $\alpha \in F$.

Proof. Suppose not, that is, there is an open neighborhood $U$ of $x$ such that, for every $\alpha \in F$, $U \cap E_{\alpha}$ is finite. Then for each $\alpha \in F$ fix $n_{\alpha} \in \omega$ in such a way that if $n \in R_{\alpha} \backslash n_{\alpha}$, then $x_{n}^{\alpha} \notin U$. Define $f \in \omega^{\mathfrak{c}}$ as

$$
f(\alpha)=\left\{\begin{array}{l}
n_{\alpha}, \text { if } \alpha \in F, \\
0, \text { otherwise }
\end{array}\right.
$$

Now, if we consider the open neighborhood $U \times V(f)$ of $\langle x, 0\rangle$, we get an $\alpha \in F$ and a $n \in R_{\alpha}$ such that $\left\langle x_{n}^{\alpha}, z_{n}^{\alpha}\right\rangle \in U \times V(f)$. Since $x_{n}^{\alpha} \in U, n<n_{\alpha}$. On the other hand, since $z_{n}^{\alpha} \in V(f)$, $n \geq f(\alpha)=n_{\alpha}$, a contradiction.

Finally, after enumerating $F=\left\{\alpha_{n}: n \in \omega\right\}$, we define, for each $n \in \omega$ :

$$
\begin{aligned}
& S_{\alpha_{n}}=R_{\alpha_{n}} \backslash\left(\bigcup_{k<n} R_{\alpha_{k}}\right), \\
& D_{\alpha_{n}}=\left\{x_{n}^{\alpha_{n}}: n \in S_{\alpha_{n}}\right\},
\end{aligned}
$$

so that the $S_{\alpha_{n}}$ s are pairwise disjoint and $S_{\alpha_{n}}$ differs from $R_{\alpha_{n}}$ only in finitely many points. If $n \in \omega$ is such that $n \in S_{\alpha_{k}}$ for some (unique!) $k \in \omega$, let $a_{n}=x_{n}^{\alpha_{k}} \in A_{n}$ and pick $a_{n} \in A_{n}$ arbitrarily, otherwise. Now, if $U$ is an open neighborhood of $x$, by Claim 5.6.7, there is a $k \in \omega$ such that $\left|U \cap D_{\alpha_{k}}\right|=\aleph_{0}$, so there is an $n \in S_{\alpha_{k}}$ such that $x_{n}^{\alpha_{k}} \in U$. Since $a_{n}=x_{n}^{\alpha_{k}}$, we have just proven that $p \in \overline{\left\{a_{n}: n \in \omega\right\}}$ as it was required.

As a bonus, in view of Example 5.6.2 and Theorem 5.6.5, we conclude that countable tightness does not behave well under products. More precisely:

Corollary 5.6.8. $S_{\mathfrak{c}} \times S_{\mathfrak{c}}$ is not countably tight at $\langle 0,0\rangle$. 
This motivates the definition of productively countably tight spaces:

Definition 5.6.9. We say a space $X$ is productively countably tight at a point $x \in X$ if for every $Y$ countably tight at a point $y, X \times Y$ is countably tight at $\langle x, y\rangle$. If $X$ is productively countably tight at every point $x \in X$, then we simply say that $X$ is productively countably tight.

And then we immediately get from Theorem 5.6.5:

Corollary 5.6.10. If $X$ is productively countably tight at $x \in X$, then $\mathrm{S}_{1}\left(\Omega_{x}, \Omega_{x}\right)$ holds.

Corollary 5.6.11. If $X$ is productively countably tight, then $\mathrm{S}_{1}\left(\Omega_{x}, \Omega_{x}\right)$ holds for every $x \in X$.

Corollary 5.6.10 gives us a necessary condition for productive countable tightness - but what about a sufficient condition? As it turns out, we can find one by exploring the tightness game $\mathrm{G}_{1}\left(\Omega_{x}, \Omega_{x}\right)$.

Theorem 5.6.12 ([Gruenhage 1976]). If $X$ is a space such that $\mathrm{BoB} \uparrow G_{1}\left(\Omega_{p}, \Omega_{p}\right)$ for some $p \in X$, then $X$ is productively countably tight at $p$.

Proof. Let $Y$ be a countably tight space at $q$, fix $A \in \Omega_{\langle p, q\rangle}$ and, using Theorem 4.2.11, let $\gamma$ be a winning strategy for ALICE in the neighborhood-point game at $p$. We will associate for each $s \in{ }^{<\omega} \omega$ an open $U_{s} \subset X$ and, for each $n \in \omega,\left\langle x_{s^{\urcorner} n}, y_{s^{\urcorner} n}\right\rangle \in A$ such that,

(1) $q \in \overline{\left\{y_{s^{\curvearrowright} n}: n \in \omega\right\}}$ for every $s \in{ }^{<\omega} \omega$;

(2) for every nonempty $s \in{ }^{<\omega} \omega,\left\langle x_{s \uparrow k}: 0<k \leq \operatorname{dom}(s)\right\rangle \in \operatorname{dom}(\gamma)$.

Indeed, suppose $U_{s\lceil k}$ and $\left\langle x_{s\left\lceil k^{\wedge} n\right.}, y_{s\left\lceil k^{\wedge} n\right.}\right\rangle$ are defined as desired for every $k<\operatorname{dom}(s)$ and $n \in \omega$. Then, first, set $U_{s}=\gamma\left(\left\langle x_{s \mid k}: k<\operatorname{dom}(s)\right\rangle\right)$. Now, let $B=\left\{y \in Y:\langle y, x\rangle \in A\right.$ and $\left.x \in U_{s}\right\}$ and note that $q \in \bar{B}$, so, since $Y$ is countably tight at $q$, we may pick a countable $B^{\prime} \subset C$ so that, by letting $x_{s^{\urcorner} n}$ and $y_{s^{\urcorner} n}$ be such that $\left\{\left\langle x_{s^{\urcorner} n}, y_{s^{\urcorner} n}\right\rangle: n \in \omega\right\}=B^{\prime}$, the recursion is complete.

We claim that $\langle p, q\rangle \in \overline{\left\{\left\langle x_{s}, y_{s}\right\rangle: s \in{ }^{<\omega} \omega\right\}}$, which concludes the proof. Indeed, fix a basic open neighborhood $U \times V$ of $\langle p, q\rangle$. Using condition (1) we may (recursively) find an $R \in$ ${ }^{\omega} \omega$ such that $y_{R \nmid k} \in V$ for every $k \in \omega$. On the other hand, in view of condition (2), $\left\langle x_{R \nmid k}: k \in \mathbb{N}\right\rangle$ is a run compatible with $\gamma$, so $x_{R \mid n} \in U$ for some $n \in \omega$. It follows that $\left\langle x_{R \mid n}, y_{R \mid n}\right\rangle \in U \times V$, hence, $\langle p, q\rangle \in \overline{\left.\left\{x_{s}, y_{s}\right\rangle: s \in{ }^{<\omega} \omega\right\}}$.

\subsection{The space of real continuous functions}

Some covering games on completely regular spaces have a surprising connection with tightness games in a specific associated hyperspace. We explore this connection in this chapter and this will allow us to finally show a space on which $\mathrm{S}_{\mathrm{fin}}\left(\Omega_{x}, \Omega_{x}\right)$ holds, but $\mathrm{S}_{1}\left(\Omega_{x}, \Omega_{x}\right)$ does not. We begin with the definition of such hyperspaces: 
Definition 5.7.1. Given a space $X$, let $\boldsymbol{C}(\boldsymbol{X}) \subset \mathbb{R}^{X}$ be such that $f \in C(X)$ if, and only if, $f: X \rightarrow \mathbb{R}$ is continuous (considering $\mathbb{R}$ with the usual topology). We then define $\boldsymbol{C}_{\boldsymbol{p}}(\boldsymbol{X})$ as $C(X)$ with the subspace topology (considering $\mathbb{R}^{X}$ with the product topology).

Given $x \in X$, we denote the projection of $\mathbb{R}^{X}$ in the $x$ th coordinate by $\pi_{x}$, we denote $f \in C_{p}(X)$ such that $f \equiv 0$ as $\mathbf{0}$ and let, for each $\left.n \in \omega, I_{n}=\right]-\frac{1}{n+1}, \frac{1}{n+1}[$.

Proposition 5.7.2. Given $f \in C_{p}(X), \varepsilon>0$ and a finite $F \subset X$, let

$$
B(f, \varepsilon, F)=\left\{g \in C_{p}(x):|f(x)-g(x)|<\varepsilon \text { for all } x \in F\right\} .
$$

Then the following collection is a local basis at $f$ :

$$
\mathscr{B}_{f}=\left\{B\left(f, \frac{1}{n+1}, F\right): n \in \omega, F \in[X]^{<\omega}\right\}
$$

Proof. Exercise 5.7.12

The following lemma can be found, implicitly, in [Sakai 1988]:

Lemma 5.7.3. Given a $T_{3 \frac{1}{2}}$ space $X$,

(a) if $\mathscr{U} \in \Omega$, then

$$
A(\mathscr{U})=\left\{f \in C_{p}(X): \text { for some } U \in \mathscr{U}, f(x)=1 \text { for all } x \in X \backslash U\right\} \in \Omega_{0} ;
$$

(b) if $A \in \Omega_{0}, n \in \omega$ and $\mathscr{U}(A, n)=\left\{f^{-1}\left(I_{n}\right): f \in A\right\} \in \Omega$, then either $X \in \mathscr{U}(A, n)$, or else $\mathscr{U}(A, n) \in \Omega$.

Proof. Suppose $\mathscr{U} \in \Omega$, let $A(\mathscr{U})$ be as in (a) and fix $n \in \omega$ and $F \subset X$ finite. Since $\mathscr{U} \in \Omega$, there is a $U \in \mathscr{U}$ such that $F \subset U$. On the other hand, since $X$ is a $T_{3 \frac{1}{2}}$ space, there is a continuos $f: X \rightarrow \mathbb{R}$ such that $f(x)=0$ for all $x \in F$ and $f(y)=1$ for all $y \in X \backslash U$. Note that $f \in A(\mathscr{U}) \cap B\left(\mathbf{0}, \frac{1}{n+1}, F\right)$, as desired.

On the other hand, suppose $A \in \Omega_{0}$, let $\mathscr{U}(A, n)$ be as in (b) and fix a finite $F \subset X$. Since $A \in \Omega_{\mathbf{0}}$, there is an $f \in A \cap B\left(\mathbf{0}, \frac{1}{n+1}, F\right)$, so $F \subset f^{-1}\left(I_{n}\right)$, which concludes the proof.

Lemma 5.7.4. If $\mathscr{U} \in \Omega$, then $\{U \in \mathscr{U}: F \subset U\}$ is infinite for every finite $F \subset X$.

Proof. Fix a finite $F \subset X$. Since $\mathscr{U} \in \Omega$, there is a $U_{0} \in \mathscr{U}$ such that $F \subset U_{0}$. On the other hand, since $X \notin \mathscr{U}$, there is an $x_{1} \in X$ such that $x_{1} \notin U_{0}$. It follows that there is a $U_{1} \in \mathscr{U}$ such that $F \cup\left\{x_{1}\right\} \subset U_{1}$, which implies that $U_{0} \neq U_{1}$. By proceeding in this manner we inductively find a sequence $\left\langle U_{n}: n \in \omega\right\rangle$ of distinct elements of $\mathscr{U}$ such that $F \subset U_{n}$ for all $n \in \omega$, as desired.

Theorem 5.7.5 ([Scheepers 1997]). On every space $X, \mathrm{~S}_{\mathrm{fin}}(\Omega, \Omega)$ holds if, and only if, ALICE $\Varangle \mathrm{G}_{\mathrm{fin}}(\Omega, \Omega)$. 
Proof. By Proposition 4.0.9,

$$
\operatorname{ALICE} \Varangle \mathrm{G}_{\mathrm{fin}}(\Omega, \Omega) \Longrightarrow \mathrm{S}_{\mathrm{fin}}(\Omega, \Omega)
$$

so suppose $\mathrm{S}_{\text {fin }}(\Omega, \Omega)$ holds and fix a strategy $\gamma$ for ALICE in $\mathrm{G}_{\text {fin }}(\Omega, \Omega)$. By Theorem 3.3.49, $\mathrm{S}_{\text {fin }}(\mathscr{O}, \mathscr{O})$ holds on $X^{k}$ for every $k \in \mathbb{N}$. In this case, by Proposition 3.3.3, $\mathrm{S}_{\text {fin }}(\mathscr{O}, \mathscr{O})$ holds on the disjoint union $Y=\bigcup_{k \in \mathbb{N}} X^{k}$, which, in view of Theorem 4.1.12, implies that $\operatorname{ALICE} \Varangle \mathrm{G}_{\text {fin }}(\mathscr{O}, \mathscr{O})$ on $Y$. We define a strategy $\gamma^{\prime}$ for ALICE in $\mathrm{G}_{\mathrm{fin}}(\mathscr{O}, \mathscr{O})$ over $\mathrm{Y}$ as follows:

- First, let

$$
\gamma^{\prime}(\langle\rangle)=\mathscr{U}_{0}=\bigcup_{k \in \mathbb{N}}\left\{V^{k}: V \in \gamma(\langle\rangle)\right\}
$$

- if Bов answers with $\mathscr{F}_{0}^{\prime}=\left\{V_{0}^{k_{0}}, \ldots, V_{m}^{k_{m}}\right\} \subset \gamma^{\prime}(\langle\rangle)$, let $\mathscr{F}_{0}=\left\{V_{0}, \ldots, V_{m}\right\}$ and then

$$
\gamma^{\prime}\left(\left\langle\mathscr{F}_{0}^{\prime}\right\rangle\right)=\bigcup_{k \in \mathbb{N}}\left\{V^{k}: V \in \gamma\left(\left\langle\mathscr{F}_{0}\right\rangle\right)\right\}
$$

- and so on.

Since $\operatorname{ALICE}_{\chi} \mathrm{G}_{\text {fin }}(\mathscr{O}, \mathscr{O})$ over $Y$, there is a run $\left\langle\gamma^{\prime}(\langle\rangle), \mathscr{F}_{0}^{\prime}, \ldots \gamma^{\prime}\left(\left\langle\mathscr{F}_{0}^{\prime}, \ldots, \mathscr{F}_{n}^{\prime}\right\rangle\right), \mathscr{F}_{n+1}^{\prime}, \ldots\right\rangle$ such that $\bigcup_{n \in \omega} \mathscr{F}_{n}^{\prime}$ covers $Y$. For each $n \in \omega$, let $\mathscr{F}_{n}=\left\{V_{0}, \ldots, V_{m}\right\}$ be such that $\mathscr{F}_{n}^{\prime}=\left\{V_{0}^{k_{0}}, \ldots, V_{m}^{k_{m}}\right\}$ for some $k_{0}, \ldots, k_{m} \in \mathbb{N}$. Then it is clear that ALICE loses the run

$$
\left\langle\gamma(\langle\rangle), \mathscr{F}_{0}, \ldots \gamma\left(\left\langle\mathscr{F}_{0}, \ldots, \mathscr{F}_{n}\right\rangle\right), \mathscr{F}_{n+1}, \ldots\right\rangle
$$

and, therefore, $\gamma$ is not a winning strategy, which concludes the proof.

Theorem 5.7.6 ([Arhangel'skii 1986], [Scheepers 1997]). Let X be a $T_{3 \frac{1}{2}}$ space. Then the following statements are equivalent:

(a) $\mathrm{S}_{\text {fin }}(\Omega, \Omega)$ holds on $X$;

(b) $\operatorname{ALICE} \Varangle \mathrm{G}_{\text {fin }}(\Omega, \Omega)$ on $X$;

(c) ALICE $\Varangle \mathrm{G}_{\mathrm{fin}}\left(\Omega_{\mathbf{0}}, \Omega_{\mathbf{0}}\right)$ on $C_{p}(X)$;

(d) $\mathrm{S}_{\text {fin }}\left(\Omega_{0}, \Omega_{0}\right)$ holds on $C_{p}(X)$.

Proof. Note that the implication (a) $\Longrightarrow$ (b) follows directly from Theorem 5.7.5.

In order to show that $(b) \Longrightarrow(c)$, Let $\gamma$ be a strategy for ALICE in $G_{\text {fin }}\left(\Omega_{0}, \Omega_{0}\right)$. We construct a stratey $\gamma^{\prime}$ for ALICE in $\mathrm{G}_{\mathrm{fin}}(\Omega, \Omega)$ as follows:

- First, fix $\mathscr{U} \in \Omega$. Then, if $\gamma(\langle\rangle)=A_{0}$ and $X \notin \mathscr{U}\left(A_{0}, 0\right)$, let $\gamma^{\prime}(\langle\rangle)=\mathscr{U}\left(A_{0}, 0\right)$. Otherwise, let $\gamma^{\prime}(\langle\rangle)=\mathscr{U}$. 
- When Вов responds with $\mathscr{F}_{0}^{\prime} \subset \gamma^{\prime}(\langle\rangle)$, we have two cases to consider: if $X \notin \mathscr{U}\left(A_{0}, 0\right)$, let $\mathscr{F}_{0} \subset A_{0}$ be such that $\left\{f^{-1}\left(I_{0}\right): f \in \mathscr{F}_{0}\right\}=\mathscr{F}_{0}^{\prime}$. Otherwise, let $\mathscr{F}_{0}=\left\{f_{0}\right\}$, with $f_{0} \in A_{0}$ such that $f_{0}^{-1}\left(I_{0}\right)=X$. Set $\gamma\left(\left\langle\mathscr{F}_{0}\right\rangle\right)=A_{1}$ and then, again, we have two cases to consider: if $X \notin \mathscr{U}\left(A_{1}, 1\right)$, let $\gamma^{\prime}\left(\left\langle\mathscr{F}_{0}^{\prime}\right\rangle\right)=\mathscr{U}\left(A_{1}, 1\right)$. Otherwise, let $\gamma^{\prime}\left(\left\langle\mathscr{F}_{0}^{\prime}\right\rangle\right)=\mathscr{U}$.

- When Вов responds with $\mathscr{F}_{1}^{\prime} \subset \gamma^{\prime}\left(\left\langle\mathscr{F}_{0}^{\prime}\right\rangle\right)$, we have two cases to consider: if $X \notin \mathscr{U}\left(A_{1}, 1\right)$, let $\mathscr{F}_{1} \subset A_{1}$ be such that $\left\{f^{-1}\left(I_{1}\right): f \in \mathscr{F}_{1}\right\}=\mathscr{F}_{1}^{\prime}$. Otherwise, let $\mathscr{F}_{1}=\left\{f_{1}\right\}$, with $f_{1} \in A_{1}$ such that $f_{1}^{-1}\left(I_{1}\right)=X$. Set $\gamma\left(\left\langle\mathscr{F}_{0}, \mathscr{F}_{1}\right\rangle\right)=A_{2}$ and then, again, we have two cases to consider: if $X \notin \mathscr{U}\left(A_{2}, 2\right)$, let $\gamma^{\prime}\left(\left\langle\mathscr{F}_{0}^{\prime}, \mathscr{F}_{1}^{\prime}\right\rangle\right)=\mathscr{U}\left(A_{2}, 2\right)$. Otherwise, let $\gamma^{\prime}\left(\left\langle\mathscr{F}_{0}^{\prime}, \mathscr{F}_{1}^{\prime}\right\rangle\right)=$ $\mathscr{U}$.

- And so on.

Since ALICE $\chi \mathrm{G}_{\text {fin }}(\Omega, \Omega)$, there is a run $\left\langle\gamma^{\prime}(\langle\rangle), \mathscr{F}_{0}^{\prime}, \ldots \gamma^{\prime}\left(\left\langle\mathscr{F}_{0}^{\prime}, \ldots, \mathscr{F}_{n}^{\prime}\right\rangle\right), \mathscr{F}_{n+1}^{\prime}, \ldots\right\rangle$ such that $\bigcup_{n \in \omega} \mathscr{F}_{n}^{\prime} \in \Omega$. Note that if $X \in \mathscr{U}\left(A_{n}, n\right)$ for infinitely many $n \in \omega$, ALICE loses the run with $\gamma$, so assume that there is an $m \in \omega$ such that $X \notin \mathscr{U}\left(A_{n}, n\right)$ for every $n \geq m$. We will show that ALICE loses the run $\left\langle\gamma(\langle\rangle), \mathscr{F}_{0}, \ldots \gamma\left(\left\langle\mathscr{F}_{0}, \ldots, \mathscr{F}_{n}\right\rangle\right), \mathscr{F}_{n+1}, \ldots\right\rangle$, which concludes the proof. Indeed, fix a finite $F \subset X$ and $k \geq m$. By Lemma 5.7.4, $\bigcup_{n \geq k} \mathscr{F}_{n}^{\prime} \in \Omega$, so there is an $n \geq k$ and an $f_{n} \in \mathscr{F}_{n}$ such that $F \subset f_{n}^{-1}\left(I_{n}\right)$. Then $f_{n} \in B\left(\mathbf{0}, \frac{1}{k+1}, F\right) \cap \bigcup_{n \geq k} \mathscr{F}_{n}$ and the proof is complete.

The implication $(\mathrm{c}) \Longrightarrow(\mathrm{d})$ follows directly from Proposition 4.0.9.

Finally, in order to show that $(\mathrm{d}) \Longrightarrow(\mathrm{a})$, suppose $\mathrm{S}_{\text {fin }}\left(\Omega_{\mathbf{0}}, \Omega_{\mathbf{0}}\right)$ holds on $C_{p}(X)$ and let $\left\langle\mathscr{U}_{n}: n \in \omega\right\rangle$ be a sequence of $\omega$-covers for $X$. For each $n \in \omega$, let $A_{n}=A\left(\mathscr{U}_{n}\right)$ and then apply $\mathrm{S}_{\text {fin }}\left(\Omega_{\mathbf{0}}, \Omega_{\mathbf{0}}\right)$ to the sequence $\left\langle A_{n}: n \in \omega\right\rangle$ to find a sequence $\left\langle\mathscr{F}_{n}^{\prime}: n \in \omega\right\rangle$ such that $\mathscr{F}_{n}^{\prime}$ is a finite subset of $A_{n}$ for every $n \in \omega$ and $\bigcup_{n \in \omega} \mathscr{F}_{n}^{\prime} \in \Omega_{0}$. For each $n \in \omega$, let $\mathscr{F}_{n} \subset \mathscr{U}_{n}$ be a finite set such that for each $f \in \mathscr{F}_{n}^{\prime}$ there is a $U_{f} \in \mathscr{F}_{n}$ with $f(x)=1$ for every $x \in X \backslash U_{f}$. To see that $\bigcup_{n \in \omega} \mathscr{F}_{n} \in \Omega$, fix a finite $F \subset X$. Since $\bigcup_{n \in \omega} \mathscr{F}_{n}^{\prime} \in \Omega_{\mathbf{0}}$, there is an $n \in \omega$ and an $f \in \mathscr{F}_{n}^{\prime}$ such that $f \in B(\mathbf{0}, 1, F)$. Then $f(x)<1$ for every $x \in F$, which implies that $F \subset U_{f} \in \mathscr{F}_{n}$ and the proof is complete.

Theorem 5.7.7 ([Scheepers 2014]). Let $X$ be a $T_{3 \frac{1}{2}}$ space. Then $\mathrm{BoB} \uparrow \mathrm{G}_{\mathrm{fin}}(\Omega, \Omega)$ on $X$ if, and only if, $\mathrm{BoB} \uparrow \mathrm{G}_{\mathrm{fin}}\left(\Omega_{\mathbf{0}}, \Omega_{\mathbf{0}}\right)$ on $C_{p}(X)$.

Proof. Suppose $\sigma$ is a winning strategy for $\operatorname{BoB}$ in $\mathrm{G}_{\text {fin }}(\Omega, \Omega)$ over $X$ and fix $\mathscr{U} \in \Omega$. We define a strategy $\sigma^{\prime}$ for Вов in $\mathrm{G}_{\mathrm{fin}}\left(\Omega_{\mathbf{0}}, \Omega_{\mathbf{0}}\right)$ over $C_{p}(X)$ as follows:

- When AliCE plays $A_{0} \in \Omega_{0}$ in the first inning and $X \notin \mathscr{U}\left(A_{0}, 0\right)$, we set $\mathscr{U}_{0}=\mathscr{U}\left(A_{0}, 0\right)$ and $\sigma^{\prime}\left(\left\langle A_{0}\right\rangle\right)=\mathscr{F}_{0}$ such that $\left\{f^{-1}\left(I_{0}\right): f \in \mathscr{F}_{0}\right\}=\sigma\left(\left\langle\mathscr{U}_{0}\right\rangle\right)$. Otherwise, let $\mathscr{U}_{0}=\mathscr{U}$ and $\sigma^{\prime}\left(\left\langle A_{0}\right\rangle\right)=\{f\}$, with $f \in A_{0}$ such that $f^{-1}\left(I_{0}\right)=X$.

- When Alice plays $A_{n} \in \Omega_{0}$ in the $n$th inning and $X \notin \mathscr{U}\left(A_{n}, n\right)$, we set $\mathscr{U}_{n}=\mathscr{U}\left(A_{n}, n\right)$ and $\sigma^{\prime}\left(\left\langle A_{0}, \ldots, A_{n}\right\rangle\right)=\mathscr{F}_{n}$ such that $\left\{f^{-1}\left(I_{n}\right): f \in \mathscr{F}_{n}\right\}=\sigma\left(\left\langle\mathscr{U}_{0}, \ldots, \mathscr{U}_{n}\right\rangle\right)$. Otherwise, let $\mathscr{U}_{n}=\mathscr{U}$ and $\sigma^{\prime}\left(\left\langle A_{0}, \ldots, A_{n}\right\rangle\right)=\{f\}$, with $f \in A_{n}$ such that $f^{-1}\left(I_{n}\right)=X$. 
To see that $\sigma^{\prime}$ is a winning strategy, first of all, note that if $X \in \mathscr{U}\left(A_{n}, n\right)$ for infinitely many $n \in \omega$, then Вов wins the run. So suppose, without loss of generality, that $X \notin \mathscr{U}\left(A_{n}, n\right)$ for all $n \in \omega$. Fix $k \in \omega$ and a finite set $F \subset X$. Since $\sigma$ is a winning strategy, $\bigcup_{n \in \omega} \sigma\left(\left\langle\mathscr{U}_{0}, \ldots, \mathscr{U}_{n}\right\rangle\right) \in \Omega$, so, by Lemma 5.7.4, $\bigcup_{n>k} \sigma\left(\left\langle\mathscr{U}_{0}, \ldots, \mathscr{U}_{n}\right\rangle\right) \in \Omega$. In this case, there is an $n>k$ such that $F \subset U_{n}$ for some $U_{n} \in \sigma\left(\left\langle\mathscr{U}_{0}, \ldots, \mathscr{U}_{n}\right\rangle\right)$. Let $f_{n} \in \mathscr{F}_{n}$ be such that $f_{n}^{-1}\left(I_{n}\right)=U_{n}$. Then $f_{n} \in B\left(\mathbf{0}, \frac{1}{k+1}, F\right)$, as desired. It follows that $\sigma^{\prime}$ is a winning strategy.

Now, suppose that $\sigma$ is a winning strategy for Вов in $\mathrm{G}_{\mathrm{fin}}\left(\Omega_{\mathbf{0}}, \Omega_{\mathbf{0}}\right)$ on $C_{p}(X)$. We define a strategy $\sigma^{\prime}$ for Bов in $\mathrm{G}_{\mathrm{fin}}(\Omega, \Omega)$ on $X$ as follows:

- When ALICE chooses $\mathscr{U}_{0} \in \Omega$ in the first inning, let $A_{0}=A\left(\mathscr{U}_{0}\right)$ and then let $\sigma^{\prime}\left(\left\langle\mathscr{U}_{0}\right\rangle\right)=$ $\mathscr{F}_{0}$ such that for each $f \in \sigma\left(\left\langle A_{0}\right\rangle\right)$ there is a $U \in \mathscr{F}_{0}$ with $f(x)=1$ for all $x \in X \backslash U$.

- When ALICE chooses $\mathscr{U}_{n} \in \Omega$ in the $n$th inning, let $A_{n}=A\left(\mathscr{U}_{n}\right)$ and then let $\sigma^{\prime}\left(\left\langle\mathscr{U}_{0}, \ldots, \mathscr{U}_{n}\right\rangle\right)=$ $\mathscr{F}_{n}$ such that for each $f \in \sigma\left(\left\langle A_{0}, \ldots, A_{n}\right\rangle\right)$ there is a $U \in \mathscr{F}_{n}$ with $f(x)=1$ for all $x \in X \backslash U$.

To see that $\sigma^{\prime}$ is a winning strategy, fix a finite $F \subset X$. Then, since $\sigma$ is a winning strategy, there is an $n \in \omega$ and an $f \in \sigma\left(\left\langle A_{0}, \ldots, A_{n}\right\rangle\right)$ such that $f \in B(\mathbf{0}, 1, F)$. In this case, if $U \in \mathscr{F}_{n}$ is such that $f(x)=1$ for all $x \in X \backslash U$, then $F \subset U$, and the proof is complete.

Following the steps of the proofs of Theorems 5.7.5, 5.7.6 and 5.7.7, one can also show (Exercise 5.7.13):

Theorem 5.7.8 ([Scheepers 1997]). On every space $X, \mathrm{~S}_{1}(\Omega, \Omega)$ holds if, and only if, $\operatorname{ALICE}_{\chi} \mathrm{G}_{1}(\Omega, \Omega)$.

Theorem 5.7.9 ([Sakai 1988], [Scheepers 1997]). Let X be a $T_{3 \frac{1}{2}}$ space. Then the following statements are equivalent:

(a) $\mathrm{S}_{1}(\Omega, \Omega)$ holds on $X$;

(b) $\operatorname{ALICE}_{\chi} \mathrm{G}_{1}(\Omega, \Omega)$ on $X$;

(c) ALICE $\nmid G_{1}\left(\Omega_{0}, \Omega_{0}\right)$ on $C_{p}(X)$;

(d) $\mathrm{S}_{1}\left(\Omega_{\mathbf{0}}, \Omega_{0}\right)$ holds on $C_{p}(X)$.

Theorem 5.7.10 ([Scheepers 2014]). Let $X$ be a $T_{3 \frac{1}{2}}$ space. Then $\mathrm{BoB} \uparrow \mathrm{G}_{1}(\Omega, \Omega)$ on $X$ if, and only if, $\mathrm{BoB} \uparrow \mathrm{G}_{1}\left(\Omega_{\mathbf{0}}, \Omega_{\mathbf{0}}\right)$ on $C_{p}(X)$.

Then, finally, we get:

Corollary 5.7.11. There is a space $X$ with $x \in X$ (namely, $C_{p}\left(2^{\omega}\right)$ with $x=0$ ) such that $\mathrm{S}_{1}\left(\Omega_{x}, \Omega_{x}\right)$ does not hold, but $\mathrm{S}_{\mathrm{fin}}\left(\Omega_{x}, \Omega_{x}\right)$ holds. 
Proof. Note that $\left(2^{\omega}\right)^{k}$ is compact for every $k \in \mathbb{N}$, hence, by Theorem 3.3.49, $\mathrm{S}_{\text {fin }}(\Omega, \Omega)$ holds on $2^{\omega}$.

On the other hand, $S_{1}(\mathscr{O}, \mathscr{O})$ does not hold on $2^{\omega}$, so, in view of Theorem 3.3.48, $\mathrm{S}_{1}(\Omega, \Omega)$ also does not hold.

The result then follows from Theorems 5.7.6 and 5.7.9.

Exercise 5.7.12. Write the details of Proposition 5.7.2's proof.

Exercise 5.7.13. Write the details of Theorems 5.7.8, 5.7.9 and 5.7.10's proofs. 
CHAPTER 6

DIAGRAMS

In this chapter we present some diagrams summarizing the main results displayed along the book. Arrows represent implications. The number to the left of/above an arrow tells us where is the proof of such implication (if it is not obvious) and the number between parenthesis immediately next to it points out to the counterexample of its converse implication. Indications such as "metric" or " $T_{1}$ " next to an arrow tell us that this assumption was required in the specified proof and the number between parenthesis next to this indication points out to the counterexample showing that without said assumption the implication would fail. For simplicity's sake, we will denote "ALICE" by "A" and "Bов" by "B", write "productively" as "prod.", "neighborhood" as "nbhd" and "convergence" as "conv.".

We dedicate:

- Figure 1 to the Banach-Mazur game

- Figure 2 to the showcase the Scheepers Diagram with the nontrivial implications explicitly indicated;

- Figure 3 for the remaining important covering properties

- Figure 4 for the tightness properties. 


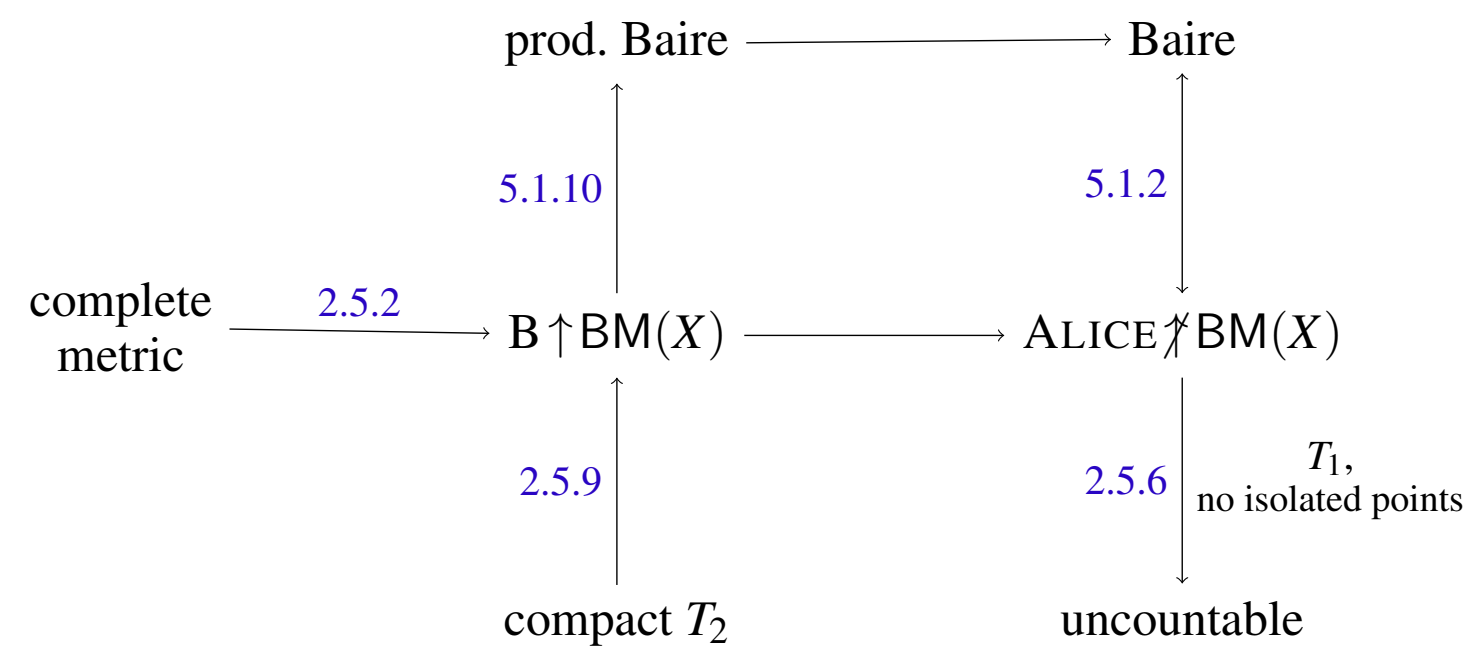

Figure 1 - Banach-Mazur game

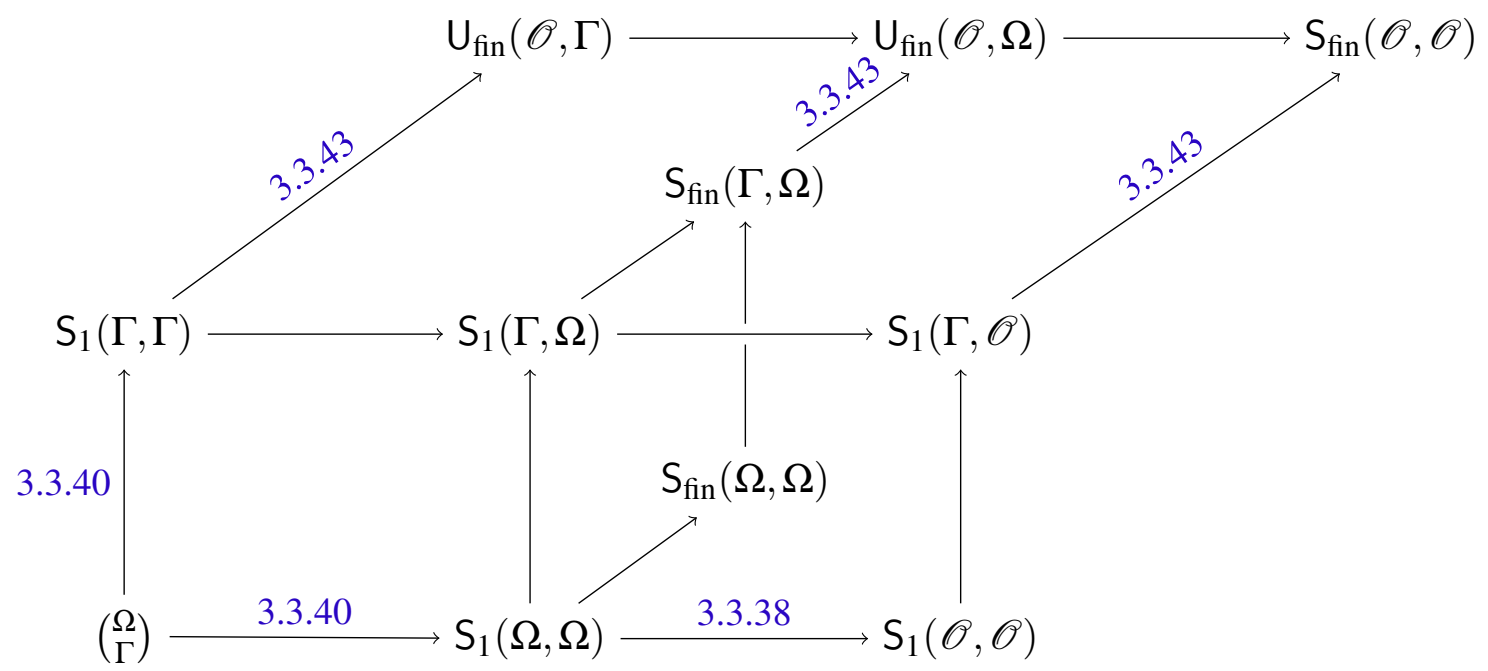

Figure 2 - Scheepers Diagram 


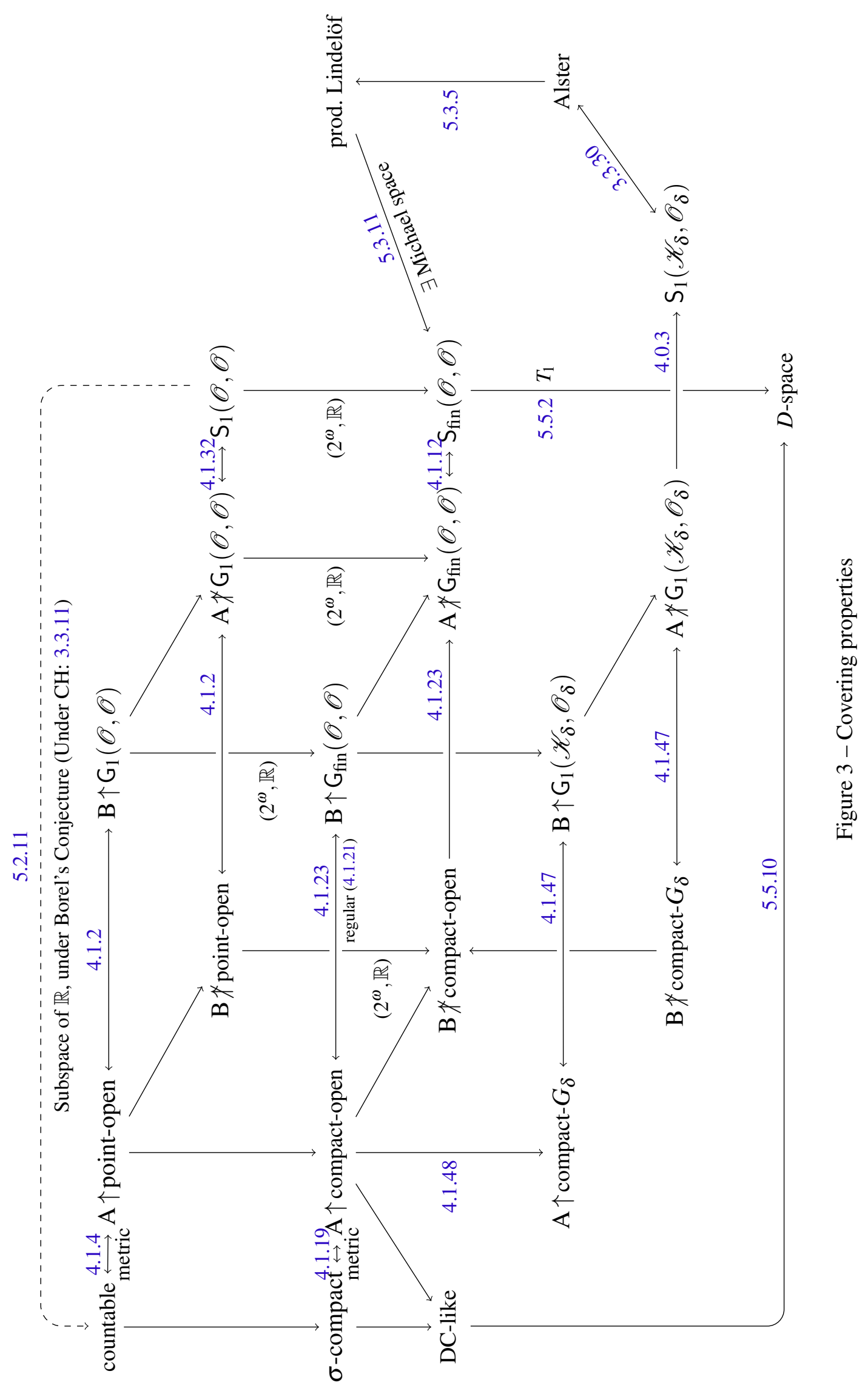




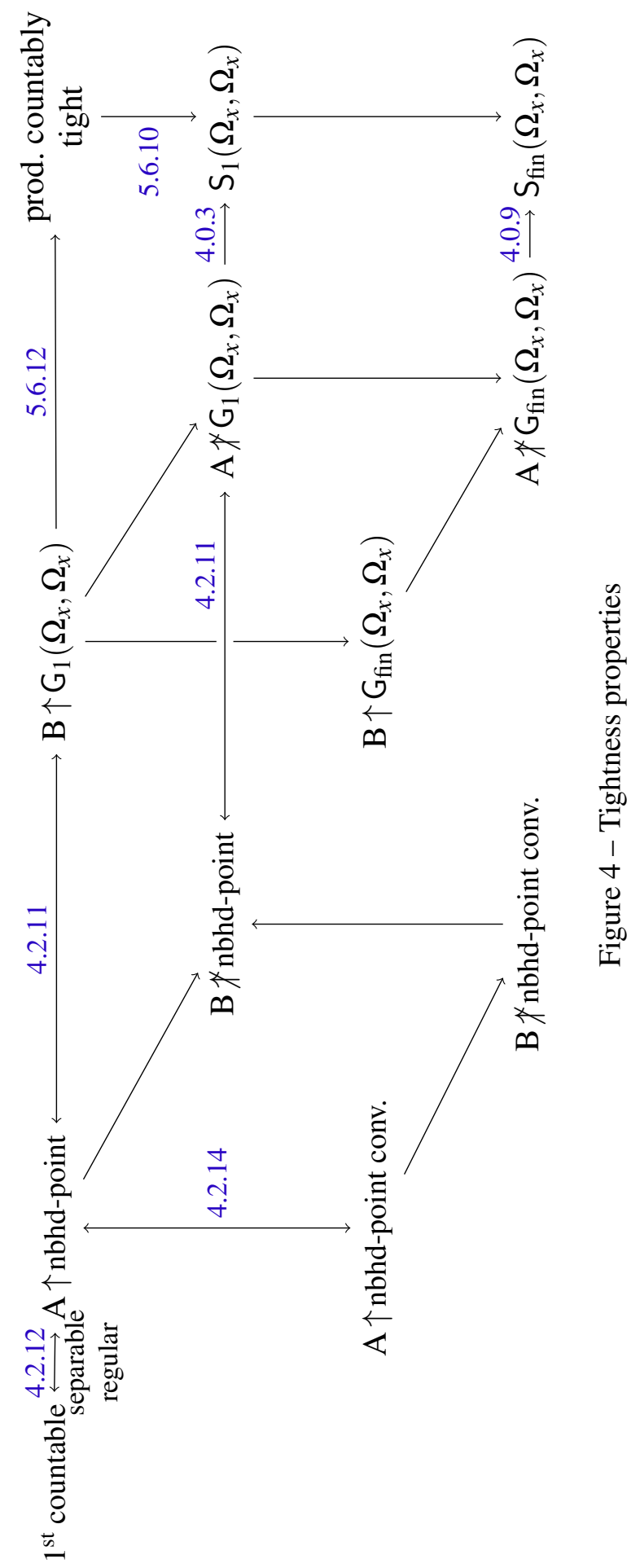


Part II

Results 

CHAPTER

\section{7}

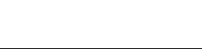

\section{POSITIONAL STRATEGIES AND A PROBLEM IN FINITE COMBINATORICS}

In Section 2.4 of the book we presented the concept of positional strategies and, as already mentioned, we have obtained Theorem 2.4.8 as a small generalization of the main theorem from [Galvin and Telgársky 1986] (there, the kind of game considered was clearly positional for both players) - this generalizations came naturally due to the formalization for games we decided to use throughout the book.

We had initially thought that a corollary to this result would be that ALICE having a winning strategy in the point-open game would imply that she would have a positional winning strategy - but then we soon realized a flaw on our reasoning and, hence, started a hunt for a counterexample. It came as a great surprise that the discrete space of merely 5 points already sufficed as this counterexample (see 2.4.12). The natural question that came after such discover was whether this was the minimum amount of points necessary for ALICE not having a positional winning strategy in this game - and the answer easily came as a yes (which is why it was left as Exercise 2.4.13).

But what if we change the rules of the point-open game to allow ALICE to choose at most $n$ points, with $n \in \mathbb{N}$ (we will call this variation the $n$ points-open game, for now)? Then we have the following:

Proposition 7.0.1. Given $n \in \mathbb{N}$, ALICE has a positional winning strategy in the $n$ points-open game on the discrete space $X=3 n+1$.

Proof. First, fix any $A_{0} \subset X$ such that $\left|A_{0}\right|=n$ and then set $\gamma(\langle\rangle)=A_{0}$. Let $C=X \backslash A_{0}$. Let $\mathscr{F}=\left\{B \subset X:\left|B \cap B_{0}\right|=n\right\}$ (note that this is a finite set) and then fix an enumeration $\mathscr{F}=$ 
$\left\{B_{i}: i \leq m\right\}$. We then set

$$
f(B)=\left\{\begin{array}{l}
B_{i+1 \bmod m}, \text { if } B=B_{i} \text { for some } i \leq m ; \\
B_{0} \cap C, \text { if }|B \cap C|<n ; \\
X \backslash\left(B \cup A_{0}\right), \text { otherwise. }
\end{array}\right.
$$

Then it is clear that if we proceed to recursively define $\gamma$ as $\gamma\left(s^{\wedge} B\right)=f(B)$ for each $s \in \operatorname{dom}(\gamma)$, then it is a positional winning strategy.

Proposition 7.0.2. Given $n \in \mathbb{N}$, ALICE has no positional winning strategy in the $n$ points-open game over a $T_{1}$ space with at least $n+\left(n^{2}+1\right)(n+1)$ points.

Proof. Let $\gamma$ be a positional strategy for ALICE in the discrete space $X=n+\left(n^{2}+1\right)(n+1)$, fix $A_{0}=\gamma(\langle\rangle)$ and $Y=X \backslash A_{0}$, so that $|Y| \geq\left(n^{2}+1\right)(n+1)$. Let $\left\{F_{i} \subset X: 1 \leq i \leq n+1\right\}$ be such that

- $F_{i} \cap F_{j}=A_{0}$ for all distinct $i, j \leq n+1$;

- $\bigcup_{i \leq n+1} F_{i}=X$;

- $\left|F_{i}\right| \geq n^{2}+1$.

For each $i \leq n+1$, let

$$
B_{i}=\bigcup_{\substack{j \leq n+1 \\ j \neq i}} F_{j},
$$

so that $\left|B_{i} \cap Y\right| \leq n\left(n^{2}+1\right)$ and for every $A \subset X$ such that $|A| \leq n, A \subset B_{i}$ for some $i \leq n+1$. Note that $A_{0} \subset B_{i}$ for every $i \leq n+1$, so $\left\langle B_{i}\right\rangle \in \operatorname{dom} \gamma$. In this case, set $C=A_{0} \cup \bigcup_{i \leq n+1} \gamma\left(\left\langle B_{i}\right\rangle\right)$, so that $\langle C\rangle,\left\langle B_{i}, C\right\rangle \in \operatorname{dom} \gamma$ for every $i \leq n+1$ and $|C \cap Y| \leq n(n+1)$. Let $m \leq n+1$ be such that $\gamma(\langle C\rangle) \subset B_{m}$. We may assume $\gamma\left(\left\langle B_{i}\right\rangle\right)$ is not contained in $B_{i}$ itself for every $i \leq n+1$ (otherwise, $\gamma$ would lose the run on which ВоB responds with $B_{i}$ in every inning), so $\left|C \cap B_{m}\right| \geq n$. Then

$$
\left\langle\gamma(\langle\rangle), B_{m}, \gamma\left(\left\langle B_{m}\right\rangle\right), C, \gamma(\langle C\rangle), B_{m}, \gamma\left(\left\langle B_{m}\right\rangle\right), C, \ldots\right\rangle
$$

is a run compatible with $\gamma$. To see that it does not cover $X$, note that

$$
\begin{aligned}
\left|B_{m} \cup C\right| & =\left|A_{0}\right|+|C \cap Y|+\left|B_{m} \cap Y\right|-\left|C \cap B_{m} \cap Y\right| \\
& \leq n+n(n+1)+n\left(n^{2}+1\right)-n \\
& =n+n^{2}+n\left(n^{2}+1\right)<|X|,
\end{aligned}
$$

Hence, $\gamma$ is not a winning strategy. 
This raises a question about finite combinatorics for future investigations: given $n \in \mathbb{N}$, what is the minimum $k_{n} \in \mathbb{N}$ such that, over every $T_{1}$ space $X$ with $|X| \geq k_{n}$, ALICE has no positional winning strategy in the $n$ points-open game? By Propositions 7.0.1 and 7.0.2,

$$
3 n+1<k_{n} \leq n+\left(n^{2}+1\right)(n+1) .
$$

We should note that, for $n=1$, Equation (7.1) gives us $k_{1}=5$, as we already knew from Example 2.4.12 and Exercise 2.4.13. 

CHAPTER

8

(1)

\section{TOPOLOGICAL GAMES OF BOUNDED SELECTIONS}

We present in this chapter the content obtained in the (submitted) paper [Aurichi and Duzi 2019].

We have already seen that variations $\mathrm{S}_{\text {fin }}(\mathscr{A}, \mathscr{B})$ and $\mathrm{S}_{1}(\mathscr{A}, \mathscr{B})$ may give rise to different topological properties (see e.g. 3.3.7 and 3.3.8). When we are talking about games, the difference may be even more dramatic: $\mathrm{G}_{\mathrm{k}}(\mathscr{A}, \mathscr{B})$ might be equivalent to $\mathrm{G}_{1}(\mathscr{A}, \mathscr{B})$ in some instances of $\mathscr{A}, \mathscr{B}$, like in the covering case (as shown in 4.1.39), while on others it might not, as in the tightness case (as presented in 4.2.4). So it is natural to ask what other kind of change can be done.

In this paper we give continuity in this study of the fundamental differences between the covering and the tightness cases. In order to do so we introduce a new kind of variation: what changes if each selection is finite but at the end, the size of all selections has to be bounded by a number? We will show that usually this bounded selections are different from the classical ones and that the behavior can also change depending upon the case (covering, tightness) studied, highlighting a few of what appears to be the reasons for this phenomenon. In the covering case, notably, we show some characterizations for the new game and selection principle variations analogous to classical ones and, as a corollary, we present a characterization for metrizable spaces in terms of two subspaces: a compact and a countable (or strong measure zero with respect to every metric that generates the space's topology).

This paper was organized as follows. In Section 8.1 we present the new variation of selection principle and discuss its first relations with some classical selection principles, showing that in the covering case we have a new intermediate property and that in the tightness case the new variation collapses to one of the classical variations.

In Section 8.2 we present the games naturally associated to the new variation, showing that both in the covering and tightness cases we have new games. In particular, we characterize 
the new game in the tightness case in terms of the classical games.

We dedicate Section 8.3 to present yet two other new variations of selection principles that enable us to characterize the covering case.

In Section 8.4 we show a result for the new variations in the covering case that is analogous to the Pawlikowski and Hurewicz theorems, obtaining yet another characterization of the new variation of selection principle. This result, however, could not be obtained as a corollary of the classical ones, so the proof is presented as an adaptation of the proof of the Pawlikowski Theorem, inspired by a simplified version seen in the notes [Szewczak and Tsaban 2019] provided by Szewczak and Tsaban.

We continue to study the covering case in Section 8.5, where we present a duality analogous to the one given by Galvin in [Galvin 1978].

Finally, Section 8.6 is dedicated to present some known results and examples so we can summarize in two diagrams the contrast reflected by these bounded selections between the covering and tightness cases.

The following trivial fact about topological spaces will also be useful for future arguments.

Fact 8.0.1. Let $X$ be a topological space and $p \in X$. If $A$ is such that $p \in \bar{A}$ and $p \notin \overline{\{x\}}$ for every $x \in A$, then $p \in \overline{A \backslash F}$ for every $F \subset A$ finite.

\subsection{Selection Principles}

Consider the following selection principle based on Definitions 3.1.5 and 3.1.6:

Definition 8.1.1. Let $\mathscr{A}, \mathscr{B}$ be families of sets. We say that $\mathrm{S}_{\mathrm{bnd}}(\mathscr{A}, \mathscr{B})$ holds if, for every sequence $\left\langle A_{n}: n \in \omega\right\rangle$ of elements of $\mathscr{A}$ there is a sequence $\left\langle B_{n}: n \in \omega\right\rangle$ and $k \in \mathbb{N}$ with, for every $n \in \omega$,

a. $B_{n} \subset A_{n}$ is finite;

b. $\bigcup_{n \in \omega} B_{n} \in \mathscr{B}$

c. $\left|B_{n}\right| \leq k$.

It is easy to see that Definition 8.1.1 is different from both $S_{1}(\mathscr{A}, \mathscr{B})$ and $\mathrm{S}_{\text {fin }}(\mathscr{A}, \mathscr{B})$ :

Proposition 8.1.2. $\mathrm{S}_{\mathrm{bnd}}(\mathscr{O}, \mathscr{O})$ holds over every compact space, but $\mathrm{S}_{1}(\mathscr{O}, \mathscr{O})$ does not hold over $2^{\omega}$.

Moreover, $\mathrm{S}_{\mathrm{fin}}(\mathscr{O}, \mathscr{O})$ holds over every $\sigma$-compact space, but $\mathrm{S}_{\mathrm{bnd}}(\mathscr{O}, \mathscr{O})$ does not hold over $\mathbb{R}$. 
On the other hand, for some choices of the families $\mathscr{A}$ and $\mathscr{B}$, the new definition may collapse to classical selection principles (the following result is somewhat of a generalization of 3.2.5.

Proposition 8.1.3. Let $(X, \tau)$ be a topological space and $p \in X$. Then the following properties are equivalent:

(1) $\mathrm{S}_{1}\left(\Omega_{p}, \Omega_{p}\right)$;

(2) $\mathrm{S}_{\mathrm{k}}\left(\Omega_{p}, \Omega_{p}\right)$, for every $k \geq 2$;

(3) $\mathrm{S}_{\mathrm{bnd}}\left(\Omega_{p}, \Omega_{p}\right)$.

Proof. The implications (1) $\Longrightarrow(2) \Longrightarrow(3)$ are clear, so suppose $\mathrm{S}_{\mathrm{bnd}}\left(\Omega_{p}, \Omega_{p}\right)$ holds and let $\left\langle A_{n}: n \in \omega\right\rangle$ be a sequence of subsets of $X$ such that $p \in \overline{A_{n}}$ for every $n \in \omega$. Since $S_{\mathrm{bnd}}\left(\Omega_{p}, \Omega_{p}\right)$ holds, there is a sequence $\left\langle B_{n}: n \in \omega\right\rangle$ and $k \in \omega$ with, for every $n \in \omega$,
a. $B_{n} \subset A_{n}$;
b. $p \in \overline{\bigcup_{n \in \omega} B_{n}}$;
c. $\left|B_{n}\right| \leq k$.

Without loss of generality, we may assume that $\left|B_{n}\right|=k$ for every $n \in \omega$ and we write $B_{n}=$ $\left\{b_{n}^{1}, \ldots, b_{n}^{k}\right\}$ for each $n \in \omega$. Now, let $C_{i}=\left\{b_{n}^{i}: n \in \omega\right\}$ for each $i \leq k$.

CLAIM 8.1.4. There is an $i \leq k$ such that $p \in \overline{C_{i}}$.

Proof. Just note that $\bigcup_{n \in \omega} B_{n}=\bigcup_{i \leq k} C_{i}$ and $\overline{\bigcup_{i \leq k} C_{i}}=\bigcup_{i \leq k} \overline{C_{i}}$.

Let $m \leq k$ be such that $p \in \overline{C_{m}}$. Then the sequence $\left\langle b_{n}^{m}: n \in \omega\right\rangle$ witnesses $\mathrm{S}_{1}\left(\Omega_{p}, \Omega_{p}\right)$ and the proof is complete.

But even when the selection principle collapses, we may find new properties when looking into the new associated games.

\subsection{The associated games}

Before presenting the new game variation, it is worth mentioning here another variation of topological games that have been studied throughout the years (see e.g. [García-Ferreira and Tamariz-Mascar and [7]). It goes as it follows: 
Definition 8.2.1. Let $\mathscr{A}, \mathscr{B}$ be nonempty families of sets and $f \in \mathbb{N}^{\omega}$. We denote by $\mathrm{G}_{\mathrm{f}}(\mathscr{A}, \mathscr{B})$ the game, played between AliCE and BoB, in which in each inning $n \in \omega$ ALICE chooses $A_{n} \in \mathscr{A}$ as BОB responds with $B_{n} \subset A_{n}$ such that $\left|B_{n}\right| \leq f(n)$ and $\mathrm{BOB}$ wins if $\bigcup_{n \in \omega} B_{n} \in \mathscr{B}$ (ALICE wins otherwise).

As with the classical selection principles, new associated games naturally arise from the new selection principles.

Definition 8.2.2. Let $\mathscr{A}, \mathscr{B}$ be nonempty families of sets. We denote by $\mathrm{G}_{\mathrm{bnd}}(\mathscr{A}, \mathscr{B})$ the game, played between AlICE and BOB, in which in each inning $n \in \omega$ AlICE chooses $A_{n} \in \mathscr{A}$ as BoB responds with $B_{n} \subset A_{n}$ finite and $\mathrm{BOB}$ wins if there is an $k \in \mathbb{N}$ such that,

a. $\bigcup_{n \in \omega} B_{n} \in \mathscr{B}$

b. $\left|B_{n}\right| \leq k$ for every $n \in \omega$.

Otherwise, we say that ALICE wins.

As with the usual selection principles, we immediately have:

Proposition 8.2.3. Given $\mathscr{A}$ and $\mathscr{B}$ families of sets,

$$
\operatorname{ALICE} \ngtr \mathrm{G}_{\text {bnd }}(\mathscr{A}, \mathscr{B}) \Longrightarrow \mathrm{S}_{\text {bnd }}(\mathscr{A}, \mathscr{B}) \text {. }
$$

The following result will be useful in some arguments.

Lemma 8.2.4. Suppose $\sigma$ is a winning strategy for $\mathrm{B}$ ов in $\mathrm{G}_{\mathrm{bnd}}(\mathscr{A}, \mathscr{B})$. Then, for every $r \in$ $<\omega \mathscr{A}$ there is an $s \in{ }^{<\omega} \mathscr{A}$ and an $m \in \mathbb{N}$ such that $\left|\sigma\left(r^{\urcorner} s^{\urcorner} t\right)\right| \leq m$ for every $t \in{ }^{<\omega} \mathscr{A}$.

Proof. Suppose our thesis is false and let $r \in{ }^{<\omega} \mathscr{A}$ be the sequence witnessing this assertion. Then there is an $s_{1} \in{ }^{<\omega} \mathscr{A}$ such that $\left|\sigma\left(r^{\wedge} s_{1}\right)\right|>1$. Again, we may pick an $s_{2} \in{ }^{<\omega} \mathscr{A}$ such that $\left|\sigma\left(r^{\wedge} s_{1} s_{2}\right)\right|>2$. Suppose we have picked $\left\{s_{i}: i \leq n\right\}$ such that $\left|\sigma\left(r^{\frown} s_{1} \ldots \frown s_{k}\right)\right|>k$ for every $k \leq n$. Then we may pick $s_{n+1} \in<\omega \mathscr{A}$ such that $\left|\sigma\left(r^{\wedge} s_{1} \ldots \frown s_{n}^{\wedge} s_{n+1}\right)\right|>n+1$. We have just defined a sequence $\left\langle s_{n}: n \in \mathbb{N}\right\rangle$ such that $\left|\sigma\left(r^{\wedge} s_{1} \cdots^{\frown} s_{n}\right)\right|>n$ for every $n \in \mathbb{N}$, a contradiction to the fact that $\sigma$ is a winning strategy in $\mathrm{G}_{\text {bnd }}(\mathscr{A}, \mathscr{B})$.

Now, even though Proposition 8.1.3 tells us that $\mathrm{S}_{\mathrm{bnd}}\left(\Omega_{p}, \Omega_{p}\right)$ is not really a new selection principle, the same cannot be said about the game associated to this principle. In order to prove this, let us first characterize the new game in terms of the already known tightness games:

Theorem 8.2.5. AliCE has a winning strategy in $\mathrm{G}_{\mathrm{bnd}}\left(\Omega_{p}, \Omega_{p}\right)$ if, and only if, ALICE has a winning strategy in $\mathrm{G}_{\mathrm{k}}\left(\Omega_{p}, \Omega_{p}\right)$ for every $k \in \mathbb{N}$. 
The idea behind the proof of Theorem 8.2.5 is that, in $\mathrm{G}_{\mathrm{bnd}}\left(\Omega_{p}, \Omega_{p}\right)$, ALICE may pretend, at every inning, that the game just started without losing any relevant information, because, in view of Fact 8.0.1, the finite set of points Вов have chosen thus far is irrelevant to the winning criteria.

So ALICE may start the game playing with a winning strategy in the game $\mathrm{G}_{1}\left(\Omega_{p}, \Omega_{p}\right)$ and, if В ОВ chooses more than one point (say, $k$ points), AlICE may just pretend the game restarted and then proceed to play with a winning strategy in the game $\mathrm{G}_{\mathrm{k}}\left(\Omega_{p}, \Omega_{p}\right)$. If B BB wants to win, he must eventually stop raising the amount of points he chooses, so from that moment on he will be playing against a winning strategy for ALICE in some $\mathrm{G}_{\mathrm{k}}\left(\Omega_{p}, \Omega_{p}\right)$, and will, therefore, lose the game.

Formally:

Proof of Theorem 8.2.5. Given $k \in \mathbb{N}$, the implication

$$
\operatorname{ALICE} \uparrow G_{\text {bnd }}\left(\Omega_{p}, \Omega_{p}\right) \Longrightarrow \operatorname{ALICE} \uparrow G_{\mathrm{k}}\left(\Omega_{p}, \Omega_{p}\right)
$$

is obvious.

So, suppose that for each $k \in \mathbb{N}$ there is a winning strategy $\gamma_{k}$ for ALICE in $\mathrm{G}_{\mathrm{k}}\left(\Omega_{p}, \Omega_{p}\right)$. Then we construct a strategy $\gamma$ for ALICE in $\mathrm{G}_{\mathrm{bnd}}\left(\Omega_{p}, \Omega_{p}\right)$ as it follows. First, let $\gamma(\langle\rangle)=\gamma_{1}(\langle\rangle)$. If BoB chooses $B_{0} \subset \gamma_{1}(\langle\rangle)$ with $\left|B_{0}\right| \leq 1$, then we let $\gamma\left(\left\langle B_{0}\right\rangle\right)=\gamma_{1}\left(\left\langle B_{0}\right\rangle\right)$. Otherwise, if $\left|B_{0}\right|=k_{0}$ for some $k_{0}>1$, let $\gamma\left(\left\langle B_{0}\right\rangle\right)=\gamma_{k_{0}}(\langle\rangle)$. In general, suppose $\gamma$ is defined up to $\left\langle B_{i}: i \leq n\right\rangle$ and that, for each $m \leq n, \gamma\left(\left\langle B_{i}: i \leq m\right\rangle\right)=\gamma_{k_{m}}\left(\left\langle B_{i}: l_{m}<i \leq m\right\rangle\right)$, for some $l_{m} \leq m$ and $k_{m} \in \mathbb{N}$. If BоB chooses $B_{n+1} \subset \gamma\left(\left\langle B_{i}: i \leq n\right\rangle\right)$ such that $\left|B_{n+1}\right| \leq k_{n}$, then we simply put

$$
\gamma\left(\left\langle B_{i}: i \leq n\right\rangle^{\frown} B_{n+1}\right)=\gamma_{k_{n}}\left(\left\langle B_{i}: l_{n}<i \leq n\right\rangle^{\frown} B_{n+1}\right) .
$$

Otherwise, if $\left|B_{n+1}\right|=k_{n+1}>k_{n}$, we let $\gamma\left(\left\langle B_{i}: i \leq n\right\rangle^{\frown} B_{n+1}\right)=\gamma_{k_{n+1}}(\langle\rangle)$.

Suppose B Ов plays $\left\langle B_{n}: n \in \omega\right\rangle$ against $\gamma$ in such a way that, for every $n \in \omega,\left|B_{n}\right| \leq k$ for some (minimal) $k \in \mathbb{N}$. Then there must be an (also minimal) $l \in \omega$ such that $\left|B_{l}\right|=k$ and $\left|B_{n}\right| \leq k$ for every $n \geq l$. Then, by the construction presented here, $\left\langle B_{n}: n \geq l\right\rangle$ is a play against $\gamma_{k}$. Finally, since each one of the $\gamma_{n}$ 's are winning strategies for ALICE, we may apply Fact 8.0.1 to $\bigcup_{n \in \omega} B_{n}$ and conclude that if $p \in \overline{\bigcup_{n \in \omega} B_{n}}$, then $p \in \overline{\bigcup_{n \geq l} B_{n}}$, which would contradict the fact that $\gamma_{k}$ is a winning strategy in $\mathrm{G}_{\mathrm{k}}\left(\Omega_{p}, \Omega_{p}\right)$. It follows that $\gamma$ is indeed a winning strategy for ALICE in $\mathrm{G}_{\mathrm{bnd}}\left(\Omega_{p}, \Omega_{p}\right)$.

Corollary 8.2.6. If $\mathrm{S}_{1}\left(\Omega_{p}, \Omega_{p}\right)$ does not hold, then ALICE has a winning strategy in $\mathrm{G}_{\mathrm{bnd}}\left(\Omega_{p}, \Omega_{p}\right)$.

The following result shows us that there is an $f \in \mathbb{N}^{\omega}$ such that $\mathrm{G}_{\mathrm{bnd}}\left(\Omega_{p}, \Omega_{p}\right)$ is not equivalent to $\mathrm{G}_{\mathrm{f}}\left(\Omega_{p}, \Omega_{p}\right)$.

Proposition 8.2.7 ([García-Ferreira and Tamariz-Mascarúa 1995] - Example 3.7, [7] - Example 3.5). There is a space $X$ with a point $p$ on which $\operatorname{BoB} \uparrow \mathrm{G}_{\mathrm{f}}\left(\Omega_{p}, \Omega_{p}\right)$ for any $f \in \mathbb{N}^{\omega}$ unbounded, but $\mathrm{S}_{1}\left(\Omega_{p}, \Omega_{p}\right)$ fails. 
Corollary 8.2.8. There is a space $X$ with a point $p$ on which $\mathrm{BoB} \uparrow \mathrm{G}_{\mathrm{f}}\left(\Omega_{p}, \Omega_{p}\right)$ (in particular, BoB $\left.\uparrow \mathrm{G}_{\mathrm{fin}}\left(\Omega_{p}, \Omega_{p}\right)\right)$ and $\operatorname{ALICE} \uparrow \mathrm{G}_{\mathrm{bnd}}\left(\Omega_{p}, \Omega_{p}\right)$.

On the other hand, to show that $\mathrm{G}_{\mathrm{bnd}}\left(\Omega_{p}, \Omega_{p}\right)$ is not equivalent to $\mathrm{G}_{\mathrm{k}}\left(\Omega_{p}, \Omega_{p}\right)$ for any $k \in \mathbb{N}$, we just need to consider Proposition 4.2.7:

Proposition 8.2.9. For each $k \in \mathbb{N}$ there is a countable space $X_{k}$ with only one non-isolated point $p_{k}$ on which ALICE $\uparrow \mathrm{G}_{\mathrm{k}}\left(\Omega_{p_{k}}, \Omega_{p_{k}}\right)$, and $\mathrm{BOB} \uparrow \mathrm{G}_{\mathrm{bnd}}\left(\Omega_{p_{k}}, \Omega_{p_{k}}\right)$.

We note that Proposition 4.2.7 gives us examples on which, for each $k \in \omega, \mathrm{BoB} \uparrow \mathrm{G}_{\mathrm{bnd}}\left(\Omega_{p_{k}}, \Omega_{p_{k}}\right)$. But we concluded this because $\mathrm{BOB} \uparrow \mathrm{G}_{\mathrm{k}+1}\left(\Omega_{p_{k}}, \Omega_{p_{k}}\right)$. As the following theorem tells us, this was no coincidence.

Theorem 8.2.10. Вов has a winning strategy in $\mathrm{G}_{\mathrm{bnd}}\left(\Omega_{p}, \Omega_{p}\right)$ if, and only if, there is an $m \in \mathbb{N}$ such that Вов has a winning strategy in $\mathrm{G}_{\mathrm{m}}\left(\Omega_{p}, \Omega_{p}\right)$.

Proof. It is clear that if Вов has a winning strategy in $\mathrm{G}_{\mathrm{m}}\left(\Omega_{p}, \Omega_{p}\right)$ for some $m \in \mathbb{N}$, then Bов has a winning strategy in $\mathrm{G}_{\mathrm{bnd}}\left(\Omega_{p}, \Omega_{p}\right)$.

So, suppose Вов has a winning strategy $\sigma$ in $\mathrm{G}_{\mathrm{bnd}}\left(\Omega_{p}, \Omega_{p}\right)$. Without loss of generality, we may assume that ALICE plays only with sets $A \in \Omega_{p}$ such that $p \notin \overline{\{a\}}$ for every $a \in A$. Let $s \in{ }^{<\omega} \Omega_{p}$ and $m \in \mathbb{N}$ be as in Lemma 8.2.4 for $r=\langle\rangle$. Then we define a strategy $\sigma_{m}$ for BoB in $\mathrm{G}_{\mathrm{m}}\left(\Omega_{p}, \Omega_{p}\right)$ as it follows: for each $t \in{ }^{<\omega} \Omega_{p}$, let $\sigma_{m}(t)=\sigma\left(s^{\frown} t\right)$.

To see that this is a winning strategy, let $\left\langle A_{n}: n \in \omega\right\rangle$ be a sequence of elements of $\Omega_{p}$. By construction, $\left|\sigma_{m}\left(A_{0}, \ldots A_{k}\right)\right| \leq m$ for every $k \in \omega$. Also, since $\sigma$ is a winning strategy, $p \in$ $\overline{\sigma(s \uparrow 1) \cup \cdots \cup \sigma(s) \cup\left(\bigcup_{k \in \omega} \sigma\left(s^{\wedge}\left\langle A_{0}, \ldots, A_{k}\right\rangle\right)\right)}$. Finally, if we apply Fact 8.0.1 to the set $\sigma(s \uparrow$ 1) $\cup \cdots \cup \sigma(s) \cup\left(\bigcup_{k \in \omega} \sigma\left(s^{\wedge}\left\langle A_{0}, \ldots, A_{k}\right\rangle\right)\right)$, we conclude that $p \in \overline{\bigcup_{k \in \omega} \sigma\left(s^{\wedge}\left\langle A_{0}, \ldots, A_{k}\right\rangle\right)}=$ $\overline{\bigcup_{k \in \omega} \sigma_{m}\left(\left\langle A_{0}, \ldots, A_{k}\right\rangle\right)}$, and the proof is complete.

We note that the characterizations presented in Theorems 8.2.5 and 8.2.10 would still hold if we replace " $\Omega_{p}$ " with " $D$ ", because the key argument used there was that, except for some trivial cases, we can ignore finite innings of the game to check the winning criteria. The same thing cannot be said about $\mathrm{G}_{\mathrm{bnd}}(\mathscr{O}, \mathscr{O})$, because if the game is played over a compact space, for instance, В ОВ may win in the very first inning - but, on the other hand, ALICE has a winning strategy in $\mathrm{G}_{\mathrm{k}}(\mathscr{O}, \mathscr{O})$ over $2^{\omega}$ for every $k \in \mathbb{N}$.

So now we turn our attention to covering games:

Proposition 8.2.11. In every compact space, $\mathrm{BOB} \uparrow \mathrm{G}_{\mathrm{bnd}}(\mathscr{O}, \mathscr{O})$, but $\operatorname{ALICE} \uparrow \mathrm{G}_{1}(\mathscr{O}, \mathscr{O})$ over $2^{\omega}$. Moreover, Bов $\uparrow \mathrm{G}_{\mathrm{fin}}(\mathscr{O}, \mathscr{O})$ over every $\sigma$-compact space, but $\mathrm{ALICE} \uparrow \mathrm{G}_{\mathrm{bnd}}(\mathscr{O}, \mathscr{O})$ over $\mathbb{R}$.

Now suppose $X$ is a space with a compact subset $K$ such that, for every $V \supset K$ open, Вов has a winning strategy in $\mathrm{G}_{1}(\mathscr{O}, \mathscr{O})$ over the complement $X \backslash V$. Clearly, this implies that BoB 
has a winning strategy over $X$ in $\mathrm{G}_{\mathrm{bnd}}(\mathscr{O}, \mathscr{O})$. What is surprising, though, is that the converse actually holds if $X$ is regular. To prove this, however, we take a step back to define some other variations of the classical selection principles and games.

\subsection{The "modfin" and "mod 1" variations}

Consider the following simple variations of the classical selection principles, with their respective associated games.

Definition 8.3.1. Let $f \in \mathbb{N}^{\omega}$, and $\mathscr{A}, \mathscr{B}$ be families of sets. We say that $\mathrm{S}_{\mathrm{f}}(\mathscr{A}, \mathscr{B}) \bmod$ fin holds if, for every sequence $\left\langle A_{n}: n \in \omega\right\rangle$ of elements of $\mathscr{A}$, there is a sequence $\left\langle B_{n}: n \in \omega\right\rangle$, such that,

a. $B_{n} \subset A_{n}$ is finite for every $n \in \omega$;

b. $\bigcup_{n \in \omega} B_{n} \in \mathscr{B}$

c. $\left\{n \in \omega:\left|B_{n}\right|>f(n)\right\}$ is finite.

When there is a $k \in \mathbb{N}$ with $f \equiv k$ we simply write $\mathrm{S}_{\mathrm{k}}(\mathscr{A}, \mathscr{B}) \bmod$ fin instead of $\mathrm{S}_{\mathrm{f}}(\mathscr{A}, \mathscr{B}) \bmod$ fin.

We then define the property $\mathrm{S}_{\mathrm{f}}(\mathscr{A}, \mathscr{B}) \bmod 1$ as $\mathrm{S}_{\mathrm{f}}(\mathscr{A}, \mathscr{B}) \bmod$ fin with condition (c) replaced by " $\left\{n \in \omega:\left|B_{n}\right|>f(n)\right\} \subset\{0\}$ ", that is, " $\left|B_{n}\right| \leq f(n)$ for every $n \geq 1$ ".

Definition 8.3.2. Let $f \in \mathbb{N}^{\omega}$, and $\mathscr{A}, \mathscr{B}$ be families of sets with $\mathscr{A} \neq \emptyset$ and $\emptyset \notin \mathscr{A}$. We denote by $\mathrm{G}_{\mathrm{f}}(\mathscr{A}, \mathscr{B}) \bmod$ fin the game, played between ALICE and BOB, in which in each inning $n \in \omega$ AlICE chooses $A_{n} \in \mathscr{A}$ as BоB responds with $B_{n} \subset A_{n}$ finite and BоB wins if,

a. $\bigcup_{n \in \omega} B_{n} \in \mathscr{B}$

b. $\left\{n \in \omega:\left|B_{n}\right|>f(n)\right\}$ is finite.

When there is a $k \in \mathbb{N}$ with $f \equiv k$ we simply write $\mathrm{G}_{\mathrm{f}}(\mathscr{A}, \mathscr{B}) \bmod$ fin as $\mathrm{G}_{\mathrm{k}}(\mathscr{A}, \mathscr{B}) \bmod$ fin.

We then define the game $\mathrm{G}_{\mathrm{f}}(\mathscr{A}, \mathscr{B}) \bmod 1$ as $\mathrm{G}_{\mathrm{f}}(\mathscr{A}, \mathscr{B}) \bmod$ fin with condition (b) replaced by " $\left\{n \in \omega:\left|B_{n}\right|>f(n)\right\} \subset\{0\}$ " (that is, in other to have a chance of winning the game, Вов may choose more elements then $f$ allows only in the first inning).

Again, as with the usual selection principles, we also have here:

Proposition 8.3.3. Let $f \in \mathbb{N}^{\omega}$, and $\mathscr{A}, \mathscr{B}$ be families of sets. Then the following implications hold

- ALICE $\ngtr \mathrm{G}_{\mathrm{f}}(\mathscr{A}, \mathscr{B}) \bmod$ fin $\Longrightarrow \mathrm{S}_{\mathrm{f}}(\mathscr{A}, \mathscr{B}) \bmod$ fin;

- $\operatorname{ALICE} \ngtr \mathrm{G}_{\mathrm{f}}(\mathscr{A}, \mathscr{B}) \bmod 1 \Longrightarrow \mathrm{S}_{\mathrm{f}}(\mathscr{A}, \mathscr{B}) \bmod 1$. 
In the tightness case, the new selection principles and games collapse to the classical ones:

Proposition 8.3.4. Let $(X, \tau)$ be a topological space and $p \in X$. Then the following properties are equivalent:

(1) $\mathrm{S}_{1}\left(\Omega_{p}, \Omega_{p}\right)$;

(2) $\mathrm{S}_{\mathrm{k}}\left(\Omega_{p}, \Omega_{p}\right)$, for every $k \in \mathbb{N}$;

(3) $\mathrm{S}_{\mathrm{bnd}}\left(\Omega_{p}, \Omega_{p}\right)$;

(4) $\mathrm{S}_{1}\left(\Omega_{p}, \Omega_{p}\right) \operatorname{modfin}$;

(5) $\mathrm{S}_{1}\left(\Omega_{p}, \Omega_{p}\right) \bmod 1$.

Proof. Clearly (1) $\Longrightarrow(4)$ and (1) $\Longrightarrow(5)$. On the other hand, (4) $\Longrightarrow(3)$ and (5) $\Longrightarrow(3)$, so the result follows from Proposition 8.1.3.

Proposition 8.3.5. Let $f \in \mathbb{N}^{\omega}$. Then the following games are equivalent:

(a) $\mathrm{G}_{\mathrm{f}}\left(\Omega_{p}, \Omega_{p}\right)$;

(b) $\mathrm{G}_{\mathrm{f}}\left(\Omega_{p}, \Omega_{p}\right) \bmod 1$;

(c) $\mathrm{G}_{\mathrm{f}}\left(\Omega_{p}, \Omega_{p}\right) \bmod$ fin.

Proof. We will show the result for $f \equiv 1$ (the general case is analogous). The implications

$$
\begin{aligned}
& \operatorname{ALICE} \uparrow G_{1}\left(\Omega_{p}, \Omega_{p}\right) \bmod \text { fin } \Longrightarrow \operatorname{ALICE} \uparrow G_{1}\left(\Omega_{p}, \Omega_{p}\right) \bmod 1 \Longrightarrow \operatorname{ALICE} \uparrow G_{1}\left(\Omega_{p}, \Omega_{p}\right) \\
& \text { Вов } \uparrow \mathrm{G}_{1}\left(\Omega_{p}, \Omega_{p}\right) \Longrightarrow \mathrm{BoB} \uparrow \mathrm{G}_{1}\left(\Omega_{p}, \Omega_{p}\right) \bmod 1 \Longrightarrow \mathrm{BoB} \mathrm{G}_{1}\left(\Omega_{p}, \Omega_{p}\right) \bmod \text { fin }
\end{aligned}
$$
are clear.

Suppose there is a winning strategy $\gamma_{1}$ for ALICE in $\mathrm{G}_{1}\left(\Omega_{p}, \Omega_{p}\right)$. For each sequence $s \in \operatorname{dom}\left(\gamma_{1}\right)$, let $A_{s}=\gamma_{1}(s)$ and then fix a choice function $f_{s}:\left[A_{s}\right]^{<\omega} \rightarrow A_{s}$ (that is, $f_{s}(F) \in F$ for every $F \subset A_{s}$ finite). Now, consider the following strategy $\gamma$ for ALICE in $G_{1}\left(\Omega_{p}, \Omega_{p}\right) \bmod$ fin:

- Let $\gamma(\langle\rangle)=A_{\langle\rangle}$;

- After BoB chooses $B_{0} \subset A_{\langle\rangle}$, let

$$
\gamma\left(\left\langle B_{0}\right\rangle\right)=A_{\left\langle f_{\langle\rangle}\left(B_{0}\right)\right\rangle} ;
$$

- After BoB chooses $B_{1} \subset A_{\left\langle f_{\langle\rangle}\left(B_{0}\right)\right\rangle}$, let

$$
\gamma\left(\left\langle B_{0}, B_{1}\right\rangle\right)=A_{\left\langle f_{\langle\rangle}\left(B_{0}\right), f_{\left\langle B_{0}\right\rangle}\left(B_{1}\right)\right\rangle} ;
$$


- After BoB chooses $B_{2} \subset A_{\left\langle f_{\langle\rangle}\left(B_{0}\right), f_{\left\langle B_{0}\right\rangle}\left(B_{1}\right)\right\rangle}$, let

$$
\gamma\left(\left\langle B_{0}, B_{1}, B_{2}\right\rangle\right)=A_{\left\langle f_{\langle\rangle}\left(B_{0}\right), f_{\left\langle B_{0}\right\rangle}\left(B_{1}\right), f_{\left\langle B_{0}, B_{1}\right\rangle}\left(B_{2}\right)\right\rangle}
$$

- (and so on).

Note that if we assume that $\left\langle B_{n}: n \in \omega\right\rangle$ is a winning play of BoB against $\gamma$, then $\left(\bigcup_{n \in \omega} B_{n}\right) \backslash$ $\left\{f_{\langle\rangle}\left(B_{0}\right), f_{\left\langle B_{0}\right\rangle}\left(B_{1}\right), f_{\left\langle B_{0}, B_{1}\right\rangle}\left(B_{2}\right), \ldots\right\}$ is contained in the finitely many responses of Bов in which he chose more than one point, hence it is finite. But since $\gamma_{1}$ is a winning strategy, $\bigcup_{n \in \omega} B_{n}$ satisfies the hypothesis of Fact 8.0.1, so $\left\langle f_{\langle\rangle}\left(B_{0}\right), f_{\left\langle B_{0}\right\rangle}\left(B_{1}\right), f_{\left\langle B_{0}, B_{1}\right\rangle}\left(B_{2}\right), \ldots\right\rangle$ is a winning play for BOB against $\gamma_{1}$, a contradiction.

Finally, suppose there is a winning strategy $\sigma$ for BоB in $\mathrm{G}_{1}\left(\Omega_{p}, \Omega_{p}\right) \bmod$ fin (we may assume that $\sigma$ always tells Вов to choose nonempty subsets). For each $s \in{ }^{<\omega} \Omega_{p}$, let $B_{s}=\sigma(s)$. If there is an $x \in B_{s}$ such that $p \in \overline{\{x\}}$, fix $b_{s}=x$. Otherwise, fix any $b_{s} \in B_{s}$. Naturally, we define the strategy $\sigma_{1}$ for Вов in $\mathrm{G}_{1}\left(\Omega_{p}, \Omega_{p}\right)$ as $\sigma_{1}(s)=b_{s}$ for every $s \in{ }^{<\omega} \Omega_{p}$.

Now, suppose $\left\langle A_{n}: n \in \omega\right\rangle$ is played by ALICE in $\mathrm{G}_{1}\left(\Omega_{p}, \Omega_{p}\right)$ and let $\left\langle B_{n}: n \in \omega\right\rangle$ and $\left\langle b_{n}: n \in \omega\right\rangle$ be $\sigma$ 's and $\sigma_{1}$ 's, respectively, responses to this play. Since $\sigma$ is a winning strategy,

a. $B=\bigcup_{n \in \omega} B_{n} \in \Omega_{p}$;

b. $\left\{k \in \omega:\left|B_{k}\right|>1\right\}$ is finite.

Then we have two possibilities:

- There is an $x \in B$ such that $p \in \overline{\{x\}}$ : in this case, there is an $n \in \omega$ such that $p \in \overline{\left\{b_{n}\right\}}$, and so $\left\langle b_{n}: n \in \omega\right\rangle$ is a winning play.

- There is no $x \in B$ such that $p \in \overline{\{x\}}$ : Then we apply Fact 8.0.1 to the set $B$ to conclude that $p \in \overline{\left\{b_{n}: n \in \omega\right\}}$, hence $\left\langle b_{n}: n \in \omega\right\rangle$ is a winning play.

It follows that $\sigma_{1}$ is a winning strategy.

This is not the case, however, when we consider $\mathscr{A}=\mathscr{B}=\mathscr{O}$, for instance. Note that Proposition 8.1.2 still holds if we replace " $\mathrm{S}_{\text {bnd }}(\mathscr{O}, \mathscr{O})$ " by " $\mathrm{S}_{1}(\mathscr{O}, \mathscr{O}) \bmod$ fin" or " $\mathrm{S}_{1}(\mathscr{O}, \mathscr{O}) \bmod 1$ ". This is no coincidence, as we will see later. But first, consider the following auxiliary results.

Proposition 8.3.6. For every $f \in \mathbb{N}^{\omega}$,

$$
\mathrm{S}_{\mathrm{f}}(\mathscr{O}, \mathscr{O}) \bmod 1 \Longleftrightarrow \mathrm{S}_{\mathrm{f}}(\mathscr{O}, \mathscr{O}) \bmod \text { fin. }
$$

Proof. The implication

$$
\mathrm{S}_{\mathrm{f}}(\mathscr{O}, \mathscr{O}) \bmod 1 \Longrightarrow \mathrm{S}_{\mathrm{f}}(\mathscr{O}, \mathscr{O}) \bmod \text { fin }
$$


is clear.

Now, suppose $\mathrm{S}_{\mathrm{f}}(\mathscr{O}, \mathscr{O}) \bmod$ fin holds and let $\left\langle\mathscr{U}_{n}: n \in \omega\right\rangle$ be a sequence of open covers. Let $\left\langle\mathscr{V}_{n}: n \in \omega\right\rangle$ be the sequence of open covers defined by

$$
\mathscr{V}_{n}=\mathscr{U}_{0} \wedge \cdots \wedge \mathscr{U}_{n}
$$

Since $\mathrm{S}_{\mathrm{f}}(\mathscr{O}, \mathscr{O}) \bmod$ fin holds, there is a sequence $\left\langle F_{n}: n \in \omega\right\rangle$ and a finite $N \subset \omega$ such that

a. $F_{n} \subset \mathscr{V}_{n}$ is finite and therefore, for each $V \in F_{n}, V=U_{0}^{V} \cap \cdots \cap U_{n}^{V}$ with $U_{i}^{V} \in \mathscr{U}_{i}$;

b. $\bigcup_{n \in \omega} F_{n} \in \mathscr{O}$;

c. $\left\{k \in \omega:\left|F_{k}\right|>f(k)\right\}=N$.

Let $n_{\max }=\max N$ and $G=\bigcup_{n \leq n_{\max }} F_{n}$. For each $V \in G$ there is a $k_{V} \in \omega$ such that $V=U_{0}^{V} \cap$ $\cdots \cap U_{k_{V}}^{V}$, so let $U_{V}=U_{0}^{V}$ and $G_{0}=\left\{U_{V}: V \in G\right\}$. For $0<n \leq n_{\max }$, let $G_{n}=\left\{U_{n}\right\}$ for any $U_{n} \in \mathscr{U}_{n}$. For $n>n_{\max }$, let $G_{n}=\left\{U_{n}^{V}: V \in F_{n}\right\}$. Then

1. $G_{0}$ is finite;

2. $\left|G_{n}\right|=1$, if $0<n \leq n_{\max }$;

3. $\left|G_{n}\right| \leq\left|F_{n}\right|$, if $n_{\max } \leq n$.

therefore,

a. $G_{n} \subset \mathscr{U}_{n}$ for every $n \in \omega$;

b. $\bigcup_{n \in \omega} G_{n} \in \mathscr{O}$;

c. $\left\{n \in \omega:\left|G_{n}\right|>f(n)\right\} \subset 1$.

It follows that $\mathrm{S}_{\mathrm{f}}(\mathscr{O}, \mathscr{O}) \bmod 1$ holds.

Proposition 8.3.7. For all $k \in \mathbb{N}$ and $f \in \mathbb{N}^{\omega}$ :

$$
\mathrm{S}_{1}(\mathscr{O}, \mathscr{O}) \bmod 1 \Longleftrightarrow \mathrm{S}_{\mathrm{k}}(\mathscr{O}, \mathscr{O}) \bmod 1 \Longleftrightarrow \mathrm{S}_{\mathrm{f}}(\mathscr{O}, \mathscr{O}) \bmod 1
$$

Proof. Fix a space $X$. The implications

$$
\mathrm{S}_{1}(\mathscr{O}, \mathscr{O}) \bmod 1 \Longrightarrow \mathrm{S}_{\mathrm{k}}(\mathscr{O}, \mathscr{O}) \bmod 1 \Longrightarrow \mathrm{S}_{\mathrm{f}}(\mathscr{O}, \mathscr{O}) \bmod 1
$$

are clear, so suppose $\mathrm{S}_{\mathrm{f}}(\mathscr{O}, \mathscr{O}) \bmod 1$ holds and let $\left\langle\mathscr{U}_{n}: n \in \omega\right\rangle$ be a sequence of open covers of $X$. Then we recursively define a new sequence of open covers $\left\langle\mathscr{W}_{n}: n \in \omega\right\rangle$ as it follows: First, let $\mathscr{W}_{0}=\mathscr{U}_{0}$. Then we let: 
- $\mathscr{W}_{1}=\bigwedge_{i=1}^{i=1+f(1)} \mathscr{U}_{i}$

- $\mathscr{W}_{2}=\bigwedge_{i=2+f(1)}^{i=2+f(1)+f(2)} \mathscr{U}_{i}$;

- $\mathscr{W}_{3}=\bigwedge_{i=3+f(1)+f(2)}^{i=3+f(1)+f(2)+f(3)} \mathscr{U}_{i}$;

- and so on.

If we apply property $\mathrm{S}_{\mathrm{f}}(\mathscr{O}, \mathscr{O}) \bmod 1$ to $\left\langle\mathscr{W}_{n}: n \in \omega\right\rangle$, then we clearly can find a sequence $\left\langle\mathscr{V}_{n}: n \in \omega\right\rangle$ such that $\bigcup_{n \in \omega} \mathscr{V}_{n} \in \mathscr{O}, \mathscr{V}_{0} \subset \mathscr{U}_{0}$ is finite and, for each $n>0, \mathscr{V}_{n} \subset \mathscr{U}_{n}$ and $\left|\mathscr{V}_{n}\right| \leq 1$. Therefore, $\mathrm{S}_{1}(\mathscr{O}, \mathscr{O}) \bmod 1$ holds.

About the covering games, we note that Proposition 8.2.11 still holds if we replace " $\mathrm{G}_{\text {bnd }}(\mathscr{O}, \mathscr{O})$ " by " $\mathrm{G}_{1}(\mathscr{O}, \mathscr{O}) \bmod$ fin" or " $\mathrm{G}_{1}(\mathscr{O}, \mathscr{O}) \bmod 1$ ". Again, this is no coincidence. But before looking further into this matter, consider the following lemma.

Lemma 8.3.8. Let $X$ be a space. Then for every $\mathscr{U}_{0} \in \mathscr{O}$, ALICE has a winning strategy $\gamma$ in $\mathrm{G}_{1}(\mathscr{O}, \mathscr{O}) \bmod 1$ such that $\gamma(\langle\rangle)=\mathscr{U}_{0}$ if, and only if, for every $k \in \mathbb{N}$ there is a winning strategy $\gamma_{k}$ for ALICE in $\mathrm{G}_{\mathrm{k}}(\mathscr{O}, \mathscr{O}) \bmod 1$ with $\gamma_{k}(\langle\rangle)=\mathscr{U}_{0}$.

Proof. Let $\mathscr{U}_{0} \in \mathscr{O}$, suppose there is a winning strategy $\gamma$ for ALICE in $G_{1}(\mathscr{O}, \mathscr{O}) \bmod 1$ such

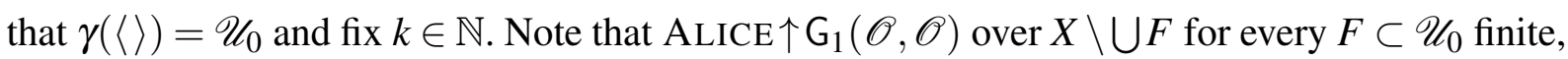
which implies (by Theorem 4.1.37) that there is a winning strategy $\gamma_{k}^{F}$ for $\operatorname{ALICE}$ in $\mathrm{G}_{\mathrm{k}}(\mathscr{O}, \mathscr{O})$ over $X \backslash \bigcup F$. Now, consider the following strategy:

- First, let $\gamma_{k}(\langle\rangle)=\mathscr{U}_{0}$;

- If Bов then chooses $F_{0} \subset \gamma_{k}(\langle\rangle)$ finite, let

$$
\gamma_{k}\left(\left\langle F_{0}\right\rangle\right)=\left\{\mathrm{V} \text { open }: V \cap\left(X \backslash \bigcup F_{0}\right) \in \gamma_{k}^{F_{0}}(\langle\rangle)\right\} \cup\left\{\bigcup F_{0}\right\}
$$

- If BoB then chooses $F_{1}=\left\{V_{1}\right\} \subset \gamma_{k}\left(\left\langle F_{0}\right\rangle\right)$, let

$$
\gamma_{k}\left(\left\langle F_{0}, \mathscr{V}_{1}\right\rangle\right)=\left\{\mathrm{V} \text { open }: V \cap\left(X \backslash \bigcup F_{0}\right) \in \gamma_{k}^{F_{0}}\left(\left\langle F_{1}\right\rangle\right)\right\} \cup\left\{\bigcup F_{0}\right\}
$$

(we are assuming here that Вов will not choose $V_{1}=\bigcup F_{0}$, since its points were already covered in the first inning);

- And so on.

Clearly, $\gamma_{k}$ has the desired properties.

The other implication is obvious.

Now, the following theorem will help us show one of the main results of this paper. 
Theorem 8.3.9. Let $X$ be a regular space. Then $\mathrm{BOB} \uparrow \mathrm{G}_{1}(\mathscr{O}, \mathscr{O}) \bmod 1$ if, and only if, there is a compact set $K \subset X$ such that, for every open set $V \supset K, \mathrm{BoB} \uparrow \mathrm{G}_{1}(\mathscr{O}, \mathscr{O})$ over $X \backslash V$.

Proof. Suppose there is a compact set $K \subset X$ such that, for every open set $V \supset K$, there is a winning strategy $\sigma_{1}^{V}$ for Вов in $\mathrm{G}_{1}(\mathscr{O}, \mathscr{O})$ over $X \backslash V$. Then we define the following strategy $\sigma$ for $\mathrm{B}$ OB in $\mathrm{G}_{1}(\mathscr{O}, \mathscr{O}) \bmod 1$ :

- If ALICE starts with $\mathscr{U}_{0} \in \mathscr{O}$, let $\sigma\left(\mathscr{U}_{0}\right)$ be a finite subcover for $K$ and let $V=\bigcup \sigma\left(\mathscr{U}_{0}\right)$.

- After that, if $\left\langle\mathscr{U}_{0}, \ldots, \mathscr{U}_{n}\right\rangle$ is played by ALICE, let $\sigma\left(\left\langle\mathscr{U}_{0}, \ldots, \mathscr{U}_{n}\right\rangle\right)=\sigma_{1}^{V}\left(\left\langle\mathscr{U}_{1}, \ldots, \mathscr{U}_{n}\right\rangle\right)$.

Then, clearly, $\sigma$ is a winning strategy.

Now, suppose $\sigma$ is a winning strategy for BоB in $\mathrm{G}_{1}(\mathscr{O}, \mathscr{O}) \bmod 1$.

Claim 8.3.10. The set

$$
K=\bigcap_{\mathscr{U} \in \mathscr{O}} \overline{\bigcup \sigma(\langle\mathscr{U}\rangle)}
$$

is compact.

Proof. Indeed, let $\mathscr{C}$ be an open cover for $K$ and for each $x \in K$, let $U_{x} \in \mathscr{C}$ be such that $x \in U_{x}$. Since $X$ is regular, for every $x \in K$ there is an open set $V_{x}$ such that $x \in V_{x} \subset \overline{V_{x}} \subset U_{x}$. On the other hand, for each $x \in X \backslash K$ we consider an open set $V_{x}$ such that $x \in V_{x}$ and $\overline{V_{x}} \cap K=\emptyset$ (because $K$ is closed and $X$ is regular). Now, let $\mathscr{U}=\left\{V_{x}: x \in X\right\} \in \mathscr{O}$. In this case, note that

$$
K \subset \overline{\bigcup \sigma(\langle\mathscr{U}\rangle)} \text {. }
$$

Consider $\mathscr{A}=\left\{V_{x}:(x \in K) \wedge\left(V_{x} \in \sigma(\langle\mathscr{U}\rangle)\right)\right\}=\left\{V_{x_{1}}, \ldots, V_{x_{n}}\right\}$, with $x_{1}, \ldots, x_{n} \in K$. Then $K \subset$ $\bar{\cup} \mathscr{A}$. Finally, note that $\left\{U_{x_{1}}, \ldots, U_{x_{n}}\right\} \subset \mathscr{C}$ is a finite subcover of $K$.

Now, let $V$ be an open set containing $K$. Note that since $\mathrm{BoB} \uparrow \mathrm{G}_{1}(\mathscr{O}, \mathscr{O}) \bmod 1, X$ is Lindelöf, and since $X \backslash V$ is closed, $X \backslash V$ is Lindelöf. With that in mind, if we consider the open cover $\{X \backslash \overline{\cup \sigma(\langle\mathscr{U}\rangle)}: \mathscr{U} \in \mathscr{O}\}$ of $X \backslash V$, we may obtain a countable subcover $\left\{X \backslash \overline{\cup \sigma\left(\left\langle\mathscr{U}_{n}\right\rangle\right)}: n \in \mathbb{N}\right\}$. If $\mathscr{V}$ is an open cover of $X \backslash V$, let $\mathscr{V}^{\prime}=\mathscr{V} \cup\{V\} \in \mathscr{O}$ and fix an enumeration $\left\{p_{n}: n \in \mathbb{N}\right\}$ of the prime numbers of $\omega$. Now we have everything at hand to define a winning strategy $\sigma_{1}^{V}$ for BoB in $\mathrm{G}_{1}(\mathscr{O}, \mathscr{O})$ over $X \backslash V$ :

$$
\sigma_{1}^{V}\left(\left\langle\mathscr{V}_{0}, \ldots, \mathscr{V}_{n}\right\rangle\right)=\left\{\begin{array}{l}
\sigma\left(\left\langle\mathscr{U}_{k}, \mathscr{V}_{p_{k}^{1}}^{\prime}, \ldots, \mathscr{V}_{p_{k}^{m}}^{\prime}\right\rangle\right) \backslash\{V\}, \text { if } n=p_{k}^{m} \text { for some } k, m \in \mathbb{N} \\
\left\{U_{n}\right\} \text { with } U_{n} \in \mathscr{V}_{n} \text { (anyone!), otherwise. }
\end{array}\right.
$$

To show that $\sigma_{1}^{V}$ is, indeed, winning, let $y \in X \backslash V$ and consider $\left\langle\mathscr{V}_{n}: n \in \omega\right\rangle$ as any play from ALICE in $\mathrm{G}_{1}(\mathscr{O}, \mathscr{O})$ over $X \backslash V$. Since $\left\{X \backslash \overline{\cup \sigma\left(\left\langle\mathscr{U}_{n}\right\rangle\right)}: n \in \mathbb{N}\right\}$ covers $X \backslash V, y \notin \overline{\cup \sigma\left(\left\langle\mathscr{U}_{k}\right\rangle\right)}$ for some $k \in \mathbb{N}$. But since $\sigma$ is a winning strategy in $\mathrm{G}_{1}(\mathscr{O}, \mathscr{O}) \bmod 1, y$ must be covered by some of $\sigma$ 's responses to ALICE's play $\left\langle\mathscr{U}_{k}\right\rangle^{\wedge}\left\langle\mathscr{V}_{p_{k}^{n}}^{\prime}: n \in \mathbb{N}\right\rangle$, so $\sigma_{1}^{V}$ covers $y$ and, therefore, is a winning strategy. 
But how does this new selection principles relate to the "bounded versions" presented here? As it turns out, in a very simple way.

Theorem 8.3.11. $\mathrm{S}_{\mathrm{bnd}}(\mathscr{O}, \mathscr{O})$ holds if, and only if, $\mathrm{S}_{1}(\mathscr{O}, \mathscr{O}) \bmod 1$ holds.

Proof. The implication

$$
\mathrm{S}_{1}(\mathscr{O}, \mathscr{O}) \bmod 1 \Longrightarrow \mathrm{S}_{\text {bnd }}(\mathscr{O}, \mathscr{O})
$$

is clear, so suppose $\mathrm{S}_{\text {bnd }}(\mathscr{O}, \mathscr{O})$ holds. We define $f \in \mathbb{N}^{\omega}$ as $f(n)=n+1$. Now, since for every $k \in \omega$ the set $\{n \in \omega: k>f(n)\}$ is finite, the result follows from the fact that $\mathrm{S}_{\text {bnd }}(\mathscr{O}, \mathscr{O})$ holds if, and only if, $\mathrm{S}_{\mathrm{f}}(\mathscr{O}, \mathscr{O}) \bmod$ fin holds and by Propositions 8.3.6 and 8.3.7.

Regarding the games, $\mathrm{G}_{\mathrm{bnd}}(\mathscr{O}, \mathscr{O})$ is equivalent (over Hausdorff spaces) to $\mathrm{G}_{1}(\mathscr{O}, \mathscr{O}) \bmod 1$. We show this assertion in the following theorems.

Theorem 8.3.12. Alice has a winning strategy in $\mathrm{G}_{\mathrm{bnd}}(\mathscr{O}, \mathscr{O})$ if, and only if, AliCE has a winning strategy in $\mathrm{G}_{1}(\mathscr{O}, \mathscr{O}) \bmod 1$.

The idea behind the proof of Theorem 8.3.12 is similar to the one presented in the proof of Theorem 8.2.5.

The main difference here is that ALICE cannot just pretend the game restarted at any inning without losing important information, because В ОВ have indeed covered a portion of the space thus far. Lemma 8.3.8, however, gives us instructions of how she can switch between strategies pretending the game is back to the second inning without losing this important information.

Formally speaking:

Proof of Theorem 8.3.12. The implication

$$
\operatorname{ALICE} \uparrow G_{\text {bnd }}(\mathscr{O}, \mathscr{O}) \Longrightarrow \operatorname{ALICE} \uparrow G_{1}(\mathscr{O}, \mathscr{O}) \bmod 1
$$

is clear.

So, suppose $\gamma$ is a winning strategy for ALICE in $\mathrm{G}_{1}(\mathscr{O}, \mathscr{O}) \bmod 1$ and let $\gamma_{k}$, for each $k \in \mathbb{N}$ be as in Lemma 8.3.8 with $\mathscr{U}_{0}=\gamma(\langle\rangle)$ (that is, such that $\gamma_{k}(\langle\rangle)=\mathscr{U}_{0}$ for every $k \in \mathbb{N}$ ). We will assume that $\gamma$ and $\gamma_{k}$, for every $k \in \mathbb{N}$, tell ALICE to play refinements of $\mathscr{U}_{0}$ in every turn. Now consider the following strategy:

- First, let $\tilde{\gamma}(\langle\rangle)=\mathscr{U}_{0}$.

- If Bов chooses $F_{0} \subset \mathscr{U}_{0}$ with $\left|F_{0}\right|=k_{0}$ for some $k_{0} \in \mathbb{N}$, let

$$
\tilde{\gamma}\left(\left\langle F_{0}\right\rangle\right)=\gamma_{k_{0}}\left(\left\langle F_{0}\right\rangle\right)
$$


- If BoB chooses $F_{1} \subset \tilde{\gamma}\left(\left\langle F_{0}\right\rangle\right)$ such that $\left|F_{1}\right| \leq k_{0}$, then let

$$
\tilde{\gamma}\left(\left\langle F_{0}, F_{1}\right\rangle\right)=\gamma_{k_{0}}\left(\left\langle F_{0}, F_{1}\right\rangle\right)
$$

otherwise, if $\left|F_{1}\right|=k_{1}>k_{0}$, then for each $V \in F_{1}$ fix $U_{V} \in \mathscr{U}_{0}$ such that $V \subset U_{V}$ and let

$$
\tilde{\gamma}\left(\left\langle F_{0}, F_{1}\right\rangle\right)=\gamma_{k_{1}}\left(\left\langle F_{1}^{\prime}\right\rangle\right)
$$

with $F_{1}^{\prime}=\left\{U_{V}: V \in F_{1}\right\} \cup F_{0}$;

- And so on.

Clearly, $\tilde{\gamma}$ is a winning strategy for ALICE in $\mathrm{G}_{\mathrm{bnd}}(\mathscr{O}, \mathscr{O})$.

Theorem 8.3.13. Let $X$ be a Hausdorff space. Then $\mathrm{BoB} \uparrow \mathrm{G}_{\mathrm{bnd}}(\mathscr{O}, \mathscr{O})$ if, and only if, $\mathrm{BoB} \uparrow \mathrm{G}_{1}(\mathscr{O}, \mathscr{O}) \bmod 1$.

Proof. The implication

$$
\mathrm{BoB} \uparrow \mathrm{G}_{1}(\mathscr{O}, \mathscr{O}) \bmod 1 \Longrightarrow \mathrm{BoB} \uparrow \mathrm{G}_{\mathrm{bnd}}(\mathscr{O}, \mathscr{O})
$$

is clear, so let $\sigma$ be a winning strategy for BoB in $\mathrm{G}_{\mathrm{bnd}}(\mathscr{O}, \mathscr{O})$.

Note that we can assume that ALICE plays always with refinements of her first cover played in the game. For each $\mathscr{U} \in \mathscr{O}$, let $s_{\mathscr{U}} \in{ }^{<\omega} \mathscr{O}$ and $m_{\mathscr{U}} \in \mathbb{N}$ be as in Lemma 8.2.4 for $r=\langle\mathscr{U}\rangle$. Now, fixed $\mathscr{U} \in \mathscr{O}$, we fix, for each $U \in \bigcup_{k \in \operatorname{dom}\left(s_{\mathscr{U}}\right)+1} \sigma\left(s_{\mathscr{U}} \uparrow k\right), V_{U} \in \mathscr{U}$ such that $U \subset V_{U}$. Then we let

$$
\tilde{\sigma}(\langle\mathscr{U}\rangle)=\left\{V_{U}: U \in \bigcup_{k \in \operatorname{dom}\left(s_{\mathscr{U}}\right)+1} \sigma\left(s_{\mathscr{U}} \uparrow k\right)\right\} .
$$

Note that, by our hypothesis, Вов $\uparrow \mathrm{G}_{\mathrm{m}_{\mathscr{U}}}(\mathscr{O}, \mathscr{O})$ over $X \backslash \cup \tilde{\sigma}(\langle\mathscr{U}\rangle)$ for each $\mathscr{U} \in \mathscr{O}$, so it follows from Theorem 4.1.37 that there is a winning strategy $\sigma_{\mathscr{U}}$ for BOB in $\mathrm{G}_{1}(\mathscr{O}, \mathscr{O})$ over $X \backslash \bigcup \tilde{\sigma}(\langle\mathscr{U}\rangle)$ for each $\mathscr{U} \in \mathscr{O}$. Then we define, for each $s \in{ }^{<\omega} \mathscr{O}$,

$$
\tilde{\sigma}(\langle\mathscr{U}\rangle\urcorner s)=\sigma_{\mathscr{U}}(s)
$$

and it is clear that the strategy $\tilde{\sigma}$ we have just defined is a winning strategy for BoB in $\mathrm{G}_{1}(\mathscr{O}, \mathscr{O}) \bmod 1$.

Corollary 8.3.14. Let $X$ be Hausdorff space. Then, for every $f \in \mathbb{N}^{\omega}$,

$$
\mathrm{BOB} \uparrow \mathrm{G}_{\mathrm{bnd}}(\mathscr{O}, \mathscr{O}) \Longleftrightarrow \mathrm{BOB} \uparrow \mathrm{G}_{\mathrm{f}}(\mathscr{O}, \mathscr{O}) \bmod \text { fin } \Longleftrightarrow \mathrm{BoB} \uparrow \mathrm{G}_{\mathrm{f}}(\mathscr{O}, \mathscr{O}) \bmod 1
$$

Corollary 8.3.15. The games $\mathrm{G}_{\mathrm{bnd}}(\mathscr{O}, \mathscr{O})$ and $\mathrm{G}_{1}(\mathscr{O}, \mathscr{O}) \bmod 1$ are equivalent over every Hausdorff space.

And finally: 
Theorem 8.3.16. Let $X$ be a regular space. Then $\mathrm{BoB} \uparrow \mathrm{G}_{\mathrm{bnd}}(\mathscr{O}, \mathscr{O})$ if, and only if, there is a compact set $K \subset X$ such that, for every open set $V \supset K$, BоB $\uparrow G_{1}(\mathscr{O}, \mathscr{O})$ on $X \backslash V$.

Proof. It follows directly from Theorems 8.3.9 and 8.3.13.

Theorem 8.3.16 is useful to characterize even stricter sets on metric spaces:

Corollary 8.3.17. Let $X$ be a regular space such that every compact subset is a $G_{\delta}$ subset (e.g. a metrizable space). Then $\mathrm{BOB} \uparrow \mathrm{G}_{\mathrm{bnd}}(\mathscr{O}, \mathscr{O})$ if, and only if, there is a compact set $K \subset X$ and a countable set $N \subset X$ such that $X=K \cup N$.

Proof. This a direct implication of Theorem 8.3.16 combined with 4.1.4.

We end this section with a simple remark: one may wonder whether changing the definition of the "mod 1 " variation of the games (or selection principles) to, instead of allowing the choices to exceed the binding function in the first inning (or element of the sequence, in the case of selection principles), allowing the choices to exceed the binding function in the innings of a fixed finite $F \subset \omega$ (which would give rise to some "mod $F$ " variation) would be any different from "mod 1". We note that, in view of Corollary 8.3.15 (and Theorem 8.3.11, in the case of the selection principle), everything collapses to the "mod 1 " variation in the covering case.

\subsection{The analogous to Pawlikowski's and Hurewicz's re- sults}

As it turns out, our previous results can help us show an analogous theorem here, in the "bounded" variation. The following proof is heavily inspired by the simplified proof of Theorem 4.1.32 that can be seen, for instance, in [Szewczak and Tsaban 2019].

Theorem 8.4.1. $\mathrm{S}_{\mathrm{bnd}}(\mathscr{O}, \mathscr{O}) \Longleftrightarrow \operatorname{ALICE} \Varangle \mathrm{G}_{\mathrm{bnd}}(\mathscr{O}, \mathscr{O})$

Proof. Implication ALICE $\Varangle \mathrm{G}_{\text {bnd }}(\mathscr{O}, \mathscr{O}) \Longrightarrow \mathrm{S}_{\text {bnd }}(\mathscr{O}, \mathscr{O})$ is clear by Proposition 8.2.3.

To show the reverse implication, by Proposition 8.3.11 and Theorem 8.3.12, it suffices to show that

$$
\mathrm{S}_{1}(\mathscr{O}, \mathscr{O}) \bmod 1 \Longrightarrow \operatorname{ALICE} \nsucc \mathrm{G}_{1}(\mathscr{O}, \mathscr{O}) \bmod f i n,
$$

so suppose $S_{1}(\mathscr{O}, \mathscr{O})$ mod 1 holds and let $\gamma$ be a strategy for AlicE in $\mathrm{G}_{1}(\mathscr{O}, \mathscr{O})$ mod fin. For simplicity's sake, in the rest of this proof we will write " $\{V\}$ " simply as " $V$ ".

We then recursively define the following strategy $\tilde{\gamma}$ for ALICE in $\mathrm{G}_{\text {fin }}(\mathscr{O}, \mathscr{O})$ and function $f:$

We first let $\tilde{\gamma}(\langle\rangle)=\gamma(\langle\rangle)$. Then, for each $V_{0} \in \tilde{\gamma}(\langle\rangle)$, 


$$
f\left(\left\langle V_{0}\right\rangle\right)=V_{0},
$$

and, for each finite $\mathscr{F}_{0} \subset \tilde{\gamma}(\langle\rangle)$, let

$$
f\left(\left\langle\mathscr{F}_{0}\right\rangle\right)=\left\{f\left(\left\langle V_{0}\right\rangle\right): V_{0} \in \mathscr{F}_{0}\right\}=\mathscr{F}_{0} .
$$

Suppose $\mathscr{F}_{0}$ was chosen by Вов. Then we let

$$
\tilde{\gamma}\left(\left\langle\mathscr{F}_{0}\right\rangle\right)=\gamma\left(\left\langle f\left(\left\langle\mathscr{F}_{0}\right\rangle\right)\right\rangle\right) \wedge \bigwedge_{V_{0} \in \mathscr{F}_{0}} \gamma\left(\left\langle f\left(\left\langle V_{0}\right\rangle\right)\right\rangle\right) .
$$

Now, for each $V_{0} \in \mathscr{F}_{0}$ and $V_{1} \in \tilde{\gamma}\left(\left\langle\mathscr{F}_{0}\right\rangle\right)$, define

$$
\begin{gathered}
f\left(\left\langle V_{0}, V_{1}\right\rangle\right)=V \in \gamma\left(\left\langle f\left(\left\langle V_{0}\right\rangle\right)\right\rangle\right) \text { such that } V \supset V_{1} ; \\
f\left(\left\langle\mathscr{F}_{0}, V_{1}\right\rangle\right)=V \in \gamma\left(\left\langle f\left(\left\langle\mathscr{F}_{0}\right\rangle\right)\right\rangle\right) \text { such that } V \supset V_{1} ;
\end{gathered}
$$

and, for each finite $\mathscr{F}_{1} \subset \tilde{\gamma}\left(\left\langle\mathscr{F}_{0}\right\rangle\right)$,

$$
f\left(\left\langle\mathscr{F}_{0}, \mathscr{F}_{1}\right\rangle\right)=\left\{f\left(\left\langle\mathscr{F}_{0}, V_{1}\right\rangle\right): V_{1} \in \mathscr{F}_{1}\right\} .
$$

Suppose $\mathscr{F}_{1}$ is then chosen by Вов. Then we let

$$
\begin{aligned}
\tilde{\gamma}\left(\left\langle\mathscr{F}_{0}, \mathscr{F}_{1}\right\rangle\right) & =\gamma\left(\left\langle f\left(\left\langle\mathscr{F}_{0}\right\rangle\right), f\left(\left\langle\mathscr{F}_{0}, \mathscr{F}_{1}\right\rangle\right)\right\rangle\right) \wedge\left(\bigwedge_{V_{1} \in \mathscr{F}_{1}} \gamma\left(\left\langle f\left(\left\langle\mathscr{F}_{0}\right\rangle\right), f\left(\left\langle, \mathscr{F}_{0}, V_{1}\right\rangle\right)\right\rangle\right)\right) \wedge \\
& \wedge\left(\bigwedge_{V_{0} \in \mathscr{F}_{0} V_{1} \in \mathscr{F}_{1}} \gamma\left(\left\langle f\left(\left\langle V_{0}\right\rangle\right), f\left(\left\langle V_{0}, V_{1}\right\rangle\right)\right\rangle\right)\right),
\end{aligned}
$$

for each $V_{0} \in \mathscr{F}_{0}, V_{1} \in \mathscr{F}_{1}$ and $V_{2} \in \tilde{\gamma}\left(\left\langle\mathscr{F}_{0}, \mathscr{F}_{1}\right\rangle\right)$,

$$
\begin{aligned}
f\left(\left\langle V_{0}, V_{1}, V_{2}\right\rangle\right) & =V \in \gamma\left(\left\langle f\left(\left\langle V_{0}\right\rangle\right), f\left(\left\langle V_{0}, V_{1}\right\rangle\right)\right\rangle\right) \text { such that } V \supset V_{2} ; \\
f\left(\left\langle\mathscr{F}_{0}, V_{1}, V_{2}\right\rangle\right) & =V \in \gamma\left(\left\langle f\left(\left\langle\mathscr{F}_{0}\right\rangle\right), f\left(\left\langle\mathscr{F}_{0}, V_{1}\right\rangle\right)\right\rangle\right) \text { such that } V \supset V_{2} ; \\
f\left(\left\langle\mathscr{F}_{0}, \mathscr{F}_{1}, V_{2}\right\rangle\right) & =V \in \gamma\left(\left\langle f\left(\left\langle\mathscr{F}_{0}\right\rangle\right), f\left(\left\langle\mathscr{F}_{0}, \mathscr{F}_{1}\right\rangle\right)\right\rangle\right) \text { such that } V \supset V_{2},
\end{aligned}
$$

and, for each finite $\mathscr{F}_{2} \subset \tilde{\gamma}\left(\left\langle\mathscr{F}_{0}, \mathscr{F}_{1}\right\rangle\right)$,

$$
f\left(\left\langle\mathscr{F}_{0}, \mathscr{F}_{1}, \mathscr{F}_{2}\right\rangle\right)=\left\{f\left(\left\langle\mathscr{F}_{0}, \mathscr{F}_{1}, V_{2}\right\rangle\right): V_{2} \in \mathscr{F}_{2}\right\}
$$

Now let us look at the general case. Suppose we have defined $\tilde{\gamma}$ and $f$ up to $s \in \operatorname{dom} \tilde{\gamma}$ in such a way that, for every $k \leq|s|$ : 


$$
\begin{aligned}
\tilde{\gamma}(s\lceil k)= & \gamma(\langle f(s\lceil 1), \ldots, f(s\lceil k)\rangle) \wedge \\
& \wedge\left(\bigwedge _ { V _ { k - 1 } \in s ( k - 1 ) } \gamma \left(\left\langle f(s\lceil 1)), \ldots, f\left(s\lceil k-1), f\left(\left(s\lceil k-1)^{\wedge} V_{k-1}\right)\right\rangle\right)\right) \wedge\right.\right. \\
& \vdots \\
& \wedge\left(\bigwedge_{V_{0} \in s(0)} \bigwedge_{V_{1} \in s(1)} \cdots \bigwedge_{V_{k-1} \in s(k-1)} \gamma\left(\left\langle f\left(\left\langle V_{0}\right\rangle\right), \ldots, f\left(\left\langle V_{0}, \ldots, V_{k-1}\right\rangle\right)\right\rangle\right)\right),
\end{aligned}
$$

for all $V_{0} \in s(0), \ldots, V_{k-1} \in s(k-1), V_{k} \in \tilde{\gamma}(s\lceil k)$ :

$$
\begin{aligned}
f\left(\left\langle V_{0}, \ldots, V_{k-1}, V_{k}\right\rangle\right)= & V \in \gamma\left(\left\langle f\left(\left\langle V_{0}\right\rangle\right), \ldots, f\left(\left\langle V_{0}, \ldots, V_{k-1}\right\rangle\right)\right\rangle\right) \\
& \text { such that } V \supset V_{k} ; \\
f\left(\left\langle s(0), V_{1}, \ldots, V_{k-1}, V_{k}\right\rangle\right)= & \left.V \in \gamma\left\langle f(\langle s(0)\rangle), f\left(\left\langle s(0), V_{1}\right\rangle\right), \ldots, f\left(\left\langle s(0), \ldots, V_{k-1}\right\rangle\right)\right\rangle\right) \\
& \text { such that } V \supset V_{k} ; \\
& \vdots \\
f\left(\left(s\lceil k)^{\frown} V_{k}\right)=\right. & V \in \gamma\left(\langle f(s \uparrow 1), \ldots, f(s\lceil k)\rangle) \text { such that } V \supset V_{k},\right.
\end{aligned}
$$

and for every $\mathscr{F}_{k} \subset \tilde{\gamma}(s\lceil k)$,

$$
f\left(\left(s\lceil k)^{\frown} \mathscr{F}_{k}\right)=\left\{f\left(\left(s\lceil k)^{\frown} V_{k}\right): V_{k} \in \mathscr{F}_{k}\right\} .\right.\right.
$$

Then if BoB chooses $\mathscr{F}_{n} \subset \tilde{\gamma}(s)$ we let

$$
\begin{aligned}
\tilde{\gamma}\left(s^{\frown \mathscr{F}_{n}}\right)= & \gamma\left(\left\langle f(s \uparrow 1), \ldots, f(s), f\left(s^{\frown \mathscr{F}_{n}}\right)\right\rangle\right) \wedge \\
& \wedge\left(\bigwedge_{V_{n} \in \mathscr{F}_{n}} \gamma\left(\langle f(s \uparrow 1)), \ldots, f(s), f\left(s^{\frown} V_{n}\right)\right\rangle\right) \wedge \\
& \vdots \\
& \wedge\left(\bigwedge_{V_{0} \in s(0)} \cdots \bigwedge_{V_{n-1} \in s(n-1)} \bigwedge_{V_{n} \in \mathscr{F}_{n}} \gamma\left(\left\langle f\left(\left\langle V_{0}\right\rangle\right), \ldots, f\left(\left\langle V_{0}, \ldots, V_{n}\right\rangle\right)\right\rangle\right)\right),
\end{aligned}
$$

for all $V_{0} \in s(0), \ldots, V_{n-1} \in s(n-1), V_{n} \in \mathscr{F}_{n}, V_{n+1} \in \tilde{\gamma}\left(s^{\frown} \mathscr{F}_{n}\right)$ : 


$$
\begin{aligned}
f\left(\left\langle V_{0}, \ldots, V_{n}, V_{n+1}\right\rangle\right)= & V \in \gamma\left(\left\langle f\left(\left\langle V_{0}\right\rangle\right), \ldots, f\left(\left\langle V_{0}, \ldots, V_{n}\right\rangle\right)\right\rangle\right) \\
& \text { such that } V \supset V_{n+1} ; \\
f\left(\left\langle s(0), V_{1}, \ldots, V_{n}, V_{n+1}\right\rangle\right)= & \left.V \in \gamma\left\langle f(\langle s(0)\rangle), f\left(\left\langle s(0), V_{1}\right\rangle\right), \ldots, f\left(\left\langle s(0), \ldots, V_{n}\right\rangle\right)\right\rangle\right) \\
& \text { such that } V \supset V_{n+1} ; \\
& \vdots \\
f\left(s^{\frown} \mathscr{F}_{n}^{\urcorner} V_{n+1}\right)= & V \in \gamma(\langle f(s \nmid 1), \ldots, f(s)\rangle) \text { such that } V \supset V_{n+1},
\end{aligned}
$$

and for every $\mathscr{F}_{n+1} \subset \tilde{\gamma}\left(s^{\frown} \mathscr{F}_{n}\right)$,

$$
f\left(s^{\frown \mathscr{F}_{n}} \mathscr{F}_{n+1}\right)=\left\{f\left(s^{\frown \mathscr{F}_{n}} V_{n+1}\right): V_{n+1} \in \mathscr{F}_{n+1}\right\}
$$

so the recursion is complete.

Now, since $S_{1}(\mathscr{O}, \mathscr{O}) \bmod 1$ holds, $S_{\text {fin }}(\mathscr{O}, \mathscr{O})$ holds and, by Theorem 4.1.12, $\tilde{\gamma}$ is not a winning strategy. Moreover, Вов can play a sequence $\left\langle\mathscr{F}_{n}: n \in \omega\right\rangle$ against $\tilde{\gamma}$ such that $\bigcup_{n \geq m} \cup \mathscr{F}_{n}=X$ for every $m \in \omega$ (to see this, just note that if $\operatorname{ALICE}_{X} \mathrm{G}_{\text {fin }}(\mathscr{O}, \mathscr{O})$ over $X$, then $\operatorname{ALICE} \Varangle \mathrm{G}_{\text {fin }}(\mathscr{O}, \mathscr{O})$ over $\left.X \times \omega\right)$.

CLAIM 8.4.2. There is an $N \in \omega$ and a choice of $V_{n} \in \mathscr{F}_{n}$ for each $n \geq N$ such that $\left(\bigcup_{n \leq N} \mathscr{F}_{n}\right) \cup$ $\left(\bigcup_{n>N} V_{n}\right)=X$.

Proof. For each $n \in \omega$ let

$$
\mathscr{W}_{n}=\left\{V^{k_{0}} \cap \cdots \cap V^{k_{n}}: V^{k_{i}} \in \mathscr{F}_{k_{i}} \text { for all } i \leq n \text { and } k_{0}<k_{1}<\cdots<k_{n}\right\} .
$$

Note that $\mathscr{W}_{n}$ is an open cover for every $n \in \omega$. Then, since $S_{1}(\mathscr{O}, \mathscr{O}) \bmod 1$ holds, we can find $\left\{V^{k_{0}}, \ldots V^{k_{m}}\right\} \subset \mathscr{W}_{0}$ with $V^{k_{i}} \in \mathscr{F}_{k_{i}}$ for each $i \leq k_{m}$ and a single $U_{n} \in \mathscr{W}_{n}$ for each $n>0$ such that $\left(\bigcup_{i \leq m} V^{k_{i}}\right) \cup\left(\bigcup_{n>0} U_{n}\right)=X$.

Let $N=\max \left\{k_{i}: i \leq m\right\}$. Now from each $U_{n}$ we can pick a $V_{l_{n}} \in \mathscr{F}_{l_{n}}$ such that $U_{n} \subset V_{l_{n}}$ and $l_{n} \neq l_{i}$ for all $i<n$. Then if we pick any $V_{k} \in \mathscr{F}_{k}$ when $k \neq l_{n}$ for every $n>0$, the proof is complete.

Now we define a winning play for BОВ against $\gamma$ as it follows. For each inning $n \leq N$, let BоB respond to $\gamma$ with $f\left(\left\langle\mathscr{F}_{i}: i \leq n\right\rangle\right)$. Then, for each $n \geq N$, let BoB respond to $\gamma$ with $f\left(\left\langle\mathscr{F}_{i}: i \leq N\right\rangle^{\wedge}\left\langle V_{j}: j \leq n\right\rangle\right)$. It follows from the definition of $f$ and from Claim 8.4.2 that Вов wins this play in $\mathrm{G}_{1}(\mathscr{O}, \mathscr{O})$ modfin, hence $\gamma$ is not a winning strategy.

One may wonder if Theorem 8.3.16 still holds if we replace "BoB $\uparrow G_{\text {bnd }}(\mathscr{O}, \mathscr{O})$ " by "S $\mathrm{S}_{\text {bnd }}(\mathscr{O}, \mathscr{O})$ " and "Bов $\uparrow \mathrm{G}_{1}(\mathscr{O}, \mathscr{O})$ " by " $\mathrm{S}_{1}(\mathscr{O}, \mathscr{O})$ ". The answer is yes. But to show that, let us first take another step back and define yet another variation of the classical selection principles. 
Definition 8.4.3. Let $(X, \tau)$ be a topological space. We say the property $\mathrm{S}_{1}^{\mathrm{s}}(\mathscr{O}, \mathscr{O}) \bmod 1$ holds if for every open cover $\mathscr{U}$ there is a $\mathscr{V} \subset \mathscr{U}$ finite such that $S_{1}(\mathscr{O}, \mathscr{O})$ holds on $X \backslash \bigcup \mathscr{V}$.

At first glance, this new variation may seem stronger than $S_{1}(\mathscr{O}, \mathscr{O}) \bmod 1$. However, we will show later that they are equivalent selection principles. This will be useful because:

Proposition 8.4.4. Let $X$ be a regular space. Then $\mathrm{S}_{1}^{\mathrm{s}}(\mathscr{O}, \mathscr{O}) \bmod 1$ holds if, and only if, there is a compact set $K \subset X$ such that, for every open set $V \supset K, \mathrm{~S}_{1}(\mathscr{O}, \mathscr{O})$ holds on $X \backslash V$.

Proof. Analogous to the proof of Theorem 8.3.9.

Proposition 8.4.5. $S_{1}^{s}(\mathscr{O}, \mathscr{O}) \bmod 1 \Longleftrightarrow \operatorname{ALICE} \Varangle G_{1}(\mathscr{O}, \mathscr{O}) \bmod 1$

Proof. Suppose $\mathrm{S}_{1}^{\mathrm{s}}(\mathscr{O}, \mathscr{O}) \bmod 1$ holds and let $\gamma$ be a strategy for $\operatorname{AlICE}$ in $\mathrm{G}_{1}(\mathscr{O}, \mathscr{O}) \bmod 1$. Then there is a $\mathscr{V} \subset \gamma(\langle\rangle)$ such that $S_{1}(\mathscr{O}, \mathscr{O})$ holds over $X \backslash \cup \mathscr{V}$, so it follows from Theorem 4.1.32 that $\gamma$ cannot be a winning strategy.

On the other hand, suppose $S_{1}^{s}(\mathscr{O}, \mathscr{O}) \bmod 1$ fails. Then there is an open cover $\mathscr{U}$ such that $\mathrm{S}_{1}(\mathscr{O}, \mathscr{O})$ fails over $X \backslash \cup \mathscr{V}$ for every finite $\mathscr{V} \subset \mathscr{U}$. Let $\gamma(\langle\rangle)=\mathscr{U}$ and, if BoB responds with a finite $\mathscr{V} \subset \mathscr{U}$, then ALICE can simply use the sequence of open covers of $X \backslash \bigcup \mathscr{V}$ that witnesses that $S_{1}(\mathscr{O}, \mathscr{O})$ fails to win the game.

Corollary 8.4.6. $S_{1}^{\mathrm{s}}(\mathscr{O}, \mathscr{O}) \bmod 1 \Longleftrightarrow S_{1}(\mathscr{O}, \mathscr{O}) \bmod 1 \Longleftrightarrow S_{\text {bnd }}(\mathscr{O}, \mathscr{O})$.

Corollary 8.4.7. Let $X$ be a regular space. Then $\mathrm{S}_{\mathrm{bnd}}(\mathscr{O}, \mathscr{O})$ holds if, and only if, there is a compact set $K \subset X$ such that, for every open set $V \supset K, \mathrm{~S}_{1}(\mathscr{O}, \mathscr{O})$ holds on $X \backslash V$.

With the help of Corollary 8.4.7 we can even characterize some metrizable spaces. We just need to consider Theorem 5.2.8 from Fremlin and Miller.

Corollary 8.4.8. Let $(X, \tau)$ be a metrizable space. Then $\mathrm{S}_{\mathrm{bnd}}(\mathscr{O}, \mathscr{O})$ holds if, and only if, there is a compact set $K \subset X$ and a set $N \subset X$ that is strong measure zero with respect to every metric that gives topology $\tau$ such that $X=K \cup N$.

\subsection{The dual game}

Our goal here is to find a duality similar to 4.1.2 for $\mathrm{G}_{\mathrm{bnd}}(\mathscr{O}, \mathscr{O})$, that is, to find a variation of the point-open game that is dual to $\mathrm{G}_{\mathrm{bnd}}(\mathscr{O}, \mathscr{O})$. So, consider the following.

Definition 8.5.1. Given a space $X$, we denote by $\mathrm{G}(X)$ the following game played between AlICE and BoB: in the first inning, AlICE chooses a compact set $K_{0}$ and BoB responds with $V_{0} \supset K_{0}$ open. Then in each inning $n>0$ AlicE chooses $x_{n} \in X$ and BoB responds with an open neighborhood $V_{n}$ of $x_{n}$. ALICE wins the game if $\bigcup_{n \in \omega} V_{n}=X$ and BoB wins otherwise. 
In this case, our duality naturally rises as a simple translation of Theorems 8.3.16, 8.4.1 and Corollary 8.4.7:

Theorem 8.5.2. For every topological space:

(a) If $\operatorname{ALICE} \uparrow \mathrm{G}_{\mathrm{bnd}}(\mathscr{O}, \mathscr{O})$, then $\operatorname{BoB} \uparrow \mathrm{G}(X)$;

(b) If ALICE $\uparrow \mathrm{G}(X)$, then $\mathrm{BOB} \uparrow \mathrm{G}_{\mathrm{bnd}}(\mathscr{O}, \mathscr{O})$.

Moreover, if $X$ is a regular space:

(c) If $\mathrm{BoB} \uparrow \mathrm{G}_{\mathrm{bnd}}(\mathscr{O}, \mathscr{O})$, then $\operatorname{AlicE} \uparrow \mathrm{G}(X)$;

(d) If $\mathrm{BOB} \uparrow \mathrm{G}(X)$, then $\operatorname{AliCE} \uparrow \mathrm{G}_{\mathrm{bnd}}(\mathscr{O}, \mathscr{O})$.

Proof. Assertions (a) and (b) can be easily checked. Assertion (c) follows directly from Theorems 8.3.16 and 4.1.2.

Now, suppose $\mathrm{B}$ OB $\uparrow \mathrm{G}(X)$. Then for every $K \subset X$ compact there is a $V \supset K$ open such that Bов has a winning strategy in the point open game on $X \backslash V$. By 4.1.2, this implies that for

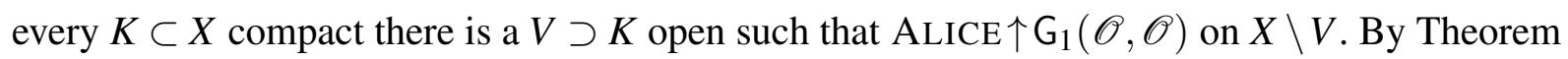
4.1.32, this means that for every $K \subset X$ compact there is a $V \supset K$ open such that $\mathrm{S}_{1}(\mathscr{O}, \mathscr{O})$ fails over $X \backslash V$. Since $X$ is regular, by Corollary 8.4.7, this is equivalent to $\mathrm{S}_{\text {bnd }}(\mathscr{O}, \mathscr{O})$ failing on $X$, which, by Theorem 8.4.1, is equivalent to $\operatorname{ALICE} \uparrow G_{\text {bnd }}(\mathscr{O}, \mathscr{O})$, as we wanted to prove.

We then end this section showing that the assumption of $X$ being a regular space is actually required in the proof of (c) and (d) in Theorem 8.5.2:

Proposition 8.5.3. There is a Hausdorff and non-regular space $X$ such that $\mathrm{BoB} \uparrow \mathrm{G}_{\mathrm{bnd}}(\mathscr{O}, \mathscr{O})$, but $\mathrm{BoB} \uparrow \mathrm{G}(X)$.

Proof. Let $(X, \tau)$ be a Hausdorff space such that $\mathrm{BOB} \uparrow \mathrm{G}_{\mathrm{bnd}}(\mathscr{O}, \mathscr{O})$ and Вов has a winning strategy in the point-open game (for instance, $2^{\omega}$ ) and consider a new topology $\rho$ over $X$ that additionally makes every countable set closed.

Clearly, Вов still has a winning strategy in the point-open game (or, equivalently, the finite-open game) over the new topological space. Moreover, it is easy to see that, in the new topology, $K \subset X$ is compact if, and only if, $K$ is finite. So it follows that $\mathrm{BoB} \uparrow \mathrm{G}((X, \rho))$.

However, BОВ still has a winning strategy in $\mathrm{G}_{\mathrm{bnd}}(\mathscr{O}, \mathscr{O})$ over the new topological space $(X, \rho)$. To see that, we first let $\left\{A_{k}: k \in \omega\right\}$ be a partition of the odd numbers in $\omega$ made by infinite subsets such that $\min A_{i}<\min A_{j}$ when $i<j$ and let $\sigma$ be a winning strategy for Bов in $\mathrm{G}_{1}(\mathscr{O}, \mathscr{O}) \bmod 1$ over the original topological space (that exists, because $(X, \rho)$ remains Hausdorff and by Theorem 8.3.13). In the new space, we may assume that ALICE chooses only 
covers with open sets of the form $U \backslash C$, with $U \in \tau$ and $C$ countable. Given $\mathscr{U}$ open cover of $(X, \rho)$ with said form we fix, for each $U \in \mathscr{U}, U^{\prime}$ as the open set from the original topology such that $U=U^{\prime} \backslash C$ for some $C$ countable. Then we let, for each open cover $\mathscr{U}$ of $(X, \rho)$ with said form,

$$
\mathscr{U}^{\prime}=\left\{U^{\prime} \in \tau: U \in \mathscr{U}\right\} .
$$

Now we define a strategy $\tilde{\sigma}$ as it follows:

- In the first inning $(n=0)$, if ALICE chooses $\mathscr{U}_{0}$, let

$$
\tilde{\sigma}\left(\left\langle\mathscr{U}_{0}\right\rangle\right)=\left\{U \in \mathscr{U}_{0}: U^{\prime} \in \sigma\left(\left\langle\mathscr{U}_{0}^{\prime}\right\rangle\right)\right\}
$$

Note that $\bigcup \sigma\left(\left\langle\mathscr{U}_{0}^{\prime}\right\rangle\right) \backslash \bigcup \tilde{\sigma}\left(\left\langle\mathscr{U}_{0}\right\rangle\right)$ is countable. Then we let $\tilde{\sigma}$ cover these points in the odd innings of the set $A_{0}$.

- If in the next even inning $(n=2)$, ALICE chooses $\mathscr{U}_{2}$, let

$$
\tilde{\sigma}\left(\left\langle\mathscr{U}_{0}, \mathscr{U}_{1}, \mathscr{U}_{2}\right\rangle\right)=\left\{U \in \mathscr{U}_{2}: U^{\prime} \in \sigma\left(\left\langle\mathscr{U}_{0}^{\prime}, \mathscr{U}_{2}^{\prime}\right\rangle\right)\right\}
$$

Note that $\bigcup \sigma\left(\left\langle\mathscr{U}_{0}^{\prime}, \mathscr{U}_{2}^{\prime}\right\rangle\right) \backslash \bigcup \tilde{\sigma}\left(\left\langle\mathscr{U}_{0}, \mathscr{U}_{1}, \mathscr{U}_{2}\right\rangle\right)$ is countable. Then we let $\tilde{\sigma}$ cover these points in the odd innings of the set $A_{1}$.

- If in the next even inning $(n=4)$, ALICE chooses $\mathscr{U}_{4}$, let

$$
\tilde{\sigma}\left(\left\langle\mathscr{U}_{0}, \mathscr{U}_{1}, \mathscr{U}_{2}, \mathscr{U}_{3}, \mathscr{U}_{4}\right\rangle\right)=\left\{U \in \mathscr{U}_{4}: U^{\prime} \in \sigma\left(\left\langle\mathscr{U}_{0}^{\prime}, \mathscr{U}_{2}^{\prime}, \mathscr{U}_{4}^{\prime}\right\rangle\right)\right\}
$$

Note that $\bigcup \sigma\left(\left\langle\mathscr{U}_{0}^{\prime}, \mathscr{U}_{2}^{\prime}, \mathscr{U}_{4}^{\prime}\right\rangle\right) \backslash \bigcup \tilde{\sigma}\left(\left\langle\mathscr{U}_{0}, \mathscr{U}_{1}, \mathscr{U}_{2}, \mathscr{U}_{3}, \mathscr{U}_{4}\right\rangle\right)$ is countable. Then we let $\tilde{\sigma}$ cover these points in the odd innings of the set $A_{2}$.

- And so on.

Clearly, $\tilde{\sigma}$ is a winning strategy in $\mathrm{G}_{\mathrm{bnd}}(\mathscr{O}, \mathscr{O})$ over $(X, \rho)$, and the proof is complete.

Corollary 8.5.4. There is a Hausdorff non-regular space $X$ such that $\mathrm{S}_{\mathrm{bnd}}(\mathscr{O}, \mathscr{O})$ holds, but for every compact $K \subset X$ there is an open set $V \supset K$ such that $S_{1}(\mathscr{O}, \mathscr{O})$ fails over $X \backslash V$.

\subsection{Conclusion}

The results obtained in this paper can be summarized in the following diagrams (Figure 5 is dedicated to the tightness case and Figure 6 is dedicated to the covering case). Arrows represent implications. The number immediately next to an arrow tells us where the proof of such implication is (if it is not obvious) and the number between parenthesis immediately next to it points out to the counterexample of its converse implication. Indications such as "Regular" or " $T_{2}$ " next to an arrow tell us that this assumption was required in the specified proof and the 
number between parenthesis next to this indication points out to the counterexample showing that without said assumption the implication would fail. For simplicity's sake, we will denote "AliCE" by "A" and "BoB" by "B".

With all of that in mind, we quote here some results that show counterexamples to some of the implications in the diagram.

Proposition 8.6.1 ([Gruenhage 2006], Example 2.11; [7], Example 3.10). There is a countable space with only one non-isolated point $p$ on which $\operatorname{ALICE}_{\chi} \mathrm{G}_{1}\left(\Omega_{p}, \Omega_{p}\right)$ and $\mathrm{BOB} \nmid \mathrm{G}_{\mathrm{fin}}\left(\Omega_{p}, \Omega_{p}\right)$.

Proposition 8.6.2 ([Scheepers 1997], pp. 250-251; [7], Example 2.4). There exists a countable space $X$ with only one non-isolated point $p$ on which $\mathrm{S}_{1}\left(\Omega_{p}, \Omega_{p}\right)$ holds (hence, $\mathrm{S}_{\text {fin }}\left(\Omega_{p}, \Omega_{p}\right)$ holds) and $\mathrm{ALICE} \uparrow \mathrm{G}_{\mathrm{fin}}\left(\Omega_{p}, \Omega_{p}\right)$.

Theorem 8.6.3 ([Barman and Dow 2011], Theorem 3.6). If $X$ is $\sigma$-compact and metrizable, then $\mathrm{BoB} \uparrow \mathrm{G}_{\mathrm{fin}}\left(\Omega_{0}, \Omega_{0}\right)$ on $C_{p}(X)$.

Theorem 8.6.4 ([Sakai 1988], Theorem 1). For every space $X, \mathrm{~S}_{1}\left(\Omega_{0}, \Omega_{0}\right)$ holds over $C_{p}(X)$ if, and only if, $\mathrm{S}_{1}(\mathscr{O}, \mathscr{O})$ holds on each finite product of $X$.

Corollary 8.6.5. On $C_{p}(\mathbb{R})$ :

(a) $\mathrm{BoB} \uparrow \mathrm{G}_{\text {fin }}\left(\Omega_{0}, \Omega_{0}\right)$;

(b) $\mathrm{S}_{1}\left(\Omega_{0}, \Omega_{0}\right)$ fails.

Proposition 8.6.6 ([Telgársky 1983], Section 7; [Aurichi and Dias 2013], Example 3.5). There is a space on which $\mathrm{S}_{1}(\mathscr{O}, \mathscr{O})$ holds (hence, $\operatorname{ALICE}_{\gamma} \mathrm{G}_{1}(\mathscr{O}, \mathscr{O})$ ), but $\operatorname{BoB} \nmid \mathrm{G}_{\text {fin }}(\mathscr{O}, \mathscr{O})$. 


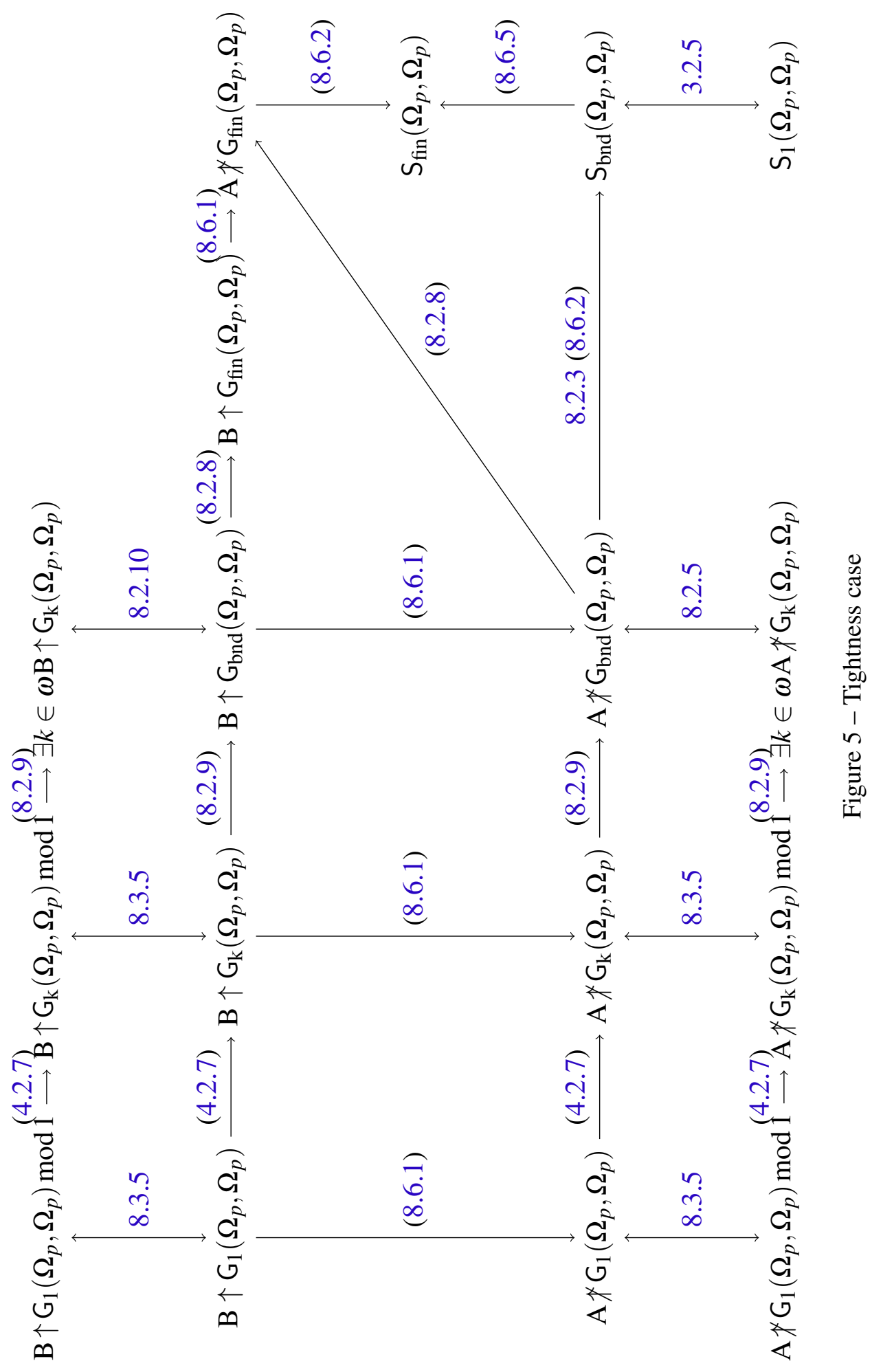




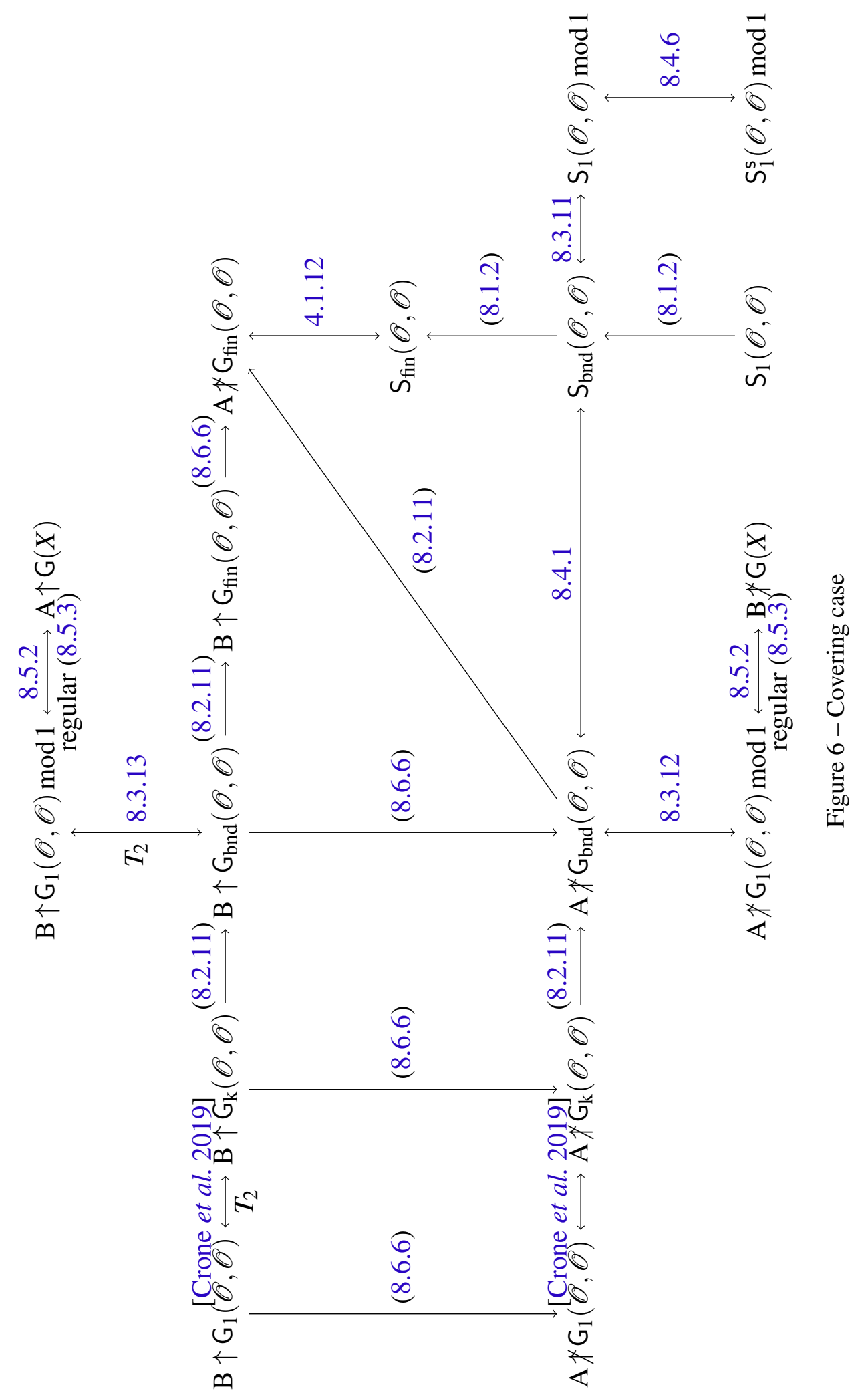


In the proof of Theorem 8.3.13 we used the main result of [Crone et al. 2019], which is why we required $X$ to be Hausdorff. So, just like it was done in [Crone et al. 2019], it is only natural to end here with the question:

Problem 8.6.7. Is there a non-Hausdorff space $X$ such that $\mathrm{B}$ ов $\uparrow \mathrm{G}_{\mathrm{bnd}}(\mathscr{O}, \mathscr{O})$, but $\mathrm{B}$ oв $\nmid \mathrm{G}_{1}(\mathscr{O}, \mathscr{O}) \bmod 1$ ?

In fact, it is easy to see that Problem 8.6.7 is actually equivalent to the problem presented in [Crone et al. 2019]:

Problem 8.6.8. Is there a non-Hausdorff space $X$ such that $\mathrm{BOB} \uparrow \mathrm{G}_{\mathrm{k}}(\mathscr{O}, \mathscr{O})$ for some $k \in \mathbb{N}$, but Вов $\Varangle \mathrm{G}_{1}(\mathscr{O}, \mathscr{O})$ ? 

ALSTER, K. On the class of all spaces of weight not greater than $\omega_{1}$ whose Cartesian product with every Lindelöf space is Lindelöf. Fund. Math., v. 129, n. 2, p. 133-140, 1988. ISSN 0016-2736. Citation on page 106.

. The product of a Lindelöf space with the space of irrationals under Martin's axiom. Proc. Amer. Math. Soc., v. 110, n. 2, p. 543-547, 1990. ISSN 00029939. Citation on page 106.

ARHANGEL'SKII, A. V. The frequency spectrum of a topological space and the classification of spaces. Dokl. Akad. Nauk SSSR, v. 206, n. 2, p. 265-268, 1972. Citation on page 114.

. Hurewicz spaces, analytic sets and fan tightness of function spaces. Dokl. Akad. Nauk SSSR, v. 287, n. 3, p. 525-528, 1986. Citation on page 119.

AURICHI, L. F. $D$-spaces, topological games and selection principles. Topol. Proc., v. 36, p. 107-122, 2010. Citation on page 110.

AURICHI, L. F; BELLA, A. Topological games and productively countably tight spaces. Topol. Appl., Elsevier B.V., v. 171, p. 7-14, 2014. ISSN 17264642. Citation on page 115.

AURICHI, L. F.; BELLA, A.; DIAS, R. R. Tightness games with bounded finite selections. Isr. J. Math., v. 224, n. 1, p. 133-158, 2018. Citations on pages 91, 135, 137, and 154.

AURICHI, L. F.; DIAS, R. R. Topological games and Alster spaces. Canad. Math. Bull, v. 57, n. 4, p. 683-696, 2013. Citation on page 154.

. A minicourse on topological games. Topol. Appl., Elsevier B.V., v. 258, p. 305-335, 2019. ISSN 01668641. Citation on page 15.

AURICHI, L. F; DUZI, M. Topological games of bounded selections. arXiv:1901.09371, 2019. Citations on pages 17 and 133.

BARMAN, D.; DOW, A. Selective Separability and SS+. Topol. Proc., v. 37, p. 181-204, 2011. ISSN 01464124. Citation on page 154.

BUKOVSKÝ, L. The Structure of the Real Line. Phd Thesis (PhD Thesis) - Instytut Matematyczny Polskiej Akademii Nauk, 2010. Citation on page 55.

CIESIELSKI, K. Set Theory for the Working Mathematician. 1. ed. Cambridge: Cambridge University Press, 1997. 103 p. Citation on page 46.

COHEN, P. Products of Baire Spaces. Proc. Amer. Math. Soc., v. 55, n. 1, p. 119-124, 1976. Citation on page 99.

CRONE, L.; FISHMAN, L.; HIERS, N.; JACKSON, S. Equivalence of the Rothberger, $k$ Rothberger, and restricted Menger Games. Topol. Appl., Elsevier B.V., v. 258, p. 172-176, 2019. ISSN 01668641. Citations on pages 84, 156, and 157. 
ENGELKING, R. General Topology. 2. ed. Berlin: Heldermann Verlag, 1989. Citation on page 21 .

GALVIN, F. Indeterminacy of point-open games. Bull. l'Académie Pol. des Sci., v. 26, p. 445-449, 1978. Citations on pages 70, 71, and 134.

GALVIN, F.; TELGÁRSKY, R. Stationary strategies in topological games. Topol. Appl., v. 22, p. 51-69, 1986. Citations on pages 17, 37, 39, and 129.

GARCÍA-FERREIRA, S.; TAMARIZ-MASCARÚA, A. Some generalizations of rapid ultrafilters in topology and Id-fan tightness. Tsukuba J. Math, v. 19, n. 1, p. 173-185, 1995. ISSN 0387-4982. Citations on pages 51, 135, and 137.

GERLITS, J.; NAGY, Z. Some properties of $C(X)$, I. Topol. Appl., v. 14, n. 2, p. 151-161, 1982. ISSN 01668641 . Citation on page 61.

GRUENHAGE, G. Infinite games and generalizations of first-countable spaces. Gen. Topol. its Appl., v. 6, n. 3, p. 339-352, 1976. ISSN 0016660X. Citations on pages 36, 93, 94, and 117.

The story of a topological game. Rocky Mt. J. Math., v. 36, n. 6, p. 1885-1914, 2006. ISSN 00167037 . Citations on pages 95 and 154.

HUREWICZ, W. Über eine Verallgemeinerung des Borelschen Theorems. Math. Z., v. 24, p. 401-421, 1926. Citation on page 74.

JUST, W.; MILLER, A. W.; SCHEEPERS, M.; SZEPTYCKI, P. J. The combinatorics of open covers II. Topol. Appl., v. 73, n. 3, p. 241-266, 1996. Citation on page 63.

KALMÁR, L. Zur Theorie der abstrakten Spiele. Acta Sci. Math., v. 4, p. 65-85, 1928. Citation on page 33 .

KHOMSKII, Y. Infinite Games. 2010. Available: <https://www.math.uni-hamburg.de/home/ khomskii/infinitegames2010/>. Citation on page 33.

MATVEEV, M. Rothberger property and purely atomic measures Rothberger spaces. Quest. Answers Gen. Topol., v. 28, n. 2, p. 157-160, 2010. Citations on pages 103 and 104.

MICHAEL, E. Paracompactness and the Lindelöf property in finite and countable Cartesian products. Compos. Math., v. 23, n. 2, p. 199-214, 1971. Citation on page 106.

MILLER, A. W.; FREMLIN, D. H. On some properties of Hurewicz, Menger and Rothberger. Fund. Math., v. 129, p. 17-33, 1988. Citation on page 101.

OXTOBY, J. C. The Banach-Mazur game and Banach category theorem. In: Contrib. to Theory Games. Vol. III. [S.1.]: Princeton University Press, 1957. p. 159-163. Citation on page 97.

PAWLIKOWSKI, J. Undetermined sets of point-open games. Fund. Math., v. 144, n. 3, p. 279-285, 1994. ISSN 0016-2736. Citation on page 82.

PENG, L. X. On some sufficiencies of $D$-spaces. J. Beijing Inst. Technol., v. 16, p. 229-233, 1996. Citation on page 111.

On finite unions of certain D-spaces. Topol. Appl., v. 155, n. 6, p. 522-526, 2008. ISSN

01668641. Citation on page 112. 
REPOVs, D.; ZDOMSKYY, L. On the Menger covering property and $D$-spaces. Proc. Amer. Math. Soc., v. 140, n. 3, p. 1069-1074, 2012. Citation on page 107.

ROTHBERGER, F. Eine Verschärfung der Eigenschaft C. Fund. Math., v. 30, p. 50-55, 1938. ISSN 0016-2736. Citation on page 54.

SAKAI, M. Property $C^{\prime \prime}$ and function spaces. Proc. Amer. Math. Soc., v. 104, n. 3, p. 917-919, 1988. ISSN 0006-4971. Citations on pages 65, 118, 121, and 154.

SCHEEPERS, M. Combinatorics of open covers I: Ramsey theory. Topol. Appl., v. 69, n. 1, p. 31-62, 1996. ISSN 01668641. Citations on pages 60 and 87.

. Combinatorics of open covers (III): Games, $C_{p}(X)$. Fund. Math., v. 152, n. 3, p. 231-254, 1997. ISSN 00162736. Citations on pages 90, 118, 119, 121, and 154.

. Remarks on countable tightness. Topol. Appl., Elsevier B.V., v. 161, n. 1, p. 407-432, 2014. ISSN 01668641. Citations on pages 120 and 121.

SZEWCZAK, P.; TSABAN, B. Conceptual proofs of the Menger and Rothberger games. arXiv:1904.02736, p. 1-7, 2019. Citations on pages 74, 82, 134, and 147.

TELGÁRSKY, R. On sieve-complete and compact-like spaces. Topol. Appl., v. 16, n. 1, p. 61-68, 1983. ISSN 01668641. Citations on pages 108 and 109.

Spaces defined by topological games. Fund. Math., v. 116, n. 3, p. 189-207, 1983. Citations on pages $71,79,80,88,114$, and 154 .

On games of Topsøe. Math. Scand., v. 54, n. 1984, p. 170-176, 1984. ISSN 0025-5521. Citation on page 76.

Topological games: on the 50th anniversary of the Banach-Mazur game. J. Math., v. 17, n. 2, p. 227-276, 1987. Citation on page 15.

ZDOMSKYY, L. A semifilter approach to selection principles. Comment. Math. Univ. Carolin., v. 46, n. 3, p. 525-539, 2005. Citation on page 107.

ZERMELO, E. Über eine Anwendung der Mengenlehre auf die Theorie des Schachspiels. E.W. Hobson, A.E.H. Love (Eds.), Proc. Fifth Int. Congr. Math., v. 2, p. 501-504, 1913. Citation on page 33 . 

$C(X), 118$

$C_{p}(X), 118$

D

space, 109

$T_{1}$

space, 22

$T_{2}$

space, 22

$T_{3}$

space, 23

$T_{4}$

space, 23

$T_{3 \frac{1}{2}}$

space, 23

$\Gamma, 55$

$\boldsymbol{k}$-Rothberger

game, 68

$\mathbb{N}, 21$

$\mathscr{K}_{\delta}, 58$

$\mathrm{D}, 52$

$\operatorname{BM}(X), 43$

$\mathrm{G}_{1}(\mathscr{A}, \mathscr{B}), 67$

$\mathrm{G}_{\text {bnd }}(\mathscr{A}, \mathscr{B}), 136$

$\mathrm{G}_{\mathrm{f}}(\mathscr{A}, \mathscr{B}), 136$

$\mathrm{G}_{\mathrm{k}}(\mathscr{A}, \mathscr{B}), 68$

$\mathrm{S}_{1}(\mathscr{A}, \mathscr{B}), 50$

$\mathrm{S}_{\text {bnd }}(\mathscr{A}, \mathscr{B}), 134$

$\mathrm{S}_{\text {fin }}(\mathscr{A}, \mathscr{B}), 50$

$\mathrm{S}_{\mathrm{k}}(\mathscr{A}, \mathscr{B}), 50$

$\mathrm{U}_{\text {fin }}(\mathscr{A}, \mathscr{B}), 55$

$\sigma$-compact, 23

Alice, 28, 29

Bов, 28, 29
${ }^{<\omega} A, 21$

$p \perp q, 21$

$\Omega, 60$

$\Omega_{x}, 51$

$\mathscr{L}, 49$

$\mathscr{O}, 49$

$\mathscr{O}_{\delta}, 58$

$\mathfrak{c}, 21$

$\left(\begin{array}{c}\mathscr{A} \\ \mathscr{B}\end{array}\right), 49$

Čech

complete, 57

$K$-cover, 58

$\gamma$-cover, 55

$\pi$-basis, 95

$\omega$-cover, 60

Alster

cover, 58

game, 87

space, 58

atom, 102

Baire

productively, 99

space, 97

Banach-Mazur

game, 43

base

filter, 22

Bernstein

set, 46

branching

finitely, 22

clusters at, 25 
clusters in, 25

compact- $G_{\delta}$

game, 88

compact-like, 76

compact-open

game, 75

compact-valued, 106

compactification, 25

compatible, 21, 31

complete

Čech, 57

sieve, 107

completely

regular space, 23

convergence

game, neighborhood-point, 94

countable

fan-tightness, 51

strong fan-tightness, 51

type, 59

countably

tight, 114

tight, productively, 117

cover

Alster, 58

DC-like, 111

DC-open

game, 111

dense

nowhere, 23

determined, 33

discrete

family, 24

space, 24

dual

games, 70

dual over, 69

equivalent games, 34

equivalent over, 34

family

discrete, 24

fan-tightness

countable, 51

countable strong, 51

filter

base, 22

finite

game, 28

locally, 24

finite-open

game, 30

game, strict, 36

finitely

branching, 22

game

$\boldsymbol{k}$-Rothberger, 68

Alster, 87

Banach-Mazur, 43

compact- $G_{\delta}, 88$

compact-open, 75

DC-open, 111

finite, 28

finite-open, 30

Hurewicz, 87

increasing point-open, 37

infinite, 29

Menger, 68

neighborhood-point, 92

neighborhood-point convergence, 94

open-point, 95

point-open, 30

positional, 39

Rothberger, 67

sieve, 107

strict finite-open, 36 
games

dual, 70

equivalent, 34

tightness, 90

Hausdorff

space, 22

Hurewicz

game, 87

space, 55

incompatible, 21

increasing point-open

game, 37

infinite

game, 29

inning, 28, 29

Lindelöf, 23

Lindelöf

productively , 105

local character, 24

locally

finite, 24

Luzin

set, 23

map

set-valued, 106

measure

purely atomic, 102

regular, 102

Menger

game, 68

space, 50

Michael

space, 106

moment, 28, 29

neighborhood-point

convergence game, 94

game, 92 normal

space, 23

nowhere

dense, 23

o.n.a., 109

open neighborhood assignment, 109

open-point

game, 95

payoff, 28, 29

point-open

game, 30

positional

game, 39

strategy, 39

productively

Baire, 99

countably tight, 117

productively

Lindelöf, 105

purely atomic

measure, 102

regular

measure, 102

space, 23

space, completely, 23

root, 22

Rothberger

game, 67

space, 50

run, 28,29

set

Bernstein, 46

Luzin, 23

set-valued

map, 106

sieve

complete, 107 
game, 107

sieve-complete, 107

space, 22

space

$D, 109$

$T_{1}, 22$

$T_{2}, 22$

$T_{3}, 23$

$T_{4}, 23$

$T_{3 \frac{1}{2}}, 23$

Alster, 58

Baire, 97

completely regular, 23

discrete, 24

Hausdorff, 22

Hurewicz, 55

Menger, 50

Michael, 106

normal, 23

regular, 23

Rothberger, 50

strategy, 30, 31 strategy

positional, 39

winning, 31

strict

finite-open game, 36

strong

fan-tightness, countable, 51

strong measure zero, 100

successor, 22

tight

countably, 114

productively countably, 117

tightness

games, 90

tree, 22

type

countable, 59

undetermined, 33

upper semicontinuos, 106

winning

strategy, 31 


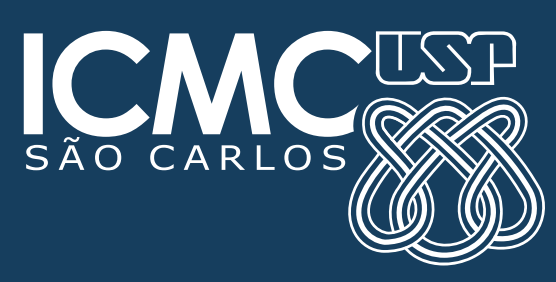

